Elke Rajal, trafo.K, Oliver Marchart, Nora Landkammer, Carina Maier (Hg.)

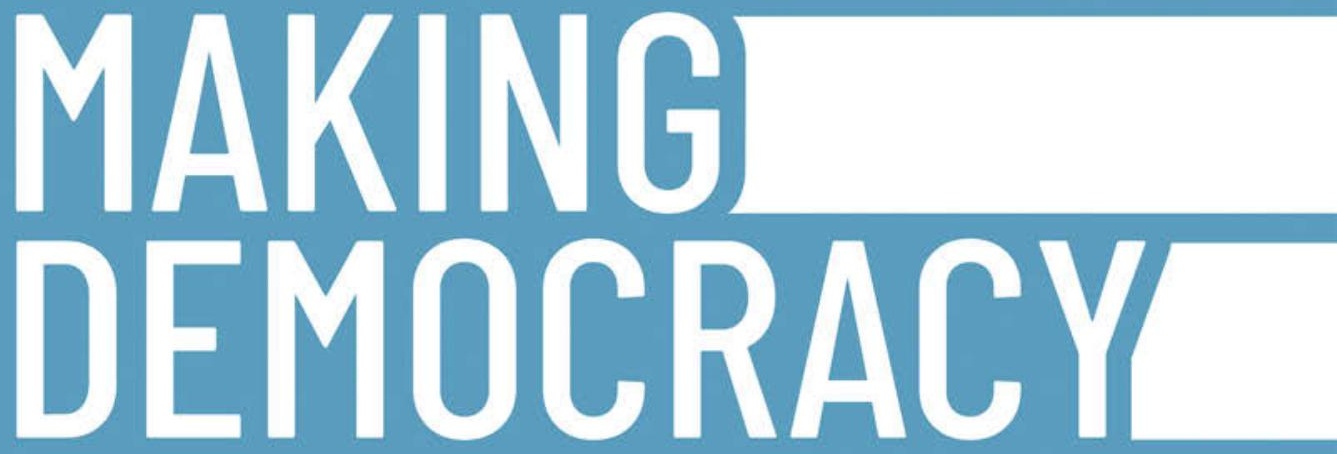

\title{
Aushandlungen von Freiheit, Gleichheit und Solidarität im Alltag
}

[transcript] EditionPolitik 
Elke Rajal, trafo.K, Oliver Marchart, Nora Landkammer, Carina Maier (Hg.) Making Democracy -

Aushandlungen von Freiheit, Gleichheit und Solidarität im Alltag 
Die freie Verfügbarkeit der E-Book-Ausgabe dieser Publikation wurde ermöglicht durch den Fachinformationsdienst Politikwissenschaft POLLUX

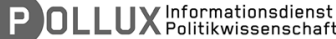

und ein Netzwerk wissenschaftlicher Bibliotheken zur Förderung von Open Access in den Sozial- und Geisteswissenschaften (transcript, Politikwissenschaft 2020)

Die Publikation beachtet die Qualitätsstandards für die Open-Access-Publikation von Büchern (Nationaler Open-Access-Kontaktpunkt et al. 2018), Phase 1

https://oa2020-de.org/blog/2018/o7/31/empfehlungen_qualitätsstandards_oabücher/

Universitätsbibliothek Bayreuth |

Universitätsbibliothek der Humboldt-

Universität zu Berlin | Staatsbibliothek

zu Berlin | Universitätsbibliothek FU

Berlin | Universitätsbibliothek Bielefeld

(University of Bielefeld) | Universitäts-

bibliothek der Ruhr-Universität Bochum

| Universitäts- und Landesbibliothek |

Sächsische Landesbibliothek - Staats- und

Universitätsbibliothek Dresden | Universi-

tätsbibliothek Duisburg-Essen | Univer-

sitäts- u. Landesbibliothek Düsseldorf |

Universitätsbibliothek Erlangen-Nürnberg

| Universitätsbibliothek Johann Christian

Senckenberg | Universitätsbibliothek

Gießen | Niedersächsische Staats- und Universitätsbibliothek Göttingen | Universitäts-

bibliothek Graz | Universitätsbibliothek der FernUniversität in Hagen | Martin-LutherUniversität Halle-Wittenberg | Staats- und Universitätsbibliothek Carl von Ossietzky,

Hamburg | Technische Informations-

bibliothek Hannover | Gottfried Wilhelm

Leibniz Bibliothek - Niedersächsische
Landesbibliothek | Universitätsbibliothek

Kassel | Universitäts- und Stadtbibliothek

Köln | Universität Konstanz, Kommuni-

kations-, Informations-, Medienzentrum

| Universitätsbibliothek Koblenz-Landau | Universitätsbibliothek Leipzig | Zentral- u. Hochschulbibliothek Luzern | Universitätsbibliothek Mainz | Universitätsbibliothek Marburg | Ludwig-Maximilians-Universität München Universitätsbibliothek | Max Planck Digital Library | Universitäts- und Landesbibliothek Münster | Universitätsbibliothek Oldenburg | Universitätsbibliothek Osnabrück| Universitätsbibliothek Passau | Universitätsbibliothek Potsdam | Universitätsbibliothek Siegen | Universitätsbibliothek Vechta | Universitätsbibliothek der Bauhaus-Universität Weimar | Universitätsbibliothek Wien | Universitätsbibliothek Wuppertal | Universitätsbibliothek Würzburg | Zentralbibliothek Zürich | Bundesministerium der Verteidigung - Bibliothek | Landesbibliothek Oldenburg 
Elke Rajal, trafo.K, Oliver Marchart,

Nora Landkammer, Carina Maier (Hg.)

Making Democracy -

Aushandlungen von Freiheit, Gleichheit und Solidarität im Alltag

[transcript] 


\section{Bibliografische Information der Deutschen Nationalbibliothek}

Die Deutsche Nationalbibliothek verzeichnet diese Publikation in der Deutschen Nationalbibliografie; detaillierte bibliografische Daten sind im Internet über http://dnb.d-nb.de abrufbar.

\section{(9) $(1) \Theta \Theta$}

Dieses Werk ist lizenziert unter der Creative Commons Attribution-NonCommercialNoDerivs 4.० Lizenz (BY-NC-ND). Diese Lizenz erlaubt die private Nutzung, gestattet aber keine Bearbeitung und keine kommerzielle Nutzung. Weitere Informationen finden Sie unter https://creativecommons.org/licenses/by-nc-nd/4.o/deed.de Um Genehmigungen für Adaptionen, Übersetzungen, Derivate oder Wiederverwendung zu kommerziellen Zwecken einzuholen, wenden Sie sich bitte an rights@ transcript-verlag.de

Die Bedingungen der Creative-Commons-Lizenz gelten nur für Originalmaterial. Die Wiederverwendung von Material aus anderen Quellen (gekennzeichnet mit Quellenangabe) wie z.B. Schaubilder, Abbildungen, Fotos und Textauszüge erfordert ggf. weitere Nutzungsgenehmigungen durch den jeweiligen Rechteinhaber.

\section{(C) 2020 transcript Verlag, Bielefeld}

Umschlaggestaltung: Maria Arndt, Bielefeld

Redaktion: Nora Landkammer, Carina Maier, Elke Rajal

Visualisierung: Renate Höllwart, Elke Smodics

Lektorat: Jannik Eder, Wien

Satz: Michael Rauscher, Bielefeld

Druck: Majuskel Medienproduktion GmbH, Wetzlar

Print-ISBN 978-3-8376-5016-7

PDF-ISBN 978-3-8394-5016-1

https://doi.org/10.14361/9783839450161

Gedruckt auf alterungsbeständigem Papier mit chlorfrei gebleichtem Zellstoff. Besuchen Sie uns im Internet: https://www.transcript-verlag.de Unsere aktuelle Vorschau finden Sie unter www.transcript-verlag.de/vorschaudownload 
Dieses Buch entstand im Rahmen des Projekts Making Democracy. Aushandlungen von Freiheit, Gleichheit und Solidarität unter Jugendlichen, durchgeführt im Rahmen des Förderprogramms Sparkling Science, gefördert vom Bundesministerium für Wissenschaft, Forschung und Wirtschaft.

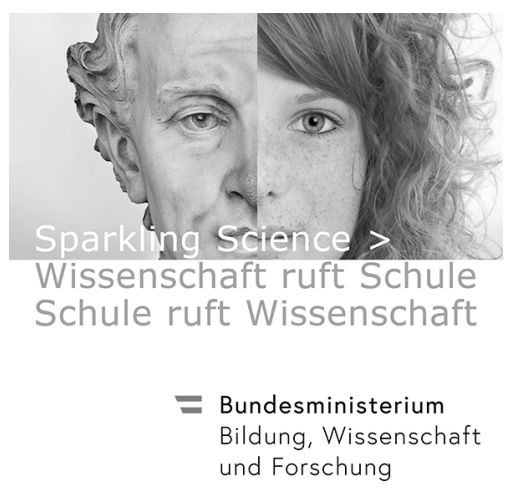

Projektteam:

Oliver Marchart, Elke Rajal, Ines Garnitschnig, Nora Landkammer, Carina Maier (Institut für Politikwissenschaft der Universität Wien)

Renate Höllwart, Elke Smodics (trafo.K - Büro für Kunstvermittlung und kritische Wissensproduktion)

Aslı Kışlal, Anna Schober und Schauspielstudierende (diverCITYLAB - PERFORMANCE- und THEATERlabor)

Ka Schmitz (Künstlerin, Comic-Zeichnerin)

Schüler*innen der 3A und 3B (Schuljahr 2017/18), der 7B (Schuljahr 2018/19) sowie Lehrer`innen, insb. Beate Wallner und Stefanie Schermann (WMS/BG/ORG antonkriegergasse)

Nora Sternfeld (wissenschaftliche Begleitung)

Sandra Kosel (Video- und Fotodokumentation) 



\section{Inhalt}

Danksagungen 9

\section{Einleitung}

Demokratie aushandeln

Elke Rajal, Carina Maier, Nora Landkammer 11

Demokratie im Alltag

Anmerkungen zum politik-theoretischen Untersuchungsdesign von Making Democracy

Oliver Marchart 23

Making Democracy. Aushandlungen von Freiheit, Gleichheit und Solidarität unter Jugendlichen

Erkenntnisse aus dem Prozess

Elke Rajal 33

Das Projekt Making Democracy als Gegenstand demokratischer Forderungen und als Raum für deren Artikulation

Nora Landkammer 49

Idee, Projekt, Vision und dann?

Reflexion zum Thema »Demokratie machen"

Stefanie Schermann, Beate Wallner 63

Wie utopisch ist es, von einem demokratischen Theater und einem demokratischen Lernen zu reden?

Übungen in zwei Systemen

Aslı Kışlal

Taktiken für eine verändernde Praxis

Schnittstellen kritischer Kunstvermittlung und partizipativer Forschung Renate Höllwart, Nora Landkammer, Elke Smodics 
Freiheit in der Schule - oder haben wir die überhaupt?

Eine Selbstbefragung

Forschungsgruppe Freiheit in der Schule, 7B antonkriegergasse

101

Making Democracy in der Schule

Bildstrecke

Konzept: Renate Höllwart, Elke Smodics

Orte der Liebe und der Ausgrenzung

Re-Lektüren einer Forschungsgruppe

Karin Schneider

\section{Making Art, Taking Part!}

DIY-Kulturen und künstlerische Interventionen im Kontext

einer kritischen Vermittlungspraxis

Elke Zobl

Es war einmal ... die Kulturarbeit

Claudia Hummel

Die Verhandlung demokratischer Werte als Kohärenzpraxis

Ein Beispiel für Hegemonie als pädagogisches Verhältnis

Ingo Pohn-Lauggas

Wozu lernen? = Wozu kämpfen?

Pädagogik im globalen postkolonialen Raum

das kollektiv

Demokratie ist radikaler Experimentalismus

Rahel Süß

Eine Demokratie lernen, die es noch nicht gibt

Nora Sternfeld 205

Autor*innen 


\section{Danksagungen}

Wir möchten uns als Projekt- und Redaktionsteam bei allen Kooperationspartner"innen von Making Democracy bedanken: allen voran bei unserer Kollegin Ines Garnitschnig, die das Projekt mitinitiiert hat, im ersten Jahr Teil unseres Teams war und ohne die der Prozess so nicht vonstattengegangen wäre; bei der Künstlerin Ka Schmitz für ihre soziale Bewegtheit im Teilen von Wissen und Expertise in der Durchführung der Comic-Workshops, und bei Gabriele Wappel und Sebastian Radon von schallundrauch agency sowie bei Aslı Kışlal und Anna Schober und den Student*innen von diverCITYLAB für die performative Arbeit mit den Jugendlichen und die gemeinsame Reflexion des Prozesses. Wir bedanken uns bei Beate Wallner und Stefanie Schermann für die unterstützende Zusammenarbeit in allen Belangen des Projektes; ebenso bei ihren am Projekt beteiligten Kolleg*innen Ernst Auer, Josef Eder, Robert Fröhlich, Simone Hofer, Marc Michael Moser, Georg Rakowitz, Olaf Winnecke und Verena Zangerle der antonkriegergasse für ihr Engagement und ihre Ausdauer sowie bei Direktor Michel Fleck, der dem Projekt den notwendigen Rahmen zur Verfügung stellte. Auch möchten wir uns bei der Künstlerin Sandra Kosel bedanken, die mit ihren Fotografien und Videoaufnahmen den Prozess in Bildern festgehalten hat, sowie bei allen weiteren Akteur*innen und Institutionen, wie Lale Rodgarkia-Dara (Radioworkshop) und Katja Stecher (Belvedere 21).

Außerdem bedanken wir uns bei allen Tagungsreferent ${ }^{*}$ innen und -gästen für ihre wertvollen Beiträge, die die Reflexion unseres Projekts und des größeren Zusammenhangs wesentlich bereichert haben, und den Mitarbeiter*innen vom Volkskundemuseum Wien. Danken möchten wir selbstverständlich auch dem Fördergeber (Bundesministerium für Wissenschaft, Forschung und Wirtschaft, im Rahmen des Programms Sparkling Science), der das Projekt Making Democracy und diesen Band erst ermöglicht hat. 
Unser ganz besonderer Dank gilt schließlich den am Projekt beteiligten Schüler*innen (3A, 3B und 7B) für ihre Offenheit, ihr Interesse und Engagement, für die großartigen Ideen und die Diskussionen, die uns inspiriert und herausgefordert haben, und ohne die das Projekt gar nicht hätte stattfinden können.

Wir freuen uns auf weitere Erprobungen von Demokratie mit euch!

\author{
Renate Höllwart \\ Nora Landkammer \\ Carina Maier \\ Oliver Marchart \\ Elke Rajal \\ Elke Smodics
}




\section{Einleitung Demokratie aushandeln}

Elke Rajal, Carina Maier, Nora Landkammer

Freiheit, Gleichheit und Solidarität gehören zu den zentralen Grundwerten der Demokratie. Wie diese Werte allerdings inhaltlich zu bestimmen und umzusetzen sind, ist umkämpft. Während die Rolle politischer Entscheidungsträger*innen und öffentlicher Debatten in dieser Bestimmung demokratischer Grundideen augenfällig ist, bleiben die Arten und Weisen, in denen Menschen in ihrer Alltagspraxis Freiheit, Gleichheit und Solidarität laufend neu verhandeln, häufig ausgeblendet. Wie wird Demokratie in sozialen Beziehungen »gemacht«, beispielsweise in der Verteidigung oder Aufgabe persönlicher Freiheiten, im Zulassen von oder Aufbegehren gegenüber Ungerechtigkeit, in Gesten des Sich-Einsetzens für andere? Wie werden Gleichheit, Freiheit und Solidarität im Alltag verhandelt?

Ausgehend vom Forschungsprojekt Making Democracy und der gleichnamigen Tagung im Mai $2019^{1}$ widmet sich dieser Band den genannten Fragen an einer transdisziplinären Schnittstelle zwischen Politikwissenschaft, Pädagogik, Kunstvermittlung und zeitgenössischer bildender und darstellender Kunst. Das Projekt Making Democracy (durchgeführt im Rahmen des Förderprogramms Sparkling Science, gefördert vom Bundesministerium für Wissenschaft, Forschung und Wirtschaft) untersuchte in den Jahren 2017 bis 2019 gemeinsam mit Kindern und Jugendlichen einer Wiener Schule den alltagskulturellen Aushandlungsraum von Demokratie. Perspektiven der Demokratietheorie wurden dabei mit jenen der kritischen Kunstvermittlung verschränkt, sozialwissenschaftliches Forschen und künstlerische

1 Tagung Making Democracy, 27. und 28. Mai 2019 im Volkskundemuseum Wien, https://poli tikwissenschaft.univie.ac.at/forschung/forschungsprojekte/forschungsprojekt-makingdemocracy/tagung-am-27-und-28-mai-2019/ (letzter Zugriff: 26.8.2019). 
Methoden informierten sich gegenseitig in einem partizipatorischen Forschungssetting.

Der erste Abschnitt des Bandes widmet sich der Auswertung und Reflexion von Making Democracy. Im zweiten Abschnitt wird mit Beiträgen aus Demokratietheorie, Pädagogik und Kunstvermittlung die Frage nach alltäglichen Aushandlungsformen von Demokratie erweitert. Die versammelten Texte geben einen multiperspektivischen Einblick in theoretische Zugänge, Methoden und die Komplexität von Projekten, die demokratische Aushandlungsräume im Bildungs- und Kunstkontext schaffen und verstehen wollen.

Im Folgenden wollen wir das Projekt Making Democracy als Ausgangspunkt skizzieren und einen Einblick in die Beiträge des Buches geben.

\section{Making Democracy - ein partizipatives Forschungsprojekt}

Ziel des Projekts, das vom Institut für Politikwissenschaft der Universität Wien in Kooperation mit Büro trafo.K, diverCITYLAB und der Schule WMS/RG/ORG antonkriegergasse durchgeführt wurde, war es, alltägliche Aushandlungsformen demokratischer Grundwerte durch Jugendliche gemeinsam mit Jugendlichen zu erforschen. Ausgangspunkt für diesen Fokus auf den Alltag waren - neben dem theoretischen Interesse - empirische Untersuchungen zum Zusammenspiel von Politik und Jugend, die häufig feststellen, dass sich Jugendliche weder politisch interessieren noch partizipieren würden und jungen Menschen in hohem Grad »Politikverdrossenheit« attestieren (Soßdorf 2016, 35 f.). Wenn man sich die Ergebnisse genauer ansieht, stellt man fest, dass primär das Vertrauen in die institutionalisierte und verfasste Politik sowie das Interesse an organisierter Parteipolitik schwach ausgeprägt sind (ebd.). Dass Jugendliche nicht »unpolitisch« sind, zeigt etwa die im Herbst 2018 vom Institut SORA ${ }^{2}$ durchgeführte Studie Österreichischer Demokratie Monitor: Hier wird der deutliche Wunsch von in Österreich ansässigen Jugendlichen nach mehr politischer Bildung festgestellt. 52 Prozent der 300 befragten Jugendlichen meinten, in der Schule zu wenig über ihre Rechte als Bürger*innen erfahren zu haben. Gleich viele Jugendliche äußerten, sie hätten in der Schule zu wenig gelernt, wie man politische Debatten führt. Einen Mangel an Bildung über Beteiligungsformen

2 Institute for Social Research and Consulting 
kritisierten 44 Prozent der Jugendlichen. Rund ein Drittel der Befragten der »Generation Z ( (ab dem Geburtsjahr 1997) sagte, sie könnten/konnten in der Schule nie oder nur selten mitbestimmen, 42 Prozent dürfen/durften sich ihrer Aussage nach gelegentlich an Entscheidungen beteiligen und lediglich 23 Prozent oft (SORA/Zandonella 2018). An diesen Zahlen wird sichtbar, dass den Vorwürfen der Politikverdrossenheit die Kritik der Jugendlichen an mangelnder politischer Bildung und fehlenden Mitbestimmungsmöglichkeiten in der Schule diametral gegenübersteht.

Zahlreiche Befunde machen allerdings deutlich, dass Beteiligungs- und Mitgestaltungsmöglichkeiten für das Demokratiebewusstsein von Jugendlichen zentral sind (siehe z. B. Oerter 2016, 81; Palentien 2016, 112). Gegenwärtige Untersuchungen beschränken sich aber meist auf nur wenige Formen der politischen Partizipation (Gürlevik et al. 2016, 8). Erkenntnissen der empirischen Demokratieforschung zufolge muss hinsichtlich der Einstellungen junger Menschen zu Politik zwischen zwei Aspekten unterschieden werden: dem Vertrauen in das politische System und dem allgemeinen Interesse an Politik. Das Vertrauen Jugendlicher in klassische politische Institutionen - wie Parteien, Regierungen, Parlamente - ist ebenso gering wie jenes gegenüber Jugendorganisationen, Gewerkschaften und Interessensverbänden, konstatieren Gürlevik et al. (2016, 6 f.) bezogen auf Deutschland. Institutionelle Politik wird als Sphäre von Unehrlichkeit und Konkurrenzzwängen gefasst: Etwa zwei Drittel der Jugendlichen geben an, ihre Anliegen von Politiker*innen nicht vertreten zu sehen (Filzmaier 2007; Shell Jugendstudie 2015). Einer anderen Studie zufolge hatte 2014 fast die Hälfte der jungen Menschen zwischen 14 und 18 Jahren in Österreich wenig bis kein Vertrauen in das politische System (Großegger/Rohrer 2015). Davon ausgehend auf Politikverdrossenheit unter Jugendlichen zu schließen, wäre jedoch nicht zulässig - vielmehr können wir hier von »Politiker- und Parteienverdrossenheit" sprechen (Gürlevik et al. 2016, 12). Allgemeines Interesse an Politik scheint bei der Mehrheit der Jugendlichen in Österreich vorhanden zu sein (Großegger/Rohrer 2015). Es besteht sogar, wie einleitend erwähnt, aus Sicht vieler Jugendlicher ein Wunsch, sich in der Schule mehr mit politischer Bildung zu befassen (Filzmaier 2007; SORA/Zandonella 2018).

Auch wenn der Zugang junger Menschen zur Beschäftigung mit Demokratie durch die Gleichsetzung von Politik mit Parteipolitik verstellt ist und Jugendliche oftmals wenig Vorstellung davon haben, dass Demokratie ihren eigenen Alltag betrifft, legen die genannten Forschungsergebnisse nahe, dass 
viele Jugendliche der Behandlung von Themen wie Freiheit, Gleichheit oder Solidarität gegenüber aufgeschlossen sind. Dies deckt sich mit den Erfahrungen der an unserem Projekt beteiligten Wissenschaftler"innen und Vermittler*innen. Wenn jungen Menschen Desinteresse an Politik attestiert wird, dann wird meist ausgeblendet, dass Fragen nach persönlichen Freiheitsspielräumen, nach Gleichbehandlung und nach Gruppensolidarität einen zentralen Stellenwert im Alltagsleben von Jugendlichen einnehmen. Denn individuelle Autonomie, soziale Gerechtigkeit (vor allem im Sinne von Gleichberechtigung) und Zusammenhalt sind für Jugendliche wesentliche Kategorien, um das eigene Handeln in seinen Möglichkeiten und Begrenzungen zu verstehen. Freiheit, Gleichheit und Solidarität werden also als zentrale Grundwerte der Demokratie nicht allein im politischen System verhandelt, sondern auch im Alltag der Menschen - und zwar laufend neu. Ein funktionierendes demokratisches System, so die Erkenntnis der politischen Kulturforschung (Almond/Verba 1989; Fuchs 2007), benötigt eine solche Verankerung demokratischer Praktiken und Einstellungsinhalte im alltagskulturellen Leben der Menschen. Auf diese Weise wird Demokratie "gemacht" (Ebner von Eschenbach 2016).

Making Democracy untersuchte nun gemeinsam mit den Jugendlichen Beispiele für alltägliche Aushandlungsformen demokratischer Grundwerte, mit einem Fokus auf den Ort Schule. Im Zentrum stand die Frage, wie sich im Leben der Jugendlichen demokratische Grundfragen von persönlicher Autonomie und deren Einschränkung, von Gleichheit und Ungleichbehandlung, von Solidarität und Ausschluss stellen. Ziel unseres Projekts war nicht die Vermittlung demokratischer Werte, sondern ein Erforschen der Dimensionen und Konkretisierungen dieser Werte im Alltag der Jugendlichen.

In jedem Schuljahr wurden die Jugendlichen im Rahmen von circa 15 mehrstündigen, großteils halbtägigen Workshops von einem Team aus Wissenschaftler*innen, Künstler*innen, Kunstvermittler*innen und Lehrer*innen dabei begleitet, eigene Fragen zu den Themen Gleichheit, Freiheit und Solidarität zu formulieren und diesen mit sozialwissenschaftlichen Methoden wie Interviews, Fragebögen etc. sowie künstlerischen Methoden wie Theaterarbeit, Comics oder Radio nachzugehen. Die Ausarbeitung der Themen und Fragestellungen fand dabei im Sinne partizipativer Forschung in einem kollaborativen Prozess gemeinsam mit den Schüler"innen statt. Am Anfang wurden Zugänge zum Themenkomplex Demokratie im Alltag entwickelt und gleichzeitig die Schule und andere Alltagskontexte, die die 
Schüler*innen einbrachten, erkundet. Ausgehend von der Frage nach den Implikationen und Möglichkeiten alltäglicher demokratischer Praxen generierten sie im Wechsel zwischen Plenum und Kleingruppe sowie zwischen Aktion und Reflexion ihre konkreten Fragen und Anliegen. Hierbei spielte methodisch die eigene Gestaltung von Produkten wie Zines, Kurzvideos und Miniperformances, die den Fragen und Ideen der Jugendlichen eine Gestalt gaben, eine wichtige Rolle, um den Prozess der Reflexion im Tun zu ermöglichen. In der Zusammenarbeit in Kleingruppen verschränkten sich die jeweils eigenen Fragen und die Aushandlungsprozesse in der Gruppe mit der Praxis, etwas gemeinsam im Kollektiv zu artikulieren.

Das Projektteam stellte Methoden sozialwissenschaftlicher Forschung vor und involvierte die Jugendlichen im Sinne kritischer Kunstvermittlung in die Diskussion, Produktion und Reflexion von Bildern. Gemeinsam mit den Jugendlichen wurden Präzisierungen ihrer Themen und Fragestellungen erarbeitet. In den Kleingruppen wurden dann die Methoden für die jeweiligen Forschungsfragen und -settings adaptiert. Die von den Schüler*innen erhobenen Daten wurden von den Wissenschaftler*innen zeitnah verschriftlicht und für die gemeinsame Analyse in der Gruppe sowie für die Weiterarbeit zur Verfügung gestellt.

Die Konzeption der Workshops erfolgte prozessorientiert in Sitzungen des interdisziplinär zusammengesetzten Projektteams (Politikwissenschaft, Soziologie/Psychologie, Kunst- und Kulturvermittlung). In regelmäßigen Abständen waren auch die am Projekt beteiligten Lehrkräfte oder zumindest die Klassenvorstände an den Vor- und Nachbesprechungen der Workshops beteiligt. Die Schüler*innen hatten mittels Zwischenreflexionen und Feedbacks die Möglichkeit, auf die inhaltliche und methodische Gestaltung des Prozesses Einfluss zu nehmen, nur sehr eingeschränkt jedoch auf die Rahmenbedingungen, wie den Zeitrahmen, was insbesondere im zweiten Schuljahr selbst zum Gegenstand der Auseinandersetzung wurde (siehe den Beitrag von Nora Landkammer in diesem Band).

Die Workshop-Reihen beinhalteten in jedem Schuljahr eine Phase der Recherche und Themenfindung und eine Phase für Erhebungen und zur Generierung von Zwischenergebnissen. In einer dritten Phase wurde an der Umsetzung der Ergebnisse in verschiedene Medien gearbeitet. Darauf folgten jeweils eine öffentliche Präsentation und eine gemeinsame Reflexion.

Eine Methode, um im gemeinschaftlichen Forschungsprozess - selbst in einer sehr großen Gruppe (im ersten Schuljahr waren über 50 Schüler*in- 
nen beteiligt) - über die Arbeiten und Zugänge der anderen informiert zu bleiben und ein Ineinandergreifen der inhaltlichen Fragen aus den jeweiligen Workshops zu gewährleisten, war das Festhalten von Zwischenergebnissen in unterschiedlichen Formaten (Plakate, Karten, Statistiken, Bilder und Videos). Auf diese wurde dann in den darauffolgenden Workshops zurückgegriffen.

Ausgehend von der Bearbeitung der Forschungsfragen mit sozialwissenschaftlichen und künstlerischen Methoden erfolgte schließlich die Planung für die Präsentation der Ergebnisse bei einer Abschlussveranstaltung. Im ersten Jahr fand diese im Rahmen einer Abendveranstaltung im Dschungel Wien, einem Theaterhaus für junges Publikum, statt; im zweiten Jahr präsentierten die Jugendlichen ihre Ergebnisse bei der Abschlusstagung im Volkskundemuseum Wien. Die gewählten Darstellungsformen waren im ersten Projektjahr ein Forschungswagen, auf dem der Forschungsprozess sichtbar gemacht wurde, lebensgroße Comicfiguren, die in Sprechblasen Forderungen der Schüler*innen transportierten, und Performances, in denen die Schüler*innen ihre Fragestellungen verhandelten, und im zweiten Jahr Präsentationen und Videoarbeiten. Die Darstellungsformen dienten nicht nur zur Veranschaulichung von bereits existierenden Ergebnissen, sie wurden selbst als Medien einer forschenden Auseinandersetzung verstanden. Die Beschäftigung mit Fragen der Repräsentation eröffnete gleichzeitig die Möglichkeit, die eigenen Forschungsfragen noch einmal neu zu denken.

\section{Aufbau des Buches}

Der erste Abschnitt des vorliegenden Sammelbands bezieht sich auf die eingangs skizzierten Überlegungen zum Projekt Making Democracy und expliziert diese ausgehend von unterschiedlichen Fragestellungen. Die Reflexionen und Auswertungen tragen zu einer selbstkritischen Verortung des Projekts bei und ermöglichen Schlüsse für zukünftige Bildungs- und Vermittlungsprozesse. Darauffolgend werden in einem zweiten Abschnitt, ausgehend von der Tagung im Mai 2019, die Aushandlungen demokratischer Grundwerte in unterschiedlichen Vermittlungskontexten über das Projekt hinaus diskutiert. Verschiedene theoretische Perspektiven auf die Aushandlung von Demokratie im Alltag ergänzen die Einblicke in die Praxis.

Oliver Marchart erläutert mit seinem Beitrag Aspekte der politik-theoretischen Betrachtung von Demokratie im Alltag. Damit werden Überlegungen 
benannt, die das Untersuchungsdesign des Projekts Making Democracy maßgeblich prägten. So fragt Marchart, wie sich in unserem Alltag Freiheit, Gleichheit und Solidarität widersprechen, überkreuzen und stets neu verhandelt werden. Zugespitzt auf den Projektkontext wird die Rolle der Schule als ein Ort der alläglichen Aushandlung von Demokratie diskutiert.

Elke Rajal zeigt, wie die Aushandlungsprozesse von Freiheit, Gleichheit und Solidarität unter Jugendlichen im Projekt Making Democracy konkret stattfanden und $\mathrm{zu}$ welchen thematischen Schwerpunktsetzungen diese führten. Der Beitrag untersucht, wie diese großen Begriffe mit konkreten Bedeutungen gefüllt wurden. Ebenso wird nachgezeichnet, welche Erkenntnisse aus sozialwissenschaftlicher Perspektive für Bildungs- und Demokratisierungsprozesse gezogen werden können.

Dass die jugendlichen Co-Forscher*innen das Projekt Making Democracy selbst unter dem Gesichtspunkt von Demokratie und Mitbestimmung kritisierten, ist Thema des Beitrags von Nora Landkammer. Welche Verständnisse von Demokratie, welche Forderungen werden sichtbar, wenn Jugendliche, Forscher"innen, Vermittler"innen und Lehrer"innen über einen partizipativen Prozess in institutionell und strukturell eingeschränkten Rahmenbedingungen verhandeln? Landkammer arbeitet heraus, wie das Projekt Kristallisationspunkt für Forderungen nach Mitbestimmung im schulischen Kontext und darüber hinaus wurde und zugleich Raum schuf, solche Forderungen zu entwickeln und zu diskutieren.

Aus der Perspektive der Lehrer*innen besprechen Beate Wallner und Stefanie Schermann die Projektidee und ihre Umsetzung am Schulstandort antonkriegergasse. Ihr Beitrag setzt sich mit dem komplexen Verhältnis zwischen den Inhalten, den Methoden und den Visionen der unterschiedlichen Projektbeteiligten auseinander. Die beiden Lehrerinnen reflektieren die sozialen Beziehungen und die Zusammenarbeit des Forschungsteams und aller Projektbeteiligten sowie die schulischen Rahmenbedingungen und ihre pädagogische Motivation im gelebten schulischen Alltag.

Aslı Kışlal, Gründerin des am Projekt Making Democracy beteiligten Theater-, Film- und Performancelabors diverCITYLAB, verhandelt die (Un-) Möglichkeit eines demokratischen Lernens und eines demokratischen Theaters. Ausgehend von undemokratischen und ungleichen Machtverhältnissen in der Gesellschaft - die auch in der Schule sowie im Theater präsent sind - fragt die Autorin, ob und wie diese Räume demokratisierbar sind. Im Vergleich der beiden Lehr- und Lernorte beschäftigt sie sich mit den 
Bedingungen der diversen Versuche, kollektive Übungsräume für Freiheit und für eine demokratische Utopie zu schaffen.

Renate Höllwart, Elke Smodics und Nora Landkammer loten die Schnittstelle zwischen kritischer Kunstvermittlung und partizipativen Forschungsmethoden aus, die für die Gestaltung des Projekts grundlegend war. Sie konkretisieren diese Schnittstelle an Beispielen aus der Vermittlungs-/Forschungspraxis. Auch wenn beiden Traditionen innewohnt, die Grenzen der Wissenschaft, der Kunst und die Autorität der Wissenschaftler*in sowie der Pädagog*in zu untergraben, plädieren die Autor*innen dafür, Lernen, Forschen und die Widerständigkeit von Kunst als wesentliche Elemente für partizipative Prozesse im Blick zu behalten.

Eine der vier Forschungsgruppen der Schüler*innen im zweiten Projektjahr sammelte Fragen und Antworten zum Thema »Freiheit in der Schule«, woraus sie in Zusammenarbeit mit diverCITYLAB ein Video produzierten. Diese Selbstbefragung ist ein weiterer Beitrag des Sammelbandes.

Auf die Dialoge der Jugendlichen folgt eine Bildstrecke, koordiniert von Renate Höllwart und Elke Smodics. Die fotografische Zusammenstellung bildet den Prozess von Making Democracy in der Schule visuell ab.

Im zweiten Abschnitt werden aus verschiedenen Perspektiven Vermittlungsprozesse - vorwiegend im Kontext Schule - als politische Felder im Kampf um Hegemonie diskutiert. Gemein ist den Texten der Fokus auf demokratische Aushandlungsprozesse im Alltag, die eine Auseinandersetzung um das Politische implizieren und Überlegungen zum Potenzial von Vermittlung im Allgemeinen eröffnen. Die Frage nach gesellschaftlicher Veränderung ist im Zuge dieser Überlegungen ein zentrales Leitmotiv.

Karin Schneider diskutiert das Handlungs- und Methodenrepertoire der Kunstvermittlung. Ausgehend von der Reflexion ihrer Erfahrungen in zwei Sparkling-Science-Projekten zielt sie darauf ab, die eigenen Handlungsweisen als Forscherin beziehungsweise Vermittlerin zu verstehen. Mit diesem kritischen und erfahrungsbasierten Text zeigt sie die Möglichkeiten von Vermittlungsprozessen auf. Im Zentrum stehen dabei Ausschlüsse und die Frage, was das Methodenrepertoire damit zu tun hat, diese zu reproduzieren oder zum Thema zu machen.

Nach einer Einführung in das Feld der Kulturvermittlung und einer Reflexion der Rolle der Forscher*in schildert Elke Zobl fortwährende Widersprüche in der kritischen Vermittlungspraxis. Ihr Beitrag stellt zwei weitere Projekte vor, die vor allem auf partizipativen Do-it-yourself-Praktiken auf- 
bauen. DIY und DIT (Do it together) werden als Interventionen in herrschende soziale und kulturelle Ordnungen begriffen, um Handlungsräume für Jugendliche $\mathrm{zu}$ eröffnen und Möglichkeiten der gesellschaftlichen Mitgestaltung erfahrbar zu machen.

Claudia Hummel bietet in ihrem Beitrag eine historisch-politische Verortung des Begriffs der Kulturarbeit. Entlang der Geschichte des Instituts für Kunst im Kontext an der Universität der Künste Berlin zeichnet sie nach, wie Kulturarbeit als Schlüsselbegriff für Kunst als befreiende Tätigkeit für alle fungierte. Vorwiegend im Zusammenhang mit politischen Kämpfen um Arbeit »machten« Kunstvermittler*innen Demokratie, indem sie versuchten, demokratische Arbeits- und Aushandlungsweisen zu entwickeln und künstlerische Mittel dafür zu demokratisieren. Diese historische Einbettung soll schließlich eine kritische Verortung von aktuellen Kulturvermittlungsprojekten ermöglichen.

Demokratische Aushandlungen im Alltag sind eng verknüpft mit der Herstellung von gesellschaftlichem Konsens - und damit von Hegemonie. Die Rolle von Pädagogik und Vermittlungsprozessen untersucht Ingo PohnLauggas mit Rückgriff auf Antonio Gramscis Begriff des Alltagsverstands und seiner Konzeption von Hegemonie als pädagogischem Verhältnis. Dabei diskutiert der Autor die im Buch aufgeworfene Frage der Verhandlung demokratischer Grundwerte als Beispiel für eine Kohärenzpraxis im Alltag.

Der Beitrag von das kollektiv plädiert für kritische Bildungskonzepte und -praxen als Kämpfe um emanzipatorische Veränderung und rekurriert auf die alltäglichen Erfahrungen in der Erwachsenenbildung. Die Autorinnen* gehen von einer Zunahme von Ungleichheit in einer postkolonialen und postnationalsozialistischen (Welt-)Gesellschaft aus, die auf einer Tradition "trügerischer Inklusion« von Migrierten und Geflüchteten aufbaut. So fragen sie »Wozu lernen? = Wozu kämpfen? « und adressieren das Verhältnis des Globalen Nordens zum Globalen Süden sowie die Möglichkeit von solidarischen Verbindungen unterschiedlicher politischer Kämpfe. Die Organisation das kollektiv begreift sich selbst als einen Ort kritischer Bildungsarbeit für migrierte und geflüchtete Frauen* - so kommen im Beitrag auch Kursteilnehmerinnen* als zentrale Akteurinnen" des gemeinsamen Lernens zur Sprache.

Rahel Süß untersucht aus der Perspektive radikaler Demokratietheorie das Erlernen von Demokratie und seine möglichen Formen. Als konstitutiv für demokratische Prozesse betont sie die Möglichkeit von Handlungen, die 
auf Veränderungen und eine notwendigerweise ergebnisoffene Zukunft gerichtet sind. Demokratie funktioniert dabei der Autorin zufolge als radikaler Experimentalismus. Entsprechend bestimmt sie das Experiment als einen demokratischen Schlüsselbegriff. Liberale Demokratien seien insofern auf Experimente angewiesen, als dass sie das Potenzial haben, die Zukunft radikal offenzuhalten.

Als Abschluss des Bandes wirft Nora Sternfeld die Frage auf, wie eine Demokratie gelernt werden kann, die es noch nicht gibt. So tragen Lern- und Vermittlungsprozesse als Mechanismen von Herrschaft doch zur Persistenz hegemonialer Verhältnisse bei. Anschließend an Gramscis Überlegungen zum pädagogischen Verhältnis eröffnet die Autorin Möglichkeitsräume im Denken über Veränderung, die ein Verlernen von Machtverhältnissen und eine Auseinandersetzung mit der Idee der Gleichheit aller Menschen zulassen. Damit wird Gleichheit als Heterogenität zum Ausgangspunkt und zugleich zur Forderung.

Die Beiträge im Band thematisieren demnach nicht nur Vermittlungsund Bildungsprozesse als Austragungsort der Kämpfe um Hegemonie, sondern auch das Wie dieser Prozesse.

\section{Literatur}

Almond, Gabriel Abraham/Verba, Sydney (1989). The Civic Culture. Political Attitudes and Democracy in Five Nations. Thousand Oaks: SAGE Publications.

Ebner von Eschenbach, Malte (2016). Doing Difference - Die Reflexion auf Unterscheidungen als Ansatz Politischer Erwachsenenbildung, in: Magazin erwachsenenbildung.at. Das Fachmedium für Forschung, Praxis und Diskurs. Nr. 28, abrufbar unter: https://www.erwachsenenbildung. at/magazin/ausgabe-28/10035-doing-difference-die-reflexion-auf-un terscheidungen-als-ansatz-politischer-erwachsenenbildung.php (letzter Zugriff: 19.9.2019).

Filzmaier, Peter (2007). Jugend und politische Bildung. Einstellungen und Erwartungen von 14- bis 24-Jährigen. Kurzbericht zur Pilotstudie (Donau-Universität Krems), abrufbar unter: https://www.donau-uni.ac.at/ dam/jcr:a18537c6-0280-4407-9265-6f15b879efo7/HP_SO_NP_Pilotstudie. pdf (letzter Zugriff: 18.9.2019). 
Fuchs, Dieter (2007). The Political Culture Paradigm, in: Dalton, Russell J./ Klingemann, Hans-Dieter (Hg.): The Oxford Handbook of Political Behaviour. Oxford: Oxford University Press, 161-184.

Großegger, Beate/Rohrer, Matthias (2015). Jugend und Politik - Repräsentativumfrage unter 14- bis 18-jährigen ÖsterreicherInnen. Eigenstudie des Instituts für Jugendkulturforschung.

Gürlevik, Aydin/Hurrelmann, Klaus/Palentien, Christian (2016). Jugend und Politik im Wandel?, in: dies. (Hg.): Jugend und Politik: Politische Bildung und Beteiligung von Jugendlichen. Wiesbaden: Springer VS, 1-24. https://doi.org/10.1007/978-3-658-09145-3_1.

Oerter, Rolf (2016). Psychologische Aspekte. Können Jugendliche politisch mitentscheiden?, in: Gürlevik, Aydin/Hurrelmann, Klaus/Palentien, Christian (Hg.): Jugend und Politik: Politische Bildung und Beteiligung von Jugendlichen. Wiesbaden: Springer VS, 69-84. https://doi.org/10.1007/978-3-65809145-3_4.

Palentien, Christian (2016). Erziehungswissenschaftliche Betrachtung. Mitwirkung, Interesse und Lernmotivation in der Schule, in: Gürlevik, Aydin/Hurrelmann, Klaus/Palentien, Christian (Hg.): Jugend und Politik: Politische Bildung und Beteiligung von Jugendlichen. Wiesbaden: Springer VS, 103-114. https://doi.org/10.1007/978-3-658-09145-3_6.

Shell Jugendstudie (2015). Wie tickt die Jugend?, abrufbar unter: https:// www.shell.de/ueber-uns/die-shell-jugendstudie/jugend-und-politik. html (letzter Zugriff: 29.8.2019).

SORA (Institute for Social Research and Consulting)/Zandonella, Martina (2018). Österreichischer Demokratie Monitor, abrufbar unter:https://www. demokratiemonitor.at/wp-content/uploads/2018/11/2018_Pr\%C3\%A4sen tation_\%C $3 \% 96 s t e r r e i c h i s c h e r \_D e m o k r a t i e \_M o n i t o r . p d f$ (letzter Zugriff: 29.8.2019).

Soßdorf, Anna (2016). Zwischen Like-Button und Parteibuch. Die Rolle des Internets in der politischen Partizipation Jugendlicher. Wiesbaden: Springer VS. https://doi.org/10.1007/978-3-658-13932-2. 



\title{
Demokratie im Alltag Anmerkungen zum politik-theoretischen Untersuchungsdesign von Making Democracy
}

Oliver Marchart

\begin{abstract}
Dieser Beitrag behandelt die politik-theoretischen Überlegungen zu den demokratischen Grundprinzipien Freiheit, Gleichheit und Solidarität und ihren Zusammenhang, die das Untersuchungsdesign des Projekts Making Democracy prägten. Die Schule wird als ein Ort der alltäglichen Aushandlung von Demokratie im Alltag diskutiert.
\end{abstract}

Seit den Zeiten der Französischen Revolution ist Demokratie an der Devise von Freiheit, Gleichheit und Solidarität ausgerichtet. Auch heute noch definiert diese Trias die Grundnormen der Demokratie. Das bedeutet freilich nicht, dass letztere in ihrer Bedeutung unumstritten wären. Politische Debatten drehen sich oftmals um den Stellenwert, der ihnen zuzuschreiben ist (was etwa die Einführung von Wertetests für neu Zugewanderte zeigt). Genauso wenig herrscht Konsens bezüglich des Einzugsbereichs der jeweiligen Grundnormen. Wer gilt in liberalen westlichen Demokratien als frei und gleich? In welcher Hinsicht gilt man als frei und gleich? Wer kann Anspruch auf Solidarität geltend machen? Und auf welche Art von Solidarität? Es ist also keineswegs ausgemacht, was genau unter Freiheit, Gleichheit oder Solidarität zu verstehen ist. Dennoch sind diese Prinzipien im demokratischen Diskurs omnipräsent. Selbst wo sie nicht explizit zum Gegenstand politischer Auseinandersetzung werden, bilden sie doch immer die implizit anerkannte Referenzfolie.

$\mathrm{Zu}$ den Aufgaben politischer Theorie zählt daher die begriffliche Klärung jener Grundprinzipien, die den Horizont demokratischen Handelns bilden. 
Dabei stieß die jüngere Demokratietheorie auf das Problem, dass diese Prinzipien nur schwer miteinander vereinbar sind, da sie unterschiedlichen politischen Traditionen und Ideologien entstammen (Bobbio 1990). Für Chantal Mouffe (2008) stehen Freiheit und Gleichheit in einem paradoxen Verhältnis. Freiheit entstamme, so Mouffe, der liberalen Tradition und beziehe sich auf die liberalen Freiheitsrechte des Individuums, das Prinzip der Rechtsstaatlichkeit und den Schutz der Menschenrechte. Gleichheit wiederum basiere auf der demokratischen Tradition im engeren Sinn und beziehe sich auf das Prinzip der Volkssouveränität und das Majoritätsprinzip, beinhalte aber nicht den Schutz individueller Rechte. Die moderne liberale Demokratie sei deshalb als ein Kompromiss zwischen zwei letztlich unvereinbaren Werten zu verstehen, die dennoch in ein instabiles Gleichgewicht gebracht werden müssten. ${ }^{1}$ Solche demokratietheoretischen Überlegungen werden im Regelfall keiner empirischen Überprüfung unterzogen, sondern innertheoretisch postuliert und ideengeschichtlich gestützt. Das bedeutet jedoch nicht, dass sie sich nicht in ein empirisches Untersuchungssetting übersetzen ließen. Doch wo ansetzen?

Ein möglicher Ansatzpunkt ergibt sich, wenn wir berücksichtigen, dass demokratische Grundprinzipien nicht nur in abstrakten theoretischen Traktaten, in Parteiprogrammen oder Politikerreden, sondern auch im Alltag der Menschen verhandelt werden. Demokratie wird "gemacht« (Ebner von Eschenbach 2016). Natürlich nicht ein für alle Mal, sondern Demokratie bleibt »in the making", und zwar sowohl innerhalb des politischen Systems als auch im Feld der Alltagskultur beziehungsweise des "senso comune oder »Alltagsverstands« der Menschen (Gramsci, vgl. den Beitrag von Pohn-Lauggas in diesem Band). In unserem Alltagsverstand verfügen wir alle über ein implizites Verständnis von legitimen Freiheitsspielräumen und plausiblen Ansprüchen auf Gleichbehandlung und Solidarität. Wo wir diese Ansprüche verletzt sehen, reagieren wir wütend, indigniert oder beschämt, ohne uns womöglich Rechenschaft darüber abzulegen, dass nicht nur individuelle, sondern demokratische und damit allgemeinverbindliche Ansprüche verletzt wurden. Zugleich ist im Alltag - und vielleicht weniger noch als im politischen System - keineswegs festgelegt, worin genau diese Ansprüche bestehen, da die Frage, welche demokratische Grundnorm in welcher Weise zu tragen kommt, zumeist nur implizit mitverhandelt wird.

1 Für die entgegengesetzte Hypothese der Gleichursprünglichkeit, wenn nicht Identität von Freiheit und Gleichheit in Gestalt der »Cleichfreiheit«vgl. etwa Balibar (2012). 
Das Forschungssetting, das für Making Democracy entwickelt wurde, erlaubt es, die politisch-theoretische Frage nach den demokratischen Grundnormen in einen produktiven Bezug zu qualitativer empirischer Forschung zu bringen. Den Hintergrund unserer Untersuchungen bilden Erkenntnisse der Demokratieforschung (vor allem in Bezug auf Jugendliche) und neueren politischen Theorie (insbesondere radikaldemokratische Theorien) sowie Ansätze der Cultural Studies, der Migrationspädagogik und der Intersektionalitätsforschung. Indem Making Democracy an Alltagssituationen - insbesondere im Schulbereich - ansetzt, verschiebt das Projekt den Fokus der Demokratieforschung: Gefragt wird nicht nach dem Verhältnis Jugendlicher zum politischen System (insbesondere zu Wahlen und Parteipolitik), sondern nach dem in der Alltagskultur verankerten demokratischen Wertekanon der Jugendlichen. Denn obwohl zahlreiche Befunde deutlich machen, dass Beteiligungs- und Mitgestaltungsmöglichkeiten für das Demokratiebewusstsein von Jugendlichen zentral sind (Oerter 2016, 81; Palentien 2016, 112), stellt eine solche Refokussierung immer noch ein Desiderat der Demokratieforschung dar (Gürlevik et al. 2016, 8, 12).

Allerdings stehen wir hier vor dem bereits angedeuteten Problem: Fragen rund um Freiheit, Gleichheit und Solidarität werden im Alltagsleben von Jugendlichen selten als explizit (demokratie-)politische Fragen aufgeworfen. Obgleich implizit als grundlegend bedeutsam vorausgesetzt, werden sie vor allem ex negativo thematisiert. Dies auch deshalb, weil Jugendliche tagtäglich in verschiedenen Kontexten mit Erfahrungen des Zwangs, der Ungleichheit und des Ausschlusses konfrontiert sind: erstens in der Familie vor dem Hintergrund der Neudefinition ihrer Rolle in der Pubertät, zweitens in der Schule, wo sie dazu angehalten sind, für ihre Zukunft zu lernen und dabei hierarchischen Strukturen ausgesetzt sind, drittens im Freundeskreis, in dem sich Fragen nach Freundschaft, Zusammenhalt und Konkurrenz auf vielfache Weise stellen, sowie viertens in virtuellen Foren, in denen Dynamiken des Ein- und Ausschlusses eine zentrale Rolle einnehmen und auf reale Räume rückwirken. Jugendliche sind in all diesen Kontexten herausgefordert, Vorstellungen und Praktiken von Freiheit, Gleichheit und Solidarität für sich zu erarbeiten und in Interaktion mit anderen auszuloten.

Doch welche Rolle kommt der Schule als einem Ort der Aushandlung von Demokratie zu? Zahlreiche Studien und Forschungsprojekte haben gezeigt, dass binäre Logiken (etwa Unterscheidungen in "zugehörig" und "nicht zugehörig« oder die Logik der Zweigeschlechtlichkeit) und Normierungen 
im Unterricht immer noch bestimmend sind und Heterogenität in all ihren Formen zu wenig berücksichtigt wird (Holzkamp 1995; Mecheril 2004; Trautmann/Wischer 2011). Lern- und Aushandlungsprozesse werden auf diese Weise eingeschränkt. Lernen findet vor allem als »defensives Lernen« (Holzkamp 1995) statt und wird so häufig seines Potenzials, Veränderungsprozesse anzustoßen, entledigt. Zudem erschwert es das österreichische Bildungssystem Kindern und Jugendlichen aus nichtprivilegierten, insbesondere aus migrantischen Familien, ihre Potenziale auszuschöpfen (Dirim et al. 2010; Schwantner/Schreiner 2010, 52). Eine wesentliche Ursache besteht in der Reproduktion sozialer Ungleichheiten durch die Bildungsinstitutionen selbst (Bourdieu/Passeron 1971; Gomolla/Radtke 2009; Mecheril 2010; HerzogPunzenberger/Schnell 2012).

Sie funktioniert weitgehend über die Behauptung der Neutralität von Leistungskriterien, was die Bedeutung von kulturellem, sozialem und symbolischem Kapital ${ }^{2}$ ignoriert, die Rolle des Habitus ${ }^{3}$ ausblendet und damit selektive Unterscheidungen erst hervorruft und - vielfach über die Begabungsideologie - legitimiert (Erler 2011; Liebau 2011). ${ }^{4}$ Da die über den Habitus vermittelte Herstellung von Unterschieden wenig bewusst erfolgt, bleibt sie als Machtverhältnis weitgehend unsichtbar (Leimstättner 2011). Hinzu kommt, dass die Rechte auf Mitgestaltung und Mitbestimmung, die in der UN-Kinderrechtskonvention ${ }^{5}$ und auch deutlich im österreichischen Schulunterrichtsgesetz festgeschrieben sind, ${ }^{6}$ in der Schule selten wirklich ausgeschöpft werden.

2 Pierre Bourdieu unterscheidet vier Formen von Kapital: Mit ökonomischem Kapital ist der materielle Reichtum gemeint; kulturelles Kapital bezeichnet kulturelle Fähigkeiten, Fertigkeiten und Wissensformen sowie Bildungstitel; soziales Kapital meint den Zugriff auf ein Netzwerk von Akteur*innen, eine Ressource, die auf der Zugehörigkeit zu einer Cruppe beruht; symbolisches Kapital ist eine den drei zuvor genannten Kapitalformen übergeordnete Ressource, welche durch gesellschaftliche Anerkennungsakte zustande kommt (Abels/König 2010, 205-207).

3 Bezugnehmend auf Bourdieu versteht man unter Habitus eine klassenspezifische Art zu denken und zu handeln, die vor allem auf das kulturelle Kapital zurückgreift (Abels/König 2010, 206).

4 Vgl. für die folgenden Ausführungen auch Rajal et al. 2019.

5 Zentral sind in unserem Kontext die Artikel 12 (Berücksichtigung des Kindeswillens) und 13 (Meinungs- und Informationsfreiheit) der UN-Kinderrechtskonvention.

6 »Der Schüler hat außer den sonst gesetzlich festgelegten Rechten das Recht, sich nach Maßgabe seiner Fähigkeiten im Rahmen der Förderung der Unterrichtsarbeit (§43) an der 
Das Projekt Making Democracy stützt sich daher auf Erkenntnisse dreier Forschungsfelder, die bei der Untersuchung der Aushandlung demokratischer Grundwerte durch Schüler*innen im Kopf behalten werden müssen: Migrationspädagogik, Cultural Studies und Intersektionalitätsforschung. Die Perspektive der Migrationspädagogik (Mecheril 2004; Mecheril et al. 2010; Mecheril 2012) problematisiert Unterscheidungspraxen und erlaubt es, die "phantasmatische Erzeugung von >Wir und >Nicht-Wir« (Mecheril 2013) im Kontext sozialer und gesellschaftlicher Machtverhältnisse $\mathrm{zu}$ untersuchen (Broden/Mecheril 2007, $7 \mathrm{f}$.), um auf diese Weise Hemmnisse für Bildungsprozesse zu erkennen und Fragen von Gleichheit und Ungleichheit zu bearbeiten (Mecheril 2011, 26). Paul Mecheril formulierte hierfür die folgenden migrationspädagogischen Leitlinien: Erstens die Ermöglichung der Handlungsfähigkeit aller, zweitens die Differenzfreundlichkeit und $\mathrm{Zu}$ schreibungsreflexivität (also einen offenen, positiven Umgang mit Differenzen und gleichzeitige Reflexion der Tatsache, dass Prozesse der Festschreibung von Differenz immer auch mit Zuschreibungen verbunden sind) sowie drittens die Anerkennung der Komplexität von Bildungsprozessen (Mecheril 2013, 10).

Auch in der Perspektive der Cultural Studies wird mit einem dynamischen Kulturbegriff gearbeitet (Kalpaka/Mecheril 2010, 94), um den Herstellungsprozess von Kultur und Handlungsmöglichkeiten sichtbar zu machen. Kultur wird als ein aktiver Prozess der Ausverhandlung der eigenen Position unter von Machtverhältnissen bestimmten sozialen Bedingungen betrachtet (Hall 1994; Sauter 2006; Marchart 2008). Schule kann aus dieser Perspektive als ein Ort begriffen werden, der zwar disziplinierend und akkommodierend ${ }^{7}$ wirkt, zugleich jedoch von Prozessen des Widerspruchs und Widerstands durchzogen ist (Giroux 1996). Auf diese Weise lässt sich die Verbindung von Kultur, Wissen und Macht produktiv bearbeiten und in emanzipatorische Strategien übersetzen (Sauter 2006, 115).

Cestaltung des Unterrichtes und der Wahl der Unterrichtsmittel zu beteiligen, ferner hat er das Recht auf Anhörung sowie auf Abgabe von Vorschlägen und Stellungnahmen« ( $(57 \mathrm{a}$ Schulunterrichtsgesetz: BCBI. Nr. 472/1986).

7 Akkommodation meint nach Jean Piaget einen Prozess der Anpassung der inneren Welt durch Schaffen eines neuen Wahrnehmungsschemas, kognitive Schemata werden also an die Umwelt angeglichen. Im Unterschied dazu bezeichnet Assimilation einen Prozess, bei dem äußere Reize vornehmlich in bestehende kognitive Schemata integriert werden (McLeod 2018). 
Wie die Migrationspädagogik und die Cultural Studies befasst sich auch der dritte Ansatz, die Intersektionalitätsforschung, mit der kritischen Analyse sozialer Ungleichheiten und Machtverhältnisse. Das Konzept der Intersektionalität beschreibt das Zusammenwirken unterschiedlicher Kategorien, die konstitutiv für soziale Positionierungen und damit Ungleichheit sind. Dabei wird unterstrichen, dass soziale Kategorien wie Geschlecht, soziale Schicht, sexuelle Orientierung, Migrationsgeschichte, Nationalität, Ethnizität oder Behinderung in einem dynamischen Wechselverhältnis zueinanderstehen, also ineinander und aufeinander wirken (Lutz et al. 2013). Audre Lorde (2007) folgend: »There is no such thing as a single-issue struggle because we do not live single-issue lives.«

Vielleicht ließe sich dieses Diktum aus Sicht der Demokratietheorie nicht zuletzt auf die vielfachen Formen der Aushandlung demokratischer Grundnormen übertragen. Eine allein am Prinzip der Freiheit, allein am Prinzip der Gleichheit oder allein am Prinzip der Solidarität ausgerichtete Demokratie existiert nicht. In der Tat ist jede demokratische Grundnorm als solche umstritten und dem Druck konkurrierender politischer Definitionsanstrengungen ausgesetzt. Doch nicht nur das. In unserem Alltag überkreuzen sich die Vektoren von Freiheit, Gleichheit und Solidarität. Die demokratischen Grundnormen stehen in einem unauflöslichen Wechselverhältnis. Sie werden immer alle zugleich verhandelt und situationsspezifisch auf ihre Kompatibilität oder Inkompatibilität hin ausgetestet. Weder sind die demokratischen Grundnormen von Anfang an ident noch sind sie prinzipiell unvereinbar, wie der Blick auf deren alltägliche Ausverhandlung zeigt.

\section{Literatur}

Abels, Heinz/König, Alexandra (2010). Sozialisation. Soziologische Antworten auf die Frage, wie wir werden, was wir sind, wie gesellschaftliche Ordnung möglich ist und wie Theorien der Gesellschaft und der Identität ineinanderspielen. Wiesbaden: VS Verlag für Sozialwissenschaften. https:// doi.org/10.1007/978-3-531-92024-5.

Balibar, Étienne (2012). Gleichfreiheit: politische Essays. Berlin: Suhrkamp. Bobbio, Norberto (1990). Liberalism and Democracy, London/New York: Verso. Bourdieu, Pierre/Passeron, Jean-Claude (1971). Die Illusion der Chancengleichheit. Stuttgart: Klett. 
Broden, Anne/Mecheril, Paul (2007). Migrationsgesellschaftliche Repräsentationen. Eine Einführung, in: dies. (Hg.): Re-Präsentationen. Dynamiken der Migrationsgesellschaft. Düsseldorf: IDA-NRW, 7-28.

Dirim, İnci/Mecheril, Paul/Melter, Claus (2010). Materialien zum Kapitel 6. Umgang mit migrationsbedingter Heterogenität im österreichischen Bildungssystem, in: Mecheril, Paul/Castro Varela, María do Mar/Dirim, İnci/Kalpaka, Annita/Melter, Claus (Hg.): Migrationspädagogik. Weinheim/Basel: Beltz, 22-24.

Ebner von Eschenbach, Malte (2016). Doing Difference - Die Reflexion auf Unterscheidungen als Ansatz Politischer Erwachsenenbildung, in: Magazin erwachsenenbildung.at. Das Fachmedium für Forschung, Praxis und Diskurs, Nr. 28, abrufbar unter: https://erwachsenenbildung.at/magazin/ ausgabe-28/10035-doing-difference-die-reflexion-auf-unterscheidungenals-ansatz-politischer-erwachsenenbildung.php (letzter Zugriff:11.2.2019).

Erler, Ingolf (2011). Bildung - Ungleichheit - symbolische Herrschaft, in: Erler, Ingolf u.a. (Hg.): Wie Bourdieu in die Schule kommt. Analysen zu Ungleichheit und Herrschaft im Bildungswesen. Innsbruck: Studienverlag, 22-36.

Giroux, Henry (1996). Fugitive Cultures. Race, Violence, and Youth. London/ New York: Routledge.

Gomolla, Mechthild/Radtke, Frank-Olaf (2009). Institutionelle Diskriminierung. Die Herstellung ethnischer Differenz in der Schule. Wiesbaden: VS Verlag für Sozialwissenschaften. https://doi.org/10.1007/978-3-53191577-7.

Gürlevik, Aydin/Hurrelmann, Klaus/Palentien, Christian (2016). Jugend und Politik im Wandel?, in: dies. (Hg.): Jugend und Politik: Politische Bildung und Beteiligung von Jugendlichen. Wiesbaden: Springer VS, 1-24. https://doi.org/10.1007/978-3-658-09145-3_1.

Hall, Stuart (1994). Rassismus und kulturelle Identität. Hamburg: Argument. Herzog-Punzenberger, Barbara/Schnell, Philipp (2012). Die Situation mehrsprachiger Schüler/innen im österreichischen Schulsystem - Problemlagen, Rahmenbedingungen und internationaler Vergleich, in: HerzogPunzenberger, Barbara (Hg.): Nationaler Bildungsbericht Österreich 2012, Band 2: Fokussierte Analysen bildungspolitischer Schwerpunktthemen. Graz: Leykam, 229-267.

Holzkamp, Klaus (1995). Lernen. Subjektwissenschaftliche Grundlegung. Frankfurt a. M.: Campus. 
Kalpaka, Annita/Mecheril, Paul (2010). »Interkulturell«. Von spezifisch kulturalistischen Ansätzen zu allgemein reflexiven Perspektiven, in: Mecheril, Paul/Castro Varela, María do Mar/Dirim, İnci/Kalpaka, Annita/ Melter, Claus (Hg.): Migrationspädagogik. Weinheim/Basel: Beltz, 77-98.

Leimstättner, Brigitte (2011). Das Feld Schule und seine Akteur/innen, in: Erler, Ingolf u. a. (Hg.): Wie Bourdieu in die Schule kommt. Analysen zu Ungleichheit und Herrschaft im Bildungswesen. Innsbruck: Studienverlag, 78-87.

Liebau, Eckart (2011). Was Pädagogen an Bourdieu stört, in: Erler, Ingolf u. a. (Hg.): Wie Bourdieu in die Schule kommt. Analysen zu Ungleichheit und Herrschaft im Bildungswesen. Innsbruck: Studienverlag, 10-21.

Lorde, Audre (2007). Sister Outsider, Essays and Speeches. Berkeley: Crossing Press.

Lutz, Helma/Herrera Vivar, Maria Teresa/Supik, Linda (2013). Fokus Intersektionalität. Bewegungen und Verortungen eines vielschichtigen Konzepts. Wiesbaden: Springer VS. https://doi.org/10.1007/978-3-531-19550-6.

Marchart, Oliver (2008). Cultural Studies. Konstanz: UVK.

McLeod, Saul (2018). Jean Piaget's Theory of Cognitive Development, abrufbar unter: https://www.simplypsychology.org/piaget.html (letzter $\mathrm{Zu}$ griff: 11.2.2019).

Mecheril, Paul (2004). Einführung in die Migrationspädagogik. Weinheim/ Basel: Beltz.

Mecheril, Paul (2010). Die Ordnung des erziehungswissenschaftlichen Diskurses in der Migrationsgesellschaft, in: Mecheril, Paul/Castro Varela, María do Mar/Dirim, İnci/Kalpaka, Annita/Melter, Claus (Hg.): Migrationspädagogik. Weinheim/Basel: Beltz, 54-76.

Mecheril, Paul/Castro Varela, María do Mar/Dirim, İnci/Kalpaka, Annita/ Melter, Claus (2010). Migrationspädagogik. Weinheim/Basel: Beltz.

Mecheril, Paul (2011). Ästhetische Bildung. Migrationspädagogische Anmerkungen, in: ifa (Institut für Auslandsbeziehungen an der Hochschule Zürich) u.a. (Hg.): Kunstvermittlung in der Migrationsgesellschaft Reflexionen einer Arbeitstagung, 26-35, abrufbar unter: https://intern. zhdk.ch/fileadmin/user_upload/_imported/fileadmin/data/iae/docu ments/121001_0106-482_RZ_WEB_PublikationKunstvermittlung-Migra tionsgesellschaft.pdf (letzter Zugriff: 11.2.2019). 
Mecheril, Paul (2012). Was ist Migrationspädagogik? Vortrag auf der Fachtagung »Ehrenamtliche Bildungsbeauftragte: interkulturelle Akteure für die Verbesserung der Bildungsintegration«, Bonn.

Mecheril, Paul (2013). Von der interkulturellen zur migrationsgesellschaftlichen Öffnung - rassismuskritische Perspektiven, Vortrag auf dem Symposium des pädagogischen Instituts in München.

Mouffe, Chantal (2008). Das demokratische Paradox. Wien: Turia + Kant.

Oerter, Rolf (2016). Psychologische Aspekte. Können Jugendliche politisch mitentscheiden?, in: Gürlevik, Aydin/Hurrelmann, Klaus/Palentien, Christian (Hg.): Jugend und Politik: Politische Bildung und Beteiligung von Jugendlichen. Wiesbaden: Springer VS, 69-84. https://doi.org/10.1007/978-3-65809145-3_4.

Palentien, Christian (2016). Erziehungswissenschaftliche Betrachtung. Mitwirkung, Interesse und Lernmotivation in der Schule, in: Gürlevik, Aydin/Hurrelmann, Klaus/Palentien, Christian (Hg.): Jugend und Politik: Politische Bildung und Beteiligung von Jugendlichen. Wiesbaden: Springer VS, 103-114. https://doi.org/10.1007/978-3-658-09145-3_6.

Rajal, Elke/Garnitschnig, Ines/Marchart, Oliver (2019). Demokratie im Alltag. Aushandlungen von Freiheit, Gleichheit und Solidarität unter Jugendlichen im Rahmen des Forschungsprojekts »Making Democracy«, in: SWSRundschau, 59. Jahrgang, Heft 2/2019, 181-199.

Sauter, Sven (2006). Die Schule als Kampfplatz und als Aushandlungsraum. Über die soziale Bedeutung des Wissens aus der Perspektive der Cultural Studies, in: Mecheril, Paul/Witsch, Monika (Hg.): Cultural Studies und Pädagogik. Kritische Artikulationen. Bielefeld: transcript, 111-144.

Schwantner, Ursula/Schreiner, Claudia (2010) (Hg.). PISA 2009. Internationaler Vergleich von Schülerleistungen. Erste Ergebnisse Lesen, Mathematik, Naturwissenschaft, abrufbar unter: https://www.bifie.at/wp-content/up loads/2017/05/2010-12-07_pisa-2009-ersteergebnisse.pdf (letzter Zugriff: 11.2.2019).

Trautmann, Matthias/Wischer, Beate (2011). Heterogenität in der Schule. Eine kritische Einführung. Wiesbaden: VS Verlag für Sozialwissenschaften. https://doi.org/10.1007/978-3-531-92893-7. 



\title{
Making Democracy. Aushandlungen von Freiheit, Gleichheit und Solidarität unter Jugendlichen Erkenntnisse aus dem Prozess
}

Elke Rajal

\begin{abstract}
Im Projekt Making Democracy wurden Aushandlungen demokratischer Grundwerte durch Jugendliche mit Jugendlichen gemeinsam untersucht. Dieser Artikel behandelt die Themen und Forschungsfragen, die die Jugendlichen dreier Wiener Schulklassen in Kleingruppen im Rahmen des sehr offen formulierten Überthemas - Freiheit, Gleichheit und Solidarität im Alltag-definierten. Die Bearbeitung durch die Jugendlichen zeigt, wie sich die genannten demokratischen Grundwerte für die Jugendlichen konkretisieren und welche Widersprüche, Ungerechtigkeiten und Kämpfe sie verstehen - und auch verändern - möchten.
\end{abstract}

Was lässt sich aus einer politikwissenschaftlichen Perspektive darüber sagen, wie demokratische Grundwerte von den Jugendlichen der am partizipativen Forschungsprojekt Making Democracy beteiligten Schulklassen ${ }^{1}$ ausgehandelt wurden? Was verknüpften sie mit »Demokratie machen« und welche Fragen interessierten sie? Sowohl aus den Themen der Jugendlichen und ihren Fragen als auch aus dem Prozess ihrer Bearbeitung lassen sich Erkenntnisse über die alltägliche Bedeutung demokratischer Grundwerte ableiten. Diese sollen - fokussiert auf das Verhältnis zwischen Freiheit, Gleichheit und Solidarität (zur Einordnung dieses Verhältnisses siehe den Beitrag

1 Im Schuljahr 2017/18 forschten rund 50 Schüler*innen im Alter von 12 bis 13 Jahren (7. Schulstufe, Wiener Mittelschule antonkriegergasse, Wien) und im Schuljahr 2018/19 circa 18 Schüler*innen im Alter von 16 bis 19 Jahren (11. Schulstufe, Oberstufenrealgymnasium antonkriegergasse, Wien) im Rahmen des Projekts. 
von Oliver Marchart in diesem Band) - in diesem Artikel vorgestellt werden. ${ }^{2}$ Dabei ist $\mathrm{zu}$ beachten, dass die hier veranschaulichten Themen in gruppendynamischen Prozessen entstanden - sie veränderten sich und überkreuzten sich mit Auseinandersetzungen zwischen den Jugendlichen und mit dem Forschungsteam über die Inhalte und den Projektrahmen.

Das Projektdesign sah vor, dass die Schüler*innen eigene Fragestellungen formulieren und dabei die recht abstrakte Frage danach, wie demokratische Grundwerte im Alltag ausgehandelt werden, auf ihr eigenes Leben beziehen. Rund 15 Workshops standen dafür in jedem Schuljahr zur Verfügung. Ziel war es, eine möglichst dichte Beschreibung der Vielgestaltigkeit von Bezügen zu und Aushandlungsformen von demokratischen Grundwerten und damit auch Konkretisierungen der abstrakten Überfrage zu erhalten. So sollten alltagskulturelle Bedeutungen von Freiheit, Gleichheit und Solidarität im Leben der Jugendlichen sichtbar gemacht werden.

Der Datenkorpus aus den beiden Projektjahren umfasst: mehrere Interviews und Befragungen, die die Jugendlichen mit Schüler*innen ihrer und anderer Klassen durchführten; Videos, die den Prozess der Entwicklung der Performances festhalten; Reflexionsprotokolle, die alle Projektbeteiligten am Ende der Workshops zu Papier brachten (1. Schuljahr); Auszüge von Forschungstagebüchern der Schüler*innen (1. Schuljahr) und Forschungstagebücher der Wissenschaftler*innen; Comics, Zines und Plakate, die im Zuge der Workshops entstanden; Fotodokumentationen; Gruppengespräche mit Schüler*innen; Gruppengespräche mit Lehrer*innen und Vermittler*innen usw.

Anhand ausgewählter Materialien aus diesem Korpus zeichne ich im Folgenden das Spektrum der Themen nach und analysiere das Verhältnis, das sich zwischen Freiheit, Gleichheit und Solidarität in der Behandlung der Themen durch die Jugendlichen abzeichnete. Bei der Analyse der jeweiligen Forschungsprozesse rekurriere ich auf die theoretischen Konzepte, wie sie in der Einleitung zu diesem Band sowie im Text von Oliver Marchart dargelegt werden.

2 Die Auswertungen des 1. Schuljahres wurden von Elke Rajal und Ines Garnitschnig durchgeführt, die Auswertungen des 2. Schuljahres von Elke Rajal und Nora Landkammer. Teile der Ergebnisse des 1. Schuljahres sind bereits publiziert in: Rajal/Garnitschnig/Marchart 2019. Zu den Zielen und Methoden des Projekts siehe auch: Garnitschnig 2019. 


\section{Freiheit, Gleichheit und Solidarität in den Themen der Jugendlichen}

Im ersten Projektjahr kristallisierten sich vier Themenbereiche als die zentralen heraus: Freiheit in der Schule, Videospiel, Mobbing und Rassismus. Daran arbeiteten jeweils eine oder sogar mehrere Kleingruppen. Im zweiten Projektjahr bildeten sich ebenfalls vier Themenbereiche, an denen jeweils eine Gruppe arbeitete: Schule und Freiheit, Digitalisierung, Staatsbürgerschaft/ Wahlrecht sowie Sport und Gleichberechtigung. Da Fragen rund um die Möglichkeiten sowie Beschränkungen von Freiheit im System Schule in beiden Schuljahren präsent waren, möchte ich meine Analyse damit beginnen. Auch hinsichtlich Videospiel und Digitalisierung steht die Freiheit im Vordergrund. Bei Mobbing, Sport und Rassismus sind hingegen Fragen der (Un-)Gleichheit dominant, aber auch Solidarität spielt vielfach eine wichtige Rolle.

\section{Freiheit in der Schule: Räume der (Un-)Freiheit, aberkannte Rechte und die Notwendigkeit von Mut}

Im ersten Schuljahr wurde Freiheit in der Schule von mehreren Gruppen in Zines, Performances sowie Interventionen an der Schule, die mit Fragebogenerhebungen kombiniert waren, bearbeitet. Es zeigte sich, dass Schule Aspekte von Freiheit und Unfreiheit enthält, was in der unterschiedlichen Besetzung von konkreten Orten an der Schule im Hinblick auf Freiheit und Zwang Ausdruck fand. So wurden etwa der Ausgang der Schule, der Turnsaal und eine Couch am Gang als Symbole von Freiheit ausgemacht, wie eine von einer Schüler*innengruppe durchgeführte Befragung zeigte. ${ }^{3}$ Gerade diese konkrete Verortung machte Freiheit - als Freiheit von Zwang - erfahrbar. ${ }^{4}$

3 Befragung und Auswertung von Schüler"innen der $3 \mathrm{~A}$ und $3 \mathrm{~B}$ mithilfe selbstgestalteter semantischer Differentiale zu verschiedenen Orten an der Schule, 4.4.2019 und 6.4.2019.

4 Zur Unterscheidung eines negativen und eines positiven Freiheitsbegriffs siehe Hartmann/Offe 2011, 185f. Die Unterscheidung dieser zwei konkurrierenden Freiheitsbegriffe geht auf Isaiah Berlin (1958) zurück. Negative Freiheit bedeutet die Abwesenheit von Zwang, sfrei in diesem Sinne sind Personen mithin in dem Maße, wie sie von anderen nicht daran gehindert werden, das zu tun, was sie tun wollen. Positive Freiheit dagegen besteht in Selbstbestimmung oder Selbstverwirklichung; zwangfrei handelt demzufolge nicht schon, wer in seinem Tun von anderen nicht behindert wird, sondern erst, wer dabei 
In einem Zine aus dem ersten Projektjahr wird Schule als Ort der Aberkennung elementarer Rechte thematisiert. Festgemacht wird diese Wahrnehmung etwa an der Verweigerung des Rechts aufs Klo zu gehen oder dem mangelnden Schutz vor Mobbing. Zugleich wird die Notwendigkeit formuliert, seine Meinung zu äußern, sich zu verweigern, mutig zu sein und die Wirklichkeit zu gestalten. Hier scheint Freiheit als etwas angesprochen, für das auch selbst Verantwortung übernommen werden muss. Sichtbar wird dieser Zusammenhang von Mut (und damit Eigenverantwortung) und Freiheit beispielsweise in einem Text zur Frage »Wie zusammenleben«, den Schüler*innen aus den Wörtern eines bestehenden Gedichts zusammenstellten:

»Keine Angst haben. Sich nicht allein fühlen. Wo sich alle äußern dürfen. Ich möchte sorglos sein können. Wer hat das Kommando. Die Liebe äußern. Alleine möchte ich nicht leben. Nicht ohne Liebe sein. Recht auf ein gewaltfreies Leben. Sich etwas trauen. Sagen und dafür (Ein)stehen. Etwas nicht vorgeben. ${ }^{5}$

\section{Freiheit im Videospiel: Gestaltungswunsch und Kontrollverlust}

Der Schule stand im ersten Projektjahr ein weiterer Raum von Freiheit oder auch Unfreiheit gegenüber: der virtuelle Raum des Videospiels. Interessant ist jedoch, dass von den Schüler*innen angenommen wurde, dass dem Thema Videospiel (und übrigens auch dem Thema Sport) in einem schulischen Projekt kein Raum zugestanden würde. Hierin ist sichtbar: Das Spielen von Videogames wird als eine Möglichkeit betrachtet, die (virtuelle) Welt zu gestalten, während sonst kaum Gestaltungsmöglichkeiten gesehen werden. Gleichzeitig wird angenommen, dass die eigenen Interessen in der Schule keinen Raum bekommen würden, man also wieder in eine passive Position gedrängt wird.

In ihrem Zine befasste sich die Schüler*innengruppe dann sehr differenziert mit Dimensionen von Freiheit und Unfreiheit im Videospiel beziehungsweise durch Videospiele:

anstelle etwaiger Neigungen seinem aufgeklärten, eigentlichen oder wahren Willen folgt« (Hartmann/Offe 2011, 185).

5 Das Gedicht entstand als Koproduktion einer Gruppe von Schüler*innen beim Kick-offTermin am 16.10.2017. Die Schüler*innen bekamen Wörter aus einem Gedicht der Texterin Vlatka Frketić zur Verfügung gestellt und sollten daraus unter dem Leitmotiv Zusammenleben selbst einen Text kreieren. 
»Videospiele haben nichts mit Freiheit zu tun, manche meinen aber, dass Videospiele zur Freiheit gehören. Zur Freiheit gehören auch Regeln. Man kann auch nach Videospielen süchtig werden. Ich find Freiheit ist, wenn alle Menschen dasselbe Recht haben, niemand über sie entscheiden kann und ich fühle mich frei, wenn ich Fußball spiele. ${ }^{6}$

Diese Zeilen zeigen, dass in der Gruppe durchaus unterschiedliche Meinungen zum Thema Videogames existierten, vor allem der Zusammenhang von Freiheit und Regeln wurde hitzig diskutiert. ${ }^{7}$ Weiters wird am obigen Zitat deutlich, dass der/die Autor*in Freiheit und Gleichheit in enger Verbindung sieht: Freiheit wird als Rechtsgleichheit gefasst. Zudem thematisiert er/sie, wo beziehungsweise wann er/sie sich frei fühlt: beim Fußballspiel, also beim Sichbewegen, im Unterschied zum in der Schule ständig geforderten und auch bei Videospielen notwendigen Sitzen.

\section{Zurück in die Schule: Von der Möglichkeit oder Unmöglichkeit von Veränderungen im System Schule}

Wie bereits erwähnt, wurde der Zusammenhang Freiheit und Schule auch von den älteren Schüler*innen aufgeworfen und bearbeitet. Wiederum wurde die empfundene Unfreiheit im Schulsystem ex negativo beschrieben, verbunden mit einem Interesse an Reformen sowie gänzlich anderen Gestaltungsformen von Schule und Lernen. Die Jugendlichen fragten nach der Ursache der empfundenen Unfreiheit: "Warum ist das Schulsystem eigentlich in so einer strengen Hierarchie aufgebaut? ${ }^{8}$ Daran anknüpfend: »Kann man das Schulsystem ändern?« Und: »Wie kann der oder die Einzelne helfen, das System Schule zu verändern?«

Im Unterschied zum ersten Schuljahr zeigte sich bei den älteren Schüler*innen in der Verhandlung des Themas eine weit größere Ohnmacht,

6 Zine einer Gruppe von fünf Schüler*innen, das am 22.2.2018 gestaltet wurde (Titel: »Freiheit im Videospiel ).

7 Wenig verwunderlich, dass dies gerade Pubertierende besonders interessiert, und ebenfalls wenig verwunderlich, dass die Frage gerade in einer sich als progressiv verstehenden Schule (siehe den Beitrag von Schermann/Wallner in diesem Band), in der die Regeln des Zusammenlebens grundsätzlich als verhandelbar gelten, von den Jugendlichen aufgeworfen wird.

8 Diese und die folgenden Fragen wurden in einem Workshop am 29.3.2019 von den Schüler*innen festgehalten. Ihre Klassenkolleg*innen äußerten sich dann in Videostatements dazu. 
obwohl sie im Unterschied zu ihren jüngeren Kolleg"innen nicht mehr der Schulpflicht unterlagen, also nicht gezwungen waren, in der Schule zu sein. Aus verschiedenen Gründen wurde die Entscheidung, in eine höhere Schule zu gehen, jedoch nicht wirklich als Freiheit wahrgenommen. Denn was steht zur Wahl, wenn man noch nicht weiß, wie das Leben weitergehen soll? Die Reaktion auf die über viele Jahre gemachten Erfahrungen im System Schule war im Fall vieler am Projekt beteiligter Jugendlicher Resignation: warten, bis es zu Ende geht. Diese Haltung erwähnten mehrere Schüler"innen auch in den Gruppengesprächen. ${ }^{9}$ Wo keine positive Freiheit - also die Möglichkeit zur Selbstbestimmung und Selbstverwirklichung - gesehen wird, bleibt jedoch noch das Ergreifen der Freiheit, Nein zu sagen, sich zu verweigern ${ }^{10}$. Und von dieser Freiheit wurde und wird sehr wohl Gebrauch gemacht, wenn auch - im System Schule - grundsätzlich mit Sanktionen belegt. ${ }^{11}$

Dass die Schüler"innen den Themenbereich Freiheit und Schule sehr differenziert und auf mehreren Ebenen behandelten, wird etwa im Comic der entsprechenden Kleingruppe deutlich, in dem sich Freiheit mit Solidarität verbindet. Dieser trägt den Titel»Widerstand! Widerstand! Widerstand! Mit Mob in der Hand« (vgl. Abb. 1) und erzählt von einer fiktiven Protestaktion des Schulputzpersonals. Das Reinigungspersonal geht dabei auf die Straße und protestiert für mehr Selbstbestimmung im Beruf, unter anderem mit Slogans wie »Mehr Demokratie für die Putzpartie!« Die Schüler*innen unterstützen die Proteste. Die Geschichte geht gut aus und am Ende jubelt das Reinigungspersonal: »Wir haben es geschafft! Gerechtigkeit für alle! « ${ }^{12}$ Der Comic behandelt nicht das eigene Leiden der Schüler*innen am System Schule, sondern erzählt von anderen, häufig übersehenen Akteur"innen und deren (Un-)Freiheit: den Putzkräften, mit denen sich die Schüler"innen in ihrem Comic symbolisch solidarisierten. Der im Comic dargestellte lautstarke Protest des Putzpersonals stand gleichzeitig der in vielen Fällen resignierten Haltung der Schüler*innen gegenüber. So erwähnte etwa ein/e

9 Cruppengespräche mit Schülern der 7B, 29.3.2019 sowie 24.4.2019.

10 Zu Widerstand in Bildungskontexten siehe bspw. auch Holzer 2017.

11 Diese Widerständigkeit, »die Fähigkeit und Freiheit, aus eigenem Recht heraus `Nein ‘ sagen zu können« (Ebner von Eschenbach/Schäffter 2016, 114) kann auch als »eine wichtige Grundlage von Demokratiekompetenz (ebd.) gefasst werden.

12 Comic der Kleingruppe Freiheit und Schule, entstanden im Rahmen der Comic-Workshops am 5.3. und 6.3.2019. 
Schüler*in in einem Gruppengespräch »die Ironie, dass jeder Mensch streiken darf außer Schüler*innen « ${ }^{13}$.
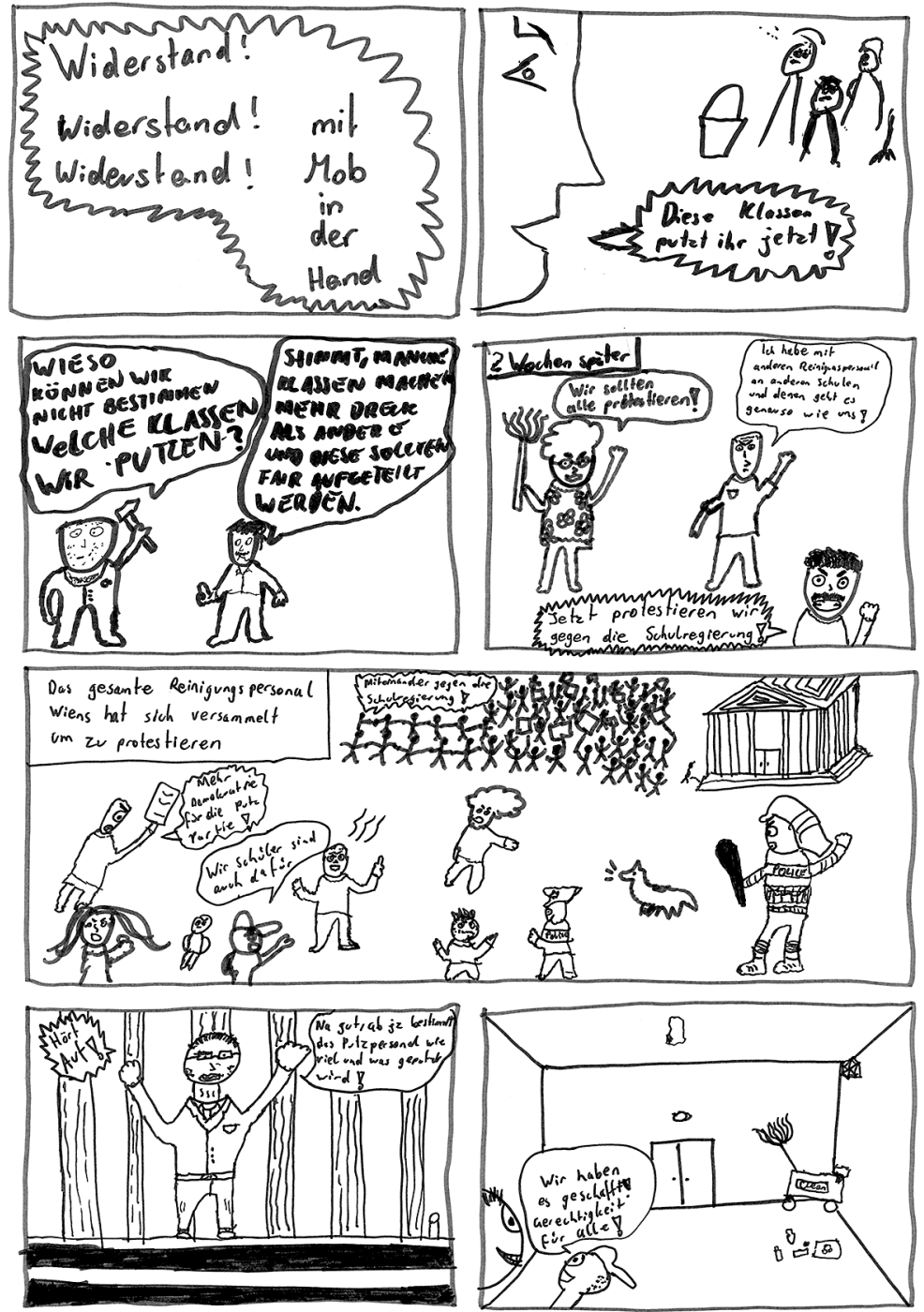

Abb. 1: »Widerstand!«, ein Comic von Schüler"innen der 7B antonkriegergasse

13 Gruppengespräche mit Schüler*innen der 7B, 29.3.2019. 


\section{Digitalisierung: Freiheiten und Freiheitsbeschränkungen}

Eine Installation mit dem Titel Die Diktatur des Likens von Anna Mayer, die sie bei einem Ausstellungsbesuch im Belvedere 21 gesehen hatten, ging einer Schüler*innengruppe im zweiten Jahr, die sich ursprünglich für das Thema Freiheit in der Kunst interessiert hatte, nicht mehr aus dem Kopf. Mayer karikiert darin Technikgläubigkeit und verschränkt Symbole aus sozialen Netzwerken, Suchmaschinen, Apps und Spielen mit religiösen Elementen. ${ }^{14}$ Daraus entspann sich im Projekt Making Democracy eine Diskussion über Chancen und Gefahren der Digitalisierung. Thema war sowohl das in vielen Peergroups gängige Mitfilmen oder Mitfotografieren beim Ausgehen als auch die Smartphone-Nutzung im Allgemeinen. Als Frage an die Mitschüler*innen zu ihrem neu definierten Forschungsthema formulierten die Jugendlichen: »Inwiefern schränkt uns Digitalisierung ein?« Eine/r der Befragten antwortete:

»Ich glaube, dass Digitalisierung uns vor allem am Arbeitsmarkt einschränken wird, weil eben so kleine Jobs wie Kassierer und so auch heutzutage schon ersetzt werden durch diese Kassenautomaten [...]. Und des Weiteren ist Kommunikation von Mund zu Mund und ebenso die Verbundenheit miteinander ... das Miteinanderreden wird auch stark eingeschränkt, was [...] sehr schade ist. Weil die Leute wissen teilweise nicht mehr wie man über ihre Gefühle redet, miteinander, weil sie alles stattdessen posten [...]. 15 $^{15}$

Dieses Zitat macht die Gedanken des/der Schüler*in rund um Chancen am Arbeitsmarkt deutlich, wirft aber auch Fragen rund um die Kommunikationsgewohnheiten der Jugendlichen auf. Diese standen dann im Zentrum der weiteren Auseinandersetzung. Zwei Plakatentwürfe, die die Jugendlichen im Rahmen der Workshops mit der Comic-Künstlerin Ka Schmitz anfertigten, setzten sich mit den Gefahren exzessiver Smartphone-Nutzung auseinander: Ein Entwurf zeigt ein Smartphone als Spritze, die sich eine offensichtlich abhängige Person intravenös setzt. Das zweite Bild zeigt eine Person, die sich hinter Gefängnismauern befindet. Die Wachtürme sind

14 Siehe Homepage von Anna Mayer: www.annameyer.at/www.annameyer.at/Der_Wert_ der_Freiheit.html\#14 (letzter Zugriff: 26.8.2019).

15 Transkript der Videoclips mit den Mini-Interviews von Schüler*innen der 7B, 29.3.2019. 
(ähnlich der Arbeit Anna Mayers) mit Symbolen sozialer Medien versehen. In den Gefängnismauern befindet sich allerdings ein Durchbruch, der mit einer Schranke versehen ist. Die Person hinter den Gefängnismauern trennt lediglich diese Schranke von der anderen Seite, auf der sich ein Regenbogen befindet, der Weg wird allerdings kameraüberwacht. Dieses Plakat ist übertitelt mit dem Aufruf: »Das Leben wartet auf dich. Mach den 1. Schrit ${ }^{11}{ }^{16}$

Die Schüler*innen stellten aber auch infrage, dass ausschließlich Jugendliche digitale Kommunikationsmittel überbordend nutzen würden. Im Vorfeld der Abschlusspräsentation befragten sie daher eine Parallelklasse zu ihrer Handynutzung. Bei der Tagung führten sie dieselbe Befragung mit den Gästen durch. Mit dem Vergleich zwischen Schüler*innen und Tagungsgästen wollten die Jugendlichen zeigen, dass es sich bei exzessiver Handynutzung um ein gesamtgesellschaftlich verbreitetes Problem handelt. Mit den Ergebnissen konnten sie pauschalisierende Vorannahmen über Jugendliche entkräften und in diesem Sinne auf eine Gleichheit von Jugendlichen und Erwachsenen verweisen.

\section{Mobbing: die soziale Situation in der Klasse und verschiedene Gleichheitsvorstellungen}

Mobbing wurde von den jüngeren Schüler*innen intensiv bearbeitet, vorwiegend auf der Ebene der eigenen Schulklasse. Die Schüler*innen hatten konkrete Zugänge und konnten die Situation in ihrer Klasse beschreiben, Erfahrungen schildern und Meinungen formulieren, wie aus den Interviews mit Mitschüler*innen, die von zwei Gruppen geführt wurden, deutlich wird. Dabei wurde Mobbing im Zusammenhang sowohl mit (fehlender) Gleichheit als auch mit einer Einschränkung der Freiheit jener, die von Mobbing betroffenen sind, angesprochen. Die Präsentation des Zines zu Mobbing vor den Mitschüler"innen sprach implizit die Tatsache an, dass alle etwas mit dem Thema zu tun hatten, was sich in auffallend angespannter Stille äußerte. Gleichzeitig hatte sich ein Mädchen aus dieser Gruppe im Vorfeld explizit an einen Jungen gerichtet, den sie als mobbend wahrnahm und ihn mit dieser ungewöhnlich offenen Kritik - durchaus produktiv - verunsichert. Die symbolische Entmachtung der Mobbenden wurde bei dieser Präsentation

16 Plakate der Kleingruppe »Digitalisierung«, entstanden im Rahmen der Comic-Workshops am 5.3. und 6.3.2019. 
durch den Verweis auf wissenschaftliche Literatur und ihre Darlegung der psychologischen Motive von Mobbing unterstützt.

Zeitgleich mit dieser reflexiven Auseinandersetzung drückte sich ein (teilweise durchaus thematisiertes und oft als Zwiespalt erfahrenes) Unvermögen oder auch ein Unwille aus, mit speziellen Befindlichkeiten und weniger ausgeprägter sozialer Angepasstheit einzelner Schüler*innen umzugehen. Dies ist in Zusammenhang mit der Situation an einer Schule zu sehen, in der die unausgesprochene Vorstellung der Gleichbehandlung aller Schüler"innen besteht, aber seitens der Lehrer"innen und damit auch seitens der Schüler*innen im Sinne der Individualisierung und der Bereitstellung gleicher Chancen doch auf die Bedürfnisse, Ausgangsbedingungen und Fähigkeiten der Einzelnen Rücksicht genommen werden muss. Individuelles Freiheitserleben und Gleichheitsanspruch werden davon insofern berührt, als es darum ginge, sich selbst in bestimmten Situationen zugunsten gleicher Chancen für alle zurückzunehmen. Das Verdecken dieses Zwiespalts beschränkt letztlich die Möglichkeiten, Demokratie zu lernen und Freiheit, Gleichheit und Solidarität zu verstehen, da es das Spannungsverhältnis zwischen einem konservativen und einem progressiven Gleichheitsbegriff verwischt: Die Wertvorstellung jedem/r dasselbe trifft auf die Wertvorstellung jedem/r, was er/sie braucht (also einer Forderung nach ungleicher Behandlung im Sinne einer Herstellung von Gleichheit).

Das Thema Mobbing brachte ein Spannungsverhältnis zwischen unterschiedlichen Gleichheitsvorstellungen an die Oberfläche, das in der Schule - angesichts schulischer Logiken von Gerechtigkeit und Leistung - schwer aufzulösen ist, erst recht nicht seitens der Schüler*innen allein. Auch wenn das Thema für die 12- bis 13-Jährigen begrifflich schwer zu fassen war, wurde es an Beispielen sehr produktiv verhandelt. So gestalteten die Schüler*innen eine Radiosendung, in der verschiedene Verläufe von Mobbing akustisch (in Geräuschkulissen) übersetzt wurden.

\section{Sport: Geschlechterverhältnisse und Gleichberechtigung}

Im zweiten Schuljahr beschäftigte sich eine Gruppe mit Geschlechterfragen beziehungsweise Fragen der Gleichberechtigung, die sie schließlich auf einen Bereich fokussierten, der für alle Teilnehmer*innen der Gruppe persönlich wichtig war: Sport. Die Jugendlichen stellten (für die Interviews ihrer Mitschüler*innen) folgende Frage: »Was soll verändert werden in Bezug auf 
mangelnde Gleichberechtigung im Sport?« Und: »Warum sind die Dinge im Sport so geregelt, wie wir sie kennen?« Bei der Beantwortung wurden die Sportförderung als auch Traditionen angesprochen, konfliktiv wurde die Frage der Geschlechtertrennung in den verschiedenen Sportarten behandelt.

Für die Abschlusspräsentation entschied sich die Kleingruppe für die Produktion eines Videoclips. Dieser zeigt eine Szene, in der mehrere Jungs Fußball spielen. Ein Mädchen kommt hinzu und möchte mitspielen, dies wird ihr allerdings mit fadenscheinigen (sexistischen) Begründungen verwehrt. Daraufhin sagt das Mädchen, dass sie wettet, besser sgaberln` zu können als die Jungs. Sie tritt dann gegen einen der Jungs an und es zeigt sich, dass sie wirklich besser spielt. Danach verlässt sie den Platz und sagt im Gehen, dass sie keine Lust mehr hat, mit ihnen zu spielen. Bei der Präsentation im Rahmen der Tagung im Volkskundemuseum Wien leiteten die Schüler*innen das Video damit ein, dass es das »Klischee der Frau « zeige. Tatsächlich zeigt das Video eher das Klischee der 'Ausnahmefrauk. Diese muss doppelt so viel leisten, um akzeptiert zu werden, sie ist also für die Lösung des Problems der (Un-)Gleichbehandlung verantwortlich.

\section{Staatsbürgerschaft und Wahlrecht: Ein- und Ausschlüsse demokratischer Systeme}

Ein Thema, das im zweiten Schuljahr früh zur Sprache kam, war jenes der Staatsbürgerschaft. Konkret interessierte die Schüler*innen-Gruppe der Zusammenhang von Staatsbürgerschaft und Wahlrecht, also Ein- und Ausschlüsse aus dem Wahlrecht und deren Konsequenzen. Bereits beim zweiten Workshop wurde die Frage formuliert: »Wer darf wo wählen und wie hängt das mit Staatsbürgerschaft zusammen?« Für die spätere Befragung ihrer Mitschüler"innen hielten sie die folgenden Interviewfragen fest: WWas ist am Wahlrecht ungerecht?«, »Wer sollte wählen dürfen?«, »Wie können wir Veränderungen des Wahlrechts hervorrufen? ${ }^{17}$

Neben Sicherheits- und Zugehörigkeitsgefühlen wurden auch Hürden in der Erlangung der Staatsbürgerschaft und mangelnde Rechte für Menschen ohne österreichische Staatsbürgerschaft thematisiert. Die Feststellung eines Unrechts mündete sodann - wie in der letzten der oben erwähnten drei Interviewfragen bereits deutlich wird - in der Frage nach Veränderungsmöglich-

17 Diese Fragen wurden beim Workshop am 29.3.2019 von den Schüler*innen formuliert. 
keiten sowie nach Hindernissen für Reformen des Wahlrechts (etwa die Bequemlichkeit jener, die sich in einer privilegierten Situation befinden ${ }^{18}$ ).

Für die öffentliche Abschlusspräsentation entschieden sich die Schüler*innen für ein Befragungssetting, das sie so ähnlich schon mit anderen Schüler*innen ihrer Schule ausprobiert hatten. Sie bereiteten Fallbeispiele vor, die folgende Eckdaten enthielten: den Vornamen, die Dauer des Aufenthalts in Österreich sowie in anderen Staaten und teils auch die Nationalität der Eltern. Sie stellten diese Fallbeispiele jeweils kurz vor und ließen die Tagungsgäste dann darüber abstimmen, ob diese fiktive Person in Österreich das Wahlrecht haben sollte oder nicht. Daran anknüpfend moderierten sie eine Publikumsdiskussion, in der erörtert wurde, warum die jeweilige Meinung vertreten wurde, was für und gegen das Wahlrecht der fiktiven Person spricht und was das für die Person bedeutet. Dabei wurden stereotype $\mathrm{Zu}$ schreibungen (entlang der fiktiven Namen) thematisiert und unterschiedliche Konzepte von Zugehörigkeit, Gleichheit und Solidarität verhandelt.

\section{Rassismus: der Wunsch gesellschaftliche Verhältnisse zu verstehen und die Schwierigkeit darüber (involviert) zu sprechen}

Rassismus, ein Themenkomplex der im ersten Schuljahr von gleich zwei Kleingruppen im Kontext von Gleichheit und Solidarität behandelt wurde, blieb im Vergleich zu den anderen Themen, obwohl sehr häufig aufgeworfen, als Problembereich weitgehend abstrakt. Ausgangspunkt war hier oft die grundlegende Ablehnung von Rassismus, die mit einem Ansiedeln von Rassismus außerhalb der eigenen Lebenszusammenhänge einherging, was durch eine weitgehende Reduktion auf die Frage der Hautfarbe verstärkt wurde. Gesellschaftliche Strukturen und Bedingungen konnten ebenso wenig herausgearbeitet werden wie Strategien gegen Rassismus, die über körperliche Selbstverteidigung hinausgehen, oder eigene Rassismen.

Hierin wird deutlich, wo die Grenzen von Forschung (aber auch Bildungsprozessen) an Schulen liegen: Bei als wenig alltagsrelevant erfahrenen und zudem hoch aufgeladenen Themen kann das Bewusstsein über die scheinbar korrekte Haltung dazu bei den Schüler*innen eine eingehende Bearbeitung derselben erschweren. Während im Projekt versucht wurde, die Interessen und Zugänge der Schüler*innen ins Zentrum zu stellen, fanden

18 Vgl. ebd. 
diese Prozesse eingebettet in das Schulsystem (und die Schulpflicht) statt. Die Schüler"innen folgten - vor allem, wenn es um das Thema Rassismus ging - einer Form sozial erwünschten Sprechens, was wohl mit dem Kontext von Schule als einem Ort, wo man das Richtige zu sagen hat, zu tun hat. Eine Thematisierung des eigenen Erlebens und der eigenen Involviertheit - auch in rassistische Strukturen - ist damit erschwert oder gänzlich verstellt. So oszillierte das Thema zwischen der Ebene zwischenstaatlicher Auseinandersetzungen und Grenzregime einerseits und der Darstellung von Rassismus als individueller, abzulehnender Einstellung andererseits.

Im zweiten Projektjahr zeigte sich Ähnliches: Die Forschungsgruppe Freiheit in der Schule stellte sich für die Abschlusspräsentation selbst unter anderem folgende Fragen, auf die sie allerdings im Unterschied zu den anderen aufgeworfenen Fragen keine Antwort gaben:

"Spielt es eine Rolle in der Schule, woher du kommst, woher deine Eltern kommen, was für eine Staatsbürgerschaft du hast? Ist das Schulsystem inklusiv und tolerant genug? Cibt's Ärger? Gibt's Konflikte? ${ }^{19}$

Die Jugendlichen deuten hier Prozesse struktureller Diskriminierung an, führen diese aber nicht weiter aus und gehen auch nicht auf die eigene Involviertheit (etwa in Bezug auf die erwähnten Konflikte) ein. Sie entziehen sich damit einer Auseinandersetzung, in der sie selbst - anders als in der Verhandlung mangelnder Freiheit in der Schule - nicht ausschließlich eine Opferrolle haben. Die Sequenz der Fragen zeigt zudem, welche Verknüpfungen die Schüler*innen ziehen: Differenz wird mit Herkunft gekoppelt, darauf folgt eine Forderung nach Inklusion und Toleranz (statt einer Veränderung der ausschließenden Norm), als letzter Schritt erfolgt die Verbindung von Differenz mit Konfliktivität. Differenz wird damit als ein potenzielles Problem gefasst.

Anders verlief die Verhandlung von Ausschlüssen aufgrund von Herkunft bei einer anderen Gruppe im zweiten Schuljahr. Bezogen auf das in Österreich vorenthaltene Wahlrecht für Menschen ohne hiesige Staatsbürgerschaft wurde klar eine Veränderung der Norm gefordert und es wurden verschiedene Positionierungen der Jugendlichen (als privilegierte Mehrheitsangehörige oder als Angehörige einer diskriminierten Minderheit) und somit die Möglichkeiten von Solidarität verhandelt.

19 Siehe den Beitrag der Forschungsgruppe Freiheit in der Schule in diesem Band. 


\section{Conclusio}

Wie sich an den Beispielen zeigt, stellen sich im Alltag der am Projekt beteiligten Jugendlichen regelmäßig Fragen von persönlicher Autonomie und deren Einschränkung, Gleichheit und Ungleichbehandlung, Solidarität und Ausschluss. Persönliche Autonomie muss in den Augen der Schüler*innen weniger gegenüber den Gleichaltrigen als vielmehr gegenüber der Schule verteidigt werden. Während die Schule deutlich Zwang verkörpert, steht die Klasse stärker für Gemeinschaft. Insofern ist das Ziel eher, gemeinsam frei zu sein, eine Situation herzustellen, »wo sich alle äußern dürfen«, wie Schüler*innen beim Kick-off-Termin im ersten Schuljahr ihre Vorstellung von $\mathrm{Zu}$ sammenleben fassten. Aber es gibt auch eine andere Seite: Durch die Wahrnehmung von Andersheit kommt es, wie am Beispiel Mobbing gezeigt wurde, zur Aberkennung von Solidarität und (auch in den Augen der Schüler*innen) zu einem Verlust an Freiheit. Der Anspruch, eine Gemeinschaft zu sein, ist gerade angesichts dessen, dass es sich bei einer Schulklasse um keine gewählte Gemeinschaft handelt - unterschiedlich ausgeprägt. Entsprechend ist der Anspruch, auf individuelle Eigenheiten und Bedürfnisse Rücksicht zu nehmen, unterschiedlich stark - zumal hierzu ja die Logiken von Leistung und Anpassung, die aus gesellschaftlichen Normen herrühren und auf die Schule miteinwirken, im Widerspruch stehen.

In beiden Schuljahren wurde deutlich, dass Fragen rund um demokratische Werte vorwiegend ex negativo gestellt werden: Die Thematisierung von Freiheit, Gleichheit und Solidarität erfolgte in den meisten Fällen über deren Fehlen oder Mangel. Dies zeigte sich in Themenstellungen wie Mobbing, Geschlechterungleichbehandlung, schulischer Zwang, elterliche Verbote, Rassismus oder Ausschlüsse aus dem demokratischen System. Die Jugendlichen im zweiten Schuljahr thematisierten zwar Werte ex negativo (Rechte über Unrecht, Freiheit über Zwang), sahen sich selbst jedoch stärker eingebunden in komplexe Gefüge, in denen nicht nur das eigene Recht, die eigene Freiheit zählen, sondern unterschiedliche gesellschaftliche Positionen beachtet werden müssen: zum Beispiel, wenn die Folgen der Digitalisierung für den Arbeitsmarkt auch im Verkauf eingebracht wurden.

Auffallend war vor allem im ersten Schuljahr die Schwierigkeit, sich der Idee der Solidarität anzunähern. Nicht nur war der Begriff selbst kaum bekannt, sondern es fehlte, bei aller Klarheit in Bezug auf die Bedeutung von Zusammenhalt, oft an einer Vorstellung von Zusammenhalt mit Menschen, 
zu denen kein klares Naheverhältnis besteht. Die Tatsache, dass der Begriff der Solidarität nicht nur den Aspekt der Solidarität unter Gleichen umfasst, sondern ebenso jenen der Solidarität unter Unvertrauten, und es hier auch um ein Handeln ohne Gegenleistung, aber im Sinne des großen Ganzen geht, war für die Jugendlichen zum Teil wenig zugänglich und irritierte sie. Mehr als bei den anderen Themen beinhaltete das Sprechen der jüngeren Schüler*innen über Solidarität häufig Allgemeinplätze. Solidarität wurde teils mit Spenden gleichgesetzt und paternalistische Formen des Helfens standen im Vordergrund. Schwierig war auch das Sprechen über die eigene Involvierung in Ungleichheit produzierende gesellschaftliche Verhältnisse wie Rassismus. Die Thematisierung ihrer eigenen Erfahrungen von Unfreiheit im Schulsystem fiel den Schüler"innen hingegen leichter zu artikulieren.

Insgesamt stellten wir fest, dass der Umgang mit großen Begriffen wie Demokratie, Freiheit, Gleichheit und Solidarität die Schüler"innen vor große Herausforderungen stellte. Denn diese Begriffe sind als »essentially contested concepts «(Gallie 1956) letztlich mehrdeutig - die Jugendlichen müssen sie erst mit konkreten Bedeutungen füllen, um sie sich anzueignen, und damit eine Theoretisierungsleistung erbringen. Die Annäherung an das Politische gelang über konkrete Erfahrungen und alltägliche Handlungszusammenhänge und nicht über abstrakte Begriffe, ein Aspekt, der auch in der politischen Bildung berücksichtigt werden sollte.

\section{Literatur}

Ebner von Eschenbach, Malte/Schäffter, Ortfried (2016). Epistemische Widerständigkeit in der Politischen Bildung. Verantwortungsvoller Umgang mit Differenzen als Demokratiekompetenz, in: Reheis, Fritz/Denzler, Stefan/Görtler, Michael/Waas, Johan (Hg.): Kompetenz zum Widerstand. Eine Aufgabe für die politische Bildung, Schwalbach: Wochenschau, 112121.

Gallie, Walter Bryce (1956). Essentially Contested Concepts, in: Proceedings of the Aristotelian Society, Vol. 56, 167-198. https://doi.org/10.1093/aristo telian/56.1.167.

Garnitschnig, Ines (2019). Sehen verlernen mit kritischer Kunstvermittlung und partizipativer qualitativer Forschung, in: Gottuck, Susanne/Grünheid, Irina/Mecheril, Paul/Wolter, Jan (Hg.): Sehen lernen und verlernen. 
Perspektiven pädagogischer Professionalisierung, Wiesbaden: Springer VS, 263-286. https://doi.org/10.1007/978-3-658-19496-3_12.

Hartmann, Martin/Offe, Claus (2011). Politische Theorie und Politische Philosophie. Ein Handbuch, München: C. H. Beck.

Holzer, Daniela (2017). Weiterbildungswiderstand. Eine kritische Theorie der Verweigerung, Bielefeld: transcript. https://doi.org/10.14361/9783839 439586.

Rajal, Elke/Garnitschnig, Ines/Marchart, Oliver (2019). Demokratie im Alltag. Aushandlungen von Freiheit, Gleichheit und Solidarität unter Jugendlichen im Rahmen des Forschungsprojekts "Making Democracy«, in: SWS-Rundschau, Heft 2/2019, 59. Jahrgang, 181-199.

Zobl, Elke (2011). »A Kind of Punk Rock `Teaching Machine«. Queer-feministische Zines im Kunstunterricht, in: Art Education Research, Nr. 3, abrufbar unter: https://blog.zhdk.ch/iaejournal/files/2012/02/eJournal-AE R-no-3_Zobl.pdf (letzter Zugriff: 9.8.2019). 


\title{
Das Projekt Making Democracy als Gegenstand demokratischer Forderungen und als Raum für deren Artikulation
}

Nora Landkammer

\begin{abstract}
Im Projekt Making Democracy wurden Kernbegriffe der Demokratie - Freiheit, Gleichheit und Solidarität - mit Jugendlichen an einer Wiener Schule in einem partizipatorischen Forschungsprojekt aufihre Bedeutung im Alltag untersucht. Das Projekt wurde im zweiten Jahr selbst zum Anlass für Konflikte zum Thema Demokratie in Forschung und Schule. Wie handeln Sozialwissenschaftler*innen, Kunstvermittler*innen, Lehrer*innen und Schüler*innen Demokratie aus? Der Beitrag arbeitet heraus, dass Making Democracy zum Gegenstand demokratischer Forderungen wurde, und zugleich Raum für deren Artikulation bot. Der Prozess wird daraufhin untersucht, welche Verständnisse von Demokratie sich darin zeigten und wie die Beteiligten diese aushandelten. Dabei werden Kernthemen identifiziert, die Aufmerksamkeit benötigen, wenn das »Machen « von Demokratie in partizipatorischen Projekten im Fokus stehen soll.
\end{abstract}

\section{Wir wurden ja nicht gefragt}

Ich fokussiere in diesem Beitrag auf das zweite Jahr von Making Democracy, mit dem ich Teil des Projektteams wurde. ${ }^{1}$ Als Ausgangspunkt dient die Tatsache, dass das Projekt Making Democracy selbst zum Gegenstand demokratischer Forderungen wurde.

1 Siehe für einen Überblick über das Projekt und die Auswertung beider Schuljahre den Beitrag von Elke Rajal in diesem Band. 


\section{S: »Das ist ja das Hauptproblem.« \\ $\mathrm{V}:$ »Was?《}

S: »Dass es um Demokratie geht und wir nicht gefragt wurden.« (FTB NL, 19.2.2019) $)^{2}$

Dieser Dialog zwischen einer der Vermittlerinnen und Forschenden im Projekt und einem Schüler rührt zunächst von einem organisatorischen Problem her: Eine 6. Klasse wurde vor den Sommerferien gefragt, ob sie ein Forschungsprojekt machen wollen. Nach dem Sommer, als alles organisiert war, saßen in dieser 7. Klasse dann großteils Schüler*innen, die bei der Entscheidung nicht dabei waren - weil sie eine Klasse wiederholten, aus anderen Klassen zusammengelegt wurden, oder die Schule gewechselt hatten.

Über das Organisatorische hinaus spricht der Dialog das Verhältnis zwischen Regelschulsystem und partizipativer Forschung an, wirft also methodologische und forschungsethische Fragen auf. Partizipative Forschung als Paradigma, mit einer Vielzahl unterschiedlicher Modelle und Traditionen, ist dadurch definiert, dass die Akteur*innen in dem Feld, das untersucht wird, auch diejenigen sind, die selbst als Forschende aktiv werden. In unserem Fall hieß das, dass die Schüler*innen ihre eigenen Fragen entwickelten, denen sie mit unterschiedlichen Methoden und in diversen Medien nachgingen; während wir als Wissenschaftler"innen und Vermittler"innen in der Schule ebenso als Akteur*innen und Mitbeforschte Erkenntnisse über das Aushandeln von Demokratie anstrebten. ${ }^{3}$ Freiwilligkeit gilt als Grundprinzip partizipatorischer Forschungsansätze, ist aber bei der Zusammenarbeit mit Co-Forscher*innen in stark regulierten institutionellen Settings wie Schule, Krankenhaus oder Gefängnis oft nur eine relative. Es stellt sich daher die Frage: Wie ist die Selbstbestimmung der Co-Forscher*innen in einem meist nicht selbstbestimmten institutionellen Setting wie dem Schulsystem zu ermöglichen? Für das Sparkling-Science-Projekt traf das Team die Entscheidung, mit Schüler*innen im Regelunterricht zu arbeiten, um die Teilnahme

2 Forschungstagebuch Nora Landkammer (hier und im Folgenden: FTB NL), mitprotokollierte Cesprächssequenz.

3 Es handelte sich also um ein Ebenen-Modell, das die eigenen Fragestellungen und Erkenntnisprozesse der Schüler*innen mit der Fragestellung des Projektteams kombinierte. Schüler*innen sind dabei Forschende und zugleich (ebenso wie das Projektteam selbst) Beforschte und Datenquellen. Zu einer Systematik unterschiedlicher Rollen der Teilnehmerinnen in Aktionsforschungsprojekten siehe Bragg/Fielding (2005, 207ff.). 
nicht auf Schüler*innen zu beschränken, die sich für ein Freifach anmelden, was häufig schulischen Erfolg und Habitus aus dem Elternhaus widerspiegelt und schlussendlich Ausschlüsse reproduziert. Im Gegenzug ist die Freiwilligkeit in einem solchen Setting per se ein »wählender Zwang« (Schüler, GS 29.3.19), ${ }^{4}$ zwischen normalem Unterricht oder eben Projektunterricht. Auf methodischer und forschungsethischer Ebene ist diese Spannung für partizipatorische Forschung und Aktionsforschung in der Schule unter anderem in Sparkling-Science-Projekten bereits reflektiert worden. So fragen sich Veronika Wöhrer und das Forschungsteam der Projekte Tricks of the Trade und Grenzgänge, die aus den Erfahrungen in Sparkling-Science-Projekten ein Methodenhandbuch erarbeitet haben, inwiefern in einem solchen Setting tatsächlich von gemeinsamer Forschung, also einem geteilten Interesse gesprochen werden kann (Wöhrer et al. 2017, 21f.). Die forschungsethischen Überlegungen sind oft aus einer nachträglichen, zweifelnden Sicht der Forscher"innen formuliert. Habe ich die Entscheidungen der Co-Forschenden zu viel beeinflusst? Wie partizipativ war das Projekt? $\mathrm{Zu}$ dieser Nachträglichkeit der Reflexion kam es in unserem Projekt gar nicht, weil die Schüler*innen die Rahmenbedingungen unserer Zusammenarbeit wiederholt und beständig zum Thema machten und infrage stellten. So führten wir ein Jahr lang Diskussionen nicht nur über Demokratie im abstrakten und konkreten Sinne, sondern über die Demokratie im Projekt selbst.

Das ist schließlich nicht nur methodisch von Interesse, sondern ganz wesentlich für die Forschungsfrage nach der Aushandlung von Demokratie im Alltag. Es ist wichtig, dass nicht nur das Handeln der Jugendlichen der Forschungsgegenstand ist, sondern auch unser Handeln als Forschungs-/Vermittlungsteam, dass wir also uns selbst als Akteur*innen in den Blick bekommen. Die Frage lautet: Wie handeln Schüler*innen, Lehrer*innen, Vermittler*innen und Sozialwissenschaftler*innen Demokratie aus?

Ich möchte hier auf die Prozesse mit den Schüler*innen fokussieren, die sich am stärksten in die Debatten über das Projekt und seine Rahmenbedingungen einbrachten. Mitzudenken ist, dass dadurch andere Schüler*innen in den Hintergrund treten und die Klasse leicht als homogenes Kollektiv erscheint, das sie nicht war.

4 Gruppeninterview mit Schüler*innen (hier und im Folgenden: CS). Die Gruppeninterviews wurden mit Audioaufnahmen dokumentiert und transkribiert. Die zitierten Inteviewpassagen wurden redigiert. 
Das eingangs erwähnte »Wir wurden ja nicht gefragt« ist in einer umfangreicheren Reihe zu lesen, der Wahrnehmung, keine Mitbestimmungsmöglichkeiten $\mathrm{zu}$ haben und nicht angehört zu werden, in der Schule und darüber hinaus. In meinen Forschungstagebucheinträgen zu den Diskussionen mit den Schüler*innen und den Gruppeninterviews zu ihren Wahrnehmungen von Mitbestimmung in der Schule häufen sich Aussagen wie diese:

\begin{abstract}
»Aber wir wurden nie gefragt, [...] was uns eigentlich interessiert.«(CS 29.3.2019) »ch meine, wir wurden ja noch nicht mal gefragt, in was wir maturieren wollen.«(CS 29.3.2019)

»Wir haben halt inhaltlich keine Chance was zu sagen, weil wenn wir was sagen, dann ist es ja wurscht.«(CS 24.4.2019)

»Und in Englisch können wir grade mal entscheiden, was für Bücher wir lesen müssen.«(CS 29.3.2019)

»Und dann haben sie es gemacht, ohne überhaupt uns zu fragen. Oder überhaupt. Einfach so. Ohne abzustimmen. Ja oder bei der neuen Matura. War genau das gleiche. Das haben sie einfach gemacht, ohne dass es eine Abstimmung gab.«(CS 24.4.2019)
\end{abstract}

Entsprechend verblüfft und entrüstet war die Reaktion einer Schüler*innengruppe, als sie auf DemokratieWEBstatt, einer Website des österreichischen Parlaments für politische Bildung, auf diesen Text stießen:

»In der Schule erlebst du täglich Demokratie hautnah, denn die Schulen in Österreich sind demokratisch organisiert. Das nennt man Schulgemeinschaft. In einer Schulgemeinschaft arbeiten LehrerInnen, Erziehungsberechtigte und SchülerInnen demokratisch zusammen. Das bedeutet, dass zum Beispiel nicht die Direktorin oder der Direktor >das Sagen über alles hat, und was sie oder er sagt, muss genauso geschehen. Demokratie bedeutet `Herrschaft des Volkes < und >das Volk« besteht in diesem Fall aus allen Beteiligten in der Schule: den LehrerInnen, den Eltern und natürlich den SchülerInnen. $\ll^{5}$

5 DemokratieWEBstatt - Ein Angebot des österreichischen Parlaments zur politischen Bildung, https://www.demokratiewebstatt.at/thema/schule-und-demokratie/ (letzter Zugriff: 29.7.2019). 
Auch wenn den Schüler*innen der Schulgemeinschaftsausschuss bekannt war, hätte es kaum eine Beschreibung geben können, die sich weniger mit ihrer Wahrnehmung deckte. Sie bezeichneten den Text als Lüge, und stellten die Vermutung an, er sei wahrscheinlich von jemandem verfasst, die/der niemals in einer Schule war.

\section{Making democracy als Kristallisationspunkt für Forderungen nach Mitbestimmung}

Ich möchte folgende, miteinander verbundene Thesen formulieren:

- Anhand des Projekts wurden fehlende Mitbestimmung in einem breiteren Rahmen (in der Schule) und die Frage nach Strukturen und Realität von als demokratisch ausgewiesenen Prozessen zum Thema.

- Die Tatsache, dass das Projekt mit Begriffen wie »Demokratie«, »Gleichheit « und »Freiheit« antrat und offen den Anspruch erhob, partizipativ $\mathrm{zu}$ sein, machte es zum idealen Kristallisationspunkt für die Verhandlung von Unfreiheit und Ungerechtigkeit, die die Schüler*innen in ihrem Alltag beschäftigen.

- Durch das Fragen nach Demokratie in einem als undemokratisch identifizierten Kontext schwankte das Projekt in seiner Funktion hin und her: als Gegenstand demokratischer Forderungen und als Raum für ihre Artikulation.

Dieser Wechsel in der Wahrnehmung des Projekts prägte die Zusammenarbeit: engagierte Diskussionen, gefolgt von einer plötzlichen Distanzierung. Das oft unvermittelte Hin- und Herwechseln zwischen Interesse und Ablehnung, Diskussionsfortschritten und dem Zurückkehren zur Verhandlung über die investierte Zeit und die Termine durchzog viele Schultage. Die Schüler*innen kritisierten das Projekt als aufoktroyiert, stellten infrage, inwiefern die Diskussionen mit dem Projektteam tatsächlich demokratisch abliefen, und stellten den Zeitaufwand sowohl ihrem Bedürfnis nach mehr Freizeit als auch der Notwendigkeit für die Schularbeitsfächer zu lernen entgegen. Diese Kritik war zugleich Kristallisationspunkt für weitergehendes Hinterfragen des Bildungssystems und der schulischen Strukturen. Der Prozess war selbst Gegenstand der Forderung nach mehr Demokratie, ein 
Exempel der Probleme, die die Jugendlichen in der Institution Schule sahen, und wurde zuweilen gerade deshalb ein Raum, um diese Problematiken zu ergründen und zu diskutieren.

\section{Solidarität und Handlungsmacht in Schul- und Projektkritik}

Das »Wir wurden ja nicht gefragt" passt zunächst in das Bild der Politikverdrossenheit, das Studien zu Jugend und Politik zeichnen (siehe den Beitrag von Elke Rajal in diesem Band) und zur Gesellschaftsdiagnose der Postdemokratie (Crouch 2008; Gerdes/Bittlingmayer 2016): Das Individuum steht einem übermächtigen System gegenüber, an dem es gefühlt keinen Anteil hat; die eigene Handlungsmacht wird als gering eingeschätzt (siehe Schüler*innen-Zitat: »... wenn wir was sagen, ist es ja wurscht«, GS 24.4.2019), im Gegensatz zum Impetus von Making Democracy, zum Mitgestalten und Auseinandersetzen anzuregen.

Im Handeln der Schüler*innen im und gegen das Projekt zeigte sich aber keineswegs die geringe Agency und die passive Rolle, die »Politikverdrossenheit« unterstellt. Im Gegenteil forderten die Schüler*innen anhand des Projekts eine Aushandlung vehement ein. Ihr Blick war auf Autoritäten gerichtet, sie schrieben der Figur des Direktors viel Macht zu und sahen sich selbst am untersten Ende der Hierarchie. Sie sahen sich aber auch in der Rolle, an diese Autorität mit ihren Anliegen heranzutreten. So bildeten die Schüler*innen eine Kommission, die vor dem Direktor einige Kritiken und Forderungen der Schüler*innen vorbrachte.

Einer in der Projektplanung formulierten These widersprach das Agieren der Schüler*innen ebenfalls. Im Antrag für das Projekt wurde, in Verbindung mit der Diagnose der Postdemokratie, die Frage gestellt, inwiefern sich die neoliberale Aufwertung von individueller Autonomie gegenüber Werten von Gleichheit und Solidarität (Mouffe 2008) im Projekt mit den Jugendlichen abbilden würde. Das Handeln der Schüler*innen bei unseren Aushandlungen über das Projekt und seine Rahmenbedingungen bestätigte diese Tendenz nicht. Zwar ging es bei der Auseinandersetzung mit den Vorgaben und Zwängen in der Schule um individuelle Autonomie, das Recht auf mehr Selbstbestimmung in der eigenen Bildung, aber das Vorgehen der Schüler*innen war dabei stark von einer Identifikation als Kollektiv geprägt. Die Zwangsgemeinschaft einer Schulklasse zeigte sich auch als 
Solidargemeinschaft, und das obwohl die Klasse neu zusammengewürfelt war und sich zu Beginn des Schuljahres noch wenig kannte. Zum Beispiel wurden Forderungen zum Projekt als Kollektiv formuliert, und den Vermittler"innen war oft nicht klar, wer was eingebracht hatte; Schüler*innen waren bereit, Inhalte zu vertreten, die sie selbst gar nicht teilten. Sie entschieden sich auch bei der Abschlusstagung und bei Veröffentlichungen als Kollektiv 7B aufzutreten und keine individuellen Namen zu nennen.

Und schließlich widerspricht der Sicht der Politikverdrossenheit, dass es um konkrete Kritiken an den Strukturen ging, die Mitgestaltung entgegenstehen. Aus verschiedenen Gesprächen zusammengefasst bemängelten die Schüler*innen:

- dass es nur zu wenigen Aspekten von Schule überhaupt Mitbestimmungsmöglichkeiten gibt;

- dass »Entscheidungen« oft nur zwischen eng vorgegebenen Optionen stattfinden, ohne tatsächliche Alternativen;

- informelle Hierarchien, die in der offiziellen Vertretungsstruktur dazu führen, dass Schüler"innenanliegen nicht wahrgenommen werden;

- eine Einschränkung von selbstbestimmtem Lernen und Unterrichten, nicht nur für die Schüler*innen, sondern auch für die Lehrer*innen, durch die Zentralmatura ${ }^{6}$;

- die Verbindung von Partizipation mit Hierarchie und Sanktion, in der »Meinungen« geäußert werden sollen, aber nicht erwünschte Meinungen sanktioniert werden.

Das Video zum Thema Schule, das eine Gruppe von Schüler*innen produzierte (siehe den Beitrag der Forschungsgruppe Freiheit in der Schule in diesem Band) führt einige dieser Punkte aus. Dass die Kritik anhand von Making Democracy entwickelt wurde und das Projekt sowohl Gegenstand als auch Rahmen für die Ausarbeitung dieser Kritiken war, stellte das Projektteam vor eine schwierige Entscheidung: entweder die Konsequenz aus den Forderungen zu ziehen und das Projekt abzubrechen, oder anzuerkennen, dass hier Demokratie gefordert und ausgehandelt wird, aber zu akzeptieren, dass wir dabei zumindest zeitweise die Gegenspieler*innen sind. Wir entschieden uns für die ungemütliche zweite Option. Innerhalb des offensichtlichen

6 Teilstandardisierte kompetenzorientierte Reifeprüfung, in Österreich 2014/15 eingeführt. 
Widerspruchs zwischen der Vorstellung von Selbstbestimmtheit in der eigenen Forschung der Schüler*innen und der Unfreiwilligkeit der Rahmenbedingungen war dieses Gegenspiel von einer Reihe weiterer Paradoxien durchzogen. An unserem Handeln als Projektteam und dem der Schüler*innen lassen sich drei Kernthemen festmachen, die es erschwerten, dass Making Democracy von der Kritik an der Pflichtübung zum Handlungsraum für Veränderungen des Systems Schule werden konnte. Diese drei Paradoxien sind mitzudenken, wenn Demokratie und Alltag im Schulkontext zur Verhandlung gestellt werden.

\section{Paradoxie 1: Die Bedeutung von Alltagspraxen und die Macht der Struktur}

Ein für die Konzeption des Projekts zentraler Begriff war der des Aushandelns. Dass Freiheit, Gleichheit und Solidarität nicht als fixe Größen existieren, sondern das Resultat von Aushandlungsprozessen sind, war Ausgangspunkt und Gegenstand des Projektdesigns. Dabei stand im Mittelpunkt, dass diese Aushandlung, die mit Kontingenz assoziiert ist - also der Möglichkeit, dass alles immer auch anders hätte kommen können - nicht nur in institutionalisierten politischen Strukturen stattfindet, sondern auch im Alltag. Wie ein/e Kolleg*in in der Abschlussreflexion ausführte, sollte es darum gehen, über die Projektarbeit mit den Schüler*innen »auf diese verschiedenen Ebenen [zu] stoßen von Machtverhältnissen, Ein- und Ausschlüssen, Forderungen, Wünschen«, vom »Konflikt mit der/dem Schüler*in daneben bis zur Gesellschaft, die dann rauskommt (GT 23.5.2019). ${ }^{7}$ Es ginge darum, so die/ der Kolleg*in weiter über die Zielvorstellungen, »dass man sich selber, also jede die mitmacht, ins Verhältnis zur Welt setzt. Da verhandeln wir Bilder, Strategien, Möglichkeiten ... dass da so ein Begreifen stattfindet, dass man auf was draufkommen kann« (GT 23.5.2019). Neben dem Impuls einer emanzipatorischen Pädagogik zeigen die Zielvorstellungen für den Prozess die Prägung der Cultural Studies im Verständnis von Alltag und Machtverhältnissen. Die Auseinandersetzung mit dem Decodieren von Bildern und dem Verständnis von Begriffen geht davon aus, dass gesellschaftliche Machtverhältnisse nicht nur an den offensichtlichen Orten zu finden sind, sondern

7 Gruppeninterview mit dem Team (hier und im Folgenden: CT). 
Alltagshandeln, Bildwelten und Selbstverständnisse durchziehen. Hegemoniale Verhältnisse beruhen auf den geteilten Selbstverständnissen und diese können auch infrage gestellt werden (vgl. den Beitrag von Ingo Pohn-Lauggas in diesem Band). "In diesem Zusammenhang spielt der theoretisch avancierte Kulturbegriff der Cultural Studies eine große Rolle, der Kultur im weitesten Sinne als aktiven Prozess der Bedeutungsgebung und Aushandlung [...] versteht. Genau diese Sichtweise des Kulturellen ist im pädagogischen Feld eher selten anzutreffen." (Sauter 2006, 113) Sie ist ein Kernpfeiler kritischer Kunstvermittlung. Die Umdeutung, die Taktiken der Bedeutungsbildung sind demnach Orte des Widerstands und der Transformation, auch von Subjektpositionen, die im formalen Sinn mit wenig Macht ausgestattet sind.

Der Aushandlung von Verhältnissen auf allen diesen Ebenen schob sich die Aushandlung eines bestimmten, klar sichtbaren Machtverhältnisses vor: zwischen der Schulstruktur, in die wir das Projekt eingebettet hatten, und den Schüler"innen, die dieser Struktur unterworfen waren, wenn sie im System bleiben und Matura machen wollten. Dieses war ein von Autorität und Regeln geprägtes Verhältnis, in dem das Projektteam strukturell auf der machtvollen Seite stand. Solange, so könnte die Situation erweitert werden, das pädagogische Verhältnis nicht in seiner Machtstruktur ausverhandelt wird, kann die Betonung der weniger evidenten Dynamiken von Macht und Widerstand in Alltagspraxen nur als Ablenkungsmanöver wirken, das der Verschleierung der eigenen Position dient. Dieser Prozess war jedoch durchaus mit Verschiebungen im Verständnis der Verhältnisse verbunden. Während zunächst von den Schüler*innen Hierarchie, gesteuert von einer zentralen Autorität, hervorgehoben wurde, betonte ein Schüler im Video zum Thema Schule auf die Frage: »Wieso dürfen alte Säcke bestimmen, was wir lernen, obwohl sie seit Jahren keine Schule mehr betreten haben?« »Weil wir es erlauben! (Video, siehe den Beitrag der Forschungsgruppe Freiheit in der Schule in diesem Band). Das Statement kehrt hervor, wie sehr die Schulstruktur vom Einverständnis, vom Mitwirken der Schüler*innen getragen wird - also auf einem hegemonialen Verhältnis beruht, das auf einer gewissen Form der Zustimmung basiert (vgl. den Beitrag von Ingo Pohn-Lauggas in diesem Band). Das Projekt zeigte so besonders deutlich die paradoxe Aufgabe kritischer Vermittler*innen, Forscher"innen und Pädagog*innen auf, gerade die Widerstände, die sich gegen sie selbst richten, auch inhaltlich ernst zu nehmen, denn da kann das tatsächliche Begreifen davon stattfinden, dass die Dinge »komplizierter sind, als sie zunächst wirken« (GT 23.5.2019). 


\section{Paradoxie 2: Im neoliberalen Kontext im eigenen Interesse handeln}

Paradoxes Handeln durchzog das Projekt, indem wir einerseits das Setting und die Inhalte beständig zur Verhandlung stellten, andererseits immer wieder auf der strukturellen Ebene die Mechanismen bedienten, die doch Zentrum der Kritik der Schüler*innen waren. Ein Kernthema ist hier das Verhältnis des Projekts zu Zwang und Eigeninteresse im neoliberalen Kontext. Unter neoliberalen Bedingungen wird im Bildungssystem Eigeninteresse und Selbstverwirklichung betont. Diese stehen jedoch im Kontext einer Regierungstechnik, in der "Selbstverantwortung und Leistungsbereitschaft zwar gesellschaftlich gefordert werden, die einzige Möglichkeit, wirklich etwas zu bewirken, für Viele aber vor allem darin besteht, den eigenen Körper, die eigenen Emotionen oder die eigene Einstellung zu ändern« (Duttweiler 2016, o. S.). Die Einzelnen werden, so lässt sich die Dynamik der Arbeit am Selbst für Anpassung und Verwertbarkeit zusammenfassen, "zur Selbstoptimierung ermächtigt« (ebd.).

In diesem Kontext betrachteten wir erst im Nachhinein unsere Versuche, die Schüler*innen von ihrem Nutzen an dem Projekt zu überzeugen, wie beispielsweise das Anbieten eines Zertifikats mit dem Logo der Universität. Gesten wie diese reflektierten wenig mit, dass das scheinbare Eigeninteresse an der Leistung über ein vages Versprechen von Pluspunkten im Lebenslauf von den Jugendlichen längst enttarnt war, insbesondere von Jugendlichen, die mit ihrer Individualität und ihren eigenen Interessen im Schulsystem bereits angeeckt und gescheitert sind. Viele Schüler*innen lehnten das typisch neoliberale Verschwimmen von eigen- und fremdbestimmtem Handeln ab. Ihr Beharren darauf, zu tun, was verpflichtend ist oder was sie wollen (und nicht beides zugleich), war eine widerständige Haltung gegen diese neoliberale Grenzverwischung. Am deutlichsten äußerte sich die Paradoxie in der von uns in einer Nachbesprechung nach einem Schultag entwickelten Fantasie, die Schüler"innen würden ihren Widerstand gegen Making Democracy selbst zum Projekt machen und eine Performance dazu gestalten - ein Wunsch, den wir sogleich als Instrumentalisierung der Kritik der Schüler*innen identifizierten und der dennoch wahr wurde, als die Schüler*innen bei der Tagung ihr Video zur Kritik am Schulsystem und am Projekt zeigten. Das Video zeigt diesen Widerspruch, dass der von den Schüler*innen formulierte Widerstand zugleich als Erfüllung der strukturellen 
Anforderungen der Schule, des Projekts und des Fördergebers verwertet werden kann.

In partizipativer Forschung und kritischer Vermittlung, so auch in unserem Antrag, scheint das Ausgehen von den eigenen Interessen der Teilnehmer*innen häufig eine zentrale, gewissermaßen selbstevidente Kategorie, die als Garant für die Ethik der Partizipation steht. Dass diese im Kontext anderer Interessen stehen, die in einem solchen Projekt wirken, durch Institutionen, Förderstrukturen und dadurch ermöglichte Arbeitsplätze, benötigt ebenso Reflexion wie die Doppelbödigkeit des forschenden, aktiv das eigene Lernen gestaltenden Subjekts im Neoliberalismus.

\section{Paradoxie 3: Meinungsfreiheit und Hegemoniekritik}

In partizipativen Forschungsprojekten im Schulkontext befinden sich die Forscher*innen/Vermittler*innen in einer mehrdeutigen Rolle. Ihre Projektleitung greift zum einen Strukturen der Schule auf und sie übernehmen temporär Verantwortungen, die der Lehrperson obliegen. Zum anderen soll, wenn die Schüler*inneninteressen im Vordergrund stehen, die Projektleitung die Rolle der Fürsprecher*in und Koordinator*in der von den Schüler*innen definierten Ziele übernehmen. Die Forscher"innen handeln in einem Spannungsfeld zwischen diesen Rollen (Bland/Atweh 2007, 345). Dieses spitzt sich zu, wenn wie in unserem Fall Demokratie selbst das Projektthema ist.

Es wurde deutlich, dass die Schüler*innen über ein ausgeprägtes Sensorium für Pseudopartizipation und die Fallen von Meinungsfreiheit in Machtverhältnissen verfügten. Diskussion wäre noch nicht per se demokratisch, argumentierte beispielsweise ein Schüler, sondern nur dann, »wenn es Konsequenzen hat" (FTB NL, 6.3.2019). Die Erfahrung, nach der eigenen Meinung gefragt zu werden, wird von Schüler*innen als tückisch beschrieben: Sie würden schon in der Schule manchmal nach ihrer Meinung gefragt, aber wenn es die falsche wäre, bekäme man erst recht Probleme (GS 24.4.2019). Was demnach bei den Aushandlungen bei Making Democracy permanent auf dem Spiel stand, war, ob und wie die Ansichten und Meinungen tatsächlich wahrgenommen wurden. Damit verknüpft war der Zweifel, ob das Projektteam wirklich als Aushandlungspartner auf gleicher Ebene stand oder die im Schulsystem etablierte Autorität über das Wissen nutzte. 
Konkret wurde für uns die Problematik, wenn sich in Meinungen der Schüler*innen normierende Vorstellungen und gesellschaftliche Ausschlüsse entlang von Geschlecht, sexueller Orientierung oder Herkunft spiegelten. Der Anspruch des Projektteams, gerade hier Gegenpositionen sichtbar zu machen, passte sich für Schüler*innen schnell in die von ihnen kritisierte Struktur der selektiven Meinungsfreiheit in der Schule ein. Das Verhältnis Schüler*innen - Lehrpersonen/Vermittler*innen/Forscher*innen überkreuzt sich hier mit Machtverhältnissen zwischen hegemonialen und marginalisierten Positionen. Wie können Räume im Sinne einer konfliktorientierten Demokratie geschaffen werden, wenn Dissens selbst als Machtausübung interpretiert wird? Die Herausforderung, Räume zu öffnen, in denen alle Positionen geäußert werden können, aber dennoch zu widersprechen und parteiisch zu sein, hat Nora Sternfeld anhand der Geschichtsvermittlung als notwendige Bedingung für eine agonistische Kontaktzone gefasst, eine Vermittlungssituation, in der Dissens und Multiperspektivität in einem gemeinsamen Raum verhandelt werden kann (Sternfeld 2012, 125). Die Paradoxie dieser Handlungsaufforderung trat bei Making Democracy deutlich zutage. Eine Möglichkeit, hier handlungsfähig zu bleiben, ist aus meiner Sicht, den Blick zu verschieben weg von Positionen hin zum Prozess, wie diese gebildet werden. Wäre es möglich, in einem Aushandlungsraum, wie wir ihn anstrebten, in den Vordergrund zu stellen, dass es für alle darum geht, sich eine Meinung zu bilden, und dazu Konfrontation ein nötiges Werkzeug ist? Das Üben und Ausüben der eigenen Meinung als Prozess war spannenderweise ein Punkt, der für einer/m Schüler*in an einem Projekt wie Making Democracy von Interesse war:

S1: »lch finde es eigentlich ganz gut, so ein making democracy, dass man immer darüber nachdenkt. Mal. Und wie man sich dann versucht, seine eigene Meinung einzubringen und versucht auch sich selbst zu bemühen, seine Kräfte so zu sammeln, dass man sie wirklich laut ausspricht." S2: »Finde ich eigentlich ganz klug.» (CS 24.4.2019)

Die Aufmerksamkeit liegt hier auf dem Ausdruck »Kräfte sammeln«: Es ist notwendig, Kräfte zu sammeln, um eine Meinung, über die man nachgedacht hat, auch auszusprechen. 


\section{Verschiebungen zum Aushandlungsraum}

Dass die Debatten über das Projekt und seine Rahmenbedingungen nicht abrissen, sich über das Schuljahr aber veränderten, liegt daran, dass sowohl dem Projektteam als auch den Schüler*innen klar war, dass Mitgestalten und Mitbestimmen in diesem Fall nicht ein Zusatzangebot oder Zugeständnis war, sondern dass partizipatorische Forschung von ihrer Mitgestaltung abhängig ist, und wir es also ernst meinen mussten. Wir wurden von den Schüler*innen beim Wort genommen und das Gegenspiel verlangte, unser eigenes Handeln immer wieder infrage zu stellen.

Demgegenüber reflektierten auch Schüler*innen ihr Handeln in eingespielten Rollen. Ein Schüler äußerte selbstkritisch gegen Ende des Schuljahres:

S2: »Also ihr fragt ja eh meistens, was könnten wir verbessern. Und dann ist es oft so, das bekommt ihr eh mit, dass einfach die ganze Klasse leise ist und nichts sagt.«

S1: »Und in Wirklichkeit regen wir uns halt dann auf.« (CS 24.4.2019)

Wenn auf diese Weise das eigene Handeln, der Schüler*innen und von uns, in den Strukturen zum Thema werden konnte, zeigte sich, dass die Verhandlung über das Projekt nicht eine Wiederholungsschleife war, sondern ein Prozess. Ein Aushandlungsraum kann nicht einfach proklamiert werden ihn zu erarbeiten war die zentrale Aufgabe in diesem Projekt. Und wenn die eingespielten und zugewiesenen Handlungsweisen auf einmal sichtbar wurden - als wir etwa über unsere Angebote im neoliberalen Zusammenhang reflektierten oder Schüler"innen realisierten, dass ihr Schweigen Zustimmung ist und nicht unbedingt Widerstand -, dann hat sich dieser Raum etabliert, und konnte temporär zu einem »Übungsraum« werden. Dieser blieb aber temporär und paradox, und bietet uns die Herausforderung als Vermittler*innen und Forschende, uns als Teil des Problems und weder als herausgehobene Beoachter*innen noch als per se ermächtigende Pädagog*innen zu reflektieren. 


\section{Literatur}

Bland, Derek/Atweh, Bill (2007). Students as researchers: engaging students' voices in PAR, in: Educational Action Research, Vol. 15(3), 337-349. https:// doi.org/10.1080/09650790701514259.

Bragg, Sara/Fielding, Michael (2005). It's an equal thing ... It's about achieving together: student voices and the possibility of a radical collegiality, in: Street, Hilary/Temperley, Julie (Hg.): Improving Schools Through Collaborative Enquiry, London/New York: Continuum, 105-135.

Crouch, Colin (2008). Postdemokratie, Frankfurt a. M.: Suhrkamp.

Duttweiler, Stefanie (2016). Nicht neu, aber bestmöglich. Alltägliche (Selbst) Optimierung in neoliberalen Gesellschaften, in: Aus Politik und Zeitgeschichte, (37-38), abrufbar unter: https://www.bpb.de/apuz/233468/ nicht-neu-aber-bestmoeglich-alltaegliche-selbstoptimierung-in-neoli beralen-gesellschaften?p=all (letzter Zugriff: 10.7.2019).

Gerdes, Jürgen/Bittlingmayer, Uwe H. (2016). Jugend und Politik. Soziologische Aspekte, in: Gürlevik, Aydin/Hurrelmann, Klaus/Palentien, Christian (Hg.): Jugend und Politik: Politische Bildung und Beteiligung von Jugendlichen, Wiesbaden: Springer VS, 45-67. https://doi.org/10.1007/97 8-3-658-09145-3_3.

Mouffe, Chantal (2008). Das demokratische Paradox, Wien: Turia + Kant.

Sauter, Sven (2006). Die Schule als Kampfplatz und als Aushandlungsraum. Über die soziale Bedeutung des Wissens aus der Perspektive der Cultural Studies, in: Mecheril, Paul/Witsch, Monika (Hg.): Cultural Studies und Pädagogik: Kritische Artikulationen, Bielefeld: transcript, 111-148.

Sternfeld, Nora (2012). Kontaktzonen der Geschichtsvermittlung. Wien: Dissertation.

Wöhrer, Veronika/Artzmann, Doris/Wintersteller, Teresa/Harrasser, Doris/ Schneider, Karin (2017). Spannungsfelder in der Forschungspraxis - eine Diskussion, in: dies.: Partizipative Aktionsforschung mit Kindern und Jugendlichen. Von Schulsprachen, Liebesorten und anderen Forschungsdingen, Wiesbaden: Springer VS, 11-26. https://doi.org/10.1007/978-3-65813781-6_2. 


\title{
Idee, Projekt, Vision und dann? Reflexion zum Thema »Demokratie machen«
}

Stefanie Schermann, Beate Wallner

\begin{abstract}
Making Democracy. Aushandlungen von Freiheit, Gleichheit und Solidarität unter Jugendlichen war ein Projekt, das Lernenden ihre eigenen Positionen in demokratischen Prozessen nahebringen und sie selbst als Gestalter*innen ihrer Gegenwart und Zukunft tätig werden lassen sollte. Konnte man dieser Vision gerecht werden? Und welches Klima entsteht, wenn Künstler*innen, Politikkundige, Heranwachsende und deren Erziehungsberechtigte durch ein gemeinsames Projekt Demokratie als ihr Recht begreifen und die Konsequenzen daraus leben? In diesem Beitrag möchten wir - zwei am Projekt beteiligte Lehrerinnen - auf die Projektidee eingehen und unsere Reflexion zur Umsetzung des Projektes am Schulstandort antonkriegergasse $e^{1}$ sichtbar machen.
\end{abstract}

\section{Rahmenbedingungen und pädagogische Motivation}

Auch wenn es obsolet erscheint, Rahmenbedingungen des schulischen Lernens hier darzulegen, da sie den meisten von uns selbstverständlich sind, sollen sie an den Anfang dieser Reflexion gestellt werden.

Ort, Zeit/Dauer/Stundenplan, Inhalt/Lehrstoffverteilung, Methoden, Überprüfung der Ergebnisse etc. sind durch Schulunterrichts- und Schulorganisationsgesetz, Haus- und Schulordnung, vertreten durch Lehrpersonal und Direktion, weitgehend festgelegt. Für kurze Phasen, Stunden oder Tage, ist ein Abweichen von dieser Struktur möglich. Dies muss jedoch von den Lehrenden beantragt werden. Diese strukturellen Vorgaben finden sich natür-

1 https://www.antonkriegergasse.at 
lich auch an der Schule antonkriegergasse. Die Schule, eine als Gymnasium gegründete Gesamtschule und Oberstufenrealgymnasium mit einer Vielfalt an Zweigen, ist bemüht, Lernenden unterschiedlicher Herkunft, Interessenlagen und Vorbildung Lebenschancen zu eröffnen. Sie hat sich in einem Letter of Interest ${ }^{2}$ dafür ausgesprochen, ihre Struktur für ein Projekt zu öffnen, das alltägliche Aushandlungsformen demokratischer Grundwerte untersucht.

In diesem Letter of Interest wurden vonseiten der Schule, in Vertretung einer Lehrperson, mehrere Fragen aufgeworfen, die sich mit dem Thema Politisierung und Demokratisierung in schulischen Zusammenhängen befassen und somit auf inhaltlicher Ebene die vorgegebene Struktur, sowohl schulisch als auch außerschulisch, untersuchen:

- Welche Vor- oder gar Nachteile entstehen für eine Gesellschaft, deren Mitglieder ihr Recht auf Teilhabe erkennen und einfordern?

- Wer profitiert von der heute konstatierten Politikmüdigkeit, dem Rückzug breiter Teile unserer Bevölkerung ins Private, Isolationistische?

- Was sagt es uns, dass, Umfragen zufolge, der Anteil der Personen, die einer »autoritären $\mathrm{Kraft}$ mehr vertrauen als demokratischen Prozessen, stark im Steigen begriffen ist?

- Und was können wir als Institution dagegen unternehmen?

- Welches Klima entsteht, wenn Künstler*innen, Politikkundige, Heranwachsende und deren Erziehungsberechtigte durch ein gemeinsames Projekt beziehungsweise durch gemeinsames Tun Demokratie als ihr Recht begreifen und die Konsequenzen daraus leben?

Darüber hinaus stellt sich die Frage, welche Interessen eine Schule dazu bewegen, an einem Projekt teilzunehmen, das Lernenden ihre eigenen Positionen in demokratischen Prozessen nahebringt und sie selbst als Gestalter"innen ihrer Gegenwart und Zukunft tätig werden lässt? So entwickelt sich ein Projekt, das viele Strukturen infrage stellt, obwohl die Schule selbst in einer tradierenden Struktur verhaftet ist.

Abgesehen von einem grundlegenden Anliegen der am Projekt teilhabenden Personen und dem Geist der antonkriegergasse lässt sich das Interesse

2 In einem Letter of Interest bekundet eine Schule Interesse an einem Sparkling-ScienceProjekt. 
auch mit Unterrichtsprinzipien und rechtlichen sowie pädagogischen Vorgaben begründen. $\$ 2$ des Schulorganisationsgesetzes lautet wie folgt:

»Die jungen Menschen sollen zu gesunden und gesundheitsbewussten, arbeitstüchtigen, pflichttreuen und verantwortungsbewussten Cliedern der Gesellschaft und Bürgern der demokratischen und bundesstaatlichen Republik Österreich herangebildet werden. Sie sollen zu selbständigem Urteil, sozialem Verständnis und sportlich aktiver Lebensweise geführt, dem politischen und weltanschaulichen Denken anderer aufgeschlossen sein sowie befähigt werden, am Wirtschafts- und Kulturleben Österreichs, Europas und der Welt Anteil zu nehmen und in Freiheits- und Friedensliebe an den gemeinsamen Aufgaben der Menschheit mitzuwirken.« (§2 SchOC)

Dieser Paragraph enthält die grundlegendsten Bestimmungen der Schule. Er impliziert eine Normierung, deren oberstes Ziel es neben der Bescheinigung einer vom Staat festgelegten Allgemeinbildung ist, freie und mündige Staatsbürger*innen, die das in sie investierte Kapital bestmöglich umsetzen, heranzubilden. Ein weiteres Resultat dieses Erziehungsprozesses sollte der/ die künstlerisch-kreative Gestalter"in seiner/ihrer gegenwärtigen und zukünftigen Welt sein, was durch die Formulierung »[...] befähigt werden, am Wirtschafts- und Kulturleben Österreichs, Europas und der Welt Anteil zu nehmen« ausgedrückt wird.

Nun liefert ein Blick auf die zu Beginn angeführten rigiden Begrenzungen, in denen eine solche Entwicklung vorgeblich stattfinden soll, die Erkenntnis, dass dieser Rahmen ein zu enger sein mag. So betrachtet scheint es fast ein Ding der Unmöglichkeit zu sein, beide durchaus konträre Ziele - das des ökonomisch ausgerichteten Produzenten und Konsumenten einerseits und das des freien und kreativen Gestalters seiner Lebenswelt andererseits durch eine Einrichtung wie die öffentliche Schulpflicht beziehungsweise das öffentliche Schulwesen zu erreichen. Geht man davon aus, dass ein Widerspruch besteht zwischen dem/der angepassten Staatsbürger*in, der/die sich ökonomisch kalkulierbar verhält, und jenem freien, selbstbestimmten kreativen Wesen, wird deutlich, für welches Menschenbild sich der Staat entschieden hat: jenes der pflichtbewussten Steuerzahler*innen. Trotz aller Aufforderung zum kritischen Denken und kritischen Konsumieren steht wirtschaftliches Wachstum unangefochten im Zentrum des globalisierten, westlichen Lebensstils. 
Jugendliche sind jedoch für kritische Ideen diesem Normierungsprozess gegenüber zweifellos zu gewinnen, wie die Entwicklung der Klimaschutzbewegung (Fridays for Future) seit Herbst 2018 deutlich vor Augen führt. Einschnitte im eigenen Konsumverhalten vorzunehmen fällt den meisten von ihnen aber ebenso schwer wie uns Erwachsenen. Und die Flucht vor deutlich wahrnehmbaren Problemen unserer Gesellschaft in virtuelle Welten ist so verbreitet und lebensbestimmend wie nie zuvor.

Diesen Bestimmungen, Begrenzungen, Normierungen einerseits verpflichtet, andererseits dem eigenen pädagogischen Denken und Handeln verbunden, scheint es zum Teil widersprüchlich, dass sich Lehrpersonen, die kurze Zeit oder viele Jahre im Schulsystem zugebracht haben, über tatsächliche Demokratisierungstendenzen im aktuellen schulischen Kontext kritisch äußern. Wir aber halten an der Idee der staatlichen Schule fest, nicht wegen ihres starren Rahmens, sondern dennoch. Was Schule für uns ausmacht, ist das Wie, nämlich wie in diesen Strukturen gelebt wird beziehungsweise wie trotz aller Normierung ein freies, kreatives Miteinander möglich ist. Dieses Miteinander des Sehens, Wahrnehmens, Wertschätzens, Zutrauens, Vertrauens und lebendigen Seins - das ist für uns Schule, tägliches Lernen und Scheitern, Aufstehen und Weitermachen.

Die Basis für diesen Zugang ist viel gemeinsam verbrachte Zeit, Großzügigkeit, sich und anderen gegenüber, eigenem und fremdem Fehlverhalten gegenüber, und die Fähigkeit, an diesen Gegebenheiten weiterzuarbeiten. Und dabei Dogmatismus hintanzustellen.

\section{"Demokratie machen« - Projektidee und Projektverlauf}

Was haben diese abstrakten Überlegungen mit unserem Making DemocracyProjekt zu tun? Viel, finden wir! Denn ohne die beschriebene Vorstellung über Demokratie und die Einstellung zum schulischen Miteinander wäre eine Projektdurchführung nicht anzudenken gewesen.

Die Projektbeschreibung äußerte die Idee, alltägliche Aushandlungsformen zentraler, demokratischer Grundwerte, nämlich Freiheit, Gleichheit und Solidarität, durch/mit Jugendliche/n zu untersuchen. Wichtig im Hinblick auf diese Idee ist der Grundgedanke, dass diese Werte nicht allein im politischen System, sondern auch im Alltag der Menschen laufend neu verhandelt werden und auf diese Weise erst Demokratie gemacht wird (Ebner von Eschenbach 2016). 
Schon die Bezeichnung Demokratie machen sagt, genauso wie politische Bildung, dass man etwas über Demokratie und Politik lernen kann. Dies stellt die Theorie der demokratischen und politischen Bildung vor nicht geringe Schwierigkeiten, wenn sie sich von vorgegebenen Strukturen, auch im schulischen Rahmen, abwendet und einer Perspektive der Freiheit zuwendet. Es gehört zu den Intentionen und Leitgedanken der politischen Bildung - die überdies als Unterrichtsprinzip im Schulwesen existiert - mit ihren Angeboten Schüler*innen dabei zu unterstützen, sich Gedanken über Politik zu machen. Demokratiebildung kann diese Unterstützung im Grunde genommen nur durch das Angebot von Gelegenheiten zu einer ernsthaften und von professionellem Personal begleiteten Auseinandersetzung leisten (Sandner 2008, 58). trafo.K, diverCITYLAB, Ka Schmitz, das Projektteam der Universität Wien und weitere Akteur"innen machten solche Angebote: $\mathrm{zu}$ forschen, infrage $\mathrm{zu}$ stellen, neue Positionen einzunehmen, auszuprobieren. Die Schüler*innen nahmen sie in den beiden Projektjahren zu einem großen Teil bereitwillig wahr. Einige Arbeits- oder Gedankenprozesse, die aus den Angeboten resultieren, konnten sichtbar gemacht werden. Die Umsetzung der gewählten Themen trug eindeutig die Handschrift der Schüler"innen, dies war im Mai 2018 ersichtlich bei der ersten Präsentation im Dschungel Wien - Theaterhaus für junges Publikum. Ein Teil dieser Präsentation aus dem ersten Projektjahr soll hier einfließen, nämlich ein Ausschnitt aus dem Song Hut des Lebens.

Hut des Lebens

Wo ist das Licht?

Man sieht es nicht.

Kann ich's einschalten?

Nein, du musst dich zurückhalten. [...]

Es gibt so viele Dinge, die mich ärgern.

Besserwisser und Ceschwister und natürlich Eckenpisser, [...]

Sockenfressende Waschmaschinen. [...]

Wenn alle in ihr Handy glotzen,

dann ist die ganze Welt zum Kotzen.

Das sind die Dinge, die uns ärgern,

und unser Leben sehr erschweren.

Das sind die Dinge, die uns ärgern,

drum wollen wir uns heute recht empören.

Minecraft ist viel einfacher als das echte Leben, 
man könnte davon schon was abschauen. [...]

Ich leb' doch lieber im echten Leben,

da gibt's viel coolere Sachen. [...]

Aber eigentlich geht's uns gut.

Wir bringen alles unter einen Hut. [...]

(Pascal Wojner und Emil Smekal, Schüler aus dem ersten Projektjahr)

Diese Textpassage zeigt, dass den Schüler*innen ein Raum zur Wissensaneignung eröffnet wurde, der ihrer Lebenswelt entspricht. Sie konnten sich mit Alltagsdemokratie auseinandersetzen und ihre Gedanken affektiv sowie performativ verarbeiten. Klar war: Dieses Projekt wurde für sie entwickelt, die Gestaltung lag in ihren Händen. Ihre Neugier und Freude sich zu zeigen, waren bestimmende Faktoren für das Gelingen des Projektes.

\section{Zusammenarbeit als Aushandlungsform demokratischer Grundwerte}

Der im eingangs erwähnten Letter of Interest ausgedrückte Wunsch, Demokratisierung müsse bedeuten, Schüler*innen ihre tatsächlichen Bedürfnisse erkennen zu lassen und sie zu entsprechenden Taten zu ermutigen, sie selbst als Gestalter*innen ihrer Gegenwart und Zukunft tätig werden zu lassen, birgt im staatsschulischen Rahmen einiges an Sprengkraft, die sich nicht nur in Handlungen, sondern ebenso sublimer Verweigerung Bahn bricht. Und warum auch nicht? Es ist ja ein von Erwachsenen erdachtes Projekt und den Handlungsspielräumen der Schüler*innen, wie wohl zahlreicher als im Regelunterricht, stehen klare Grenzen entgegen. Genau aus diesem Grund sollte die Zusammenarbeit aller Beteiligten als Aushandlungsform demokratischer Grundwerte gesehen werden.

Diese Zusammenarbeit war im Vorfeld des Prozesses fixiert, unter anderem durch die Projektbeschreibung. Der Anspruch war, dass Wissenschaftler*innen, Vermittler*innen, Künstler*innen, Lehrer*innen und Schüler*innen als Projektteam zusammenarbeiten und der methodische Zugang durch Alltagsnähe, Multiperspektivität und Prozessorientierung gekennzeichnet ist. Die Machtverhältnisse im Team sollten für alle Beteiligten thematisierbar und nicht verdeckt sein. Zusätzlich sollte die Projektarbeit in zwei Durchgängen realisiert werden, wobei mindestens acht Workshoptermine vorgesehen waren, 
in denen es von der Erprobung unterschiedlicher Forschungsmethoden zu einer Vergegenständlichung und Sichtbarmachung der Ergebnisse kommen sollte. Letztlich wurden in jedem Schuljahr 15 Workshops durchgeführt.

In der Reflexion stellt sich nun heraus, dass vor allem im zweiten Projektjahr nicht die vermittelten oder erarbeiteten Inhalte die hauptsächliche Aufmerksamkeit der Schüler*innen erhielten, sondern die Zusammenarbeit an sich als zentrales Anliegen galt. Die am zweiten Durchgang teilnehmenden Schüler*innen im Alter zwischen 16 und 19 Jahren unterlagen trotz ihres allgemeinen politischen Grundinteresses, das sich nicht nur im schulischen Alltag zeigt und mit unterschiedlichen Publikationen bestätigt wird (Großegger/Rohrer 2015), und trotz der mit dem Projekt einhergehenden Öffnung der Unterrichtsstruktur Normierungen im Schulalltag. Damit sind Strukturen gemeint, die in der Zeit des Regelunterrichts massive Präsenz im Alltagsleben der Lernenden einnehmen: Schularbeiten, Tests und der damit einhergehende Leistungsdruck. Und nicht nur das: Im Grunde genommen haben sie auch durch die Projektteilnahme Zwang erfahren. Sie wurden von der Lehrkraft dazu angehalten, an diesem Projekt als Klasse teilzunehmen. In diesem Kollektiv wurde das Machtverhältnis innerhalb des Projektteams oftmals thematisiert. Sogar von Streik und allgemeiner Verweigerung vonseiten der Schüler*innen war zeitweilig die Rede. Widerstand war spürbar und sichtbar - freiwillige Partizipation nur gering wahrnehmbar.

Dieser Widerstand oder das Überschreiten des vorgegebenen Möglichkeitsraumes innerhalb des Projektes kann und muss jedoch als positives Ergebnis des Aushandlungsprozesses von Demokratie im Alltag gesehen werden, da die Ordnung auf die Probe gestellt wurde und alternative Gestaltungsperspektiven sichtbar gemacht wurden. Diese Art gesellschaftlicher Widerständigkeit ist historisch hinreichend dokumentiert und "gilt als unverzichtbares Kernstück für Demokratisierung (Ebner von Eschenbach 2016, 3). Zusätzlich wurde allen Projektbeteiligten deutlich, was es heißt, politische (Un-)Freiheit im Alltag zu leben.

»Es erfordert von den Menschen auch Anstrengung und Unbequemlichkeiten: das Anderssein der anderen auszuhalten etwa, im Dissens und in einer Minderheitenposition leben zu können, Kompromisse schließen zu können, Geduld und Ausdauer zu entwickeln, um andere von der eigenen Position zu überzeugen, die Souveränität, sich unter Umständen von anderen überzeugen zu lassen.« (Sander 2008, 54) 


\section{Reflexion der sozialen Beziehungen unter den Forschungspartner*innen}

Welches Klima entsteht daher, wenn Heranwachsende durch ein gemeinsames Projekt Demokratie als ihr Recht begreifen und die Konsequenzen daraus leben? Der Widerspruch initiierte vor allem im zweiten Durchgang eine Reflexion der sozialen Beziehungen unter den Forschungspartner*innen, wobei den Lehrkräften eine besondere Rolle zukommt, da die Schüler*innenLehrer*innen-Beziehung eine andere ist als jene zu den anderen Projektbeteiligten. Trotz des Widerstandes der Schüler*innen kam es zu keinem Abbruch des Projektes. Dies mag zuerst verwundern, kann aber mit genau dieser Schüler*innen-Lehrer*innen-Beziehung in Verbindung gebracht werden. Denn Sinn erhalten Schüler*innen und ihr Tun sowie ihr Lernen »nur von konkreten Personen, mit denen sie konkrete Erfahrungen machen können, von Menschen, die sich ihnen zuwenden und die - weil sie an sie glauben von ihnen auch etwas fordern«, und Erziehung und Bildung bauen auf mit realen Personen gemachten Erfahrungen auf (Bauer 2008, 142). Das Fordern und Entgegenkommen der Lehrkraft war deshalb mitunter ausschlaggebend für die Beilegung des Widerstandes und der wieder aufgenommenen Sinnzuschreibung des Projektes.

Dies mindert jedoch keineswegs die Rolle aller Forschungspartner*innen. Innerhalb des Projektes gab es Diskussionen, Reflexionen, Erfahrungen, die auf sozialer Ebene den Prozess des »Demokratiemachens « maßgeblich beeinflusst haben. Auffallend war, dass den Schüler*innen persönliche soziale Anerkennung und Wertschätzung im sozialen Miteinander besonders wichtig waren und sie diese, wenn notwendig, auch zur Diskussion gestellt beziehungsweise eingefordert haben. Die Anerkennung und Wertschätzung bezogen sich allerdings nicht nur auf ihre Person und Stellung innerhalb des Projektes, sondern auch auf ihre (politische) Meinung. Ausgehend davon muss festgestellt werden, dass ein aktiver Prozess der Ausverhandlung der eigenen Position der Schüler*innen innerhalb des Projektes stattgefunden hat und sie dadurch vorherrschende Macht- und Normierungsverhältnisse kritisiert sowie hinterfragt haben. 


\section{Projektergebnisse und Vision}

Im schulischen, aber auch im außerschulischen Bereich erwartet man nach einem Projekt auch Projektergebnisse, die sichtbar für Beteiligte und Außenstehende sind. Demokratie und das Demokratiemachen können jedoch bloß punktuell sichtbar gemacht werden. Die Theaterperformance und die Ausstellung von Fotos und Graphic Novels im Rahmen der Fachtagung zeigen nur einen kleinen Ausschnitt. Soziale Prozesse sowie viele individuelle Gedanken entziehen sich der Darstellung und bleiben unsichtbar. Auch die vorangegangenen Ausführungen können nur einen kleinen Teil des Prozesses und den für die Schüler*innen gewonnen Mehrwert aus Sicht der Lehrer*innen zeigen.

Am besten lässt sich dies anhand einiger Statements von Schüler*innen veranschaulichen, in diesem Fall aus dem ersten Projektdurchgang. Diese Aussagen der Schüler*innen sollen hier nicht mehr diskutiert und erläutert werden, da unseres Erachtens eine Unzulänglichkeit durch die Erklärung aus einer anderen Perspektive, im konkreten Fall jener der Lehrerinnen, gegeben wäre.

»Ich hatte bei dem Theaterstück ja eine ziemlich verrückte Rolle und dachte, dass sich viele darüber lustig machen würden, aber ich habe sehr viel Lob und Zuspruch bekommen. Das hat mich sehr gefreut, denn wenn man Demokratie gut darstellt, werden Leute auch darauf aufmerksam. Es hat generell sehr viel Spaß gemacht, ein Teil dieses Projektes zu sein und viel darüber zu lernen! Ich habe auf jeden Fall viel gelernt und habe viel mitgenommen.« "Das Making Democracy-Projekt hat mir gut gefallen, da die Betreuerinnen und Betreuer sehr nett waren, die Ergebnisse der beiden Klassen sehr gut waren und ich der Message, die dahintersteht, zustimme. Was ich auch gut fand, war, dass unsere Betreuerinnen und Betreuer uns viel beigebracht haben, wie zum Beispiel, dass es verschiedene Wertvorstellungen gibt. Momente, die mir besonders in Erinnerung geblieben sind, waren der Theaterabend im Museumsquartier, der Besuch im Parlament und die Interviews in der Universität Wien.«

Der Gewinn lag in diesem Projekt auf der Betonung der künstlerisch-kreativen, freien und mündigen Staatsbürger*innen. Diese Facette kommt in der Schule, aufgrund von Normierung, Anpassung und Druck, oft zu kurz. So 
kann der Mehrwert folgendermaßen formuliert werden: Freiheit wurde gespürt. Das ist doch einiges. Und mehr davon ist unseren Schüler*innen und Lehrer*innen auch in Zukunft zu wünschen.

\section{Literatur}

Bauer, Joachim (2008). Lob der Schule, München: Heyne.

Ebner von Eschenbach, Malte (2016). Doing Difference - Die Reflexion auf Unterscheidungen als Ansatz Politischer Erwachsenenbildung, in: Magazin erwachsenenbildung.at. Das Fachmedium für Forschung, Praxis und Diskurs, Ausgabe 28, abrufbar unter: https://www.erwachsenenbil dung.at/magazin/16-28/meb16-28.pdf (letzter Zugriff: 12.5.2019).

Großegger, Beate/Rohrer, Matthias (2015). Jugend und Politik - Repräsentativumfrage unter 14- bis 18-jährigen ÖsterreicherInnen, Eigenstudie des Instituts für Jugendkulturforschung.

Sandner, Günther (2008). Politik entdecken - Freiheit leben. Didaktische Grundlagen politischer Bildung, Schwalbach: Wochenschau.

SchOG - Schulorganisationsgesetz (1962), abrufbar unter: https://www.ris. bka.gv.at/GeltendeFassung.wxe?Abfrage=Bundesnormen\&Gesetzesnum mer=10009265 (letzter Zugriff: 15.5.2019). 


\title{
Wie utopisch ist es, von einem demokratischen Theater und einem demokratischen Lernen zu reden? Übungen in zwei Systemen
}

Aslı Kışlal

\begin{abstract}
In den Programmen der Theaterbühnen wird der Eindruck vermittelt, dass die Demokratie gepriesen, neu erfunden und Systeme infrage gestellt werden. Blickt man hinter die Kulissen, wird klar, wie undemokratisch es am Theater als Ort und Betrieb zugeht. Ist Theater demokratisierbar? Und ist Lernen demokratisierbar? diverCITYLAB ist ein künstlerisch-politisches Projekt, getarnt als Schauspiel- und Performance-Akademie, und war Partner im Projekt Making Democracy. Der Beitrag diskutiert anhand der Arbeit von diverCITYLAB die Versuche, im System Theater und im System Schule kollektive Übungsräume für Freiheit zu schaffen.
\end{abstract}

Das diverCITYLAB war zwei Jahre lang Partnerinstitution von Making Democracy. Der Beitrag bestand darin, mit den Schüler*innen über die Formen der Präsentation zu diskutieren und diese zu erarbeiten. Zuvor hatten die Schüler*innen inhaltliche Diskussionen geführt und sich monatelang mit dem Thema Making Democracy auseinandergesetzt. Dann kamen wir ins Spiel und versuchten das, was sie bewegte, ihre Gedanken und Wünsche, ihre Ideen, in eine performative Form $\mathrm{zu}$ bringen.

Im ersten Jahr (2017/18) haben wir gemeinsam mit circa 50 Schüler*innen im Alter von zwölf bis dreizehn Jahren eine theatrale Auseinandersetzung über den Begriff Demokratie entwickelt. Wir behandelten Fragen wie: Was versteht ihr unter Demokratie? Welche Strukturen eurer Umgebung (Schule, Familie) bezeichnet ihr als demokratisch? Im zweiten Jahr (2018/19) arbeiteten wir mit siebzehn Schülerinnen im Alter von siebzehn Jahren und aufwärts. Bei diesem Prozess begleiteten uns zehn Studierende des diverCITYLAB. 


\section{Theater als Raum der Freiheit?}

Bei dieser künstlerischen Arbeit prallten angeblich gegensätzliche Disziplinen aufeinander. Sascha Willenbacher hat die Zusammenarbeit zwischen Theater und Schule in seinem Bericht zum Projekt JUMP \& RUN analysiert (Willenbacher 2016). Er erwähnt, dass schon bei der Formulierung des Projektkonzepts durch die Kunstinstitutionen eine hierarchisierende Abgrenzung zwischen Kunst und Schule stattfindet. Beide Seiten (Lehrer*innen und Künstler*innen) sehen einander als das Andere und positionieren sich gegenüber ihrem Kooperationspartner mit gewissen Vorannahmen. So beschreiben die Konzeptschreiber*innen der großen Theaterinstitutionen ihre Kunst und sich selbst in einer vermeintlichen Vorreiterrolle, während der Schule und den Lehrer*innen eine defizitäre Rolle zugeschrieben wird:

»Im Konzeptpapier, so lässt sich daraus ableiten, gehen die beteiligten Theater implizit davon aus, dass die von ihnen mit Kunst verknüpften Wirkungsabsichten (>gesellschaftliche Irritation und Ermächtigung zur Artikulation eigener Positionen`) im Kontext Schule bislang nicht oder zumindest nicht ausreichend zum Zuge kommen. Die Theater treten via Konzeptpapier mit der Forderung nach selbstreflexiver Auseinandersetzung an die Schulen und damit an die Schüler_innen heran.«(Willenbacher 2016, 203)

Das äußere sich in Konzepttexten wie dem Folgenden, den Willenbacher zitiert:

»Eine weitere Besonderheit ist die gemeinsame thematische Grundlage, auf der die Projektideen der Lehrer/Künstler-Teams entstehen. Es geht um die Auseinandersetzung mit der Institution Schule, mit ihren offiziellen und inoffiziellen Regelwerken, mit ihrer Funktion für die Gesellschaft, mit ihrer Aufgabe zur Disziplinierung des Einzelnen, der Vermittlung von Fähigkeiten [...] [und] um die Frage, wie die Institution Schule auf das Individuum wirkt und umgekehrt. [...] Kunst als Aneignung von Lebenswirklichkeiten und gesellschaftlicher Diskurse. Dies gilt es, in actu den Lehrern und Jugendlichen zu vermitteln.«(Harmsen et al. 2010, 44, zit. n. Willenbacher 2016, 203 f.)

Das Theater, die Kunst und die Künstler*innen als Lebensretter*innen? Wenn man sich die Jahresprogramme der Theaterbühnen ansieht, gewinnt 
man den Eindruck, es werde dort die Demokratie gepriesen, Neues erfunden und Alltagspraxen infrage gestellt. Wenn man hinter die Kulissen sieht, wird klar, dass das Theater de facto ein undemokratischer Ort und Betrieb ist.

Wie steht es zum Beispiel um Frauen in höheren Positionen? Ab der Saison 2021/22 werden von den zwölf großen Sprechtheaterbühnen in Österreich nur mehr drei von einer Intendantin geleitet werden. ${ }^{1}$ Davon ist keine einzige aus der (post-)migrantischen Künstler*innen-Community. Zwei Frauen, die aktuell Leitungspositionen innehaben, werden demnächst von Männern ersetzt.

In der Saison 2018/2019 wurde im Theater in der Josefstadt von 15 Stücken, die Premiere hatten, kein einziges von einer Autorin geschrieben. Im Burgtheater wurden in der gleichen Saison von 21 Inszenierungen nur fünf von Regisseurinnen inszeniert, drei davon auf der kleinsten Spielstätte (Vestibül). Von insgesamt 157 Produktionen im Jahr 2018/2019 wurden nur 57 von Frauen inszeniert (das entspricht 37 Prozent aller Produktionen in den zwölf großen Häusern), davon nur zwei von Regisseurinnen aus der (post-)migrantischen Künstler*innen-Community.

In den Schauspielschulen werden meist Klassiker mit ihren verstaubten Geschlechterrollen gelehrt. Nach wie vor greifen Theater zum Blackfacing und argumentieren das mit künstlerischer Freiheit. Die Türken spielen im Theater nur Türken, aber ein Österreicher kann einen Engländer, einen Spanier, einen Mauretanier oder sogar einen Tisch spielen - und wird nie infrage gestellt.

Warum also benutzen Theatermacher*innen Demokratie lediglich als Stoff ihrer Inszenierungen, setzen sie aber nicht um? Ist Theater demokratisierbar? Wie können wir Kunst als verbindendes Element sehen? Gegen Vereinsamung, gegen rechte Parolen, zum Denken anregend. Theater oder Kunstinstitutionen wären als Begegnungszonen zu etablieren - nicht elitär, aber auf künstlerischer Ebene die Sehgewohnheiten kritisch überdenkend. Von uns Theatermacher*innen wurde vernachlässigt, das Publikum zu einer Entwicklungsreise mitzunehmen. Wer sich nicht dauerhaft im Theaterkontext

1 Dank an Bérénice Hebenstreit und Michael Isenberg, die ihre privaten Recherchen über den Anteil der Frauen in entscheidenden Positionen (Leitungsposten, Regieposten und Autorschaft an den zwölf größten Theatern Österreichs) zur Verfügung gestellt haben. Weiterführende Informationen unter https://mosaik-blog.at/theater-oesterreich-ungleich-ge schlecht-frauen-maenner/ (letzter Zugriff: 25.7.2019) und https://mosaik-blog.at/nestroytheater-sexismus-geschlechter/ (letzter Zugriff: 25.7.2019). 
bewegt, fühlt sich von den Angeboten entfremdet; belehrt, doch nicht berührt. Wie schaffen wir Berührung im physikalischen und psychologischen Sinne?

Kunst ist ein Grundbedürfnis. Ich würde sogar die Allgemeine Erklärung der Menschenrechte mit einem »Recht auf Kunst« erweitern wollen. Wenn man die (post-)migrantische Realität als gegeben anerkennt, kann das Theater, das diese Realität reflektiert, daran wachsen. Das beinhaltet sowohl neue Themengebiete als auch neue Gesichter, die diese Gesellschaft seit langem bietet, also neue Regisseur*innen, Schauspieler*innen, Dramaturg*innen, Autor*innen.

Es braucht eine Dekolonisierung, um die Kunst von ihrem kolonialistischen Denken zu befreien, das wie oben geschildert oft sehr ausgeprägt ist. Herkunft, Sprache und äußeres Erscheinungsbild sind bedeutende Faktoren im Theater. Durch Sehgewohnheiten entstehen Normierungen, die wir nicht mehr hinterfragen, auch wenn sie mit der Realität, die uns umgibt, wenig zu tun haben. Wir sollten uns aber daran gewöhnen, dass wir gemeinsam im 21. Jahrhundert leben. Dem Theater sollten wir zugestehen, eine Welt zu erdenken, in der wir leben wollen. Gerade dann, wenn diese Welt außerhalb des Theaters nicht die Welt ist, in der wir leben wollen.

Und wenn Tausende Menschen jeden Donnerstag über Monate auf den österreichischen Straßen demonstrieren ${ }^{2}$ und dies wie eine lebendige Performance wirkt, wo jede"r eine Stimme hat, wie kann ein Theater als Sprachrohr eine Bewegung etablieren? Die Arbeit von diverCITYLAB setzt an dieser Frage an.

\section{Strategien der Demokratisierung: ein künstlerisch-politisches Projekt, getarnt als Performance-Akademie}

diverCITYLAB ist ein künstlerisch-politisches Projekt, getarnt als Schauspiel- und Performance-Akademie. Es braucht neue Strategien, um Identitäten zu schaffen, die sich aus der Diversität ihrer Individuen stärken, anstatt diese zu fürchten. Der Begriff postmigrantisch versucht die Realität aller Individuen, die diese Gesellschaft bilden, zu beschreiben und die Trennlinie zwischen dem »Wir« und dem »Ihr« aufzuheben.

2 Vgl. https://wiederdonnerstag.at/medienberichte/ (letzter Zugriff: 13.7.2019). 
diverCITYLAB versteht acting als reacting. Wir wollen dem Gegenwartstheater ein neues, unserer postmigrantischen Gesellschaft angemessenes Gesicht geben, mit neuen Akteur*innen und einem Publikum, das sämtliche Beteiligten unserer Gesellschaft einschließt. Um es dem Kunstbetrieb langfristig zu ermöglichen, mit dem gesellschaftlichen Wandel mitzuhalten und somit eine nachhaltige Veränderung in der Szene anzustoßen, ist es wichtig, denjenigen, die in der Kunst ihre Zukunft sehen, diese Gelegenheit zu bieten.

$\mathrm{Zu}$ diesem Zweck entstand Ende 2013 das diverCITYLAB - THEATER-, FILM- und PERFORMANCElabor. Das Ziel dieser Synthese aus Kunstprojekt und praxisorientierter Ausbildungsstätte ist, die darstellenden Künste für alle Mitglieder unserer postmigrantischen Gesellschaft zu öffnen.

Unsere Tätigkeit umfasst drei Bereiche. Der umfangreichste Bereich ist die diverCITYLAB Schauspiel- und Performance-Akademie, ein vierjähriges künstlerisches Ausbildungsprogramm. Alle zwei Jahre startet ein neuer Jahrgang die kostenlose Ausbildung. Die Finanzierung des Projekts erfolgt hauptsächlich über die Förderung der Kulturabteilung der Stadt Wien.

Wir haben uns gegen eine klassische Aufnahmeprüfung zur Ausbildung entschlossen, da dies den Zugang erschweren würde (etwa wegen Sprachbarrieren oder fehlenden finanziellen Möglichkeiten für Privatunterricht). Die Auswahl der Studierenden findet nach einer offenen Workshop-Phase statt. Nach dieser dreimonatigen Phase zeigen die Beteiligten in einer Performance, was sie erlernt haben. Danach suchen wir die zukünftigen Studierenden aus. Dabei ist uns wichtig, dass die Beteiligten für diesen möglichen Beruf »brennen«. Die Ausbildung umfasst fünfzehn bis achtzehn Stunden Unterricht pro Woche. Sie beinhaltet praktische und theoretische Wissensvermittlung. Vor allem wird versucht, ein kritisches künstlerischpolitisches Denken in den Unterricht einzuarbeiten. Das bedeutet auch eine Auseinandersetzung mit dem herkömmlichen Theaterverständnis. Schauspieltheorien werden gelehrt und gleichzeitig hinterfragt. Abgesehen von der durch die Dozent"innen qualitativ hochwertigen Schauspielausbildung sind gesellschaftliche Verantwortung sowie die Positionierung in unserer Gesellschaft zentrale Themen der diverCITYLAB-Akademie. Wir bilden eigenständig denkende und handelnde Schauspieler*innen aus, die jeweils sensibel für neue Theaterformen und gesellschaftliche Entwicklungen sind. Wir verstehen Schauspieler*innen als autonome Künstler"innen und vermitteln ihnen über die Schauspieltechniken hinaus Fähigkeiten, die in der 
zunehmend genreübergreifenden, performativen, kollektiven Theaterarbeit vonnöten sind.

Der zweite Bereich von diverCITYLAB ist das Artist-in-Residence-Programm. Um internationalen Kunstschaffenden, die Österreich als ihre Wahlheimat sehen, einen Einstieg in die Kunst- und Theaterszene zu ermöglichen, unterstützen wir diese bei ihren Projekten. Diesen neu nach Österreich gekommenen Menschen fehlt jegliche Lobby und Vernetzung im hiesigen Kunstbetrieb. Sie nehmen Ausschreibungen nicht wahr und kennen die Förderinstrumente nicht. Daher ist unsere Vermittlung zu den unterschiedlichen Communities und NGOs von großer Bedeutung. Derzeit arbeiten wir daran, auch geflüchtete Künstler*innen in das Projekt aufzunehmen, um ihnen ein Forum und Vernetzungsmöglichkeiten zu bieten. Zwei Künstler"innen jährlich können wir intensiv betreuen und sie zu unseren Stipendiat*innen machen. Dieses Programm reicht über ein klassisches Stipendienprogramm hinaus, da allein die sprachlichen Hürden das Arbeiten erschweren. Neben der finanziellen Unterstützung der Stipendiat*innen bieten wir Hilfe beim Übersetzen von Texten, dramaturgische Betreuung sowie Beratung bei produktionstechnischen Fragen an und helfen mit der Bereitstellung von Räumen und der Vernetzung mit anderen Institutionen und Initiativen.

Ein dritter Bereich von diverCITYLAB sind die Kunstvermittlungsprojekte, die wir gemeinsam mit Sozialpartnern, Frauenorganisationen und Schulen erarbeiten. Wir versuchen durch unsere Arbeit, die Angst vor Kunst zu nehmen und Diversität positiv zu besetzen. Wenn Menschen sich erst einmal mit Kunst beschäftigen, ist die Chance groß, dass sie ein weiteres Interesse entwickeln. Nicht jede"r muss Künstler*in werden, aber jede*r hat das Recht, Kunst zu genießen. Ohne Zugang zur Kunst bleibt diese ein abstraktes Gebilde, das kaum jemanden anspricht. Daher ist unser Anliegen, mit praxisorientierten Projekten ein neues Publikum fürs Theater $\mathrm{zu}$ begeistern, den sehr unbeweglichen Raum Theater zu den Menschen zu bringen, anstatt $\mathrm{zu}$ warten, dass sie sich ins Theater begeben. Um Theater entstehen zu lassen, braucht es die großen Institutionen nicht, sondern nur die Gasse, in der man lebt, den Schulhof, oder einen Park, wo man gerne sitzt. 


\section{Zwischen den Systemen}

Making Democracy war für unsere Akademie-Studierenden genau das richtige Projekt. Schließlich erhalten sie vier Jahre lang eine Ausbildung in einem Handwerk und dessen Techniken, also ist es wünschenswert, diese Erfahrungen in soziales Engagement umzusetzen, um das Gelernte der nächsten Generation weiterzugeben. Zudem verbindet ein solches Projekt die Klassengemeinschaft der Studierenden, hält sie offen, tolerant, aufmerksam und wach, sodass sie sich mehr mit ihrem Umfeld beschäftigen, was sie wiederum in ihrem künstlerischen Tun bestärkt.

Dabei haben wir eines unterschätzt: Viele unserer Studierenden sind selbst erst vor wenigen Jahren aus dem System Schule entlassen worden. Die Begegnung mit der Schule kann alte Wunden öffnen. Die Projektarbeit mit den Schüler*innen und den Studierenden hat daher Diskussionen im diverCITYLAB entfacht. Ein Ausschnitt aus der Kommunikation mit einer Studierenden kann die Situation nachvollziehbar machen:

»Ich kann mich nur wiederholen. Eine reine Zwangsbeglückung. Der ultimative Versuch, Jugendlichen in einem vergifteten System, an dem Schule nur eine weitere Auswucherung ist, etwas mühselig zu erarbeiten. Schule macht krank, Schule ist eine totale Vorbereitung für die kranke Welt da draußen. Schule ist eine Erziehungsanstalt. In der Schule wird gehorsam gelehrt, Individuen zum kollektiven gehorsamen erzogen, das denken wird ausgetrieben.

Und ein Schüler hat es sehr treffend formuliert: ein `freiwilliges` Projekt, was gar nicht aus Freiheit geboren ist.

Entschuldigung Asli, ich hasse dermaßen Schule. Ich kann mich nur zu gut erinnern, dass ich an Leistungen und Noten gemessen worden bin.

Und nochmal in einer Schule aufzutauchen, in der Position, Heranwachsende in eine Richtung zu führen, in einer Umgebung, wo ich mich nie frei entfalten konnte, ist für mich ein Widerspruch. Obwohl ich am Anfang mich gefreut hab, und vielleicht meine Versöhnung mit der Schule, durch eine andere Position in diesem System wieder einzutauchen gewünscht hab. Ich habe mich aber wieder in dieser Stresssituation gefunden, der mich schon damals wie ein Alptraum vorkam ...« (aus der Korrespondenz zwischen einer diverCITYLABStudierenden und Aslı Kışlal, Mai 2019) 
Wir sind an unsere Grenzen gekommen. Diese Grenzen haben aber auch konstruktive Diskussionen (eher spät, aber doch rechtzeitig) mit den Schüler*innen ermöglicht, in denen das Projekt generell und auch unsere Beteiligung infrage gestellt wurde.

In weiteren Gesprächen mit den Studierenden gaben sie selbst zu, dass ihr eigenes Verhalten und ihre Vorannahmen die Produktivität der Arbeit erschwert haben. Die Selbstverortung im Kunstbereich führte zu einem $\mathrm{Zu}$ gang im Sinne von »Jetzt gehen wir in die Schule und zeigen, wie die freie Welt funktioniert«. Dieser Zugang erwies sich von vornherein als Hindernis und wirkte herablassend.

Sascha Willenbachers Analyse über die Zusammenarbeit zwischen Schule und Kunstinstitutionen war für uns sehr zutreffend: Künstler*innen gehen in ein anderes System und treten dort belehrend auf, während das System, in dem sie sich befinden, eigentlich noch um Demokratisierung und Diversität kämpft. Aus einem System kommend, in dem das »Regie-Genie» an der Tagesordnung ist, Macht ungleich verteilt ist und Frauen, Menschen mit Migrationserfahrung oder Menschen mit Behinderungen lediglich als Randerscheinungen und in stereotypen Rollen akzeptiert werden, über die Freiheit der Künstler*innen und des Kunstmachens zu reden, kam mir vor, als würde ich mir selbst in die Tasche lügen.

\section{Übungsraume für Freiheit schaffen}

Das diverCITYLAB ist als ein Projekt entstanden, das die Kunst ins 21. Jahrhundert führen möchte. Wir arbeiten an einer Schnittstelle, die Theater und Bildung miteinander verbindet und durch ein Denken von Veränderung in beiden Bewegung erzeugt. Wir brauchen neue Strategien, diese beiden Felder zu demokratisieren, damit in der Zusammenarbeit eine demokratische Bindung entsteht. Daher profitiert diverCITYLAB sehr, wenn wir mit Schulen arbeiten, da die Arbeit für uns eine Möglichkeit zur Reflexion bietet.

Eine Beobachtung meinerseits ist, dass die Siebzehnjährigen häufig größere Strukturen und das Schulsystem thematisierten. Selbstzensur spielte eine große Rolle und immer wieder fiel in den Diskussionen: "Das darf man nicht sagen", "So kann man das doch nicht sagen dürfen «. Auch Gruppenzwang - genauer gesagt, die Frage wer sich äußern darf - beeinflusste die 
Gespräche. Die Schüler*innen hatten untereinander eine Hierarchie aufgebaut, aus der sie nicht mehr herauskamen.

Bei den Elf- bis Zwölfährigen waren Themen wie Mobbing und Außenseitertum in der Schule (bedingt durch vermeintlich mangelnde Fähigkeiten, äußeres Erscheinungsbild oder Migrationshintergrund) oder Geschlechterverhältnisse in der Familie viel wichtiger. Interessanterweise haben diese Schüler*innen sich nie die Frage gestellt, ob sie etwas offen äußern dürfen oder nicht, die Selbstzensur war kaum vorhanden (oder weniger offensichtlich).

Dort, wo Freiheit ausgeübt werden kann und soll, müssen Fantasien mehr Raum bekommen. Leider muss das Träumen in der Erwachsenenwelt erst wieder erlernt werden. Hier folge ich dem Sprichwort: Übung macht den Meister! Das Kind in uns müssen wir immer wieder neu entdecken und beleben. Wie Friedrich von Schiller schon sagte: »Der Mensch spielt nur, wo er in voller Bedeutung des Worts Mensch ist, und er ist nur da ganz Mensch, wo er spielt.«

Räume für Freiheit zu etablieren braucht auch bei diverCITYLAB Übung. Wir sind im Kunstbereich verankert, aber als eine offene Bildungsinstitution organisiert und versuchen, dem Lernen und Lehren ein anderes Gesicht $\mathrm{zu}$ geben. Oft scheitern wir daran, da der offene Zugang viele Diskussionen, Missverständnisse und Empfindlichkeiten mit sich bringt. Wir haben ständig mit Menschen zu tun und jede* $r$ benötigt etwas anderes, auch lernt jede* $r$ anders. Wir lernen jeden Tag von unseren Studierenden, jedes Jahr mit einer neuen Klasse, die Gruppendynamik ist immer neu und fordernd.

Mit den Konzepten Freiheit, künstlerische Freiheit und freie künstlerische Ausbildung haben wir als diverCITYLAB in unseren ersten Jahren viele Fehler gemacht und viele unserer anfänglichen Vorstellungen zerstört. So sehr, dass wir mit unserer ersten Klasse eine gemeinsame Gruppentherapie organisieren mussten, um in der Mini-Gesellschaft der Klasse wieder funktionsfähig zu werden.

Nachdem die ganze Klasse in einigen Sitzungen mit der Therapeutin eruiert hatte, was falsch lief, was ihre Probleme waren und wie es dazu kam, dass alles so eskalierte, hatten wir vom Team noch zusätzliche Sitzungen. Hier wurde uns klar, dass wir mit dem Begriff Freiheit zu leichtfertig umgegangen waren und erwachsene Menschen (die Studierenden waren zwischen 21 und 26) mit unserer Herangehensweise gewissermaßen in die Pubertät zurückgeführt hatten. Menschen, die in restriktiven Systemen sozialisiert 
worden sind, sei es in der Familie, in ihren Jobs oder in der Schule, die volle Freiheit für ihr Handeln und Denken anzubieten, hat zur Verweigerung der eigenen Disziplin geführt. Keine"r kam mit dem Begriff Freiheit zurecht, jede*r hatte ihn für sich anders interpretiert, und damit waren Zusammenstöße in der Gruppe vorprogrammiert. Wir waren frustriert, dass die von uns in Gang gesetzten Mechanismen nicht mehr kontrollierbar und vertretbar waren.

Die Sitzungen waren sehr erhellend. Wir suchten Maßnahmen zur Problemlösung und haben einen Workshop für die Studierenden angeboten. Mit der Künstlerin Anna De Carlo (unter anderem aktiv beim Zentrum für politische Schönheit und beim Social Muscle Club) wurden in Gruppen ${ }^{3}$ der Begriff Freiheit unter die Lupe genommen. Unterschiedliche Freiheitsbegriffe, vom individuellen über den kollektiven bis zum utopischen, wurden analysiert, um zum Schluss ein gemeinsames Bild von Freiheit und freiem Handeln zu schaffen.

Aus diesem Workshop heraus entstand eine Schein-Pop-up-Firma für Frieden auf der Welt mit dem Motto: "Ich kann den Frieden in der Welt nur dann schaffen, wenn ich mit mir und in kleinen alltäglichen Kämpfen Friedenswege finden kann.« Das Ganze endete mit einer künstlerisch-politischen Performance im öffentlichen Raum. Dabei fragten die Studierenden die Passant*innen: „Wollen Sie Weltfrieden?«, und anschließend: „Haben Sie immer Frieden mit Ihren Mitmenschen?« Es entstanden sehr persönliche Gespräche über die Konflikte der Passant"innen. Sie waren dankbar, dass ihnen jemand zuhörte, während die Studierenden den Wert davon erlebten, wie es ist, zuzuhören ohne zu beurteilen.

Nach diesem Workshop und der Performance hatten wir unser Ziel erreicht. Es herrschte wieder eine Diskussionskultur. Respekt für das Gegenüber war ein gemeinsamer Wunsch und die Freiheit wurde bewusst und mit viel Spaß umgesetzt. Nach dieser Erfahrung wurden gemeinsam mit den Studierenden einige Regeln in einem Leitfaden zusammengefasst, den die Studierenden am Anfang des Studiums unterschreiben. Wir wissen, dass wir es mit Individuen zu tun haben, daher sind die meisten Regeln veränder-

3 Zu dieser Zeit hatten wir zwei Klassen: den dritten Jahrgang, der die schon erwähnten großen Probleme hatte, und den ersten Jahrgang, der gerade angefangen hatte, sich mit Freiheit, sich selbst und Kunst zu beschäftigen. Die Teilnahme dieser Studierenden sahen wir nach den Erfahrungen als präventive Maßnahme. 
bar. Nur nicht jene, die uns als eine offene, lebendige Kunst- und Bildungssynthese auszeichnen.

"Spielt auf einer fairen und chancengleichen Spielwiese. Wir arbeiten zusammen. Und finden alles zusammen heraus.

Fragt immer und alles - wenn ihr wirklich Antworten braucht.

Trefft Entscheidungen und übernehmt die Verantwortung für eure Entscheidungen.

Wisst, was ihr wollt und macht es. Und dann bleibt aber offen für neue Entdeckungen und Möglichkeiten. Täuscht nichts vor. Seid ehrlich. Zu uns und zu euch selbst.

Erwartet nicht, geführt oder getragen zu werden. Aber begegnet den Anweisungen der Lehrenden und Regisseure mit Offenheit.

Bei untergriffigem und beschuldigendem Tonfall werden Fragen nicht beantwortet. Team, Lehrende und Studierende fühlen sich dann nicht mehr zuständig. Das gesamte Team von diverCITYLAB arbeitet unter prekären Umständen, dafür braucht es als Gegenleistung euren respektvollen Umgang. Wir sind für eine Gesellschaft, in der jegliche Art von Diskriminierung, Hass und Ausgrenzen keinen Platz haben. Ein Jahrgang ist ein Team, in dem jede_ $r$ für sich selbst verantwortlich ist. Das schließt jedoch nicht aus, dass man sich untereinander um einander kümmert. Dem Cedanken des Nehmenund-Cebens folgend bitten wir darum, dass diejenigen, die je nach Situation mehr nehmen müssen, von den anderen aufgefangen werden.«

Ein Raum, in dem Freiheit ausgeübt werden kann, ist weder im Theater noch in der Schule selbstverständlich, er muss erst gemeinsam geschaffen werden. Wir können nur aus dem Vergangenen lernen, so können wir es das nächste Mal besser machen. In Samuel Becketts Worten: „Ever tried. Ever failed. No matter. Try again. Fail again. Fail better.«

Es gibt großartige Lehrer*innen in den Schulen, die sich auch während ihrer Freizeit für künstlerisch-politische Schulprojekte einsetzen und alles tun, damit sie stattfinden. Wenn aber künstlerische (ich sehe das gesamte Projekt ebenso als philosophische und als politische Bildung) Projekte in den Schulen nur auf »Freiwilligkeit « basieren, ${ }^{4}$ und nicht als notwendige Maßnahmen für die persönliche soziale Entwicklung gesehen werden, nicht Teil

4 Siehe den Beitrag der Forschungsgruppe Freiheit in der Schule in diesem Band. 
des Lehrplans sind, bleibt die Ernsthaftigkeit eines solchen Projekts auf der Strecke.

Ein Raum, in dem Freiheit ausgeübt werden kann, muss durch einen Prozess erst gestaltet werden, er muss auch in Schulprojekten ein Übungsraum sein. Mit Theater die Welt zu erdenken, in der wir leben wollen, heißt auszuhandeln, in welcher Weise wir in einem Projekt den gemeinsamen Raum gestalten wollen, damit er tatsächlich ein Raum wird, der Grenzen infrage stellt und in dem Zukunft imaginiert werden kann. ${ }^{5}$

\section{Literatur}

Willenbacher, Sascha (2016). Der geschulte Blick. Bericht aus der Begleitforschung zum Projekt JUMP \& RUN, in: Willenbacher, Sascha/Schlie, Camilla (Hg.): »Eure Zwecke sind nicht unsre Zwecke«. Zur Kooperationspraxis zwischen Theater und Schule im Berliner Modellprojekt >JUMP \& RUN<, Bielefeld: transcript, 191-315.

5 Ich bedanke mich bei Nora Landkammer für die Anregungen beim Verfassen des Beitrags. 


\title{
Taktiken für eine verändernde Praxis Schnittstellen kritischer Kunstvermittlung und partizipativer Forschung
}

Renate Höllwart, Nora Landkammer, Elke Smodics

\begin{abstract}
Forschung heißt »Zusammenarbeit « und »Teamwork « und man macht sie »gemeinsam«: Diese Schlussfolgerungen einiger Schüler"innen ${ }^{1}$ im Rahmen des SparklingScience-Forschungsprojekts Making Democracy haben sich im Gedächtnis des Forschungsteams festgehakt. Die zitierten Jugendlichen hatten mit ihrer Feststellung, dass Forschen etwas Gemeinsames ist, den Kern partizipativer Forschung und kritischer Kunstvermittlung getroffen. Zugleich zeigt sich daran, dass eine Umdeutung stattfindet: Der Wissenschaftsbetrieb ist von individueller Autor"innenschaft und dem Kriterium der Exzellenz geprägt. Zu behaupten, dass sich Forschung über Kollektivität definiert, ist also eine Intervention in die etablierten Verständnisse von Wissenschaft. Wir wollen uns in diesem Beitrag mit solchen Deutungen und Umdeutungen von Wissen, Wissenschaft, Kunst und Kunstvermittlung beschäftigen.
\end{abstract}

Was sind Strategien und Methoden, vorhandenes Wissen in Austausch zu bringen und Platz für neue Perspektiven zu schaffen? Wie Räume schaffen, die ein Voneinander-Lernen ermöglichen? Was heißt gemeinsam forschen? Und welche Rolle hat die Anwendung künstlerischer Strategien in einem Forschungs- und Vermittlungsprozess wie diesem? Diesen und anderen Fragen möchten wir hier aus der Perspektive der kritischen Kunstvermittlung nachgehen. Zu Beginn skizzieren wir die Ausgangspunkte partizipative

1 Wiener Mittelschule und BORC antonkriegergasse, Reflexionsbögen zum Workshop am 30.1.2018. 
Forschung und kritische Kunstvermittlung und deren Schnittstellen. Am Beispiel von verschiedenen im Projekt angewandten Methoden wenden wir uns anschließend den Ansätzen und Strategien aus der Praxis zu, um diese $\mathrm{zu}$ konkretisieren und wiederum im Beziehungsgeflecht Forschung, Vermittlung und Kunst zu verorten.

\section{Partizipative Forschung}

Partizipative Forschung bezeichnet einen »Forschungsstil« und nicht ein bestimmtes Set von Methoden oder einen definierten Ansatz (Bergold/Thomas $2012,2)$ innerhalb der qualitativen Sozialforschung. Sie umfasst eine Bandbreite verschiedener Herangehensweisen mit dem gemeinsamen Kriterium, dass die Personen, die das beforschte Thema »etwas angeht « am Erkenntnisprozess entscheidend beteiligt sind. Den Kernpunkt partizipatorischer Forschung macht die Zusammenarbeit von Akteur*innen mit unterschiedlichen Wissens- und Erfahrungshintergründen aus. Bergold und Thomas grenzen entlang der Frage der Entscheidungskompetenz partizipative Forschung von anderen Beteiligungsformen wie Konsultation und Beratung ab: »Entweder sind die Menschen an Entscheidungen beteiligt und sind damit Forschungspartner/innen oder selbst Forschende, oder es findet keine partizipative Forschung statt.« (ebd., 7) Demnach sind die Beteiligten entweder Co-Forschende, die für eine bereits definierte Fragestellung an Erhebung und Auswertung beteiligt sind, oder Forschende, die eigene Fragen entwickeln und beforschen (Bragg/Fielding 2005, $207 \mathrm{f}$.). Die Rollen in dieser Zusammenarbeit sind von den Strukturen, in denen die Forschung stattfindet, geprägt. Partizipative Forschung lediglich als totale Autonomie der Forschungsgruppe zu begreifen, würde die strukturellen Begrenzungen durch Fördergeber und Institutionen vernachlässigen. Wesentlich für partizipative Forschung ist demnach, den Handlungsrahmen gegenüber den Mitforschenden transparent darzustellen, um innerhalb dessen Partizipation als tatsächliche entscheidende Mitgestaltung zu realisieren.

Bei Making Democracy, einem Projekt eingebettet in den Schul- und Universitätskontext, stand im Zentrum, dass die Schüler*innen innerhalb dieser Strukturen eigene Fragen und Wege ihrer Bearbeitung finden konnten. Die konkreten Inhalte, mit denen sich Making Democracy beschäftigte, die Wahl der Methoden sowie die Präsentationformen wurden von den 
Schüler*innen definiert. Während wir als Forschende und Vermittler*innen die Aufgabe der Beratung und Begleitung hatten, werteten wir zugleich den Prozess und die Ergebnisse in Hinblick auf unsere eigenen Fragestellungen aus. Dieses Ebenenmodell (vgl. Reason 1994) hat seine Tücken - denn die Partizipation wird dadurch selbst wieder zum Forschungsgegenstand (und damit auch die Schüler*innen), und statt einer kollektiven Forschung werden parallele Forschungsprozesse eingesetzt - gibt im Gegenzug jedoch Raum, den Forschungsprozess selbst zu gestalten, auch wenn die Interessen heterogen sind.

\section{Kritische Kunstvermittlung}

Kritische Kunstvermittlung als transdisziplinäre Bildungspraxis will durch das Arbeiten mit künstlerischen Strategien Räume für Imaginationen dessen eröffnen, was (noch) nicht da ist, aber sein könnte. Gleichzeitig werden Mittel an die Hand gegeben, um diese Vorstellungen auszuarbeiten und zu konkretisieren. Ein zentrales Anliegen ist die Ermöglichung von Teilhabe und Mitgestaltung durch alle Beteiligten (vgl. Smodics/Garnitschnig 2017, 14). Kritische Kunstvermittlung eröffnet Zwischenräume, in denen im Mittelpunkt steht, sich jenseits von Zuordnung und Zuschreibung darauf einzulassen, was zwischen Lernenden und zwischen Lehrenden und Lernenden entsteht und darauf, dass wir dies nicht schon vorher kennen können (Sternfeld 2017, 24). Dabei geht es nicht nur darum, dass alle Beteiligten mitreden und ihre Meinungen und Annahmen kundtun, sondern auch darum, wie über was verhandelt wird. Kritische Kunstvermittlung reflektiert Kategorien wie Geschlecht, Ethnizität und Klasse mit den Beteiligten (Mörsch 2013, 164), "sie positioniert sich z. B. anti-rassistisch und anti-sexistisch und setzt diese politische Haltung an die Stelle behaupteter Objektivität und verordneter Diplomatie« (Mörsch 2009, 21).

In der Auseinandersetzung mit Demokratie in unserem Projekt, konkret mit Denkmustern über Gleichheit, Freiheit und Solidarität war es nicht die Absicht, endgültige Antworten zu generieren, sondern vielmehr, scheinbar feststehende Bedeutungen zu befragen und in einem kollaborativen und komplexen Prozess der Aushandlung und Herstellung von Wissen in Bewegung zu bleiben. Dabei ist Wissensaneignung immer auch Aneignung von Wissen über sich selbst und über die eigene soziale Position - im Sinne der 
Suche nach Strategien und Methoden, die »zu einer Änderung des Selbstund/oder Weltverständnisses führen und sich darüber gleichzeitig auf ein Außen auswirken, und zwar strukturell und inhaltlich und für alle Beteiligten « (Garnitschnig 2019, 270). Denn in der Aneignung von Themenfeldern und in der Durchkreuzung von scheinbar feststehenden Annahmen über die Welt liegt das emanzipatorische Potenzial der kritischen Kunstvermittlung, die neben sozialwissenschaftlichen Ansätzen den methodischen Rahmen für die Arbeit im Projekt Making Democracy bildet.

\section{Praxen der gemeinsamen Wissensproduktion mit künstlerischen und sozialwissenschaftlichen Ansätzen initiieren}

Making Democracy setzte sich zum Ziel, kritische Kunstvermittlung und partizipative Forschung zu verknüpfen. Lassen sich diese beiden Ansatzpunkte auch separat beschreiben und sind in unterschiedlichen Diskursen verortet, haben sie in der praktischen Umsetzung viel miteinander zu tun. Die Schnittstellen sind in den Ansätzen selbst schon angelegt. Gemeinsames Forschen impliziert das Vermitteln einer fragenden Haltung und damit eine pädagogische Tätigkeit. Deutlich wird das besonders in Ansätzen, die das Forschen mit Schüler*innen als Bildungspraxis auffassen, beispielsweise wenn Duncan-Andrade und Morrell von »Youth Participatory Action Research as Critical Pedagogy« sprechen (Duncan-Andrade/Morrell 2008). Dass Jugendliche eine Rolle als Forschende einnehmen und selbst Untersuchungen durchführen, »the positioning of urban youth as critical researchers and transformative intellectuals«, ist ein wesentlicher Pfeiler der kritischen Pädagogik, die die Autoren entwerfen. Im Gegenzug setzen partizipative kritische Vermittlungsprojekte auf Ergebnisoffenheit und den Anspruch der gemeinsamen Wissensproduktion (und nicht nur Weitergabe), es wäre also naheliegend von einem forschenden Prozess zu sprechen. Gemeinsamer Bezugspunkt zwischen kritischer Vermittlung und partizipativer Forschung ist die Tradition kritischer Pädagogik. Nicht zufällig entstand ein wesentlicher Strang der partizipativen Aktionsforschung im Lateinamerika der 1970er Jahre mit Orlando Fals Borda, der Investigación-Acción Participativa als Teil einer wissenschaftlichen, pädagogischen und politischen Befreiungsbewegung definierte (Fals Borda 1987). Der meistrezipierte Exponent dieser Bewegung in der Pädagogik war Paulo Freire, der mit der Pädagogik der Unterdrückten 
(Freire 1973) die kritische Pädagogik international maßgeblich prägte. Wie gestaltet sich also diese Schnittstelle von kunstvermittelnder und forschender partizipativer Arbeit? Es folgen vier Einblicke anhand von Methoden und Prozessen im Projekt Making Democracy.

\section{Mit Bildern verlernen}

Was sind die Bezugspunkte zum Alltag der Beteiligten, wenn wir über Freiheit, Gleichheit und Solidarität sprechen? Wie eine gemeinsame Sprache finden? Über was und wie wird gesprochen?

Die Verwendung von Bildersets als Methode bildet den Ausgangspunkt dafür, Fragen in der Gruppe zu generieren und den inhaltlichen Rahmen des Forschungsprojekts zu skizzieren. »Bilder sind mit Lust verbunden. Sie generieren und reproduzieren Lust, indem sie Erinnerungen wachrufen, Fantasien erzeugen, Gewohnheiten zitieren und durchbrechen « (Sternfeld 2018, 181) und bergen das Potenzial für Umdeutung und Umwertung. Ohne konkrete Aufgabenstellung wählen zu Beginn alle Beteiligten jeweils eine Bildkarte aus, um Interessen, Geschmack, Begehren oder einfach nur Unverständnis mit dem Gezeigten in der Gruppe zu teilen. Und gerade weil wir umgeben sind von normierenden Bildern stellen wir an den Beginn des Vermittlungsprozesses alternative Bilder. Um den Raum zu öffnen »brauchen wir neue Bilder, alternative Entwürfe, die auf das verweisen, was da ist und übersehen wird und auch auf das, was sein könnte. Kämpferische Bilder, die Begrenzungen und Ungerechtigkeit ansprechen. Ironische Bilder, Persiflagen, Übertreibungen, Verfremdungen, Bilder, die dichotome Klassifizierungen und soziale Hierarchien irritieren « (Garnitschnig 2019, 272). Bilder von künstlerischen Produktionen und Protestbewegungen, die eindeutige Zuschreibungen oder normative Vorstellungen dekonstruieren und hegemoniale Narrative und Kategorisierungen durchkreuzen. Im gegenseitigen Zeigen und in einem gemeinsamen Ausverhandeln darüber, was die Bilder bedeuten könnten, entsteht eine Art Collage von Kommentaren, Wörtern, Fragen und Anliegen. Dabei geht es nicht um das Abfragen von kunsthistorischem oder geschichtlichem Vorwissen, sondern um die Eröffnung eines Sprachraumes, in dem Annahmen, Meinungen, Irritationen und spezifische Interessen miteinander in Beziehung gebracht werden. 
Ein Bild über eine Demonstration für das Wahlrecht für Migrant*innen aus den 1990er Jahren, das die ungleiche Verteilung von Informationen über das aktuelle Wahlrecht in Österreich zur Sprache bringt, verknüpft sich in der gemeinsamen Betrachtung mit dem zur Ikone für Demokratie gewordenen Gemälde Freiheit für das Volk von Eugene Delacroix von 1870, das aus dem Schulbuch vertraut ist. Historische Aufnahmen feministischer Bewegungen wie Mein Bauch gehört mir! von 1971 und eine Festnahme einer Suffragettin im Kampf um das Frauenwahlrecht in England von 1907 oder eine Aufnahme der Solidaritätsaktion »Eine Million Rosen für Angela Davis« zur Freilassung von Angela Davis 1971, gemeinsam mit aktuellen Bildern wie NO FUTURE von Banksy von 2010 oder Free Zehra Dogan, das die Freilassung der inhaftierten Künstlerin 2018 in Istanbul fordert, werden zur Matrize für Debatten über die Bedeutung von Freiheit, Gleichheit und Solidarität und die Wirkung von Bildern. Ein Beispiel dafür, wie stereotype Zuschreibungen verhandelbar werden, ist die Diskussion, die sich um ein Bild mit einer erhobenen Hand und/oder Faust als Symbol für Widerstand entwickelte. Mit diesem Bild, welches sich als Symbol für Protest, Kampf und Stärke etabliert hat, wurde Wissen über historische Ereignisse abgerufen und Übereinstimmung hergestellt - bis ein Stencil-Graffito von einer Frau an einer Hausmauer, bekleidet mit einem Sari, die Hand zur Faust erhoben, gewählt wurde. Plötzlich drängten sich andere machtvolle, normative Zuschreibungen in die Besprechung der Bilder - die Faust wurde umgedeutet in ein Symbol für Armut. Diese Irritation war zugleich der Ausgangspunkt für eine Diskussion darüber, woher unsere Bilder kommen und wie sehr stereotype Bilder unsere Annahmen formen und darüber, wie diese Annahmen die Vorstellungen der Welt durchkreuzen.

Die Diskussion über die Bildkarten war ein wesentlicher Schritt, um jeweils eigene Fragestellungen für die Forschung zu den zunächst abstrakten Begriffen Freiheit, Gleichheit und Solidarität zu entwickeln. Zugleich ermöglichte die Arbeit mit den Bildern als Einstieg in einen kollaborativen Forschungs- und Vermittlungsprozess auch uns selbst als Vermittlerinnen, uns aus Vorannahmen über das Wissen der Schüler*innen freizusetzen, eine Bildkarte auszuwählen und uns innerhalb der Gruppe inhaltlich zu positionieren. 


\section{Sich und andere befragen}

Wie wird eine/r zur Forscher*in? Wie den Raum und den Alltag untersuchen, in dem eine/r sich täglich aufhält? Wie in Normalitäten intervenieren?

Damit eine Frage an Mitschüler*innen vom Pausengespräch zum Forschungsprozess wird, ist eine Unterbrechung, ein neuer Blick auf die Situation nötig. Eine Methode einer Forschungsgruppe im ersten Schuljahr war, ihre Fragen zur Wahrnehmung von Freiheit und Unfreiheit im Schulgebäude mit künstlerischen Interventionen (von Borries et al. 2012, 126) an konkreten Orten im Schulgebäude zu verbinden. Sie verschlossen den Schuleingang mit Absperrband und bauten umgrenzte Zonen um bestimmte Bereiche im Schulhaus. An diesen Orten führten sie Befragungen zur Wahrnehmung von Freiheit in der Schule durch. Die Befragten sollten den Orten anhand einer Skala Eigenschaften zuweisen. Die Methode der Interventionen schuf ein Setting, das den Untersuchungsgegenstand sichtbar und besprechbar machte, und wirkte als Attraktion für die Befragten. Zugleich ist sie direkt nicht nur mit der Forschungsfrage, sondern mit dem darunterliegenden Interesse, Freiheiten in der Schule zu schaffen, verknüpft. Den Schuleingang abzusperren, war für die 12 bis 13-Jährigen selbst ein Akt, sich Freiheiten herauszunehmen.

\section{Gemeinsam handeln - Forschungswagen}

Was sind Tools des Versammelns, der Involvierung und Zeigens? Wie kann eine Vermittlung aussehen, die unterschiedliche Wissensformen ernst nimmt?

Der Forschungswagen ist eine mobile Installation, die als Display für die vielstimmigen Forschungsergebnisse beziehungsweise Bedeutungsproduktionen zu demokratischen Werten fungiert. Er ist Gefäß und Gefährt für die Sammlung der Recherchen, Beiträge und Ergebnisse der Schüler*innen der Unterstufe (1. Projektjahr). Bestehend aus mehreren Modulen, die auf die vielfältigen Forschungsergebnisse abgestimmt sind, bietet er Raum für aufbereitete Materialien. Er beinhaltet beispielsweise unterschiedliche Fächer und Boxen für das Fototagebuch, das die Recherche- und Entwicklungsphasen mit den beteiligten Akteur*innen festhält, Aufhängungen mit MP3-Playern für die Audiobeträge, wo eine klangliche Übersetzung von Ausschluss- 
mechanismen zu hören ist, eine Vorrichtung für einen Bildschirm, auf dem die Theaterperformances zu sehen sind, eine Schnur mit Wäscheklammern für die entstandenen Zines etc. (zu den Themen und Inhalten vgl. den Beitrag von Elke Rajal in diesem Band). Zudem ist der Forschungswagen auf ein fahrbares Gestell montiert und somit in mehrfacher Hinsicht beweglich.

Beim Forschungswagen handelt es sich um ein Wissensarchiv, das unterschiedliche Zugänge in der Auseinandersetzung mit dem Demokratiebegriff versammelt und gleichzeitig produziert dieses Tool abermals Wissen durch die Nutzer*innen. Es handelt sich also um einen bewegten Ort, wo verschiedene Praktiken entwickelt und ausprobiert werden können. Und um einen kollektiven Ort. In Projekten wie Making Democracy treffen verschiedene Menschen aus unterschiedlichen Wissenskontexten aufeinander und bilden temporär ein Kollektiv, dass in der Kennenlernphase "voneinander lernt«, was unter einem solidarischen Zusammenarbeiten in Auseinandersetzung mit politisch beweglichen Themenfeldern - wie in unserem Beispiel mit demokratischen Werten - verstanden werden kann. Von einem Kollektiv zu sprechen kann hier nicht heißen, dass alle das Gleiche wollen, sondern Gemeinsames aus den vielfältigen Interessen und Zielen entwickeln. Visuelle Darstellungssysteme wie der Forschungswagen, der die Recherchen, Ausdrucksformen und Zwischenergebnisse des Prozesses von und mit 50 Schüler*innen und dem Projektteam versammelt und von einer kollektiven Autor*innenschaft getragen ist, sind wesentlich für diese Dimension des Gemeinsamen. »Kollektives Handeln braucht ein Begehren nach dem Kollektiv, den Wunsch, etwas gemeinsam zu tun, eine Alternative zum individuellen Handeln zu haben.« (gender et alia 2013, 196)

\section{Glaube keinem Mindmap, das du nicht selbst gemacht hast ${ }^{2}$}

Wie einen offenen Forschungsprozess festhalten, der nicht-lineare Bewegungen des Nachdenkens zulässt? Und wie können wir uns gemeinsam an Diskussionen, Eindrücke und Fragen erinnern, die jeweils in einem bestimmten Moment der Zusammenarbeit entstehen?

2 In Anlehnung an den Slogan »Claube keiner Karte, die du nicht selbst gemacht hast« von Orangotango - Kollektiv für kritische Bildung und kreativen Protest, http://orangotango. info/projekte/kollektives-kartieren/ (letzter Zugriff: 11.8.2019). 
Eine häufig verwendete Methode in der Gestaltung von Gruppenprozessen ist die Methode des Mindmappings, welche auch in diesem Projekt in unterschiedlichen Formen und Funktionen angewandt wurde. Im ersten Jahr des Projekts wurden, im Sinne einer klassischen Methode des Mappings, die Assoziationen zu Begriffen wie Demokratie, Freiheit und Gleichheit versammelt und arrangiert und dabei Hierarchien von Begriffen durch neue Ordnungen unterlaufen und neue Zusammenhänge offengelegt.

Die Methode schafft durch die Visualisierung des Gesprächsprozesses die Möglichkeit, individuelle Bezüge und Wahrnehmungen aller Beteiligten zum Gegenstand und zum Ort des Bildungs- und Forschungsprozesses in eine kollektive Verständigung darüber zu überführen, worüber wir in welchen Bildern denken und in welchen Worten wir sprechen. Und sie geben uns als Vermittler*innen die Möglichkeit, Positionen offenzulegen, Inhalte einzubringen und sich zu diesen für alle nachvollziehbar zu verhalten. Im zweiten Jahr ermöglichte das Mapping der ausgewählten Bilder und selbstgemachten Polaroids, entlang der Themen Freiheit, Gleichheit und Solidarität geordnet, Beziehungen zwischen den gewählten Bildwelten und dem thematischen Projektrahmen durch gemeinsame Zuordnung herzustellen. Dies fungierte als »erster « Eintrag in ein gemeinsames, raumgreifendes und wachsendes Forschungstagebuch. Die entstandene »Karte« erlaubte, Materialien und Fragestellungen aus neuer Perspektive in den Blick zu nehmen, darin $\mathrm{zu}$ intervenieren, Inhalte $\mathrm{zu}$ überschreiben und schlicht, das bereits Gezeigte und Gesagte entlang des Forschungsprozesses nicht aus den Augen zu verlieren. Die Map war ein Tool zur Moderation und Begleitung des Forschungsprojekts, welches Vorgangsweisen aus Methoden des Mindmaps, Kartierens, Forschungstagebuch und schlicht des Protokollierens zusammenführte. Die Montage aus Bildern von künstlerischen Produktionen, Recherchebeispielen, angewandten künstlerischen und methodischen Strategien sowie Zwischenergebnissen aus Befragungen und Zeichnungen aus dem Comic-Workshop veranschaulichte die vielfältigen Ebenen der konkreten Themen, an denen Demokratie beforscht wurde, und eröffnete Einblicke in den unabgeschlossenen Prozess der Auseinandersetzung. 


\section{Mitten in der Dekonstruktion: Was wollen wir von Kunst, Pädagogik, Forschung?}

Nicht zuletzt hat das Überschneidungsfeld von kritischer Kunstvermittlung und partizipativer Forschung damit zu tun, dass beide Diskurse und Praxen sich daran abarbeiten, die Grenzen der Felder, in denen sie institutionell und diskursiv verortet sind, vehement infrage zu stellen, zu durchbrechen und zu erweitern. Partizipatorische Forschung versucht, die Grenzen dessen, was als Wissen und als Wissenschaft anerkannt ist, zu bearbeiten. Kritische Kunstvermittlung agiert zwischen dem Pädagogischen und dem Kunstfeld und hinterfragt, was als legitime Kunst gilt, wie das richtige Lernen definiert ist. Der Impuls dieser Dekonstruktion der Grenzen ist in der Beteiligung angelegt. Partizipative Wissenschaftler*innen, Künstler*innen oder Vermittler*innen beabsichtigen, ihre eigene Autorität und feldspezifische Rolle zu unterwandern. Wissenschaftler*innen wollen in partizipativen Forschungsprojekten zu Vermittler*innen werden, wenn sie sich als Berater"innen, als »facilitators bezeichnen (Bland/Atweh 2007; Brydon-Miller/Maguire 2009; Bragg/Fielding 2005). In partizipativen Vermittlungsprojekten wollen Vermittler*innen ihre Rolle als autorisierte Sprecher*innen im Kunstfeld dekonstruieren (Smodics/Sternfeld/trafo.K 2008).

Die Dekonstruktion der eigenen Rolle, die gerade in einem solchen transdisziplinär-partizipativen Unterfangen stattfindet, kann jedoch auch dazu führen, dass Lernen, Kunst und Wissenschaft als Gegenstand der Auseinandersetzung ungreifbar werden. Im Projekt Making Democracy stellten wir in der Reflexion fest, dass wir - gerade weil wir in Auseinandersetzung mit den Widerständen und Kritiken der Schüler*innen forcieren wollten, dass ihre Inhalte, ihre Interessen im Zentrum standen - unsere Rolle als Wissenschaftler*innen, als Pädagog*innen, unsere Erfahrung in künstlerischen Prozessen zunehmend zurückstellten. Hier stellt sich die Frage: Was bietet noch Motivation für einen Forschungsprozess, wenn die Teilnehmer*innen derart auf sich selbst zurückgeworfen sind? Wir wollen daher zum Abschluss darüber nachdenken, was wir an dieser transdisziplinären Schnittstelle von den Feldern, die zusammenkommen, wenn wir von kritischer Kunstvermittlung und/als partizipativer Forschung sprechen, einbringen wollen. 


\section{Niemand weiß schon genug: Lernen}

Wenn wir bereits die kritische Pädagogik Freires als gemeinsamen Bezugspunkt angeführt haben, wollen wir uns auch hier auf seine Texte beziehen. Freire beschreibt in Pädagogik der Hoffnung, was er mit der Pädagogik der Unterdrückten nicht gemeint hat - einer dieser Punkte ist, als Lehrende sich selbst abzuschaffen und auf das eigene Wissen zu verzichten: »Das Wissen [der Lernenden] anzuerkennen, von dem ich so viel spreche, um darüber hinauszugehen, kann niemals bedeuten, [...] dass der oder die Lehrende dabei stehen bleiben soll, bei diesem Wissen aus gelebter Erfahrung.« (Freire 2008, $110)^{3}$

Außerdem schreibt Freire: »[D]as Lesen der Welt darf nicht eines sein, das Akademiker den populären Klassen überstülpen. Aber dieses Lesen kann auch keine genügsame Übung der Lehrenden sein, die als Beweis ihres Respekts vor der populären Kultur gegenüber dem >Wissen aus gelebter Erfahrung schweigen und sich daran anpassen. Die dialektische und demokratische Position bedeutet im Gegenteil die Intervention der Intellektualität, als notwendige Bedingung für die Aufgabe. Und das ist keineswegs ein Verrat an der Demokratie [...] (ebd., 132 f.). Demgegenüber ist das Ziel, dass »das >Wissen aus gelebter Erfahrung notwendigerweise durch ein kritischeres, genaueres Wissen überwunden wird, ein Wissen auf das sie [die Lernenden] ein Recht haben« (ebd., 138).

Das »Gemeinsame« der Forschung und Vermittlung ist dann von Interesse, wenn etwas angeboten, eingebracht wird - neues Wissen, neue Methoden. Es gibt Wissen, das wir haben und ihnen geben wollen, das wir für relevant halten. Wir wollen einen Lernprozess und die Anerkennung, dass nicht alle schon das Wissen »mitbringen«, sondern dass Voneinander-Lernen Arbeit und Zeit braucht.

\section{Genau bleiben: Forschen}

Arjun Appadurai spricht von einem »Recht auf Forschung«, das allen zusteht (Appadurai 2006). Das trifft den Kern partizipativer Forschungsprojekte: Wenn wir von Forschung als Recht ausgehen, die Dinge herauszufinden, die

3 Übersetzung aus der spanischen Ausgabe für diese und die folgenden Zitate N. L. 
wir verstehen wollen, und unsere eigenen Schlüsse daraus zu ziehen, ist der Status als Forscher*innen nicht einer, der von der Akademia verliehen wird, sondern eine Bezeichnung für alle Menschen, die ihren Fragen systematisch auf den Grund gehen. Appadurai bezeichnet Forschung als »the capacity to make disciplined inquiries into those things we need to know, but do not know yet« (ebd., 167). Appadurais Definition ist auch deshalb für uns wichtig, weil sie klarmacht, dass Forschen kein beliebiges Anhäufen von Wissen ist. Er spricht von »disciplined inquires« oder »systematic forays« (ebd.), um über den bisherigen Wissenshorizont hinauszugehen. Für partizipative Forschungsprozesse ist an dieser Betonung wesentlich, dass sie Reflexivität in Bezug auf das Wissen und wo es herkommt impliziert. Es ist nötig, nicht bei der ersten, am einfachsten zugänglichen Informationsquelle stehenzubleiben, sondern zu fragen: Woher kommt dieses Wissen? Worauf beruht es? Methoden und Methodenreflexion heißt, auch bei den eigenen Erkenntnissen zu fragen, wie sie zustande gekommen sind: Wie sind die Antworten, die ich bekommen habe, vielleicht schon von der Art beeinflusst, wie ich die Frage gestellt habe? Welche Perspektiven sind vertreten, welche fehlen?

\section{Nicht allen alles recht machen: Arbeit mit Kunst und künstlerischen Strategien}

Die Arbeit mit künstlerischen Strategien und die Einbindung von künstlerischen Produktionen, die potenziell deutungsoffen sind, in einem Forschungsund Bildungsprozess öffnet Räume und kann Prozesse in Gang setzen, die Erfahrungen, Eindrücke und Vorstellungen neu verbinden, ohne dabei Widersprüche ausräumen zu müssen. Die Anwendung künstlerischer Strategien schafft einen Bezugsrahmen, in dem alternative Bilder und Sprachräume entstehen, die wiederum aus einer repräsentationskritischen Perspektive behandelt werden. Marianne Sorge betont für die kunstbasierte Forschung die Notwendigkeit, dass "Kunst nicht zur Illustration des Erforschten, sondern als eigenständige Denk- und Handlungsweise begriffen wird« (Sorge 2012, 124). Dies gilt ebenso für die Arbeit mit künstlerischen Strategien und Methoden in der Kunstvermittlung.

Man könnte sagen, kritische Kunstvermittlung eignet sich Strategien des Wilderns an (Sdun 2013, 278 f.). Die kollaborative Zusammenarbeit mit Künstler*innen und die Anwendung von künstlerischen Strategien ermöglichen in 
einem Forschungs- und Vermittlungsprojekt wie Making Democracy, offen zu bleiben für das Unerwartete jenseits von vorformulierten Lernzielen. Die Verschränkung der Methoden und Herangehensweisen aus den jeweiligen Feldern schafft einen Rahmen, der inhaltliche wie ästhetische Positionierungen aller Beteiligten zulässt, ohne Konflikte und Widersprüche immer und sofort harmonisieren zu müssen. Martin Krenn beschreibt soziale Kunstpraxen als jene Praxen, die durch ästhetische Methoden soziale Ordnung zwar anerkennen, sie jedoch verändern und in sie eingreifen wollen. "Kunst, die eine soziale Praxis verfolgt, muss nicht zwangsläufig demokratisch sein" (Krenn 2017, 251), vielmehr besteht sie auf ihre "politische Widerständigkeit, die sie aus ihrer ästhetischen Autonomie bezieht« (ebd., 254). Die Haltung der Widerständigkeit bewahrt sich kritische Kunstvermittlung, indem sie mit ästhetischen Formen und Rahmungen immer neu zur Dekonstruktion in Hinblick auf eine Veränderung der Verhältnisse ansetzt.

\section{Literatur}

Appadurai, Arjun (2006). The right to research, in: Globalisation, Societies and Education, Vol. 4(2), 167-177. https://doi.org/10.1080/14767720600750696. Bergold, Jarg/Thomas, Stefan (2012). Participatory Research Methods: A Methodological Approach in Motion, in: Forum Qualitative Sozialforschung/ Forum: Qualitative Social Research, Vol. 13(1), abrufbar unter: http:// www.qualitative-research.net/index.php/fqs/article/view/1801/3332 (letzter Zugriff: 3.8.2019).

Bland, Derek/Atweh, Bill (2007). Students as researchers: engaging students' voices in PAR, in: Educational Action Research, Vol. 15(3), 337-349. https:// doi.org/10.1080/09650790701514259.

Borries, Friedrich von/Hiller, Christian/Kerber, Daniel/Wegner, Friederike/ Wenzel, Anna-Lena (Hg.) (2012). Glossar der Interventionen. Annäherung an einen überverwendeten, aber unterbestimmten Begriff, Berlin: Merve, 126-133.

Bragg, Sara/Fielding, Michael (2005). It's an equal thing ... It's about achieving together: student voices and the possibility of a radical collegiality, in: Street, Hilary/Temperley, Julie (Hg.): Improving Schools Through Collaborative Enquiry, London/New York: Continuum, 105-135. 
Brydon-Miller, Mary/Maguire, Patricia (2009). Participatory action research: contributions to the development of practitioner inquiry in education, in: Educational Action Research, Vol. 17(1), 79-93. https://doi.org/10.1080/ 09650790802667469.

Duncan-Andrade, Jeffrey M./Morrell, Ernest (2008). Youth Participatory Action Research as Critical Pedagogy, in: The Art of Critical Pedagogy. Possibilities for Moving from Theory to Practice in Urban Schools, New York: Peter Lang AG, 105-131, abrufbar unter: https://www.jstor.org/sta ble/42979872 (letzter Zugriff: 1.8.2019).

Fals Borda, Orlando (1987). The Application of Participatory Action-Research in Latin America, in: International Sociology, Vol. 2(4), 329-347. https:// doi.org/10.1177/026858098700200401.

Freire, Paulo (2008). Pedagogía de la esperanza. Un reencuentro con la pedagogía del oprimido, Buenos Aires: Siglo Veintiuno.

Freire, Paulo (1973). Pädagogik der Unterdrückten. Bildung als Praxis der Freiheit. Reinbek bei Hamburg: Rowohlt.

Garnitschnig, Ines (2019). Sehen verlernen mit kritischer Kunstvermittlung und partizipativer qualitativer Forschung, in: Gottuck, Susanne/Grünheid, Irina/Mecheril, Paul/Wolter, Jan (Hg.): Sehen lernen und verlernen: Perspektiven pädagogischer Professionalisierung, Wiesbaden: Springer VS, 263-286. https://doi.org/10.1007/978-3-658-19496-3_12.

gender et alia - Fink, Dagmar/Lummerding, Susanne/Wiederspahn, Katja (2013). Kollektiv wie auch kollektive Praxis im kollektiven Arbeiten kontinuierlich neu zu re-artikulieren: eine Herausforderung, in: Fink, Dagmar/Krondorfer, Birge/Prokop, Sabine/Brunner, Claudia (Hg.): Prekarität und Freiheit? Feministische Wissenschaft, Kulturkritik und Selbstorganisation, Münster: Westfälisches Dampfboot, 190-199.

Krenn, Martin (2017). Zur Demokratisierung der Kunst/On the Democratization of Art, in: Krenn, Martin/Morawek, Katharina (Hg.): Urban Citizenship. Zur Demokratisierung der Demokratie/Democratising Democracy, Wien: Verlag für moderne Kunst, 239-281.

Mörsch, Carmen (2013). Kritische Kunstvermittlung, in: ARGE Schnittpunkt (Hg.): Handbuch. Ausstellungstheorie und -praxis, Wien/Köln/Weimar: Böhlau, 164.

Reason, Peter (1994). Three Approaches to Participative Inquiry, in: Denzin, Norman K./Lincoln, Yvonna S. (Hg.): Handbook of Qualitative Research, Thousand Oaks: Sage, 324-339. 
Sdun, Nora (2013). Wildern, in: Reichensperger, Petra (Hg.): Begriffe des Ausstellens (von A bis Z)/Terms of Exhibiting (from A to Z), Berlin: Sternberg Press, 278-279.

Smodics, Elke/Garnitschnig, Ines (2017). Das Projekt »Strategien für Zwischenräume. Neue Formate des Ver_Lernens in der Migrationsgesellschaft«, in: Büro trafo.K (Hg.): Schulheft 165, Innsbruck: Studienverlag, 12-20.

Smodics, Elke/Sternfeld, Nora/Büro trafo.K (2008). Kunstvermittlung als Handlungsraum. Definitionsmacht und Wissensproduktion am Beispiel einer Ausstellung von Jugendlichen für Jugendliche, in: Oldenburgische Landschaft (Hg.): Transfer 07 - Neue Wege in der Museumspädagogik und Kunstvermittlung, Oldenburg: Isensee, 39-57.

Sorge, Marianne (2012). Von uns an euch: wir wollen gehört werden. Rundgang durch ein ambivalentes Verhältnis von Wissenschaftlichkeit und künstlerischer/kunstbasierter Forschung (nicht nur) an der Akademie der bildenden Künste, in: Ortner, Rosemarie (Hg.): exploring differences. Zur Vermittlung von Forschung und Bildung in pädagogischer Praxis, Wien: Löcker, 121-132.

Sternfeld, Nora (2017). Was wächst in Zwischenräumen? Ein theoretischer Begriff im Hinblick auf die Praxis, in: Büro trafo.K (Hg.): Schulheft 165, Innsbruck: Studienverlag, 21-25.

Sternfeld, Nora (2018). Sich etwas zeigen, das es noch nicht gibt. Gedanken zur Repräsentationspraxis von Büro trafo.K, in: dies.: Das radikaldemokratische Museum, Berlin/Boston: De Gruyter, 181-223. 



\section{Freiheit in der Schule - oder haben wir die überhaupt? Eine Selbstbefragung}

Forschungsgruppe Freiheit in der Schule, 7B antonkriegergasse

F: Warum müssen wir alle nach einem Raster funktionieren, aus dem wir nicht rausfallen dürfen?

A: Weil es die einfachste Lösung ist. Fair und gleich gibt es bei uns nicht. Es ist nur eine Illusion.

F: Wieso dürfen alte Säcke bestimmen, was wir lernen, obwohl sie seit Jahren keine Schule mehr betreten haben?

A: Weil wir es erlauben. Sie sagen, was wir machen sollen und wir machen es einfach. Wir wehren uns nicht, wir sagen nicht: Stopp, wir wollen das nicht mehr machen! Sie sind die Erwachsenen, sie kennen sich besser aus und wir sind das eben nicht.

F: Spielt es eine Rolle in der Schule, woher du kommst, woher deine Eltern kommen, was für eine Staatsbürgerschaft du hast? Ist das Schulsystem inklusiv und tolerant genug? Gibt's Ärger? Gibt's Konflikte?

A: [Schweigen]

F: Warum wurde die Zentralmatura eingeführt?

A: Die Zentralmatura wurde eingeführt, um jedem Schüler und jeder Schülerin die gleichen Möglichkeiten zu geben. Die Grundidee ist eigentlich recht gut, aber dadurch, dass jeder Mensch unterschiedlich ist, geht sie nicht auf jeden individuell ein, sondern nur auf ein Schema, wo nicht jeder reinpasst. Und dadurch gibt es auch die Probleme, die so lange schon bestritten werden.

F: Warum müssen wir in den Ferien so viel für die Schule machen, obwohl doch eigentlich Schulferien sind? 
A: Ja, unsere VWA [Vorwissenschaftliche Arbeit] müssen wir ja auch in den Ferien schreiben und das nervt, weil das ganze Jahr über ist man mit den Gedanken ja in der Schule. Man existiert dann eigentlich nur mehr für die Schule und das ist falsch.

\section{F: Welcher Arsch hat den Lehrplan erfunden?}

A: Also meiner Meinung nach existiert kein Lehrplan. Er ist nur eine sehr einfache Ausrede für Lehrerinnen und Lehrer, um sagen zu können: »Uh, dieses Thema, das kann ich jetzt nicht mehr durchnehmen.« Oder: »Dieses Thema ist nicht im Lehrplan drinnen für dieses Jahr. «Aber in Wirklichkeit ist es mehr eine Ausrede, um sagen zu können: »Dieses Thema kam die letzten Jahre nicht zur Matura, folglich nehme ich das dann doch nicht durch, weil dieses Thema kam viel öfter zur Matura, dafür braucht ihr viel mehr Zeit zum Üben.«Also meines Erachtens gibt es keinen Lehrplan.

F: Wie ist das Verhältnis zu Lehrerinnen und Lehrern?

A: Das Verhältnis zu Lehrerinnen und Lehrern ist bei uns in der Schule eigentlich sehr gut. Ich kann nur berichten, die meisten Lehrerinnen und Lehrer werden respektvoll behandelt, wenn sie die Schülerinnen und Schüler natürlich auch respektvoll behandeln und den Unterricht gut und interessant halten.

\section{F: Was hältst du von freiwilligen Projekten in der Schule?}

A: Es kommt darauf an. Wenn das Projekt tatsächlich freiwillig ist, dann ist es meiner Meinung nach sehr cool, aber viele Projekte sind nicht freiwillig, sondern »freiwillig«. Mit anderen Worten, man wird gefragt: »Wollt ihr das machen?«, falls man überhaupt gefragt wird. Oder du kommst zum Beispiel zufällig in eine neue Klasse und du bist sofort eingeteilt in einem Projekt. Meiner Meinung nach ist ein freiwilliges nur dann ein freiwilliges Projekt, wenn jedes Mitglied sagt: »Dieses Projekt unterstütze ich, möchte ich machen und habe ich mir auch freiwillig ausgesucht.« Nicht: »Es wurde mir aufgezwungen.«

Aus dem gemeinsam mit Schauspielstudent*innen von diverCITYLAB erstellten Video für die Abschlusspräsentation, Mai 2019. 


\section{Making Democracy in der Schule}

\section{Bildstrecke}

Konzept: Renate Höllwart, Elke Smodics

Eigentlich geht's uns gut

Wir bringen alles unter einen Hut

Eigentlich geht's uns gut

Wir bringen alles unter einen Hut

Der Hut des Lebens

Hut Hut Hut Hut Hut Hut Hut... des Lebens

$8 x$

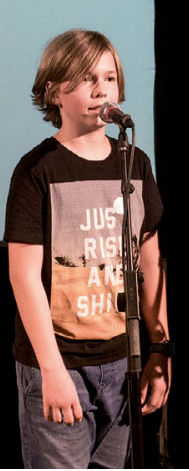





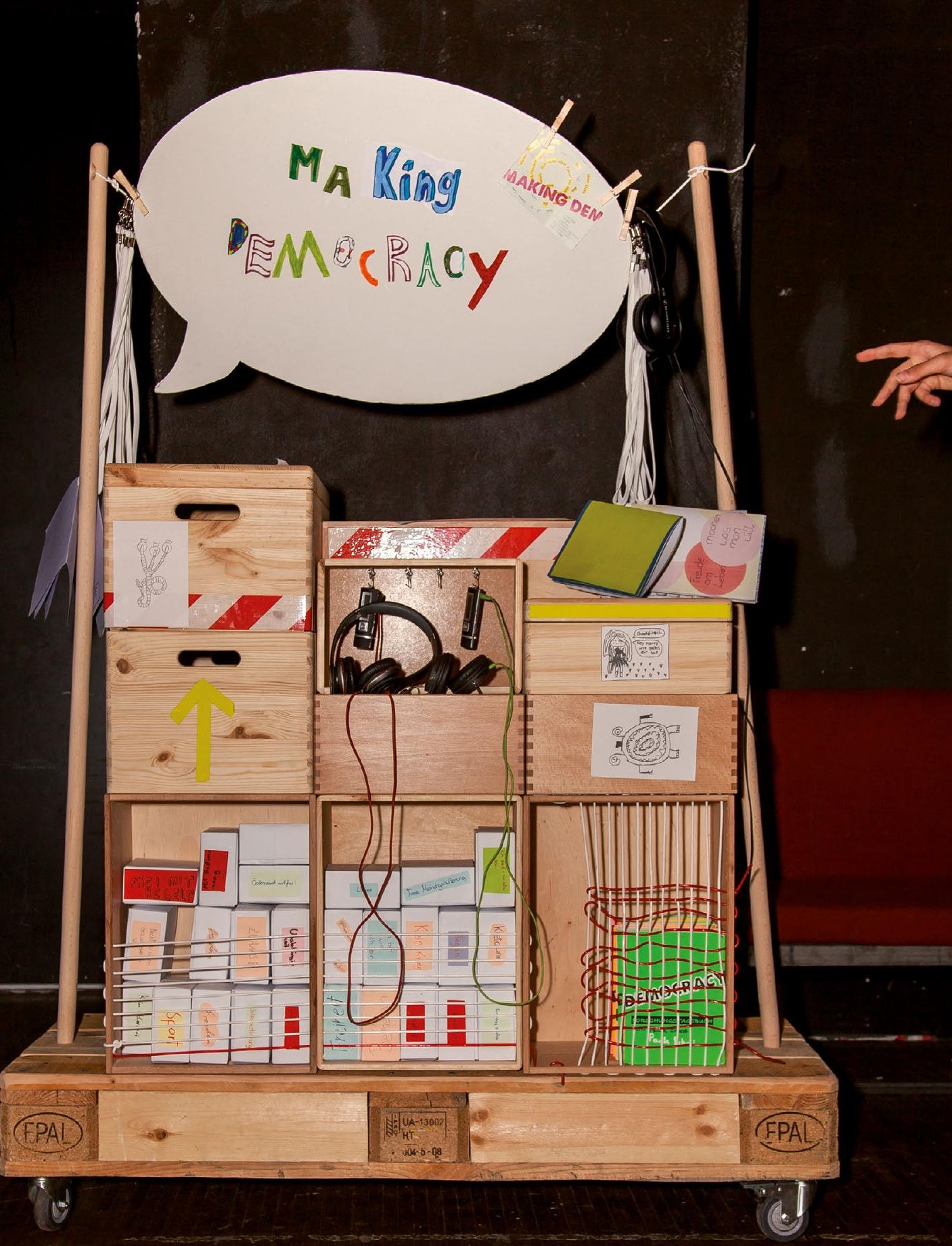




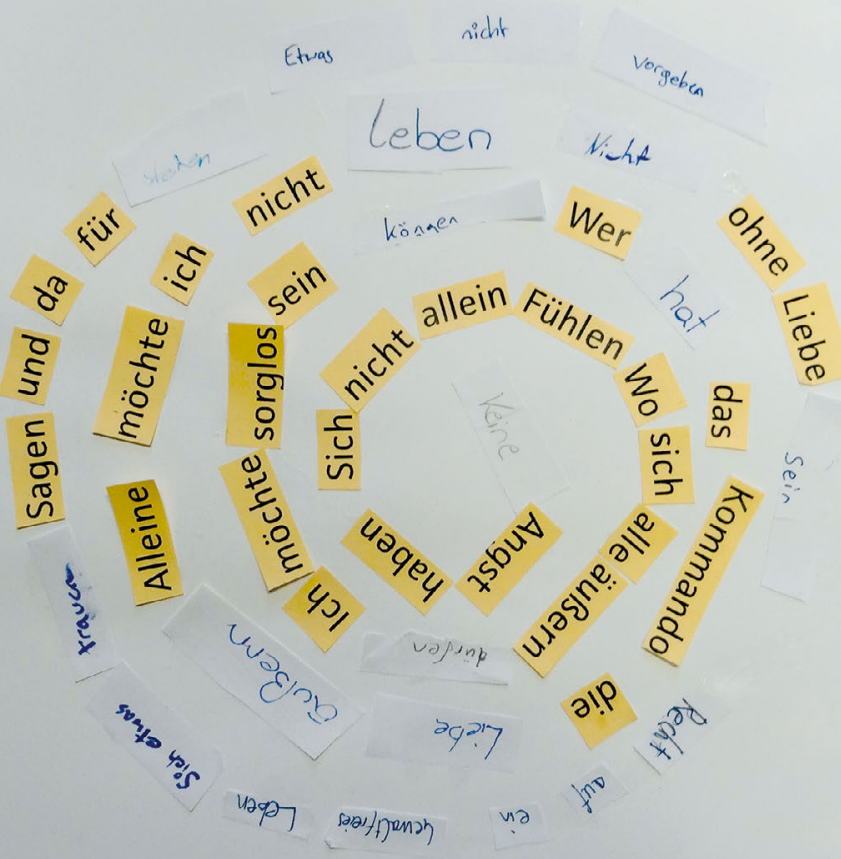


the

al

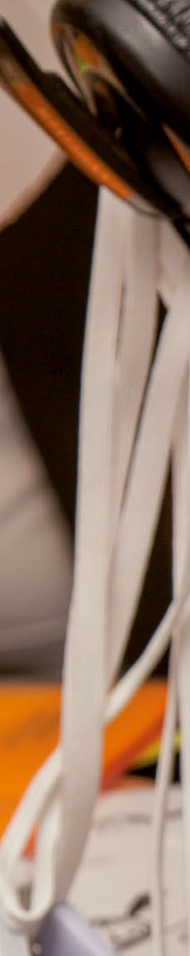

$+\infty$

$f^{2}$

N

1

4

$\frac{1}{6}-\frac{1}{2}$

1

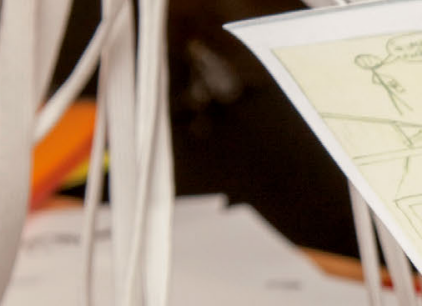

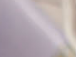

10

13

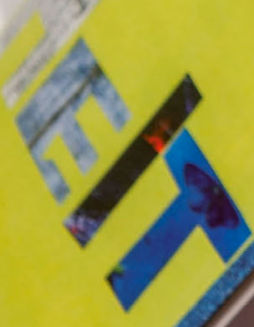

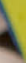

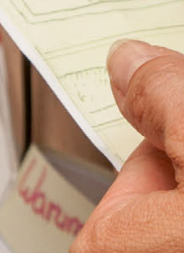

.
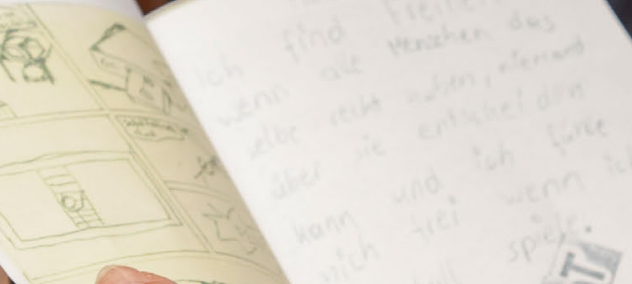


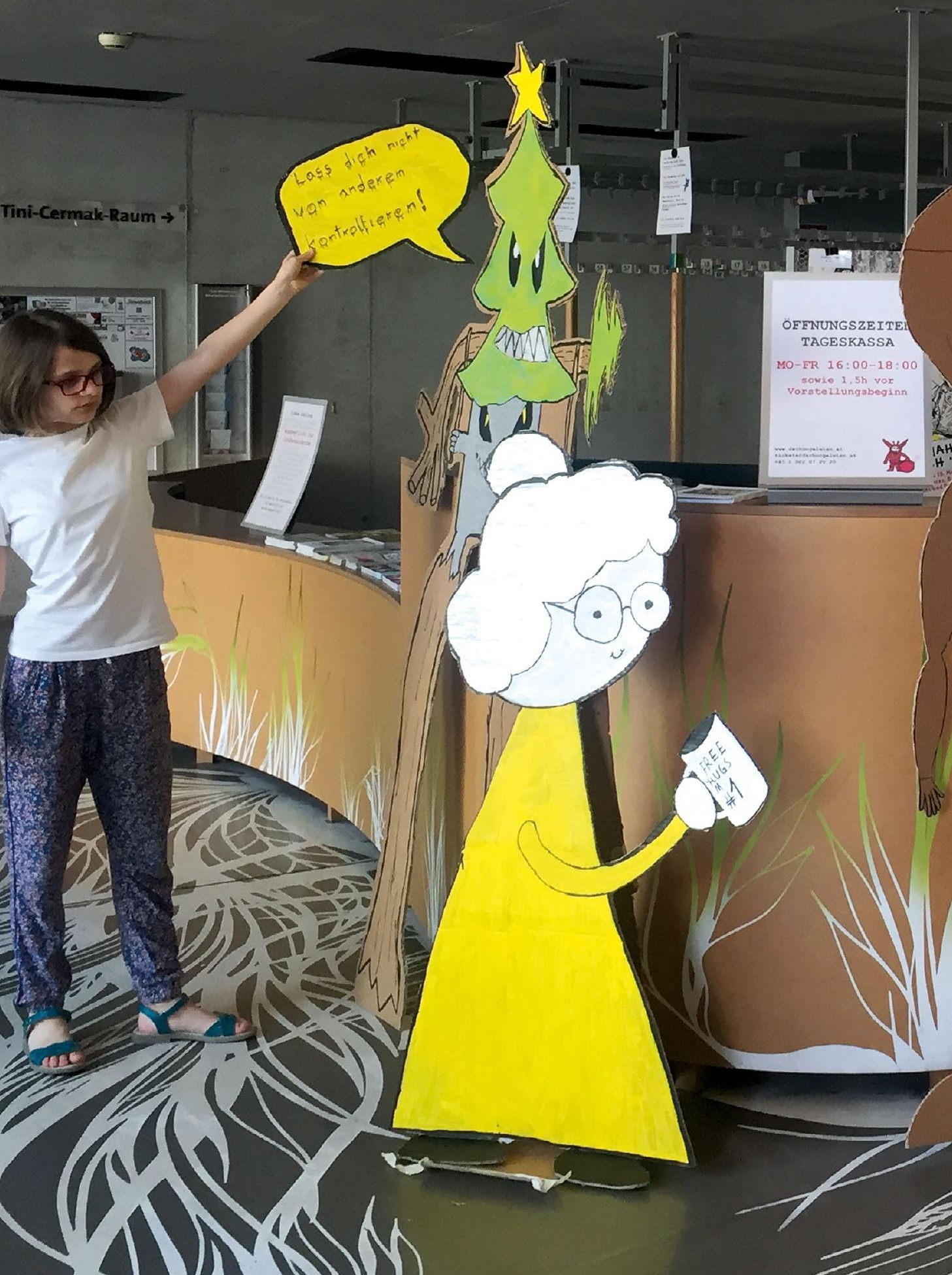




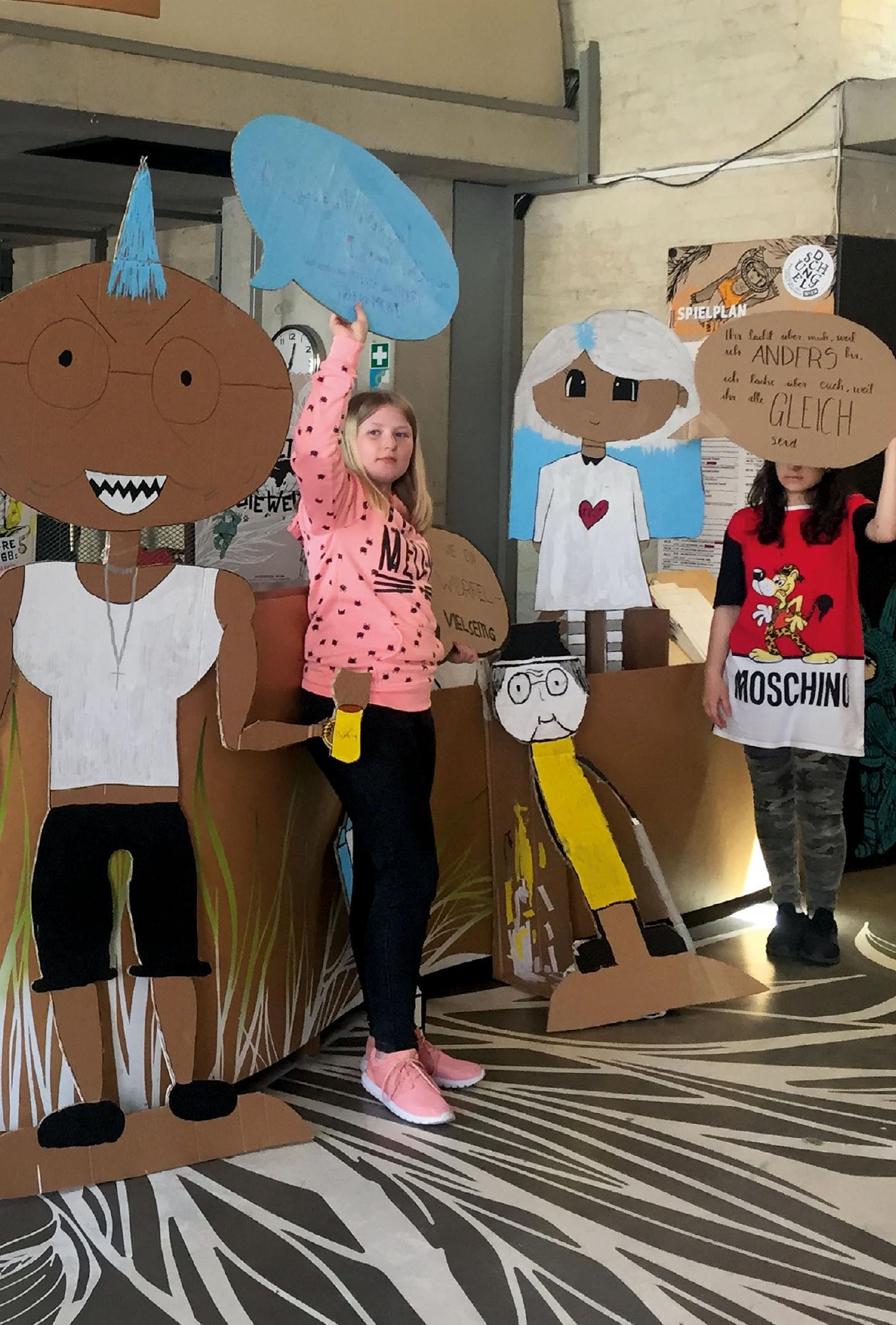




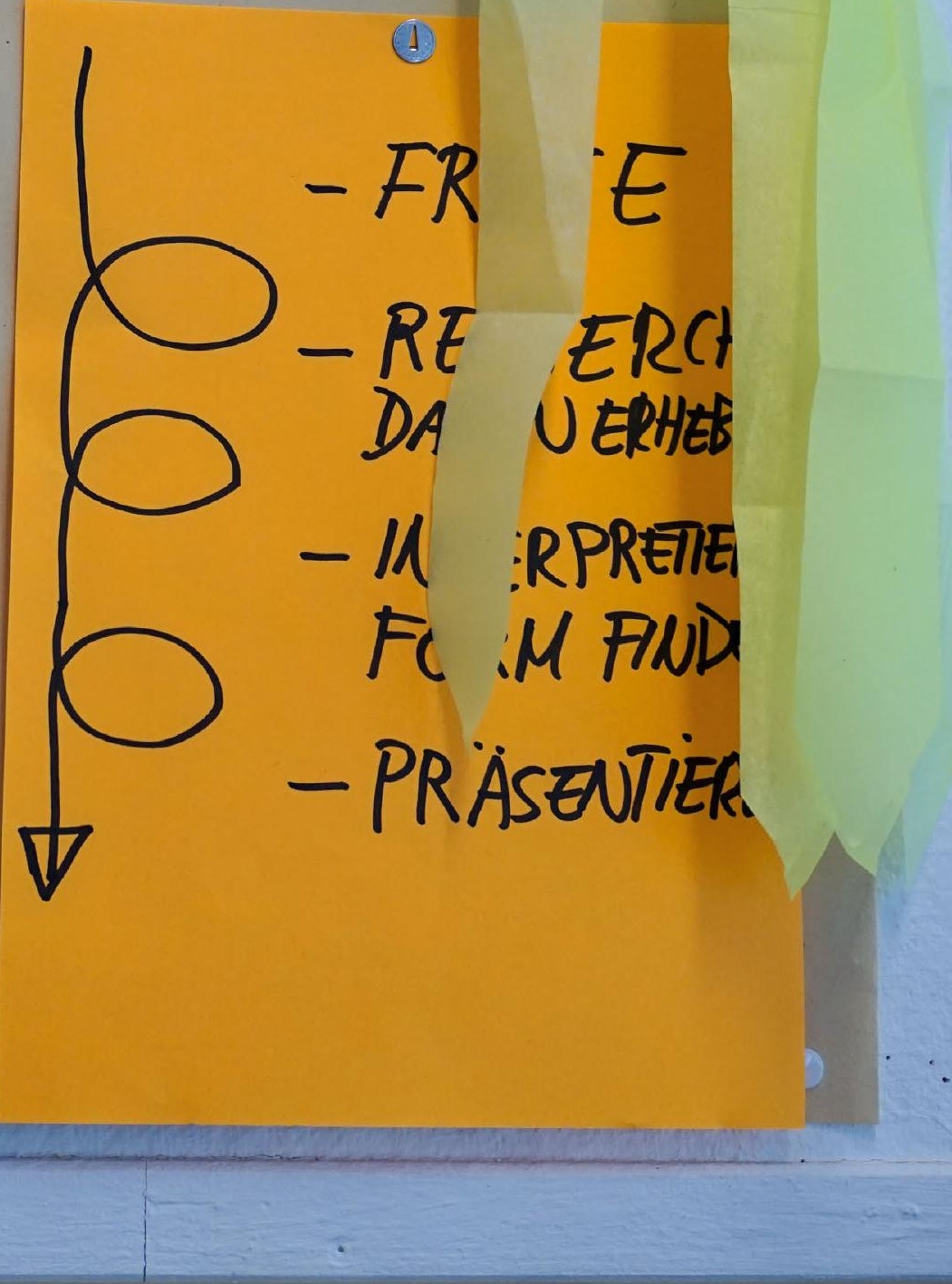





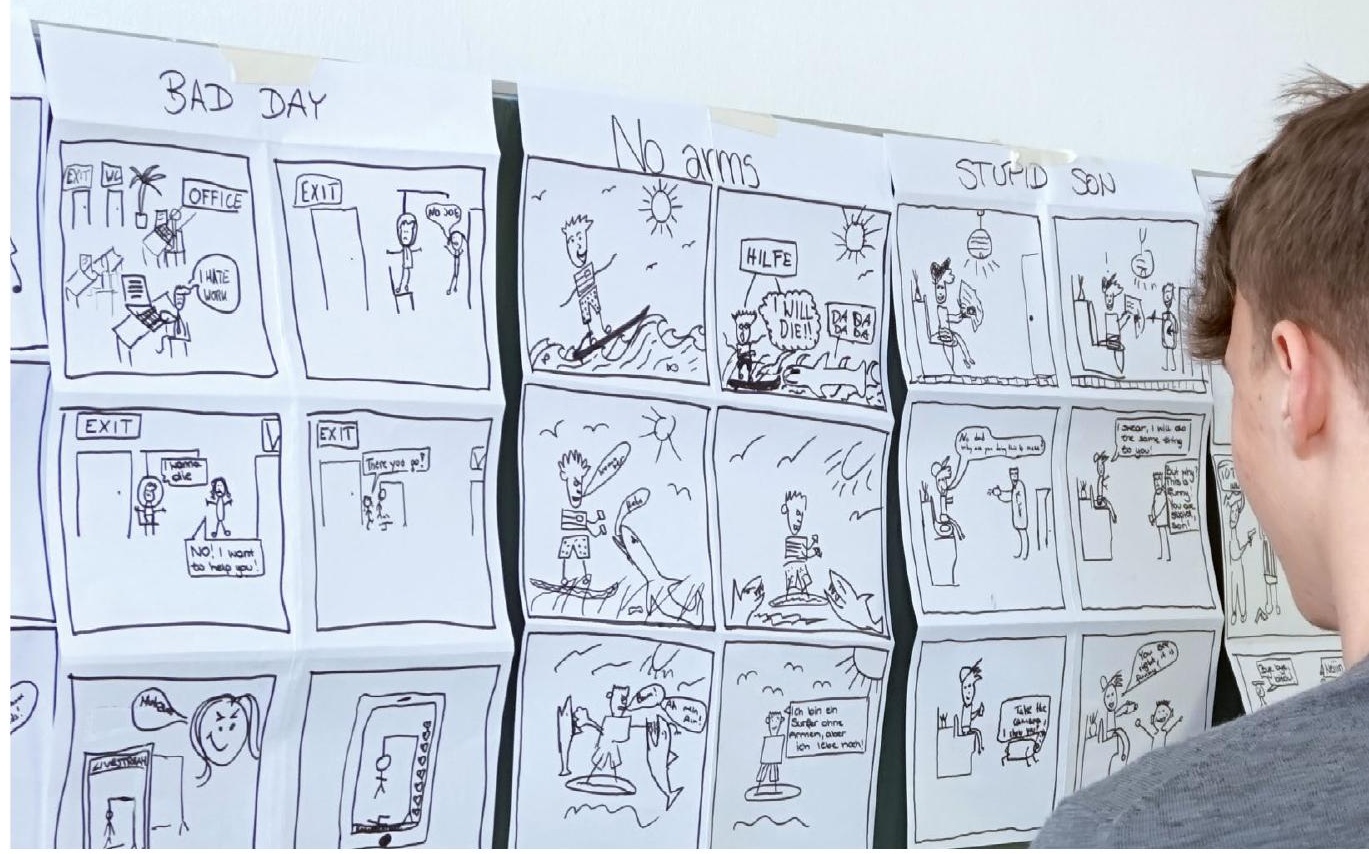




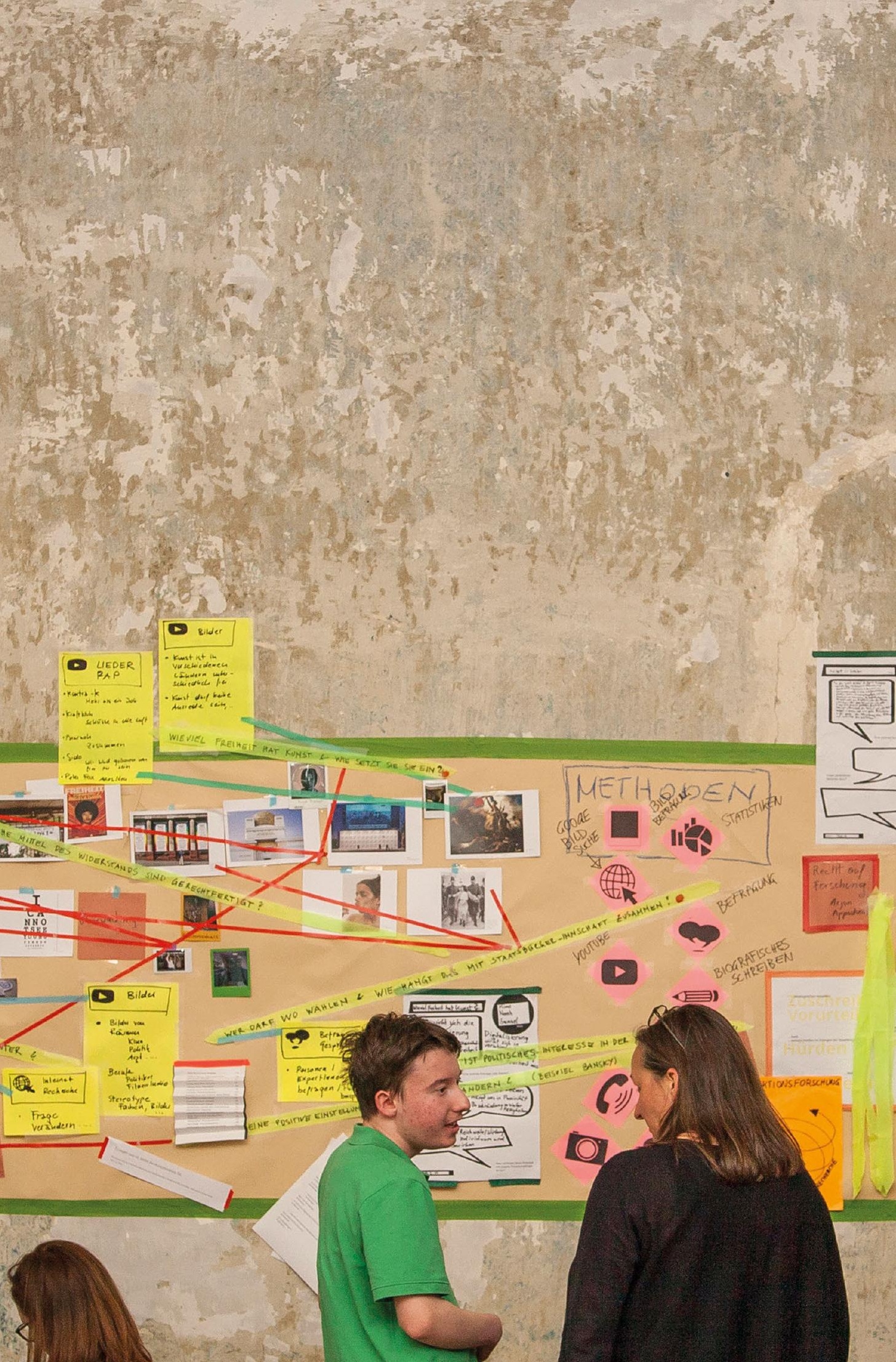




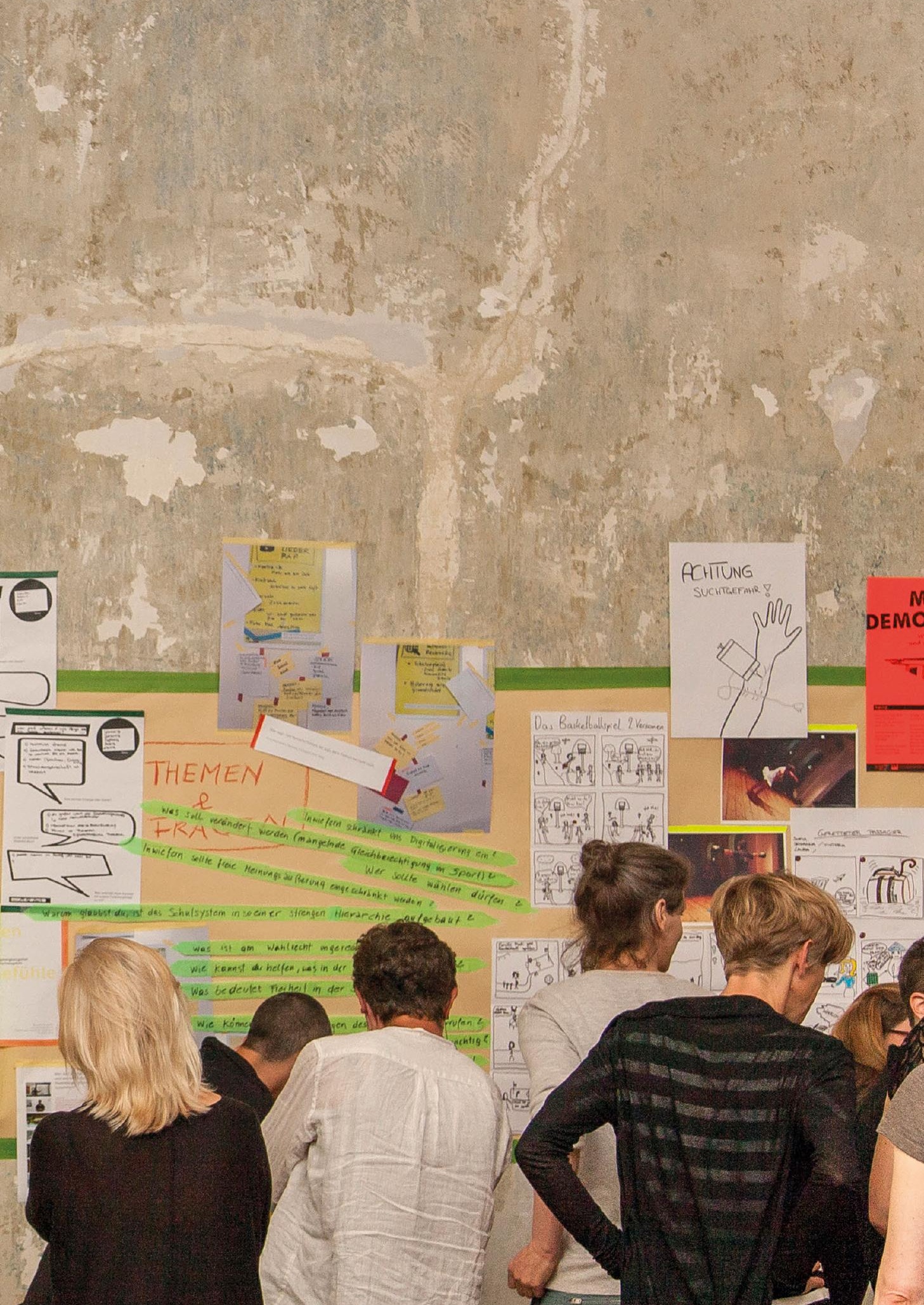



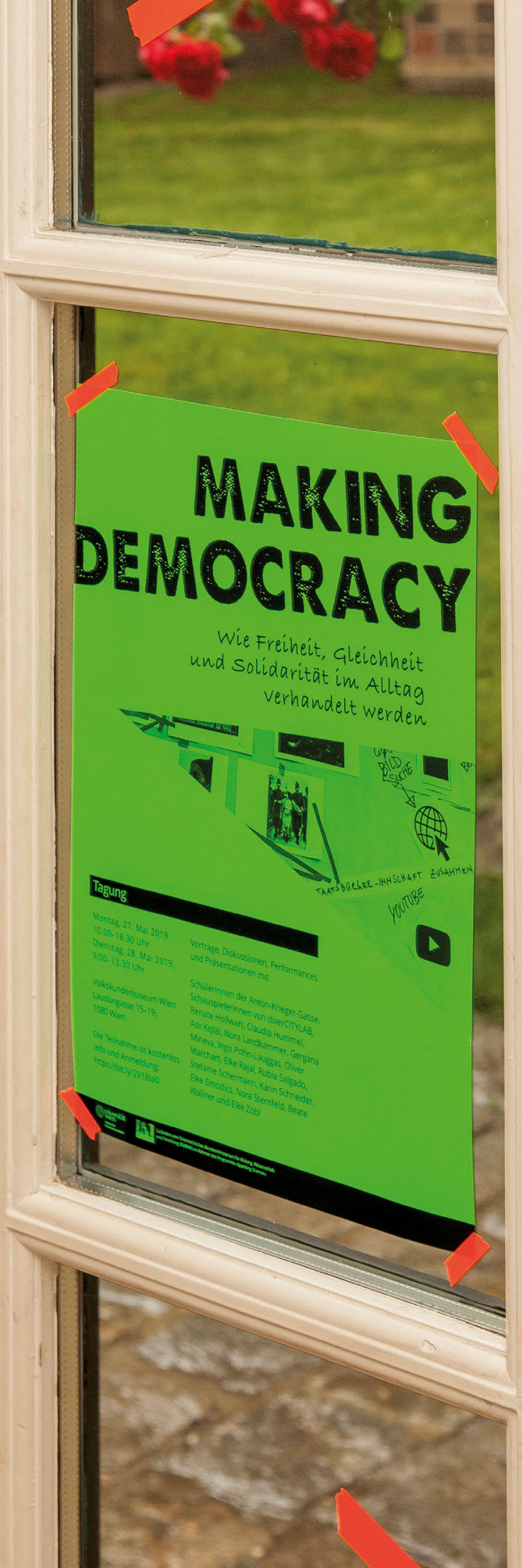


\section{Bildinformationen}

S. 103: akg is making democracy, Dschungel Wien, Schüler"innen der $3 \mathrm{~A}$ und der 3B, Foto: Sandra Kosel

S. 104: akg is making democracy, Dschungel Wien, Forschungswagen, Schü-

ler*innen der 3A und der 3B, Foto: Sandra Kosel

S. 105: akg is making democracy, Dschungel Wien, Forschungswagen, Schü-

ler*innen der 3A und der 3B, Foto: Sandra Kosel

S. 106: Kick off, Schüler*innen der $3 A$ und der $3 B$, Foto: trafo.K

S. 107: akg is making democracy, Dschungel Wien, Forschungswagen, Schü-

ler*innen der 3A und der 3B, Foto: Sandra Kosel

S. $108 \mathrm{f}$.: akg is making democracy, Dschungel Wien, Comics, Schüler*innen der 3A und der 3B, Foto: Sandra Kosel

S. 110: Forschungsprozess, Schüler*innen der 3A und der 3B, Foto: Elke Rajal

S. 111: Tagung, Volkskundemuseum, Präsentation, Schüler*innen der 3A und der 3B, Foto: Sandra Kosel

S. 112: Exkursion Belvedere 21, Ausstellung Der Wert der Freiheit mit Katja Stecher, Schüler*innen der $7 \mathrm{~B}$, Foto: trafo.K

S. 113: Forschungsprozess, Schüler*innen der $7 \mathrm{~B}$, Foto: trafo.K

S. 114: Workshop mit Ka Schmitz, Schüler*innen der 7B, Foto: trafo.K

S. 115: Workshop mit Ka Schmitz, Schüler*innen der 7B, Foto: trafo.K

S. $116 \mathrm{f}$.: Tagung, Präsentation, Schüler*innen der 7B, Foto: Sandra Kosel

S. 118: Tagung, Volkskundemuseum, Foto: Sandra Kosel 



\title{
Orte der Liebe und der Ausgrenzung Re-Lektüren einer Forschungsgruppe
}

\author{
Karin Schneider
}

\begin{abstract}
In diesem Beitrag beschreibe ich Erlebnisse und Denkprozesse, die ich in einer kleinen Forschungsgruppe im Rahmen des Projekts Tricks of the Trade. Feldforschung mit Schüler*innen (2008 bis 2010) machte ${ }^{1}$. Ich möchte diese partizipative Forschung im Hinblick aufein bestimmtes Handlungs-und Methodenrepertoire reflektieren, nämlich das der Kunstvermittlung. Im Zusammenhang eines sozialwissenschaftlichen Forschungsprojekts mit Jugendlichen verstehe ich darunter nicht das Arbeiten mit oder über Kunst und auch nicht das direkte Verwenden kunstbasierter Praxen. Vielmehr bemerkte ich von mir oft unbewusst eingesetzte Zuhör-und Frageweisen oder Vorschläge zur gemeinsamen Reflexion in den Gesprächssituationen mit den Jugendlichen. Diese Haltungen sind davon geprägt, wie ich mich in der Vermittlungsarbeit in Ausstellungen mit Jugendlichen den Irritationserfahrungen von moderner Kunst und Gegenwartskunst nähere. Ich möchte diskutieren, wie inmitten dieser Prozesse Ausschlüsse hergestellt wurden. Die von mir eingesetzten Praxen der Gesprächsführung bildeten den Rahmen für diese Ausschlüsse und waren gleichzeitig die Basis, um sie zu bearbeiten.
\end{abstract}

\footnotetext{
1 http://research.science.co.at/projekte/tricks-trade (letzter Zugriff: 16.6.2019).
} 


\section{Partizipative Haltungen und Verfahren}

Meine Überlegungen in diesem Text speisen sich aus unterschiedlichen Veröffentlichungen der beiden von Sparkling Science geförderten und von Veronika Wöhrer geleiteten Projekte Tricks of the Trade und Grenzgänge ${ }^{2}$. Allerdings beziehe ich mich auf jene eigenen Erfahrungen, in welche ich explizit Haltungen und Gesprächspraxen aus dem Vermittlungskontext einfließen ließ - das heißt, ich werde auf Beispiele meiner ersten Forschungsgruppe aus dem ersten der beiden Projekte zurückgreifen. Ich spreche hier von Haltungen, da es mir weniger um die Ausformulierung methodischer Arbeitsweisen, sondern um bestimmte Formen der Gesprächsführung geht. Diese sind an Praxen geschult, die ich im Museum anwende, wenn ich mich mit Jugendlichen den Irritationen nähere, die moderne Kunst oder Gegenwartskunst auslösen können. Es ist dies eine bestimmte Form des neugierigen Zuhörens, des direkten, unmittelbaren Zeigens eines genuinen Interesses an den Lebenswelten und Gedanken der Jugendlichen und gleichzeitig der Lust, deren selbstverständliche Annahmen durcheinander zu bringen beziehungsweise diese eigene Lust daran auf die Jugendlichen dadurch zu übertragen, dass mensch sich selbst bezweifelt. ${ }^{3}$

Eine (durchaus noch untersuchenswerte) unterstützende Anordnung für einen solchen Prozess ist das, was Andrea Hubin und ich einmal in sehr

2 Analysen aus beiden Projekten wurden in dem von Veronika Wöhrer, Doris Arztmann, Teresa Wintersteller, Doris Harrasser und mir verfassten Band Partizipative Aktionsforschung mit Kindern und Jugendlichen. Von Schulsprachen, Liebesorten und anderen Forschungsdingen veröffentlicht (Wöhrer/Arztmann/Wintersteller/Harrasser/Schneider 2017). Die methodischen Überlegungen wurden in einem Praxishandbuch Sozialwissenschaftliches Forschen mit Kindern und Jugendlichen zuerst im Eigenverlag bei Science Communication Research veröffentlicht und dann in stark überarbeiteter und erweiterter Form 2018 bei Beltz Juventa. Für das Projekt Tricks of the Trade gestalteten wir gemeinsam mit co-forschenden Jugendlichen eine Webseite, auf der sich sowohl wissenschaftliche Texte, Praxisreflexionen, eine Methodenbox (Trickkiste) und vor allem Reflexionen und Beiträge der Schüler*innen finden: www.tricksofthetradeproject.info (letzter Zugriff: 16.6.2019).

3 Ihre theoriebezogene Referenz haben diese Formen des Vermittlungswissens zumindest in meinem Fall vor allem in den Schriften der Kunstvermittlerin Eva Sturm; in einem ihrer zahlreichen Vorträgen zu Kunstvermittlung »von Kunst aus« beschreibt sie den Arbeitsplatz von Vermittler “innen als einen »[...] Platz, der [...] die Fähigkeit verlangt, zu schweigen und zuzuhören, Situationen zu drehen, zwischen den Worten wahrzunehmen, Sprache(n) und Körper nicht außer acht zu lassen, sich selbst/die eigenen Worte zu relativieren« (Sturm 2012, 5). 
freier Anlehnung an Bertolt Brecht die »dritte Sache « genannt haben (Hubin/ Schneider 2017, 453-462). Es ist Teil dieser Haltungen, dass Gespräche an ein Ding rückgebunden werden, welches allerdings selbst rätselhaft ist oder über die Betrachtung rätselhaft wird: Im Falle der Kunstvermittlung im Museum sind es Kunstwerke, die mithelfen, die Assoziationen in einer materiellen Evidenz zu verankern, die jedoch so aufgestellt ist, dass sie selbst unklar und verstörend bleibt. Im Fall der Forschungsgruppe mit dem Namen Liebesorte in der Schule waren es die Beobachtungen der mitforschenden Mädchen beziehungsweise ihr Pausenprotokoll, das in unseren Gesprächen diese Funktion übernahm. Das Gelingen solcher Verfahren misst sich daran, dass die geteilten Gedanken über sich hinauswachsen, dass die Art des Denkens und die Art, wie gesprochen wird, neue Wendungen nehmen kann, Dinge neu und anders miteinander in Beziehung gesetzt werden. Eine Voraussetzung dabei ist ein guter Balance-Akt zwischen der Strukturierung der Gespräche, also dem Übernehmen von Verantwortung für das Setting, und der wertschätzenden, offenen Haltung allen Teilnehmenden gegenüber. Die gemeinsame Bezugnahme auf das rätselhafte Ding außerhalb - das Kunstwerk, das Beobachtungsprotokoll - erleichtert es dabei, eine momentane Konfiguration der Gleichheit in Bezug auf dieses Ding herzustellen. Diese Momente zeigen sich durch das Entdecken neuer Aspekte, das Staunen über Sichtweisen, über Formen und Strukturen. Dies kann miteinander geteilt werden und so kann, in Bezug auf dieses Dritte, eine gemeinsame forschende Haltung hergestellt werden. Die einzelnen Teilnehmenden zeigen und entwickeln unterschiedliche Wissensbestände diesem Ding gegenüber, diese aber gehen in ihm nie vollständig auf. Es ist diese schwer zu beschreibende Haltung, die sich oft in kleinen Gesten wie Nicken oder Blickkontakt, Nachfragen oder Verweisen zeigt und die eine Forschungsstimmung in eine Gruppensituation bringen kann. Ein wesentliches Element ist weitergehendes Nachfragen und vor allem die Verknüpfung unterschiedlicher Statements, die in der Gruppe geäußert wurden, so wie das Einbringen spontaner Ideen dazu, wie etwas noch von einer anderen Seite angesehen werden könnte.

Ich habe in meinem Beitrag Über die Schwierigkeit nicht rassistisch zu/zuschreiben (Schneider 2017) ein Denken in Widersprüchen beschrieben, die sich in jenen Vermittlungsprozessen formten. Ich versuchte, die Widersprüche meines eigenen Arbeitens und Denkens in der Publikation sichtbar zu machen. Zentral war für mich dabei die Erkenntnis, dass es gerade die 
gelungene Partizipation sein kann, die eine zur Co-Produzentin von Ausschlüssen und rassistischen Zuschreibungen macht. Gleichzeitig kann das Gelingen von Partizipation im Sinne einer engagierten Gestaltung der Prozesse durch alle Beteiligten aber auch Grundlage und Voraussetzung für ein Zurückweisen dieser Ausschlüsse und Zuschreibungen werden. Im Folgenden werde ich versuchen, einige diese Widersprüche zu exemplifizieren und als Folge einer Re-Lektüre einiger Protokollstellen und Analysen zu rereflektieren.

\section{Liebesorte in der Schule}

Beiden oben genannten Sparkling-Science-Projekten war gemeinsam, dass die Schüler*innen die Themen erforschten, die sie interessierten, und zwar unabhängig davon, ob wir als Wissenschaftler*innen oder Vermittler*innen dieses spezifische Interesse unmittelbar teilten. ${ }^{4}$

In der ersten Projektphase von Tricks of the Trade hatten wir die Idee, die Schüler*innen zu bitten, uns die Schule zu zeigen. Zwei Mädchen, Mavi und Susanne ${ }^{5}$, führten mich in "geheime, dunkle« Gänge, in Sackgassen und hinter halb verschlossene Türen, und erzählten mir, dass mensch sich hier treffen könne, ohne den Blicken der Lehrer*innen ausgesetzt zu sein, zumindest potenziell. Schon bald fiel der Begriff Liebesorte und Susanne meinte, dass sie zu Liebe forschen wollen würde. Daraus entstand das spätere Thema der kleinen Forschungsgruppe, bestehend aus Mavi, Susanne und Asly, die noch nicht beim Spaziergang dabei war.

An dieser Stelle sollte ich noch auf etwas verweisen: Es war nicht zufällig, dass wir als Partnerschule eine Wiener Mittelschule und keine gymnasiale Oberstufe wählten, also eine Schule, deren Schüler*innen als bildungsbenachteiligt gelten und das wohl auch sind. Eine solche Schule besuchen in aller Regel Kinder aus nicht akademischen Kontexten, deren Familien oft eine Arbeitsmigrations- oder Fluchtgeschichte haben. In vielen Fällen sind diese Familien jedoch bereits in der dritten Generation in Wien und gelten

\footnotetext{
4 Wöhrer $(2017,166)$ spricht hier von Aushandlungsprozessen.

5 Die Namen sind anonymisiert und folgen den Namen in den von mir dazu veröffentlichten Beiträgen.
} 
dennoch als Ausländer ${ }^{6}$, was auch von den Kindern so beschrieben wurde. Zumindest mich interessierten genau solche Ausgrenzungs- und Ungleichheitsmechanismen entlang dessen, was Paul Mecheril als "Ausländerdispositiv« beschreibt (Mecheril/Rigelski 2007, 61), einem Zusammenspiel von Alltagspraxen, Forschungen, Verordnungen, Geschichten und Diskursen, die bestimmte Menschen als »natio-ethno-kulturell« Andere konstituieren (ebd.). Mich interessiert, wie solche Ausgrenzungen und Zuschreibungen konkret gemacht werden, wie unterschiedliche Menschen an unterschiedlichen Knotenpunkten des Dispositivs daran oft unwillentlich mitwirken und die Strukturen schaffen, in welchen sie agieren. Ebenso interessiert mich, wie sich im Sinne eines Konzepts von Intersektionalität unterschiedliche Diskriminierungspraxen in widersprüchlicher Form miteinander verbinden können, wie also Ausgegrenzte selbst zu Ausgrenzenden werden können. Ich hätte gerne mit den Jugendlichen dazu geforscht, hatte aber keine Idee, wie das gelingen könnte, wenn nur ich das Thema anstoße. $\mathrm{Zu}$ fragen, ob es in der Schule nicht Orte und Nischen gibt, wo etwas passiert, das mit dem Miteinander der Jugendlichen zu tun hat und weniger mit dem Unterricht, und das trotzdem auch Schule ausmacht - diesen Floh hatten mir die Mädchen bei unserem Spaziergang ins Ohr gesetzt. Sie waren offensichtlich wesentlich forscher darin, mich für ihre Frage zu begeistern, als ich mich in der Lage sah, sie für meine zu gewinnen. Das hatte gewiss strukturelle Gründe, wollte ich doch penibel vermeiden, sie zu etwas zu überreden, das vielleicht gar nicht ihr Interesse war. Ich wurde also mit meinen Forschungsanliegen in ihre hineingezogen, dadurch veränderten sich unsere Fragen und es fanden gegenseitige Lernprozesse statt: Die Mädchen diskutierten zusehends über Konfliktlinien und Ausgrenzungspraxen untereinander, ohne dass ich dieses Thema als von mir formulierte Fragestellung auf den Tisch legte, und ich begann meine klare Idee von Ausschluss komplizierter zu denken.

6 Mit der kursiven Schreibweise und der nicht gegenderten Form möchte ich kritisch auf den Diskurs und die politische Praxis verweisen, die Menschen, deren Sprache nicht Deutsch ist und die selbst oder in ihrer Familie Arbeitsmigrations- oder Fluchtgeschichten aufweisen, ausschließlich als Ausländer kategorisieren, unabhängig von ihrem rechtlichen Status oderihrer Selbstwahrnehmung. 


\section{Beobachten beobachten}

Ein Vorhaben dieser Forschungsgruppe war, andere Schüler*innen in der Pause dabei zu beobachten, was sie tun und an welchen Orten sie sich aufhalten. Der Vorschlag kam von mir, die Mädchen fanden ihn gut, änderten jedoch mein Forschungsdesign: Sie beschlossen, nicht am Gang vor ihrer Klasse zu beobachten, sondern in einem anderen Stockwerk. Später erzählten sie mir, dass sie nicht vor Ort Protokoll schrieben, sondern versteckt gemeinsam auf dem Klo. Ich hingegen setzte mich auf die Bank vor ihrer Klasse und folgte dem Treiben davor, was mich wiederum zu einer beobachteten Person machte.

In den anschließend stattfindenden Projekttagen beschäftigten wir uns mit der Frage, was die Beobachtungsposition im Schulkontext bedeutet und wie unterschiedlich ich und die Mädchen diesen Kontext wahrnehmen. Das Protokoll der Mädchen war wesentlich kürzer als meines und beinhaltete kleine Skizzen. Meines glich einem sehr klassischen Beobachtungsprotokoll, in welchem ich versuchte, so genau wie möglich aufzuschreiben, was ich sehen konnte, und persönliche Reflexionen in Klammern hinzuzufügen. Das Protokoll der Mädchen unterschied sich von meinem auch dadurch, dass sie ihr eigenes Wissen und ihre Wahrnehmungen in die Beobachtung einbrachten. Oft protokollierten sie, was sie nicht sehen konnten, sondern vermuteten oder wussten.

An dem Forschungstag und in nachfolgenden Sitzungen malten wir eine ausgedruckte und anonymisierte Version ihres Beobachtungsprotokolls mit unterschiedlichen Farben an: Eine Farbe stand für Ereignisse, die gesehen wurden (»Hans greift Tamara am Arsch an, Tamara schreit lass mich in Ruhe«). Eine andere für Sachverhalte, die vermutet oder unterstellt wurden (»aber trotzdem mag sie das«), die wir sehen, weil wir sie zu wissen glauben oder tatsächlich wissen (»Tamara redet über Ronja, dass sie sie nicht mag!!!!!!«) und so weiter. ${ }^{7}$ Die Diskussion entspann sich daraufhin zunächst nicht entlang der Frage, was sie für Schlüsse aus dem Beobachteten ziehen konnten, sondern darin, was es bedeutete, etwas zu beobachten, etwas zu sehen, wie Sehen und Wissen zusammenhängen: Ich sehe, was ich weiß, manchmal sehe ich auch Dinge, die ich nicht wusste. So diskutierten wir, was es bedeutet,

7 Diese Protokollstellen wurden in unterschiedlichen Beiträgen zitiert, u.a. Wöhrer 2017, 169; dort ist auch das gesamte Protokoll der Mädchen veröffentlicht. 
auf etwas zu schauen; genau hinzuschauen, auch wenn man das eigentlich nicht sollte; eine Meinung über andere zu bilden, weil man hinschaut; und vor allem darüber, was für einen Unterschied es macht, wenn mensch als fremde Person, die schaut, wahrgenommen wird, oder wenn es Freund"innen und Kolleg*innen sind, die das eigene Umfeld beobachten und dieses dadurch als fremd erleben. Auf das von mir aufgehängte Plakat mit der Überschrift »Beobachten« schrieb eines der Mädchen: »Die Person beobachtet ein Opfer und umgekehrt das Opfer beobachtet eine Person « (Wöhrer 2017, 177).

Eine weitere Erkenntnis in diesem Gruppenprozess war, dass das Vertraute einer selbst dadurch fremd erschien, dass es einer Beobachtung unterzogen und das Beobachtete dann in Worte gefasst wurde. Um diesen Prozess von Distanzierung vom Vertrauten durch den Prozess der Analyse zu verdeutlichen, bat ich die Mädchen, aus den Protokollen Worte und Wendungen auszuschneiden, die sowohl mit dem Thema der Forschungsgruppe (Liebesorte in der Schule, Orte, an denen sich Schüler*innen begegnen) als auch dem impliziten Fokus der Beobachtung (Was tun Schüler*innen im Pausengang?) zu tun hatten. So wurde ein Begriffscluster erzeugt und dieser in der Folge noch durch andere Begriffe ergänzt, die in der Diskussion gefallen waren. Meine Idee war, dass sich durch die Entkontextualisierung und Neuanordnung bestimmte Begriffe und damit bestimmte Praxen neu denken lassen.

Die Liste an Begriffen, wie sie von den Mädchen ausgeschnitten und dann geclustert wurden, sah in etwa so aus:

- »betrügen«

• „ «erzählen«

- «über XXX reden, dass jemand sie nicht mag«

- " glauben, dass wer verliebt ist«

- „Wir gehen weg«

- „Sie begrüßen sich«

- „wie Lesben ausschauen«

- „gemein sein«

- »auslachen«

- »kindisch sein, nerven«

- Ich ergänzte beziehungsweise bat sie, dass ich aus der Diskussion noch Begriffe ergänzen darf und fügte diese dem Cluster bei: „Dinge rumerzählen«, »über einander reden«, »wen anschauen«, »verliebt sein - nervös sein«. 
In der gemeinsamen Arbeit mit den Begriffen versuchten wir, diese zu clustern und zueinander neu in Beziehung zu bringen, indem wir sie ausbreiteten und aneinanderlegten. So konnte zum Beispiel »auslachen« mit der Behauptung »wie Lesben ausschauen« oder »über XXX reden, dass jemand sie nicht mag« zusammengebracht werden; ich bat die Mädchen, jeweils Geschichten dazu zu erzählen. Die Neuanordnung der Begriffe konnte auch neue Geschichten zum Vorschein bringen. Dahinter standen Ideen, die nachträglich gesehen mit Hinweisen aus der sozialwissenschaftlichen Methodenliteratur korrespondieren, dass nämlich der bereits durch die Beobachtung und Verschriftlichung einsetzende Prozess der »Verfremdung von Lebenswelten« (vgl. dazu zum Beispiel grundlegend Hirschauer 2010, 207-227; zum Prozess des Protokollierens Merkens 1992) und die Abstrahierung des Gesehenen weiter getrieben wird, um vom konkret Beobachteten zu einem allgemeineren Verständnis sozialer Verhältnisse und Praxen zu kommen.

Nicht nur auf dieser verfremdeten Ebene, sondern beim Analysieren der Protokolle selbst verschoben sich die Fragestellungen von den "Liebesorten in der Schule « hin zu unterschiedlichen Bezugs- und Konfliktfeldern zwischen den einzelnen Jugendlichen und den Blicken, Sprechweisen, Ritualen und Berührungen, die sie prägten.

\section{Widersprüche, in die man selbst verwickelt ist}

Der Forschungstag der kleinen Liebesorte-Gruppe, die Reflexion und die immer neuen Lesarten der Protokolle sowie die Eingriffe in diese, um zu verstehen, wie Sehen und Denken, Beobachten und Entwickeln von Begriffen und Vorstellungen über die eigenen Beziehungsgeflechte zusammenhängen, waren intensiv. Sie machten mich und - so wirkte es auf mich - auch Asly und Mavi glücklich. Das Protokoll und das Plakat, das in dieser Gruppe zum Thema Beobachten entstand, wurde vom wissenschaftlichen Projektteam in unterschiedlichen Zusammenhängen verwendet, um über partizipatives Forschen mit Jugendlichen $\mathrm{zu}$ sprechen und $\mathrm{zu}$ schreiben, sowie auch von mir, um zum Beispiel Vorschläge für unser Praxishandbuch zu entwickeln (vgl. Schneider 2018a; Schneider 2018b).

Susanne, die das Thema Liebesorte ursprünglich vorschlug, war jedoch fast an keinem unserer Treffen und auch nicht bei der Pausenbeobachtung oder der Analyse am Projekttag anwesend. Sie tauchte indirekt in dem Pau- 
senprotokoll der Mädchen auf, als eine Person, über die geredet wird, dass sie nicht gemocht wird. Tatsächlich war dies eine Konstante in unserer Gruppe: Susanne war nicht da, ihr Fortbleiben das Zentrum vieler Gruppentreffen. Mavi versuchte mich immer wieder davon zu überzeugen, dass sie Susanne nicht mag, obwohl sie als innige Freundinnen erschienen, sobald Susanne anwesend war. In diesen Schilderungen, die sich in der Gruppe wiederholten, spielten die Herkünfte der Mädchen eine wesentliche Rolle. So merkte Mavi häufig an, dass sie aus Bosnien und Asly aus der Türkei kommt und Susanne serbische Romni ist.

Es ist für mich im Nachhinein gleichermaßen interessant und verstörend zu sehen, wie ich in jenen Publikationen, die sich nicht explizit mit der Frage des gegen Roma gerichteten Rassismus beschäftigen, diese Frage der Herkünfte auslasse ${ }^{8}$ - auch jetzt im ersten Teil dieses Textes schreibe ich über die gelungenen partizipativen Setzungen und die Konfliktlinien, die sich in und durch diese zeigen, als wären diese Herkünfte und Selbstpositionierungen der Schüler*innen nicht deren eigene, von sich selbst aufgebrachte Fragestellung. Eine solche Haltung ist gewiss davon informiert, dass ich mich bemühte, die Mädchen nicht auf eine herkunftsbezogene Position festzuschreiben. Auch im Gruppenprozess versuchte ich, die Fixierung der Mädchen auf ihre jeweiligen Herkünfte zu vermeiden und damit gewisse Aussagen zu überhören. So stellte ich Mavi die Frage, warum sie behauptet, Susanne nicht zu mögen, obwohl sie doch eindeutig als Freundinnen auftreten. Die folgende Protokollstelle enthält Mavis Antwort:

"Sie, Mavi, hätte sich am Anfang echt bemüht, weil sie ja auch nicht gewusst hätte ob sie, als Bosnierin, mit einer Serbin sprechen könne, aber dann sei sie draufgekommen, dass diese ganz nett sei, aber das wäre eben doch nicht so. Immer wieder geht es auch darum, dass [...] andere Kinder, vor allem der Max sie >Dreckige Zigeunerin « nennen; es ist in den Schilderungen von Mavi nicht so klar, ob sie mehr Probleme mit Susanne hat, die sich das gefallen lässt, und weiter auf Max steht, oder ob sie mehr Probleme mit Max hat, der so etwas sagt. Jedenfalls richten sich alle Aggressionen und Abgrenzungen gegen Susanne.« (Gedächtnisprotokoll Karin Schneider, 9.4.2009)

8 Vgl. z. B. Schneider 2018a, 2018b. Bei Wöhrer $(2017,177)$ ist auf dem Bild des Plakates auch zu lesen »BOSNA Türkei« und darunter ist ein Herz gemalt; in unseren Analysen greifen wir das meist nicht näher auf. 
Ich versuchte in meinem Text Die Schwierigkeit nicht rassistisch zu/zuschreiben (2017) die Ausgrenzungen eines von Rassismus betroffenen Mädchens in der Schule als ein Zusammenspiel unterschiedlicher Akteur"innen und institutioneller Normen zu beschreiben: Ein Zusammenspiel von vermeintlich neutralen Schullogiken und Familienideologien, expliziten Rassismen seitens der Klassenkolleg*innen und dem von eigenen Projektionen beziehungsweise Ignoranz geprägten Agieren seitens der für das jeweilige Setting verantwortlichen Erwachsenen. Ihre Freundinnen scheinen in dieser Anordnung konfus, ratlos und zerrissen. In der Protokollstelle lässt sich sehen, dass ich nicht auf den explizit und drastisch geäußerten Rassismus gegen Susanne eingehe. In der Art, wie ich das Protokoll schreibe, interessiere ich mich auf abstrakter Ebene dafür, was Mavi eigentlich damit sagen will, frage sie das aber nicht.

Die Diskussionen darüber, dass sie Susanne nicht mögen und dass dies ein unter uns dreien geteiltes Geheimnis bleiben sollte, prägten die Treffen vor und nach der Pausenbeobachtung. Dieses für mich faszinierende wie unangenehme »Bündnis«bildete (bewusst und meist unbewusst) die Unterlage der Ansätze der Gruppenmoderation, die ich entwickelte. Mein Stil, geduldig zuzuhören, genau verstehen zu wollen, die Mädchen durch Visualisierungen von Gedanken und Auseinandersetzung mit einzelnen Begriffen stärker in einen gemeinsamen Reflexionsprozess zu verwickeln, erzeugte bei ihnen vielleicht die Stimmung, mir mehr zu erzählen und freier über ihre Verstrickungen zu sprechen. Diese produktiven Effekte meiner spezifischen, sehr interessierten, nicht wertenden und ermutigenden Form der Gesprächsführung überzeugten mich auf der anderen Seite, diese Haltung beizubehalten. Wir drei identifizierten uns offensichtlich mit einem Prozess, an dem eben nur mehr drei und nicht vier teilnahmen. Bei dem die Vierte als störender Faktor herausgehoben, mit Zuschreibungen bedacht und zum Gesprächsthema wurde. Durch meine methodischen Verfahren kam viel auf den Tisch. Die Mädchen redeten offen über Rassismus, zeigten ihn vor und reproduzierten ihn. Was ich mit meinen Methoden und Haltungen nicht vermochte, war ein Setting zu schaffen, in welches Susanne potenziell zurückkommen konnte. Ich hörte zu, stellte Fragen, vermied Wertungen und versuchte, mit meinem zunehmenden Unwohlsein nach den Situationen durch das Schreiben der Protokolle reflektierend umzugehen. So konnte ich Mavis ständiges Insistieren, mir über den gegen Susanne gerichteten Rassismus zu erzählen und ihn zu performen, nicht als Angebot sehen, in der Gruppe tatsächlich 
darüber zu sprechen. Zugespitzt könnte man sagen, dass die Mädchen, vor allem Mavi, längst Rassismus in ihrem eigenen Umfeld und die für sie damit verbundenen persönlichen, inneren Konflikte zum Thema der Forschungsgruppe machten, während ich noch damit beschäftigt war, durch Umschiffen und Vermeiden einer expliziten Positionierung das Einverständnis in der Gruppe nicht zu gefährden. Je stärker ich versuchte, Methoden einzuführen, die halfen, Gesagtes zu abstrahieren und auf eine andere Ebene zu bringen, umso mehr lenkte ich (unbewusst, aber manchmal sehr bewusst) von dem eigentlichen Thema dieser Gruppe (»Was Rassismus mit meinen eigenen Freundschaften tut«) ab. Je mehr ich dies tat, umso mehr brachte ich Mavi in die Situation die durch diese Methoden geschaffenen Freiräume dafür zu nutzen, auf diesem Thema zu insistieren und es immer und immer wieder einzubringen.

Dennoch schufen meine methodischen Ansätze und die Art, wie ich moderierte, für diese Gruppe produktive Voraussetzungen, mit diesem Thema umzugehen. Im nächsten Abschnitt werde ich dies genauer beschreiben.

\section{"Die Welt, wie ich sie gerne hätte "}

Susanne war bei einem unserer Nachbesprechungstreffen wieder in der Schule. Mavi erinnerte mich an mein Versprechen, dass alles, was in der Gruppe gesagt wurde, unter »uns« bleiben und Susanne nichts davon erfahren solle. Susanne ihrerseits war nicht nur anwesend, sondern forderte ganz deutlich ihr Recht ein, Teil der Gruppe zu sein und erschütterte damit die Geschichten, die ich mir über die Richtigkeit meiner Zurückhaltung bis dahin erzählte.

Ich bat die drei in dieser Situation, gemeinsam im Protokoll jene Stellen zu markieren, die eine Auskunft darüber geben, wer wen auslachen würde und warum. Mavi und Asly hatten zu diesem Zeitpunkt bereits eine Erfahrung in dieser Art des Clusterns und markierten im Protokoll jene Menschen, die mit Ausgelachtwerden in Zusammenhang gebracht wurden: Lesben, Integrationskinder, Einzelne, die im Protokoll namentlich genannt wurden. Sie gestalteten dann auf meine Anregung hin eine Tabelle: Wer wird ausgelacht? Von wem? Wann? Wo? Mit welcher Konsequenz? ${ }^{9}$ Danach fragte ich

9 Diese Vorgehensweise wurde von mir in der Methodenanalyse überarbeitet und beschrieben in Schneider 2018a, 105. 
die Mädchen, ob sich Auslachen an bestimmte Gruppen bindet. Die folgende Protokollstelle gibt die darauf einsetzende Diskussion wieder:

»Mavi erklärt mir, dass die Integrationskinder immer ausgelacht werden, dass die sozusagen ganz unten stehen, die andere stimmen zu. Dann sagt sie, auch die Kopftuchkinder werden ausgelacht. Ich sprechen Asly (die Kopftuchträgerin ist) direkt drauf an, sie sagt ja, aber dass ihr das ziemlich wurscht ist. Sie erzählt eine Begebenheit aus dem Turnsaal, wo Buben gesehen haben, dass ihr das Kopftuch verrutscht ist und sich total drüber lustig gemacht haben. Aber sie dann auch in die Bubengarderobe geschaut hat und auch drüber (gemeint ist über Dinge, die sie bei den Buben gesehen hat) gelacht hat; und sie erzählt, dass die anderen (Buben?) von der Kopftuchmafia reden und ich frage sie und die anderen beiden, ob es denn so was überhaupt gäbe, große Empörung und Kopfschütteln bei allen. So, sage ich, da wird also wegen was ausgelacht, wo du Mitglied bist, das es gar nicht gibt. Großes Gelächter! Und, frage ich, wie ist das unter euch, seid ihr eher auf der Seite der Auslachenden oder der Ausgelachten? Also ich, sagt Susanne, habe auch schon mal ausgelacht, nämlich den Berni, und das ist eigentlich gemein, da hab' ich mich so drangehängt an alle; alle sind sich einig, dass Berni irgendwie komisch ist, und auch einer von den Buben, die das Etikett schwul bekommen ohne es zu sein. Also meine Mama, sagt Susanne, die sagt immer, besser ein schwuler Mann als ein elender anderer, die schwulen Männer sind die besten Freunde.« (Cedächtnisprotokoll Karin Schneider, 15.5.2009)

An dieser Protokollstelle zeigt sich, dass die Hierarchien zwischen Auslachenden und Ausgelachten manchmal zwar eindeutig sind - so stehen die Integrationskinder ganz unten. Danach kommen die Mädchen, die Kopftuch tragen (und von Buben ausgelacht werden, die in dieser Schule wesentlich aus islamischen Kontexten kommen), dann die »schwulen« Buben und so weiter. Jedoch verorteten sich die einzelnen Mädchen der Forschungsgruppe jeweils unterschiedlich entlang der Hierarchien und sie nahmen sich die Freiheit heraus, nicht nur nicht in einem Opferstatus (als kopftuchtragende Muslima, als Migrantin, als Romni, als Mädchen) zu bleiben, sondern »zurück auszulachen« und sogar, im Falle Susannes, die eigene auslachende Haltung zu hinterfragen.

Der nächste Schritt in dieser Gruppendiskussion war, dass ich fragte, ob es noch eine Gruppe gäbe, die zur Zielscheibe von gemeinen Bemerkungen 
würde. An dieser Stelle fragte Mavi Susanne etwas auf Bosnisch/Kroatisch/ Serbisch und übersetzte dann, dass sie gefragt habe, ob sie erzählen dürfe, dass die Kinder »dreckige Zigeunerin« sagten, und Susanne hätte darauf gemeint, dass ihr das egal sei. Susanne erzählte, dass sie ihre vorherige Schule verlassen musste, weil sie sich dort mit ihren Roma-Freundinnen gegen den Rassismus von türkischen ${ }^{10}$ Mitschüler"innen tatkräftig gewehrt hätte. Mavi und Asly hörten Susannes Schilderung zu, stellten Fragen, äußerten Empörung. ${ }^{11}$ Ich schlug unmittelbar nach dieser Diskussion vor, Plakate zu machen mit der Überschrift:»Die Welt, wie ich sie gerne hätte«. Mavi bestand darauf, dass sie und Susanne das Plakat gemeinsam machen. Für Asly war dies insofern in Ordnung, als dass sie ohnehin ein eigenes Plakat machen wollte und zwar zum Thema »Die Welt ohne Buben«. An dieser Stelle endet die Geschichte der Forschungsgruppe Liebesorte in der Schule. Sie endet sehr abrupt, weil dies aufgrund der Rahmenbedingungen des Projekts tatsächlich das letzte Gruppentreffen war. Die Plakate waren Teil der eher beiläufigen, weil zeitknappen Schlusspräsentation und wurden nicht speziell gewürdigt, wurden von mir nicht dokumentiert und fanden auch nicht Eingang in weitere Artikel und Besprechungen. $\mathrm{Zu}$ diesem Zeitpunkt war diese Projektphase zu Ende, die Abschlusspräsentation nur eine kleine Angelegenheit kurz vor der Pausenglocke. Nichts wies in der Schlusspräsentation dieser kleinen Forschungsgruppe darauf hin, was es politisch und persönlich bedeutete, dass es diese Plakate gab und dass Mavi und Susanne ihres zusammen gestalteten. Diese Plakate existieren einzig in meiner Erinnerung daran, dass es hier eigentlich hätte beginnen müssen, dass wir erst zu diesem Zeitpunkt jene Forschungsgruppe wurden, die wir vorher nur vorgaben zu sein. Eine Gruppe, in der eigene Praxen und Erlebnisse des Ausschließens und Ausgeschlossenwerdens befragt werden und daraus eine Utopie entwickeln kann. Während die Mädchen über Liebe und ich über Ausschlüsse forschen wollten, haben wir gemeinsam gelernt, wie beides zusammenhängt.

\footnotetext{
10 Ich hielt es ursprünglich für problematisch hier national auszuweisen. Da aber genau diese Zuschreibungen und Identitätskonstruktionen in den Erzählungen und Bezugnahmen der Mädchen eine große Rolle spielten, entschloss ich mich letztendlich dafür.

11 Für eine genaue Schilderung und Analyse dieser Situation siehe Schneider 2017.
} 


\section{Literatur}

Hirschauer, Stefan (2010). Die Exotisierung des Eigenen. Kultursoziologie in ethnografischer Einstellung, in: Wohlrab-Sahr, Monika (Hg.): Kultursoziologie Paradigmen - Methoden - Fragestellungen, Wiesbaden: VS Verlag für Sozialwissenschaften, 207-227. https://doi.org/10.1007/978-3531-92300-0_9.

Hubin, Andrea/Schneider, Karin (2017). Dritte Sachen und Denkbilder. Methodische Überlegungen zur Neuanordnung von Redeweisen in Kunstvermittlungsaktionen, in: Kettel, Joachim (Hg.): Tagungsband zur Konferenz The Missing_LINK 2016. Übergangsformen von Kunst und Pädagogik in der kulturellen Bildung, Oberhausen: Athena, 453-462.

Mecheril, Paul/Rigelsky, Bernhard (2007). Nationaler Notstand. Ausländerpädagogik und Ausländerdispositiv, in: Riegel, Christine/Geisen, Thomas (Hg.): Jugend, Zugehörigkeit und Migration. Subjektpositionierung im Kontext von Jugendkultur, Ethnizität und Geschlechterkonstruktionen. Wiesbaden: VS Verlag für Sozialwissenschaften, 61-80. https://doi. org/10.1007/978-3-531-90480-1_3.

Merkens, Hans (1992). Teilnehmende Beobachtung: Analyse von Protokollen teilnehmender Beobachter, in: Hoffmeyer-Zlotnik, Jürgen H. P. (Hg.): Analyse verbaler Daten: über den Umgang mit qualitativen Daten, Opladen: Westdeutscher Verlag, 216-247. https://doi.org/10.1007/978-3-32290092-0_8.

Schneider, Karin (2017). Über die Schwierigkeit nicht rassistisch zu/zuschreiben, in: Wöhrer, Veronika/Arztmann, Doris/Wintersteller, Teresa/Harrasser, Doris/Schneider, Karin: Partizipative Aktionsforschung mit Kindern und Jugendlichen. Von Schulsprachen, Liebesorten und anderen Forschungsdingen. Wiesbaden: Springer VS, 239-262. https://doi.org/10.1007/978-3-65 8-13781-6_16.

Schneider, Karin (2018a). Auswertung von Beobachtungsprotokollen, in: Wöhrer, Veronika/Arztmann, Doris/Wintersteller, Teresa/Harrasser, Doris/Schneider, Karin: Praxishandbuch Sozialwissenschaftliches Forschen mit Kindern und Jugendlichen. Weinheim/Basel: Beltz Juventa, 102-108.

Schneider, Karin (2018b). Teilnehmende Beobachtung, in: Wöhrer, Veronika/ Arztmann, Doris/Wintersteller, Teresa/Harrasser, Doris/Schneider, Karin: Praxishandbuch Sozialwissenschaftliches Forschen mit Kindern und Jugendlichen. Weinheim/Basel: Beltz Juventa, 78-83. 
Sturm, Eva (2012). Die Position »von Kunst aus« in 9 Punkten dargelegt. Rede für kunstvermittlungs-interessierte Leserinnen und Leser (Text mit Klammern). Oder: Vom Arbeiten mit Kunst. Onlinepublikation des Modellprogramms Kulturagenten für kreative Schulen 2011-2015, Berlin, abrufbar unter: http://publikation.kulturagenten-programm.de/detailansicht. html?document=161\&page=reflexion.html (letzter Zugriff: 8.8.2019).

Wöhrer, Veronika/Arztmann, Doris/Wintersteller, Teresa/Harrasser, Doris/ Schneider, Karin (2017). Partizipative Aktionsforschung mit Kindern und Jugendlichen. Von Schulsprachen, Liebesorten und anderen Forschungsdingen. Wiesbaden: Springer VS. https://doi.org/10.1007/978-3-658-13781-6. Wöhrer, Veronika (2017). (Wie) kommen wir vom >I and $\mathrm{I}$ zum >Weく? Wer sind die Forschungssubjekte von PAR mit Kindern und Jugendlichen?, in: Wöhrer, Veronika/Arztmann, Doris/Wintersteller, Teresa/Harrasser, Doris/Schneider, Karin: Partizipative Aktionsforschung mit Kindern und Jugendlichen. Von Schulsprachen, Liebesorten und anderen Forschungsdingen. Wiesbaden: Springer VS, 165-176. https://doi.org/10.1007/978-3658-13781-6_9.

Wöhrer, Veronika/Arztmann, Doris/Wintersteller, Teresa/Harrasser, Doris/ Schneider, Karin (2018). Praxishandbuch Sozialwissenschaftliches Forschen mit Kindern und Jugendlichen. Weinheim/Basel: Beltz Juventa. 



\title{
Making Art, Taking Part! \\ DIY-Kulturen und künstlerische Interventionen im Kontext einer kritischen Vermittlungspraxis
}

Elke Zobl

\begin{abstract}
Wie können kulturelle Do-it-yourself-Praktiken und künstlerische Interventionen Jugendlichen Handlungsräume und Möglichkeiten der gesellschaftlichen Mitgestaltung eröffnen? Welche Blickwinkel, aber auch Reibungen und Ambivalenzen ergeben sich, wenn wir gemeinsam mit Jugendlichen forschen? Die Verknüpfung einer Perspektive, die herrschende soziale und kulturelle Ordnungen hinterfragt, mit Konzepten, die zu eigenen kulturellen Produktionen anregen, bietet eine gute Möglichkeit, Handlungsräume zu eröffnen, und stellt zugleich eine Herausforderung für die schulische und außerschulische Arbeit dar. Im Zentrum dieses Beitrags stehen zwei Vermittlungsprojekte mit Jugendlichen an der Schnittstelle von Universität, Schule und Gesellschaft. ${ }^{1}$
\end{abstract}

\section{Ausgangspunkte}

Ich stelle im Folgenden die am Programmbereich Zeitgenössische Kunst und Kulturproduktion (Schwerpunkt Wissenschaft \& Kunst, eine Kooperation von Universität Salzburg und Universität Mozarteum Salzburg) durchgeführten Projekte Making Art, Making Media, Making Change! und Making

1 Dieser Text baut auf verschiedenen Beiträgen mit dem Team des Programmbereichs und der Projekte auf (vgl. u. a. Aqra/Huber/Smodics 2016, Zobl 2016, Drüeke/Zobl 2012, Klaus/ Zobl 2019, Zobl/Reitsamer/Crünangerl 2012, Zobl/Huber 2016, Zobl/Klaus/Moser/Baumgartinger 2019). Ich danke allen herzlich für die Zusammenarbeit! 
Art-Taking Part! vor, um Möglichkeiten der Verbindung einer gesellschaftskritischen Perspektive mit eigener kultureller Produktion aufzuzeigen und zur Diskussion zu stellen. Grundlegend für die Herangehensweise an diese Projekte sind drei konzeptionelle Ausgangspunkte:

\section{Kritische Kulturproduktion und der Kulturbegriff der Cultural Studies}

Im Sinne der Cultural Studies ist Kultur eine dynamische und konflikthafte Praxis, die im Alltagshandeln verschiedener Gruppen und Klassen ihren Ausdruck findet. Das traditionelle westliche, bürgerliche Verständnis von Kultur ist geprägt durch Abgrenzung und Hierarchisierung zwischen gesellschaftlichen Schichten und zwischen vermeintlich Eigenem und Fremdem (vgl. Mörsch 2016, o. S.). In solch ein elitäres, von der Idee einer Hochkultur getragenes Verständnis intervenieren die Cultural Studies. ${ }^{3}$ Sie forcieren eine Perspektive, die künstlerische Ausdrucksformen und alltagskulturelle, mediale Produktionen auf eine Ebene stellt. Dabei rücken sie die Analyse von Macht und Ungleichheiten, von Ausschlüssen, aber auch von Selbstrepräsentation und Ermächtigung, Solidarität und gesellschaftlicher Verantwortung in den Fokus. Hier spielt die Erkenntnis eine grundlegende Rolle, dass Menschen in die kulturelle Bedeutungsproduktion und die damit verbundenen Machverhältnisse eingreifen können, indem sie selbst zu aktiven Kulturproduzent"innen werden (vgl. Zobl et al. 2019). Dieser Kulturbegriff ist eingebettet in das übergreifende politische Ziel, Gesellschaftsveränderung im Sinne von Demokratie, Inklusion und Emanzipation aller Menschen $\mathrm{zu}$ erreichen.

2 Making Art, Making Media, Making Change! (https://www.makingart.at) wurde im Rahmen eines FWF-Wissenschaftskommunikationsprojektes 2014-2015 und Making Art - Taking Part! (https://www.takingpart.at) im Rahmen des Sparkling-Science-Programms 2014-2016 durchgeführt. Die dabei entstandenen Vermittlungsformate und Materialien werden laufend weiterentwickelt.

3 Mörsch weist darauf hin, dass verschiedene Bewegungen an der Erweiterung des Kulturbegriffs beteiligt waren, wie »die europäischen Bewegungen der Arbeiterbildung, [...], die sich gegen die smusische Bildung abgrenzende skulturelle Bildung in der BRD, die lateinamerikanische Befreiungspädagogik oder die Widerstandsbewegungen der Dekolonisierung und der Indigenen« (Mörsch 2016, 0. S.). 


\section{Kritische Kulturvermittlung}

Unser Interesse gilt der Entwicklung einer Kulturvermittlung als kritische Praxis unter Bezugnahme auf Ansätze, die Bildung als Werkzeug zur Hinterfragung von Machtverhältnissen und zur Selbstermächtigung verstehen (vgl. hooks 1994; Freire 1978 [1970]). Zentral dabei ist das Öffnen von Räumen, in denen kulturelle Teilhabe- und Handlungsmöglichkeiten für möglichst viele gesellschaftliche Gruppen entstehen können. Aus unserer Perspektive ist es wichtig, vielfältige Formate, Vermittlungsmaterialien und Angebote $\mathrm{zu}$ entwickeln, die Möglichkeiten der Selbstrepräsentation und gesellschaftskritische Momente aus einer antirassistischen und queer-feministischen Perspektive mitdenken beziehungsweise als Ausgangspunkt nehmen. ${ }^{4}$

\section{Verbindung von Forschung und Praxis: Partizipativ und transdisziplinär forschen}

Unsere Arbeitsweise basiert auf der starken Verbindung von Forschung und Praxis. In den Vermittlungsprojekten arbeiten wir mit Ansätzen der partizipativen Forschung, die den Anspruch des Eingreifens in gesellschaftliche Kontexte und deren Veränderung durch das gemeinsame Forschen verfolgen. Dabei können neue Formen der Wissensproduktion entstehen (vgl. von Unger 2014, 6). Grundlegend dafür sind inter- und transdiziplinäre Teams, um unter Beteiligung von Gruppen und Individuen, die außerhalb der Wissenschaft stehen, gemeinsam und vielstimmig zu arbeiten und zu forschen. Hier bildet Arbeit mit Kindern und Jugendlichen im Rahmen einer Aktionsforschung eine wesentliche Möglichkeit (vgl. Wöhrer et al. 2017).

4 Für eine Zusammenstellung solcher Materialien siehe das Archiv für emanzipatorische Praxen, das im Projekt Strategien für Zwischenräume. Neue Formate des Ver_Lernens in der Migrationsgesellschaft von trafo.K entwickelt wurde (http://www.verlernen.trafo-k.at/index. php), und die Materialiensammlung auf der Website des Projektes Taking Part! (https:// www.takingpart.at, letzter Zugriff: 29.9.2019). 


\section{Projektbeispiele}

\section{Making Art, Making Media, Making Change! DIY-Kulturen als Perspektive}

Welche Bilder werden im Alltag und in den Medien verwendet, um Geschlechterrollen herzustellen? Wie werden Ungleichheiten und Heteronormativität reproduziert? Warum besteht die Notwendigkeit, alternative Medien selbst zu produzieren? Wie kann eine Praxis des Selbermachens zum Ausdruck von Kritik werden? Inwiefern kann das, was von der Gesellschaft als 'normak und sschön angesehen wird, hinterfragt und umgedeutet werden - welche Gegenbilder können dem entgegengesetzt werden? Und produzieren diese ihrerseits wiederum Normen?

Solche und ähnliche Fragen standen im Zentrum des Wissenschaftskommunikationsprojektes Making Art, Making Media, Making Change! (20142015). Ziel war es, Jugendlichen Einblicke in gegenwärtige alternative Medien- und Kulturproduktionen mit feministischem und antirassistischem Anspruch zu geben, ihnen Handlungsräume als aktive kulturelle und mediale Produzent"innen zu eröffnen und Prozesse des Fragenstellens in Richtung eines machtkritischen Denkens auszulösen.

Ausgangspunkte waren verschiedene kulturelle Praktiken von Jugendlichen: Zines (selbstgemachte und -verbreitete Magazine) ${ }^{5}$, Comics und Critical Crafting (Verbindung von Handarbeit und Aktivismus, auch als Craftivism bezeichnet; vgl. Müller 2007; Reitsamer/Zobl 2011; Zobl 2009; Zobl 2011) stehen in einer engen Verbindung zu Do-it-yourself-Kulturen. Diese charakterisieren sich durch Selbstorganisation, durch das Aufbrechen der Grenzen zwischen Konsumierenden und Produzierenden und durch nichtformalisierte Lernpraktiken. DIY-Akteur*innen versuchen, ihre kulturellen Produktionen möglichst unabhängig von kommerziellen Strukturen und einer kapitalistischen Waren- und Kulturindustrie herzustellen und zu verbreiten (vgl. Langreiter/Löffler 2017; Reitsamer 2013). Kulturelle Praktiken

5 Nachdem ich vor zwanzig Jahren feststellen konnte, dass einerseits die Forschung sich auf Zines im anglo-amerikanischen Raum fokussierte und andererseits eine zentrale, virtuelle Ressourcenseite fehlte, gründete ich 2001 das Online-Archiv Crrrl Zine Network (http:// www.grrrlzines.net, letzter Zugriff: 29.9.2019), um aufzeigen, dass Zines in vielen verschiedenen Ländern (in meiner Studie in 43 verschiedenen Ländern) produziert werden. Das Folgeprojekt Grassroots Feminism (http://www.grassrootsfeminism.net) hat einen breiteren Fokus auf verschiedene DIY-Praktiken. 
und alternative Medien spielen seit jeher eine wichtige Rolle in sozialen Bewegungen. Sie bieten die Möglichkeit einer demokratischen Kommunikation, der Artikulation von heterogenen Sichtweisen, der Vernetzung, der Selbstermächtigung und der Teilhabe.

Auf dieser inhaltlichen Basis haben wir mit den Künstlerinnen $\mathrm{Ka}$ Schmitz und Stephanie Müller sowie weiteren Kooperationspartner*innen ${ }^{6}$ verschiedene Workshops zu alternativer Medien- und Kulturproduktion entwickelt und durchgeführt: eine Experimentierwerkstatt, einen Comicund einen Zine-Workshop.

Über den Einstieg in die Workshops durch die Primärmaterialien der Zines und Comics ist es möglich, direkt vielfältige Themen anzusprechen und so einen Handlungsraum zu öffnen, der von den Teilnehmer*innen mitbestimmt wird, indem sie ihre Meinungen und Lebenswirklichkeiten einbringen und diese - teilweise kritisch - in Bezug zu gesellschaftlichen Verhältnissen setzen (vgl. Zobl/Drüeke 2016). In diesem Raum können grundsätzliche Denk- und Reflexionsprozesse initiiert werden, um gesellschaftliche Machtverhältnisse und Ausschlüsse zu hinterfragen. ${ }^{7}$ Bei den Bildkarten und dem Collagieren für die Zines greifen die Workshopleiter*innen vorwiegend auf Zeitungen aus feministischen und frauenpolitischen Kontexten zurück, um den Mehrheitsdiskurs nicht von vornherein zu reproduzieren.

\section{Making Art, Taking Part! Künstlerisches und kulturelles Intervenieren mit Jugendlichen}

Wie kann ein Eingreifen in Öffentlichkeiten mit künstlerischen und kulturellen Strategien von und mit Jugendlichen erprobt und reflektiert werden?

6 Durchgeführt wurde das Projekt mit Kooperationspartner*innen der schulischen und außerschulischen Jugend- und Mädchenarbeit (Teresa Lugstein von make it - Büro für Mädchenförderung des Landes Salzburg, MonA-Net: Mädchen Online Netzwerk Austria, Frauenbüro Stadt Salzburg), Carmen Mörsch (Institute for Art Education, Zürcher Hochschule der Künste), Büro trafo.K, Ka Schmitz, Stephanie Müller, Red Chidgey, ger2, Kulturund Medienproduzent*innen, dem Grrrl Zine Archiv am gendup - Zentrum für Gender Studies und Frauenförderung der Universität Salzburg sowie Studierenden der Universität Salzburg und dem Mozarteum Salzburg. Dem Team gehörten Ricarda Drüeke, Stefanie Grünangerl und Elke Zobl an.

7 Vor allem Jugendliche, die sich marginalisiert fühlen, sich im Prozess des Outings befinden oder Diskriminierungserfahrungen gemacht haben, empfinden es als positiv, dass diese Erfahrungen zur Sprache gebracht werden. 
Öffentlichkeit ist ein Prozess, in dem gesellschaftliche Übereinkünfte erzielt werden (vgl. Klaus 2006). In dem Projekt Making Art - Taking Part! (2014-2016) entwickelten die 14- bis 16-jährigen Schüler*innen zweier Klassen in Stadt und Land Salzburg8 gemeinsam mit Künstler"innen und dem Projektteam Interventionen im öffentlichen Raum. Der Vermittlungsfokus lag darauf, Fragen und Anliegen der Schüler*innen aufzugreifen und dazu gemeinsam Formate der Intervention und Möglichkeiten der Herstellung partizipativer Öffentlichkeiten zu erproben.

Bei der Zusammenarbeit mit den Schüler*innen der NMS Liefering, einer am Rand der Stadt Salzburg gelegenen Schule mit sehr vielen Schüler*innen mit Erfahrungen von Ausschlüssen und Migration, stellte sich als durchgängige Frage »Wie zusammenleben?« heraus. In der Folge wurden zwei Schwerpunkte bearbeitet: zum einen die »Produktion von Ungleichheiten« und zum anderen »Zukunftsvisionen einer anderen Gesellschaft«.

Um die Inhalte für die Intervention $\mathrm{zu}$ entwickeln, diskutierten wir in Kleingruppen die Begriffe Protest, Sprache, Stereotyp, Rassismus und Antirassismus (zu den verwendeten Definitionen siehe trafo.K 2011). Im Gespräch über die Begriffe wurden Zukunftsvisionen entwickelt, wie ein gelungenes Zusammenleben ausschauen könnte: Welche Handlungsstrategien können wir gegen Ausgrenzung finden? Auf der Ebene der Vermittlungstools regten wir als Team, aufbauend auf den Ideen der Schüler*innen, an, einen mobilen Infowagen zu bauen, diesen als Tauschbörse (von Ideen, Materialien etc.) zu begreifen und Aktionen mit den Passant"innen zu initiieren, um diese in einen inhaltlichen Austausch einzubeziehen. Für eine Würfelinstallation malten die Schüler*innen zentrale Wörter für das Zusammenleben auf die Würfel - wie »verstehen«, "anerkennen«, »lieben«, »offen sein", »mitbestimmen« oder »respektieren « - und übersetzten diese in die in der Klasse gesprochenen Sprachen. Zudem entwickelten sie gemeinsam mit der Künstlerin Moira Zoitl ein

8 In diesem Sparkling-Science-Projekt arbeiteten wir mit einer 4. Klasse der NMS Liefering in der Stadt Salzburg und einer 5. Klasse im BORC Mittersill im Pinzgau im Bundesland Salzburg zusammen. Das Team umfasste Veronika Aqra, Laila Huber, Elke Smodics und Elke Zobl. Das Projekt wurde in Kooperation mit den Künstler_innen Marty Huber, Moira Zoitl, Katharina Kapsamer, Stephanie Müller und Klaus Dietl sowie mit Elisabeth Klaus und Ricarda Drüeke, Fachbereich Kommunikationswissenschaft, Universität Salzburg, Iwan Pasuchin, Pädagogische Hochschule Salzburg, Carmen Mörsch, Institute for Art Education, Zürcher Hochschule der Künste, Hans Holzinger und Walter Spielmann, Robert-JungkBibliothek für Zukunftsfragen Salzburg, und Büro trafo.K durchgeführt. 
fotografisches Gesten-ABC zu zentralen Begriffen wie Gleichberechtigung, Solidarität, Freundschaft oder Protest. Beim Interaktionsangebot "Slogans und Fragen zum Pflücken« konnten auf Kärtchen gedruckte Slogans und Fragen nach Zufallsprinzip kombiniert und als Startpunkt für Gespräche zum Thema "Wie zusammenleben?« genutzt werden (Zobl/Huber 2016). Die Ideen dazu wurden von Workshop zu Workshop mit den Schüler*innen gesammelt, aber die Fokussierung und Bündelung der Formate erfolgte durch das Projektteam.

Im Laufe des Prozesses stellte sich heraus, dass wir zu wenig Zeit für eine Reflexion über den Forschungsprozess mit den Schüler*innen eingeplant hatten. Um die Projekterfahrung zu reflektieren, arbeiteten zwei Projektmitarbeiterinnen aufgrund einer Anregung in einem Roundtable ${ }^{9}$ im Anschluss an die Zusammenarbeit mit der Klasse mit vier (ehemaligen) Schüler*innen in Form eines bezahlten Praktikums zusammen. Die Praktikant*innen wählten für die Prozessreflexion das Publikationsformat einer Digital Story, die wir mit dem Titel »Kunst hat viele Ansichten $\aleph^{10}$ veröffentlichten.

\section{Reibungen und Widersprüche zwischen Anspruch und Handlungsmöglichkeiten}

In den Projekten haben sich zahlreiche Fragen, Widersprüche und Reibungen ergeben, die ich in Rückbezug auf kritische Kulturproduktion und Kulturvermittlung sowie die Verbindung von Theorie und Praxis reflektieren möchte.

\section{Kultur als Praxis und traditioneller Kunstbegriff}

Wenn wir von den Alltagserfahrungen und kulturellen Praktiken der Menschen ausgehen, dann hat dies tiefgreifende Konsequenzen für die Kulturvermittlung und die edukative Praxis. So fragt der kanadische Erziehungswissenschaftler Rubén Gaztambide-Fernández (2014) danach, was das Etikett 'Kunst und welche Konsequenzen die inhaltliche Rahmung mit `Kunst in

\footnotetext{
9 Ich danke Carmen Mörsch für ihr Feedback und ihre Anregungen im Rahmen eines Roundtables zu Kritischer Kunstvermittlung am 23. September 2015 in Salzburg.

10 Die Digital Story wurde in der Abschlussausstellung gezeigt sowie auf der Projektwebsite und in der Ausgabe Nr. 7 des ejournals p-art-icipate - Kultur aktiv gestalten des Programmbereichs publiziert (https://www.p-art-icipate.net/cms/kunst-hat-viele-ansichten/)
} 
der Schule bedeuten. Der Kunstbegriff sei mit einer Wirkungsrhetorik und einem eurozentrischen, elitären Verständnis verknüpft. Ausschlüsse würden im Sinne sozialer Distinktion (re-)produziert. Er plädiert daher für ein Verständnis von Kultur als Praxis und dafür, den Fokus auf das Tun der beteiligten Akteur*innen zu richten. Dieser Vorschlag, den Kunstbegriff in der Schule zugunsten eines Verständnisses von »symbolischer und kultureller Arbeit« beziehungsweise »symbolischer Kreativität« (Gaztambide-Fernández 2014, 56) als kulturelle Produktion außen vor zu lassen, kann eine Verschiebung des Blickes bedeuten und einen Handlungsraum für die Schüler*innen in Bezug auf ihre eigenen kulturellen Praktiken eröffnen.

Genau dies konnten wir in den beiden skizzierten Projekten beobachten: Wenn wir in der Arbeit mit Schüler*innen von künstlerischen Workshops sprachen, drängten sich ihre jeweiligen intrinsischen Vorstellungen, Erwartungen und auch Vorurteile gegenüber dem Begriff >Kunst in den Vordergrund. Kunst war großteils etwas, das nichts mit ihnen zu tun hatte, da ihnen in der Schule und ihrem Umfeld vorwiegend ein traditioneller Begriff im Sinne einer Hochkultur vermittelt wurde. Wenn wir uns stattdessen auf kulturelle Strategien wie DIY bezogen, beobachteten wir eine Offenheit, ohne den Ballast des Feldes Kunst (vgl. Bourdieu 1979) mitzutransportieren. Indem wir für die meisten Jugendlichen unbekannte, aber von Gleichaltrigen produzierte kulturelle Praktiken und Produktionen (zum Beispiel Zines) und ihre eigenen Interessen und Themen zum Ausgangspunkt nahmen, entstanden Anknüpfungspunkte zu ihrer Erfahrungswelt und ihrem Alltag sowie eine Atmosphäre des Interesses und der Begeisterung. Natürlich soll dies nicht heißen, dass wir den Begriff der Kunst in der schulischen Vermittlungsarbeit komplett verwerfen. Die Begegnung mit künstlerischen Praktiken kann neue Perspektiven für die Jugendlichen eröffnen. Aber wir sollten uns über die Ausschlüsse und Barrieren des Feldes Kunst in der Vermittlungspraxis klar sein und darüber, wie wir durch unser eigenes Handeln zu Co-Produzent*innen des Feldes werden. Eine Ergänzung durch das umfassende Konzept der »kulturellen Praxis« eröffnet die Möglichkeit selbstbestimmter Räume.

Ganz konkret: Wenn DIY zu einem gemeinschaftlichen Do-it-Together (DIT) wird, können Projekte ohne die Voraussetzung einer professionellen Ausbildung »zu einem offenen Experimentierfeld« (Müller 2010, 19) werden. Die Künstlerin Stephanie Müller sieht »DIY als Denkansatz, der sich eindeutig als aktionistisch und emanzipatorisch im Sinne eines Loslösens von starren Prinzipien versteht. Richtig interessant wird DIY für mich erst dann, 
wenn es zu einem Do-it-Together wird und sich nicht auf den eigenen Mikrokosmos beschränkt« (ebd., 18). Gerade durch eine solche kollaborative Arbeitsweise und in Kooperation mit Aktivist*innen und NGOs wird einer neoliberalen Vorstellung einer Anrufung von DIY als einer individualisierten Verantwortung und individueller Ermächtigung eine andere Geschichte entgegensetzt. Wenn wir kollektiv und von einem Verständnis von kultureller Praxis aus agieren, bieten sich Anschlüsse für das eigene Experimentieren und gemeinschaftliche Handlungsräume. Gleichzeitig wird die Individualisierung des Kunstbegriffs durchkreuzt.

\section{Offener Prozess und machtvolle Systeme}

Vor allem bei Making Art - Taking Part! als Sparkling-Science-Projekt wurden Widersprüche zwischen Projekt- und Verwertungslogiken und offenen Forschungs- und Vermittlungsprozessen virulent: Einerseits besteht der Wunsch und das Streben, in einem offenen Prozess mit den Jugendlichen möglichst auf Augenhöhe zu arbeiten und die Ambivalenzen ungleicher Verhältnisse in der Zusammenarbeit zu reflektieren. Andererseits sind solche Projekte in die sehr machtvollen institutionellen Systeme der Schule und Universität eingebettet und stehen in einer Logik der Drittmittelförderung, die Ergebnisse und klare Anforderungen verlangt. Diese Systemzwänge stehen konträr zu dem Wunsch, einen prozessorientierten, ergebnisoffenen Zugang in einer kollaborativen Arbeitsweise zu schaffen. Die Institution Schule spielt in der Reproduktion von sozialen Ungleichheitsverhältnissen eine zentrale Rolle, ist aber gleichzeitig ein wichtiger Ort für Transformationen eben dieser. bell hooks versteht den Klassenraum - und/oder jeden anderen Raum der Vermittlung - als einen Raum, in dem Veränderung möglich ist (1994, 110, zitiert in Kazeem-Kamiński 2016). Rubén Gaztambide-Fernandez sieht Bildung als kulturellen Prozess und Schule als einen »Ort für eine engagierte und dauerhafte kulturelle Praxis« $(2014,7)$. Der Erziehungswissenschaftler Max Fuchs fordert, dass eine kritische Pädagogik ihren Blick auf gesellschaftliche Ungleichheiten und Ungerechtigkeiten richten müsse sowie auf die wachsende neoliberale, ökonomische Denkweise, die sich auf die innere Formung des Subjekts auswirke (vgl. Fuchs 2017). Er sieht daher die zentrale Aufgabe darin, die Auswirkungen des Prozesses der Neoliberalisierung auf die Persönlichkeit zu analysieren und Widerständigkeiten vor allem mittels künstlerischer beziehungsweise kreativer Praktiken zu mobilisieren. 
Bei Making Art-Taking Part! beobachten wir, wie sehr die neoliberale Umstrukturierung unserer Gesellschaft an der Schule wirksam wird. Ein Schüler resümiert am Ende eines Videos zur Dokumentation der Projekttage: »Es war anstrengend, aber es lohnt sich. $\aleph^{11}$ Diese Aussage ist ambivalent, da sie auf die Bemühung, sich auf eine neue Erfahrung einzulassen, und ebenso auf die Subjektivierungsprozesse unter neoliberalen Vorzeichen verweist.

\section{Partizipativ und transdisziplinär forschen}

Warum nennt man etwas Forschung, das kulturelle Bildung genannt werden könnte? Was verschiebt der Ansatz der Forschung? Welche Art von Wissen wird von wem und für wen produziert? Wohin fließt das im Projekt produzierte Wissen? Wie können wir im reflektierenden Schreiben über die Erfahrungen und Prozesse Reibungen, Widersprüche und Vielstimmigkeit sichtbar machen?

Partizipative und transdisziplinäre Forschung ist durch eine offene Orientierung am Prozess, der von den Beteiligten gestaltet wird, charakterisiert. Große Unsicherheiten und Schwierigkeiten im Umgang mit Offenheit - indem keine vordefinierten Ergebnisse in Sicht sind - und mit Prozessorientierung - wo geht es hin? - bestehen bei den im Schulsystem und im Wissenschaftssystem Beteiligten gleichermaßen.

Unsere Aufgabe als Forschende bestand in der Bündelung und Fokussierung von Themen und in der Entwicklung von Workshops aus den Interessen und Wünschen der Schüler*innen heraus, um daraus Angebote zu entwickeln. Unter einem selbstreflexiven Blickwinkel betrachtet, gingen wir mit dem Anliegen in das Projekt, partizipativ zu forschen. Tatsächlich blieben die Jugendlichen in einer Rolle als Teilnehmer*innen von Fokusgruppen innerhalb eines experimentellen Settings verhaftet, sodass das Team - und nicht die Jugendlichen selbst - auf dieser Grundlage die nächsten Projektschritte gestaltete. Für einen ausführlichen Reflexionsprozess gemeinsam mit den Schüler*innen, im Sinne von lessons learned, blieb aber zu wenig Zeit. Zum Teil konnten wir noch darauf reagieren, indem wir mit einigen NMSSchüler*innen in dem erwähnten Praktikum ihre Erfahrungen reflektierten.

Auf wissenschaftlicher Ebene ist festzustellen, dass partizipative und transdisziplinäre Forschung, Aktionsforschung und communitybasierte

11 Das Video kann aufder Projektwebsite (https://www.takingpart.at/das-forschungsprojektmaking-art-taking-part/nms/projekttage-und-pr\% $\mathrm{C}_{3} \% \mathrm{~A} 4$ sentation) abgerufen werden . 
Forschung im universitären System schwierig sind und Anerkennung und Rahmenbedingungen dafür fehlen. Dies erfordert andere Strukturen und Arbeitsabläufe als in traditionell angelegten Projekten. Wir brauchen mehr Zeit und Ressourcen, die ein wirklich partizipatives Forschen und eine $\mathrm{Zu}$ sammenarbeit mit Menschen außerhalb der Universitäten möglich machen.

\section{Fazit}

Zentrales Ziel unserer Arbeit ist die Ermächtigung von jungen Menschen, in einer Demokratie zu handeln und teilzuhaben sowie gesellschaftliche Normen und Werte kritisch zu hinterfragen und zu dekonstruieren: Wie will eine Gesellschaft leben? Wie werden Ausschlüsse und Ungleichheiten hergestellt und reproduziert?

bell hooks argumentiert, dass der Prozess des Empowerments einsetzt, "wenn wir beginnen zu verstehen, auf welche Weise Herrschaftsstrukturen das eigene Leben bestimmen, wenn wir ein kritisches Bewusstsein und die Fähigkeit zum kritischen Denken entwickeln, wenn wir neue alternative Lebensgewohnheiten ersinnen und aufgrund dieses marginalen Raums von Differenz in uns Widerstand leisten " (zitiert in Johnston-Arthur/Görg o. J.). Dies ist eine enorm wichtige Arbeit für eine solidarische, offene und kritisch denkende Gesellschaft in einer lebendigen Demokratie - die aktuell, nicht nur in Österreich, untergraben wird.

\section{Literatur}

Aqra, Veronika/Huber, Laila/Smodics, Elke/Zobl, Elke (2016). Intervenieren Forschen - Vermitteln. Künstlerisch-edukative Projekte in der Kooperation Universität - Schule. Reflexionen zum Projekt »Making Art - Taking Part!«, in: p/art/icipate - Kultur aktiv gestalten, Ausgabe 7, abrufbar unter: https://www.p-art-icipate.net/intervenieren-forschen-vermitteln (letzter Zugriff: 23.7.2019).

Bourdieu, Pierre (1979). Die feinen Unterschiede. Kritik der gesellschaftlichen Urteilskraft. Frankfurt a. M.: Suhrkamp.

Engel, Antke (2007). Entschiedene Interventionen in der Unentschiedenheit. Von queerer Identitätskritik zur VerUneindeutigung als Methode, in: 
Hark, Sabine (Hg.): Dis/Kontinuitäten: Feministische Theorie, 2., aktualisierte und erweiterte Auflage, Wiesbaden: VS Verlag für Sozialwissenschaften, 285-304.

Freire, Paulo (1978 [1970]). Pädagogik der Unterdrückten. Bildung als Praxis der Freiheit, Reinbek bei Hamburg: Rowohlt.

Fuchs, Max (2017). Brauchen wir eine »Kritische Kulturpädagogik«? Eine Skizze. Kulturelle Bildung, abrufbar unter: https:/www.kubi-online. de/artikel/brauchen-kritische-kulturpaedagogik-skizze (letzter Zugriff: 23.7.2019).

Fuchs, Max (2018). Kultur für alle: Wozu? Zur Karriere einer kulturpolitischen Leitformel, in: Open Up! p/art/icipate - Kultur aktiv gestalten, Ausgabe 9, abrufbar unter: https:/www.p-art-icipate.net/kultur-fueralle-wozu/ (letzter Zugriff: 23.7.2019).

Gaztambide-Fernández, Rubén (2014). Warum die Künste nichts tun. Auf dem Weg zu einer neuen Vision für die kulturelle Produktion in der Bildung, in: Hamer, Gunhild (Hg.): Wechselwirkungen. Kulturvermittlung und ihre Effekte, München: kopaed, 51-86.

hooks, bell (1994). Teaching to Transgress. Education as the practice of freedom, New York: Routledge.

Institute for Art Education der Zürcher Hochschule der Künste (ZHdK) (2013). Zeit für Vermittlung. Eine online Publikation zur Kulturvermittlung. Im Auftrag von Pro Helvetia, als Resultat der Begleitforschung des Programms Kulturvermittlung (2009-2012), abrufbar unter: https://www. kultur-vermittlung.ch/zeit-fuer-vermittlung/ (letzter Zugriff: 10.9.2016).

Johnston Arthur, Araba Evelyn/Görg, Andreas (o. J.). empowerment, in: Online-Glossar des Thematischen Netzwerks Antirassismus, abrufbar unter: http://www.no-racism.net/antirassismus/glossar/empowerment.htm (letzter Zugriff: 10.9.2016).

Kazeem-Kamiński, Belinda (2016). Engaged Pedagogy: Antidiskriminatorisches Lehren und Lernen bei bell hooks, Wien: Zaglossus.

Klaus, Elisabeth (2006). Öffentlichkeit als Selbstverständigungsprozess. Das Beispiel Brent Sparl. In Röttger, Ulrike (Hg.): PR-Kampagnen. Über die Inszenierung von Öffentlichkeit, 3. Auflage, Wiesbaden: VS Verlag für Sozialwissenschaften, 51-74. https://doi.org/10.1007/978-3-53190076-6_3.

Klaus, Elisabeth/Zobl, Elke (2019). Kritische kulturelle Produktion im Kontext von Cultural Studies und Cultural Citizenship, in: Zobl, Elke/Klaus, 
Elisabeth/Moser, Anita/Baumgartinger, Persson Perry (Hg.): Kultur produzieren. Künstlerische Praktiken und kritische kulturelle Produktion, Bielefeld: transcript. https://doi.org/10.14361/9783839447376-003.

Langreiter, Nikola/Löffler, Klara (Hg.) (2017). Selber machen: Diskurse und Praktiken des »Do it yourself«, Bielefeld: transcript. https://doi.org/10. 14361/9783839433508.

Mörsch, Carmen (2016). Urteilen Sie selbst: Vom Öffnen und Schließen von Welten, in: Kompetenzverbund Kulturelle Integration und Wissenstransfer KIWit (Hg.): Kultur öffnet Welten, abrufbar unter: https://www. kultur-oeffnet-welten.de/positionen/position_2944.html (letzter Zugriff: 30.4.2019).

Müller, Stephanie (2007). Putting the F-Word on the Fashion Map: Wenn Mode radikal wird, in: Eismann, Sonja (Hg.): Hot Topic, Mainz: Ventil, 164-183.

Müller, Stephanie (2010). Let's do it together. Stephanie Müller aka rag"treasure im Interview mit Andrea Heinz, in: an.schläge, Heft 4, Wien, 18-19.

Reitsamer, Rosa (2013). Die Do-it-yourself-Karrieren der DJs. Über die Arbeit in elektronischen Musikszenen, Bielefeld: transcript. https://doi.org/10. 14361/transcript.9783839423233.

Reitsamer, Rosa/Zobl, Elke (2011). Queer-feministische Comics. Produktive Interventionen im Kontext der Do-It-Yourself Kultur, in: Eder, Barbara/ Klar, Elisabeth/Reichert, Ramon (Hg.): Theorien des Comics, Bielefeld: transcript, 365-382.

trafo.K (2011). FLIC FLAC* Feministische Materialien für den Kunstunterricht, Art Education Research No. 3., abrufbar unter:https://www.trafo-k. at/_media/download/trafo.K_Feministische-Materialien-f\% $\mathrm{C} 3 \% \mathrm{BCr}$ den-Kunstunterricht_2011.pdf(letzter Zugriff: 23.7.2019).

Von Unger, Hella (2014). Partizipative Forschung. Einführung in die Forschungspraxis, Wiesbaden: Springer VS. https://doi.org/10.1007/978-3658-01290-8.

Wieczorek, Wanda (2018). Zurücktreten bitte! Mehr kulturelle Teilhabe durch rationale Kulturvermittlung, München: kopaed.

Wöhrer, Veronika/Arztmann, Doris/Wintersteller, Teresa/Harrasser, Doris/ Schneider, Karin (2017). Partizipative Aktionsforschung mit Kindern und Jugendlichen, Wiesbaden: Springer VS. https://oi.org/10.1007/9783-658-13781-6. 
Zobl, Elke (2009). Cultural Production, Transnational Networking, and Critical Reflection in Feminist Zines, in: Signs. University of Chicago Press, 35(1), 1-12. https://doi.org/10.1086/599256.

Zobl, Elke (2011). »A kind of punk rock steaching machine«. Queer-feministische Zines im Kunstunterricht, in: Art Education Research: Queer und DIY im Kunstunterricht, 2(3), abrufbar unter: https://blog.zhdk.ch/ iaejournal/files/2012/o2/eJournal-AER-no-3_Zobl.pdf (letzter Zugriff: 23.7.2019).

Zobl, Elke/Drüeke, Ricarda (2012). Feminist Media: Participatory Spaces, Networks and Cultural Citizenship, Bielefeld: transcript.

Zobl, Elke/Reitsamer, Rosa mit Grünangerl, Stefanie (2012). Feminist Media Production in Europe: A research report, in: Zobl, Elke/Drüeke, Ricarda (Hg.) (2012): Feminist Media: Participatory Spaces, Networks and Cultural Citizenship, Bielefeld: transcript, 21-54. https://doi.org/10.14361/tran script.9783839421574.21.

Zobl, Elke/Drüeke, Ricarda (2016). Making Art, Making Media, Making Change! Prozesse des Queerings und des Empowerments in der Arbeit mit Jugendlichen, in: GENDER. Zeitschrift für Geschlecht, Kultur und Gesellschaft, 2, 65-82. https://doi.org/10.3224/gender.v8i2.23734.

Zobl, Elke/Huber, Laila (2016). Making Art - Taking Part! Negotiating participation and the playful opening of liminal spaces in a collaborative process, in: Conjunctions. Transdisciplinary Journal of Cultural Participation, Vol. 3(1), 1-18. https://doi.org/10.7146/tjcp.v3i1.23644.

Zobl, Elke/Klaus, Elisabeth/Moser, Anita/Baumgartinger, Persson Perry (Hg.) (2019). Kultur produzieren. Künstlerische Praxen und kritische kulturelle Produktion, transcript, Bielefeld. https://doi.org/10.14361/9783839 447376-002.

\section{Websites}

Grrrl Zine Network. http://www.grrrlzines.net Grassroots Feminism. http://www.grassrootsfeminism.net Making Art, Making Media, Making Change! https://www.makingart.at Making Art - Taking Part! https://www.takingpart.at 


\title{
Es war einmal ... die Kulturarbeit
}

\author{
Claudia Hummel
}

\begin{abstract}
In welche Geschichte schreiben wir uns ein, wenn wir heute als (kritische) Kunstvermittler*innen versuchen, demokratische Arbeits- und Aushandlungsweisen in der Gemeinschaft zu entwickeln und künstlerische Mittel dafür demokratisieren? Dieser Text möchte anhand von Beispielen aus der Geschichte des Instituts für Kunst im Kontext an der Universität der Künste Berlin und der darin mitgeschriebenen Geschichte des Begriffs der Kulturarbeit eine historisch-politische Verortung einer ästhetischen beziehungsweise kulturellen Bildungspraxis, die sich gleichzeitig als politische Bildung-als Making Democracy-versteht, nachzeichnen.
\end{abstract}

Dieser Beitrag ist aus der Perspektive einer Kunstvermittlerin geschrieben, die seit 2009 im postgradualen Masterstudiengang Art in Context am Institut für Kunst im Kontext an der Universität der Künste Berlin (UdK) unterrichtet. Mein Schwerpunkt in der Lehre trägt in der Studienordnung den Namen »künstlerische Arbeit mit gesellschaftlichen Gruppen«. Subsumiert sind unter diesem Begriff sowohl das Theorie- und Praxisfeld der Kunstvermittlung wie auch der Kulturellen Bildung.

Seit 2008 wird das Praxisfeld der Kulturellen Bildung in Berlin massiv gefördert. ${ }^{1}$ Künstler*innen, die am Institut studieren, haben dadurch relativ gute Arbeits- und Verdienstmöglichkeiten, wenn sie sich um die zur

\footnotetext{
1 Ausgangspunkt für diese Entwicklung war die Arbeitskonferenz Offensive Kulturelle Bildung in Berlin im Jahr 2006, veranstaltet vom Rat für die Künste. Im Jahr 2008 wurde in der Folge das Rahmenkonzept Kulturelle Bildung veröffentlicht, ergänzt um das Förderinstrument Projektfonds Kulturelle Bildung. Bundesweit entstanden neben bereits lang etablierten Förderprogrammen, wie beispielsweise dem Fonds Soziokultur (seit 1988) Programme wie Kulturagenten für kreative Schulen (Modellphase 2011-2015) oder Kultur macht stark (ab 2013).
} 
Verfügung stehenden Gelder bewerben. Allerdings ist diese Arbeit in gesellschaftliche Bedingungen und Erwartungen verwoben. Allerhand Aufgaben sollen durch Künstler*innen in Projekten der Kulturellen Bildung bearbeitet werden. "Ausgewogenheit zwischen kognitiver und emotionaler Entwicklung«(UNESCO 2006, 5) oder »Aufrechterhaltung einer Kultur des Friedens« (ebd.) stehen auf der Agenda sowie die Entwicklung von »Arbeitskräfte[n], die kreativ, flexibel, anpassungsfähig und innovativ sind « (ebd.). Aufmerksamkeitsökonomien und Konkurrenzkämpfe verbinden sich im wachsenden Feld der Kulturellen Bildung mit für die Künstler*innen prekärer, neoliberaler Projektemacherei. Dies führt zu Innovationsdruck, Verausgabung und Präsentationszwängen.

Weil Kulturelle Bildung aber keine neue Erfindung ist, sondern als Arbeitsfeld bereits das zweite Mal in der Geschichte - zumindest der Bundesrepublik Deutschland - einen Höhepunkt erlebt, steht im Zentrum meiner Recherchen der letzten fünf Jahre die Geschichte der künstlerisch-edukativen Arbeit in Westdeutschland und vor allem in Westberlin seit den 1970er Jahren. Aus dieser Vergangenheit lässt sich lernen. Mit dem Blick zurück nach vorn können wir uns bewusst in eine bestimmte künstlerischedukative Geschichte einschreiben, die Mär vom ewig Neuen dekonstruieren und lernen, die zeitgenössischen Anrufungen zu erkennen, bevor sie uns in Gänze regieren.

Das Institut, an welchem ich lehre, ist Teil dieser Geschichte. Im Sinne des Aufrufs Grabe, wo du stehst des schwedischen Schriftstellers Sven Lindquist (1989 [1978]) recherchiere ich seit 2015 die Geschichte dieses Instituts. Verbunden damit ist die Geschichte des Begriffs Kulturarbeit, unter welchem, zumindest bis 1998, die künstlerisch-kooperativen beziehungsweise -edukativen Praxen im Studiengang und seinen Vorläufermodellen seit dem Jahr 1976 zusammengefasst wurden. Die Materialien, die mir für diese Recherche zur Verfügung stehen, sind Publikationen und Broschüren, Dokumentationsfotos und -filme von Projekten und teils unveröffentlichte Vortragsmanuskripte des Institutsleiters von 1982 bis 2004, Prof. Helmut Hartwig. ${ }^{2}$

2 2018/19 haben zwei Ausstellungen zur nun über vierzig Jahre alten Ceschichte des Studiengangs in Berlin an der UdK und in der nCbK (neue Cesellschaft für bildende Kunst) stattgefunden. Diese Ausstellungen habe ich gemeinsam mit einem Team von ehemaligen und aktuellen Studierenden des Instituts konzipiert und umgesetzt. Die Ausstellungen 
Das Arbeitsfeld der Kulturarbeit implizierte damals das Begehren von Künstler*innen, Kunst und künstlerische Handlungsformen zu demokratisieren und demokratische Handlungen und Verhandlungen durch künstlerische Mittel zu unterstützen. Eine aus den Künsten heraus entwickelte Bildungsarbeit sollte zu Möglichkeiten der Selbstrepräsentation und der gesellschaftlichen Mitbestimmung führen. Durch Kulturarbeit reklamierten sich die Künstler*innen in die außerparlamentarische Gesellschaftsgestaltung ein.

Meine Motivation, diese Materialien einer Re-Lektüre zu unterziehen, liegt darin, zu fragen, inwiefern sie uns heute als Referenz für eine künstlerische Bildungsarbeit, die ihre politische Agenda selbst bestimmt, dienen können.

\section{Kunst als befreiende Tätigkeit - für alle ${ }^{3}$}

Die Geschichte des Studiengangs Art in Context ${ }^{4}$ begann in den frühen 1970er Jahren. In der Paulskirche in Frankfurt am Main tagte vom 4. bis 6. Juni 1971 der 1. Bundeskongress Bildender Künstler. In der Eröffnungsrede des Kongresses formulierte der bildende Künstler Gernot Bubenik: "Die Berufsgruppe der Freien Künstler, ausgebildet an den freien Abteilungen der Kunsthochschulen, angeblich frei von Vorgesetzten, Markt und gesellschaftlicher Bindung, hat vor allem die Freiheit, ihre Freiheit zu verkaufen - schlimmstenfalls berufsfremd als Angestellter der Bundespost.«Und:»Der überwiegenden Mehrheit unseres Volkes werden durch das bürgerliche Bildungsprivileg Gebrauch und Genuss von Kunst weitgehend vorenthalten. In der Aufhebung dieses Privilegs liegt zugleich die große $\mathrm{Zu}$ kunftsperspektive der künstlerischen Berufe.« (Bubenik 1970) Kunst sollte also demokratisiert und als befreiende Tätigkeit für alle eingesetzt werden. Gleichzeitig wurden damit gesellschaftliche Positionen und berufliche Handlungsräume für Künstler*innen verhandelt.

\footnotetext{
basieren auf diesem Material: https://archiv.ngbk.de/projekte/40-jahre-kunst-im-kontext/ (letzter Zugriff: 16.6.2019).

3 Teile des folgenden Textes wurden bereits auf Englisch publiziert in Hummel 2019.

4 http://www.kunstimkontext.udk-berlin.de/ (letzter Zugriff: 16.6.2019).
} 
Als Ergebnis der Konferenz wurde im Jahr 1972 vom Bundesvorstand des Bundesverbandes Bildender Künstler (BBK) eine Arbeitsgruppe mit dem Namen Kunst und Erwachsenenbildung eingesetzt. Über 20 Personen, Künstler*innen wie auch Mitarbeiter*innen von Hochschulen arbeiteten darin mit. Die Arbeit der Gruppe wurde in einem Bericht der Zeitschrift Kulturpolitik zusammengefasst (Projektgruppe Kunst und Erwachsenenbildung 1973). ${ }^{5}$ Um die Umverteilung des Zugangs zur Kunst durch Kulturelle Bildung leisten zu können, wurden Weiterbildungsmöglichkeiten für Künstler*innen gefordert, verortet in einem neu zu gründenden Weiterbildungszentrum (ebd.).

\section{Modellversuch Künstlerweiterbildung}

1976 wurde die Idee der Weiterbildung konkret: Ein sogenannter Modellversuch Künstlerweiterbildung, getragen vom BBK und der Hochschule der Künste in Berlin, finanziert durch das Bundesministerium für Bildung und Wissenschaft und dem Westberliner Senat, sollte entstehen. ${ }^{6}$ In einer Vorlaufphase vom Dezember 1976 bis Frühjahr 1978 entwickelte ein Team von Lehrenden ${ }^{7}$ ein Curriculum. Ein Gebäude wurde gesucht. Eine erfolgreiche Aushandlung mit dem Arbeitsamt ermöglichte ein Stipendienprogramm für die zukünftigen Studierenden. Die erste Gruppe an Künstler*innen begann im April $1978 \mathrm{zu}$ studieren. Das Weiterbildungsstudium dauerte ein Jahr und war kostenfrei. Untergebracht wurde der Modellversuch im Berliner Stadtteil Kreuzberg in einem solitär stehenden Altbau umgeben von Brachflächen in der Nähe der Mauer. Studierende aus den westdeutschen Bundesländern erhielten in diesem Haus sogar Wohnräume. Ein künstlerisches Internat entstand (Kunde 1981).

5 Den Bericht dieser Arbeitsgruppe kenne ich dank der Recherchen des Künstlers An-Chi Cheng, der aktuell an einer Dissertation zur Geschichte des Instituts für Kunst im Kontext arbeitet.

6 Parallel dazu gab es noch weitere Modellversuche. So etwa den Modellversuch Künstler und Schüler und ein Modellversuch mit Künstler*innen in Betrieben.

7 Der Künstler H. K. Bast, der Erziehungswissenschaftler Volker Hoffmann und die Pädagogin im Bereich Erwachsenenbildung Jutta Kunde waren ab dem 1.12.1976 als Lehrende angestellt, Christiane Zieseke war Koordinatorin und Dieter Ruckhaberle Projektleiter. 


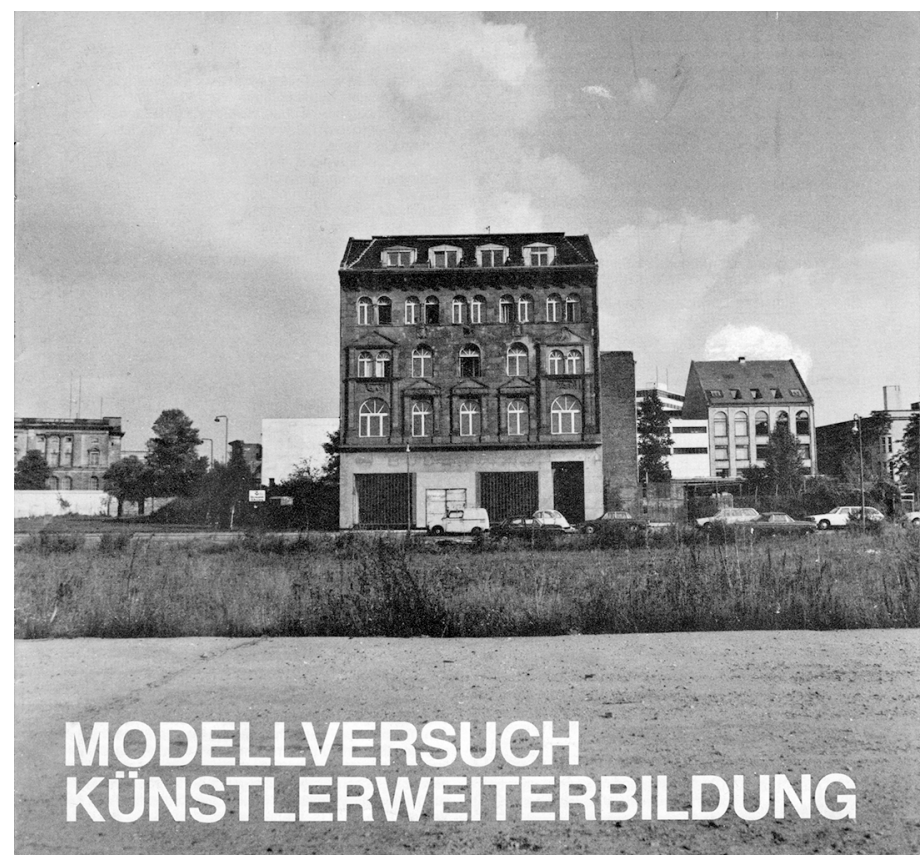

Abb. 1: Cover einer Broschüre des Modellversuchs Künstlerweiterbildung, 1980. Abgebildet ist das Haus in der Köthener Straße 44, in welchem der Studiengang und auch Studierende aus Westdeutschland untergebracht waren. Foto aufdem Cover: N. N.

Der Modellversuch Künstlerweiterbildung heißt heute Art in Context und ist ein postgradualer Masterstudiengang. Das Institut für Kunst im Kontext, an welchem er stattfindet, ist eines von vier Instituten der Fakultät Bildende Kunst der Universität der Künste Berlin.

In der Revision der Materialien zur Gründungszeit des Modellversuchs Künstlerweiterbildung wurde deutlich, dass dieser einerseits durch jahrelanges, zähes Beantragen des Vorhabens bei staatlichen Stellen entstanden und andererseits ideell von einer linken Ideengeschichte Westberlins gespeist war, die sich von den Studierendenrevolten an der Freien Universität im Jahr 1967 über den SDS (Sozialistischer Deutscher Studentenbund) bis $\mathrm{zu}$ den sogenannten K-Gruppen (Gruppierungen der Neuen Linken) spann. Diese Ideengeschichte bildet sich teilweise im ersten Curriculum des Modellversuchs ab. Die Basis waren drei verpflichtende Grundkurse: Kulturelle 
Bildung mit Erwachsenen, Kulturelle Bildung mit Jugendlichen Kunst und Gesellschaft. Ergänzt wurden diese durch die Wahlkurse (Broschüre des Modellversuchs Künstlerweiterbildung 1978 o. S.):

- Kunst und Kulturarbeit im Betrieb

- Kulturarbeit und Freizeit

- Kulturelle Bildung in der Weiterbildung

- Staat und Kulturpolitik

- Auswärtige Kulturpolitik und Demokratie

- Kommunale Entwicklung und bildende Kunst

- Verwaltung und Kulturinstitutionen

- Rechtliche Grundlagen künstlerischer Arbeitsfelder

- Bildende Kunst und Entwicklung der Arbeiterbewegung

- Geschichte der Erwachsenenbildung und Kunst

- Kunst und Kulturarbeit im Betrieb

- Kulturarbeit und Freizeit

- Geschichte der Künstlerorganisationen

- Kreativität und Gesellschaft

- Design und Kritik der Warenästhetik

- Kunst und Umwelt

- Struktur der Massenmedien

- Kulturarbeit an Haupt- und Gesamtschulen

- Kulturarbeit in der Erwachsenenbildung

- empirische Sozialforschung

- Museumspädagogik

- Kulturarbeit mit ausländischen Arbeitnehmern

- Arbeitstechniken

Parallel zu den Kursen wurde in Kooperation mit Bildungsinstitutionen für Kinder, Jugendliche und Erwachsene (Schulen, Jugendzentren, Volkshochschulen), Betreuungseinrichtungen (Krankenhäuser, psychotherapeutische Einrichtungen), Gefängnissen und Gewerkschaften und deren Bildungswerken praktisch gearbeitet.

Eine linkspolitische Agenda zeigt sich im Besonderen durch die Kooperation mit Gewerkschaften und dem damit verbundenen Versuch der Solidarisierung mit Arbeiter*innen sowie in der Kritik von Warenästhetik und Massenmedien im Sinne der Kapitalismuskritik. Die institutionell ver- 
ankerte Projektarbeit wurde durch die offene Kulturarbeit ergänzt, oft in Anbindung an sogenannte Kulturfeste, die in den 1970er Jahren in westdeutschen Städten regelmäßig veranstaltet wurden. ${ }^{8}$

\section{Die Praxis der Kulturarbeit}

Auf die Bereiche Kunst und Kulturarbeit im Betrieb und Kulturarbeit und Freizeit werde ich nun detaillierter eingehen. Beide Arbeitsbereiche ergänzten sich. Der eine versuchte, während der Arbeitszeit ein kulturelles Angebot zu schaffen, und der andere danach.

\section{Intervention in der AEG-Betriebsversammlung}
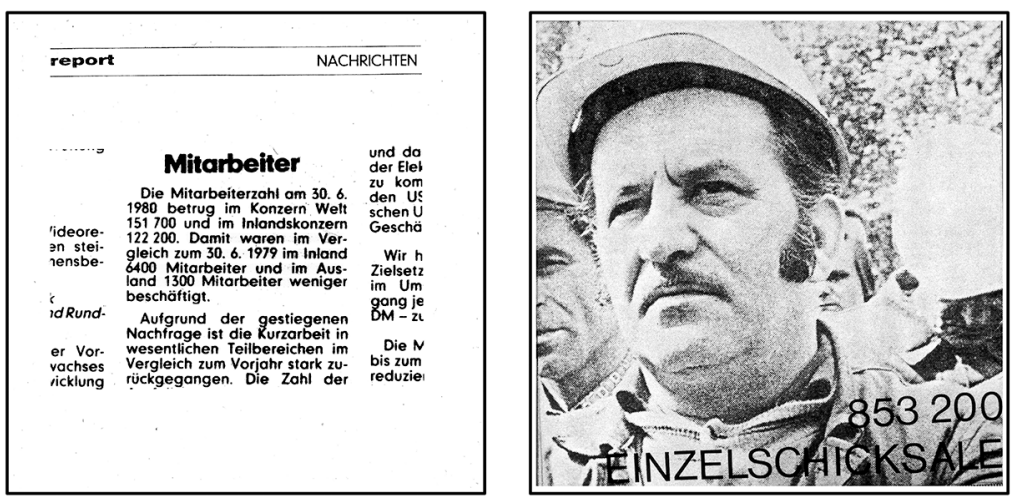

Abb. 2: Ausschnitt aus der Splitscreen-Dia-Serie für die AEG-Betriebsratsversammlung; Künstler*innen des Modellversuchs Künstlerweiterbildung in Kooperation mit dem Betriebsrat der AEG, ohne Jahresangabe

Der Künstler H. K. Bast war bereits vor seinem Wirken im Modellversuch Künstlerweiterbildung in der Stadt Karlsruhe in gewerkschaftliche Kulturarbeit involviert. Im Wahlkurs Kunst und Kulturarbeit im Betrieb setzte er diese Arbeit fort. Gemeinsam mit Studierenden initiierte er eine Zusam-

8 Kulturfeste waren damals, im Gegensatz zu den heutigen Formen von Stadtfesten, nicht ausschließlich konsumorientiert, sondern involvierten das Publikum auch in künstlerische und kunsthandwerkliche Aktionen. 
menarbeit mit dem Betriebsrat des Elektrokonzerns AEG, der im Jahr 1980 massive Rationalisierungsmaßnahmen einläutete.

Ein konkreter Anlass für eine Zusammenarbeit fand sich in der vierteljährlich stattfindenden Betriebsversammlung, die der Betriebsrat durchführen musste. Hierzu wurde ein großer Saal im Internationalen Congress Centrum Berlin angemietet. In diesem gab es zwei Großleinwände $(4 \times 4$ Meter), die den Betriebsratsvorsitzenden und seine Kolleg*innen auf die Idee brachten, diese für ihre Anliegen zu nutzen. Es sollten darauf brisante Aussprüche der Konzernleitung bildlich zitiert werden. Im Rahmen eines Theorie-Praxis-Seminars ${ }^{9}$ von H. K. Bast wurden deshalb die medialen Äußerungen des Konzerns und Berichterstattungen über ihn studiert. Eine Dia-Serie wurde erstellt. Links am Splitscreen standen Äußerungen der Konzernleitung, rechts die Perspektive der Arbeiter*innen (Bast 1981a, 138-143).

\section{Winterschule Kulturarbeit}

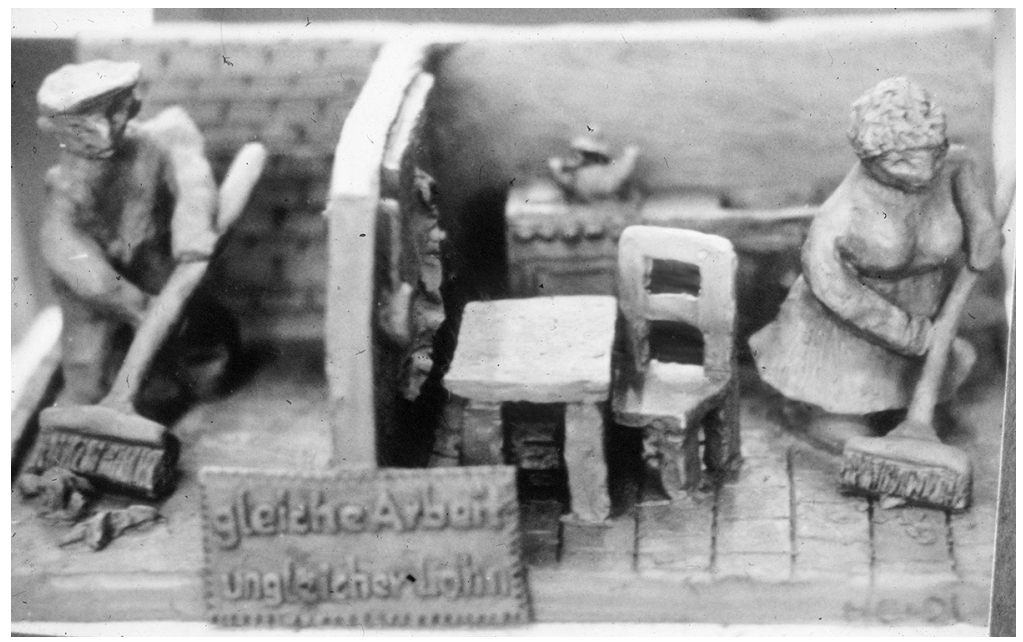

Abb. 3: Tonmodell einer Teilnehmerin der Winterschule Kulturarbeit namens Heidi; Foto: H. K. Bast, ohne Jahresangabe

9 Eine besondere Seminarform, die bis heute am Institut für Kunst im Kontext existiert. 
Ein weiteres Projekt der gewerkschaftlichen Kulturarbeit war die Winterschule Kulturarbeit. Im Dokumentationstext schreibt H. K. Bast: »Im Rahmen eines einwöchigen Seminars für Betriebsräte und Vertrauensleute aus Bielefelder Metallbetrieben und deren Familien wurden verschiedene künstlerische Techniken vermittelt. Gegenstand der Arbeit war die Darstellung von Problemen aus dem Arbeits- und Freizeitbereich, wobei hervorzuheben ist, daß es gelang, alle Familienmitglieder in die inhaltliche Arbeit und gestalterische Arbeit einzubeziehen.« (Bast 1980b, 342) In den Archivschränken des Instituts fanden sich Dias von verschiedenen Tonmodellen aus diesem Workshop. Ein Modell zeigt die Winterschule selbst, ein anderes eine Motorradwerkstatt, ein drittes beinhaltet einen durch eine Wand getrennten Raum. Links im Raum kehrt eine männliche Figur mit einem Besen, rechts eine weibliche. Auf einer Tafel auf der Vorderseite steht: "gleiche Arbeit ungleicher Lohn«.

\section{Bauvorhaben Mitmachstadt}

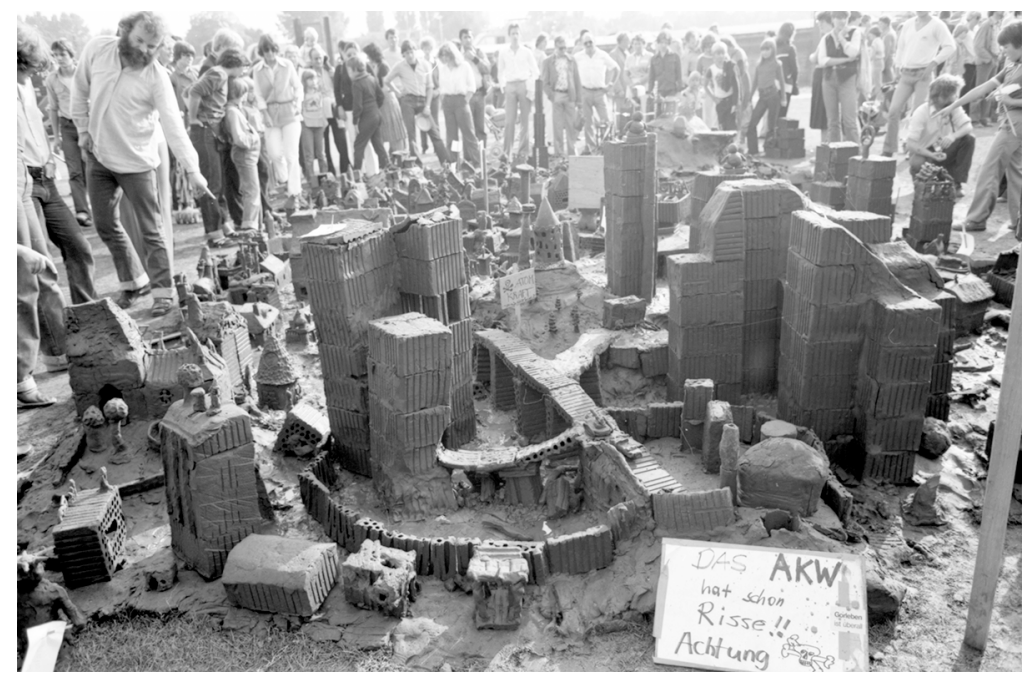

Abb. 4: Bauvorhaben Mitmachstadt, Spiellinie an der Kiellinie, Kiel; Foto: Gruppe Leut'Werk, ohne Jahresangabe

Das Bauvorhaben Mitmachstadt aus dem Arbeitsfeld Kulturarbeit und Freizeit ist ein Projekt, das in den Jahren 1979 bis 1981 in verschiedenen westdeutschen Städten stattgefunden hat. Entwickelt wurde die urbane, künstle- 
risch-partizipative Versuchsanordnung von der Gruppe Leut'Werk: Eckhart Haisch, Konstanze Schmidbauer (geb. Hedrich), Ingolf Kirsch und Gabriele Ramdohr, Studierende des zweiten Zyklus des Modellversuchs, ebenfalls begleitet von H. K. Bast. Die Versuchsanordnung bestand darin, zwischen sechs und neun Tonnen Ton in den öffentlichen Raum zu kippen und daraus über mehrere Tage hinweg zusammen mit dem Publikum eines Kulturfestes eine Modellstadt zu bauen. Anfangs entstandene ländliche Idyllen mit Giebelhäuschen wurden durch Hochhäuser oder Autobahnen unterbrochen. Die Künstler"innen ordneten Kahlschlagsanierung an, was zu Verhandlungen mit dem Publikum führte. Bürgerinitiativen wurden gebildet, um diese zu stoppen (Haisch 1981). Zwangsmodernisierung durch Kahlschlagsanierung war in Westberlin und Westdeutschland damals vielerorts ein Thema.

Mitmachstadt, AEG-Betriebsratsintervention und Winterschule zeigen, welcher Anspruch der Arbeit im Modellversuch Künstlerweiterbildung innewohnte: Künstlerische Arbeit basierte auf Kooperationen, hatte ein emanzipatives Bildungsbegehren, ein inhärentes Angebot zur Politisierung und zur Forderung von demokratischen Aushandlungsprozessen im individuellen wie gesellschaftlichen Alltag. Der Name für diese künstlerisch-gesellschaftliche Tätigkeit lautete Kulturarbeit.

\section{Kulturarbeit - eine Begriffssuche}

Kulturarbeit ist ein Kompositum aus Kultur und Arbeit. In den 1970er Jahren finden sich im Umfeld des Modellversuchs Künstlerweiterbildung neben dem bereits genannten Bericht der Arbeitsgruppe Kulturarbeit und Erwachsenenbildung einige Publikationen, die diesen Begriff untersuchen und strategisch platzieren. 1975 erschien Theorie und Praxis demokratischer Kulturarbeit der gleichnamigen NGBK-Arbeitsgruppe ${ }^{10}$ (NGBK, 1975). Die Texte im Buch arbeiten sich an den »Zwei Kulturen « nach Karl Marx ab: kapitalistische (herrschende) Kultur und die Kultur der Klasse der Arbeiter"innen. Neben Schaubildern zur Erklärung von Marx'schen Thesen werden Beispiele für demokratische Kulturarbeit angeführt. 1977 wird in der in Stuttgart vom BBK

10 Die Neue Gesellschaft für Bildende Kunst (NCBK, heute nCbK) ist ein basisdemokratischer Kunstverein in Berlin. Es gibt einige personelle Überschneidungen an Mitgliedern dieses Vereines und dem Modellversuch Künstlerweiterbildung. 
organisierten Ausstellung Künstler eine raumgreifende Installation der Entwicklung und Auslegung des Arbeitsfeldes der Kulturarbeit gewidmet (Bast et al. 1977). Im Katalog dazu taucht als Organisationsform der Kulturarbeit das Projekt auf (ebd., 102-103). ${ }^{11}$ Im Tagungsband Kulturelle Bedürfnisse der Arbeiterklasse des Instituts für Marxistische Studien und Forschungen aus Frankfurt am Main aus dem Jahr 1978 (IMSF 1978) wird unter anderem der Kulturarbeit von Organisationen der Arbeiterklasse nachgegangen.

2018 äußerte sich Helmut Hartwig, Professor der Kunstpädagogik und von 1982 bis 2004 Leiter des Berliner Studiengangs Künstlerweiterbildung, in einem Interview, dass Kultur und Arbeit im Begriff Kulturarbeit die Aufgabe hatten, sich gegenseitig zu verflüssigen. Der dabei verwendete Kulturbegriff sei damals schon an einem breiten Kulturbegriff orientiert gewesen, der am Center for Contemporary Cultural Studies (CCCS) in Birmingham entwickelt worden war. 1976 wurde das CCCS in der 24. Ausgabe »Freizeit im Arbeiterviertel« der im Zuge der Studierendenrevolten 1969 in Frankfurt am Main gegründeten Zeitschrift Ästhetik und Kommunikation zum ersten Mal im deutschsprachigen Raum vorgestellt (CCCS 1976). ${ }^{12} »$ Diese Neudefinition, letztlich auf den Begriff gebracht in Williams' ,Whole way of life`, öffnete den Weg zu einer neuen [...] Kulturanalyse« (CCCS 1976, 35). Helmut Hartwig war Gründungsmitglied dieser Zeitschrift und Mitglied des Redaktionskollektivs. Der Arbeitsbegriff stand auch hier in Bezug zur Klasse der Arbeiter*innen.

Doch der Begriff der Kulturarbeit ist älter als sein Gebrauch in der Künstlerweiterbildung in Westberlin. Der in Deutschland geborene Maler Heinrich Vogeler (1872-1942) begann 1923 nach einer Reise nach Moskau und durch Russland sogenannte Komplexbilder zu malen, die er auch als Agitationstafeln bezeichnete. Ein im Jahr 1924 gemaltes Komplexbild versah er mit dem Titel Kulturarbeit der Studenten im Sommer. In einer am Medium Collage orientierten Bildaufteilung sind verschiedene kleine Bildszenen angeordnet, bei welchen Szenen von Menschen in der Landwirtschaft, beim Schreiben, im Gespräch, beim gemeinsamen Unterricht auf der Wiese, auf einem Boot oder mit roten Fahnen auf einer kleinen Demonstration zu sehen sind. Vogeler hatte im Sommer 1924 selbst an einem Studenteneinsatz

11 Der Begriff Projekt erscheint darin wie ein Vorbote für die heutzutage überstrapazierte Organisationsform des Projekts für nahezu alle Zusammenhänge des Lebens ohne nachhaltige Strukturen.

12 Siehe hierzu auch Sternfeld (2009). 
in dem nördlich von Moskau gelegenen Twer teilgenommen und darüber ein Kapitel in seinem Buch Reise durch Russland geschrieben (Vogeler 1924). Die DDR-Kunsthistorikerin Christine Hoffmeister, die Vogelers Komplexbilder 1980 in einem Verlag im westdeutschen Bremen veröffentlichen konnte, verknüpft in ihrem bildbegleitenden Text die Szene mit den schreibenden Figuren mit dem kommunistischen Kulturprogramm, welches unter anderem die Alphabetisierung der Landbevölkerung durch Studenten zum Ziel hatte (Hoffmeister 1980, 28).

Volker Hoffmann, Lehrender am Modellversuch Künstlerweiterbildung von 1976 bis 2007 und mein Stellenvorgänger, war die Verbindung des Begriffs Kulturarbeit mit der postrevolutionären, kommunistischen Geschichte Russlands der 1920er Jahre vertraut. Er war es auch, der im Institut immer wieder zur Berliner Bildungsgeschichte derselben Zeit gearbeitet hat, beispielsweise über die Geschichte der Rütlischule in Berlin-Neukölln, die als Lebensgemeinschaftsschule in der Zeit der Weimarer Republik ein inspirierendes Vorbild für die künstlerische Arbeit mit Kindern und Jugendlichen am Studiengang bildete. Hoffmann hatte ebenfalls zu Schulstreiks im Jahr 1930 am Beispiel der Rütlischule geforscht und diese (damals wie heute) wenig bekannte Geschichte zusammen mit Studierenden in einer Ausstellung zur Schulgeschichte Berlins zwischen 1827 und 1981 in einer Schule in Berlin Schöneberg als raumfüllende Installation sichtbar gemacht (Hoffmann 1981). ${ }^{13}$

\section{Von der Kulturarbeit zum Institut für Kunst im Kontext}

In seiner Rede zum Semesterbeginn des Wintersemesters 1998 formulierte Helmut Hartwig:

"Gerade haben wir unserem Institut einen neuen Namen gegeben. Bisher hießen wir «ulturpädagogische Arbeitsstelle für Weiterbildung، oder um-

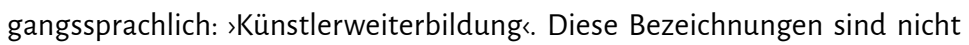

13 Auch An-Chi Cheng forscht zum Begriff der Kulturarbeit. In einem Glossar, das er anlässlich der Ausstellung 40 Jahre Kunst im Kontext. Positionen, Schnittstellen und Kommentare entwickelt hat, stellt er die These auf, »dass der Begriff ২Kulturarbeit< eine linke Herkunft hat«(Cheng 2019). 
passé. Wir haben aber einen neuen Namen darübergelegt: >Institut für Kunst im Kontexts.

Dieser Name erlaubt uns, mit einem offenen Kunstbegriff zu operieren. [...] Andererseits aber erlaubt der Kontextbegriff gerade auch die Auseinandersetzung mit [...] Kontextkünstlern: also kulturelle Arbeit in Übergangszonen und Grenzbereichen, mit Praktiken, deren Zugehörigkeit unklar ist.

Kulturelle Arbeit ... Lange Zeit war Kulturarbeit ein angesehener Begriff. Doppelt gesichert durch die Würde, das Prestige, die Anerkennung, die seine beiden Kompositionsbestandteile genossen: KULTUR und ARBEIT.

Heute sind beide Begriffe in der Krise - oder sollte ich sagen: nicht die Begriffe, sondern die bezeichneten Phänomene. [...] Der Rede von Kultur wird vorgeworfen, sie sei zu großzügig mit dem Unterschied zwischen Kunst und Nicht-Kunst/Dilettantismus/Kitsch/Massenmedien/Sozialarbeit umgegangen, habe zu sehr sozialdemokratisch nivelliert.

Und der Rede von der Arbeit: sie totalisiere im Zuge ihrer Vereinnahmung die künstlerische Praxis nach einem soziologischen Modelle, das ökonomisch, bürokratisch, zweckbestimmt gesättigt sei und in dem für die Phantasie, das Verrückte und Individuelle kein Platz sei.

Etwas ist an diesen Vorwürfen dran [...].«(Hartwig 1998, 2/3, Hervorhebungen durch den Autor im Original)

Seitdem arbeiten wir unter dem Namen Institut für Kunst im Kontext. Und der darin im Jahr 2001 modularisierte Studiengang heißt Art in Context.

Hartwig positioniert sich in seiner Rede kritisch zu den Vorwürfen und geht doch auf sie ein. Die Zeiten haben sich geändert: Distinktion scheint das Gebot der Stunde - Distinktion der Kunst von einem breiten Kulturbegriff und Distinktion des Individuums von der Masse. Der 1971 in der Zeit der sozialdemokratischen Regierung ${ }^{14}$ begonnenen disziplinübergreifenden, die Künste demokratisierenden Bewegung steht 1998 die erneute Sphärentrennung von Kunst und (politischer) Bildung gegenüber.

Neue Begriffe, wie beispielsweise die (künstlerische) Kunstvermittlung tauchten in der weiteren Geschichte des Studiengangs auf. Vom Selbstverständnis der Kulturarbeit sind darin Spuren geblieben. Die ursprüngliche Kraft der Behauptung, durch diesen Begriff ein Feld der künstlerisch-kooperativen

14 Von 1974 bis 1982 ist die SPD (Sozialdemokratische Partei Deutschlands) die stärkste Partei in der Bundesrepublik Deutschland. 
beziehungsweise der solidarischen Gesellschaftsgestaltung zu eröffnen, ist jedoch verschwunden.

\section{Fazit}

Was haben wir durch die Aufgabe des Begriffes gewonnen und was verloren? Warum ist es heute sinnvoll, sich mit der Anfangsgeschichte des Studiengangs und der Begriffsgeschichte der Kulturarbeit auseinanderzusetzen?

Wissen um den Begriff und die Formen der Kulturarbeit der 1970er Jahre kann uns heute beim Versuch, demokratische Aushandlungen mit künstlerischen Mitteln zu provozieren oder zu unterstützen, ein Referenzsystem bilden. Wissen um unser Agieren innerhalb dieser bestimmten künstlerisch-edukativen Geschichte ermöglicht es uns, in kritischer Revision des Vergangenen (so zum Beispiel der Adressierungspolitiken der 1970er Jahre), die Arbeit an einer Gemeinschaft der Gleichberechtigten fortzusetzen, ohne immer alles neu erfinden zu müssen. Und auch Kunst- und Kulturbegriffe können durch dieses Wissen im Zeitalter wieder aufkommender exkludierender Identitätspolitiken geklärt werden, um das Feld der Kulturellen Bildung für solidarische statt kompetitive gesellschaftliche (Aus-)Handlungen zu nutzen.

\section{Literatur}

Bast, H. K. (1981a). Gewerkschaftliche Kulturarbeit, in: Roloff (Hg.): Künstler und Kulturarbeit, Berlin: HdK, 119-146.

Bast, H. K. (1981b). »Winterschule Kulturarbeit«, in: Roloff (Hg.): Künstler und Kulturarbeit, Berlin: HdK, 342.

Bast, H. K./Mensch, Bernhard/Zieseke, Christiane (1977). Künstler und Gesellschaft, in: Bundesvorstand des Bundesverbandes Bildender Künstler (Hg.): Künstler, Stuttgart: BBK e. V., 13-108.

Bubenik, Gernot (1971). Eröffnungsrede, abrufbar unter: bubenikgernot. com/eig-texte.html (letzter Zugriff: 17.5.2019).

CCCS - Centre for Contemporary Cultural Studies (1976). Das Centre for Contemporary Cultural Studies (CCCS), in: Ästhetik und Kommunikation, Band 24, Freizeit im Arbeiterviertel, Frankfurt a. M.: Scriptor, 35-38. 
Cheng, An-Chi (2019). Kulturarbeit, in: Institut für Kunst im Kontext, Universität der Künste Berlin (Hg.): 40 Jahre Kunst im Kontext: Positionen, Schnittmengen, Kommentare. Glossar, Berlin: Eigenverlag, 20.

Haisch, Eckhart (1981). Praxisbericht offene Kulturarbeit, in: Roloff (Hg.): Künstler und Kulturarbeit, Berlin: HdK, 282-301.

Hartwig, Helmut (1998). Kulturarbeit, Kontextkunst, Kunst im Kontext. Berlin: Unveröffentlichtes Vortragsmanuskript.

Hoffmann, Volker (1981). »Hilfe Schule« - Kulturarbeit zwischen drei Generationen, in Roloff (Hg.): Künstler und Kulturarbeit, Berlin: HdK, 89-118.

Hoffmeister, Christine (1980). Heinrich Vogeler. Die Komplexbilder. Bremen: Worpsweder, 28-29.

Hummel, Claudia (2019). Test Model and Master's Program - An Extended History of »Art in Context« at the Institute for Art in Context at the University of the Arts, Berlin, in: Microsillons (Hg.): Motifs Incertains. Enseigner et apprendre les pratiques artistiques socialement engagées, Genf: Les Presses du Réel, 292-301.

IMSF - Institut für Marxistische Studien und Forschungen (Hg.) (1978). Kulturelle Bedürfnisse der Arbeiterklasse. München: Damnitz.

Kunde, Jutta (1981). »Fünf Jahre Modellversuch Künstlerweiterbildung«, in Roloff (Hg.): Künstler und Kulturarbeit, Berlin: HdK, 9-30.

Lindquist, Sven (1989) [1978]. Grabe, wo du stehst. Handbuch zur Erforschung der eigenen Geschichte, Bonn: Dietz.

Neue Gesellschaft für Bildende Kunst (1975). Theorie und Praxis demokratischer Kulturarbeit, Berlin: NGBK.

Projektgruppe Kunst und Erwachsenenbildung (1973). »Kulturarbeit und Weiterbildung« - Bericht der Projektgruppe »Kunst und Erwachsenenbildung« beim Bundesvorstand des Bundesverbandes Bildender Künstler, in: BBK (Hg.): Kulturpolitik, Heft 5/6, Berlin: BBK e. V., 3-13.

Roloff (Hg.) (1981). Künstler und Kulturarbeit, Berlin: HdK.

Sternfeld, Nora (2009). Schule und Institutionskritik, in: Egermann, Eva/ Pritz, Anna (Hg.): class works. Weitere Beiträge zu vermittelnder, künstlerischer und forschender Praxis, Wien: Löcker, 81-100.

UNESCO (2006). Leitfaden für kulturelle Bildung (Road Map for Arts Education), UNESCO-Weltkonferenz für kulturelle Bildung: Schaffung kreativer Kapazitäten für das 21. Jahrhundert, Lissabon: UNESCO.

Vogeler, Heinrich (1924). Reise durch Russland, Dresden: Reissner. 



\title{
Die Verhandlung demokratischer Werte als Kohärenzpraxis \\ Ein Beispiel für Hegemonie als pädagogisches Verhältnis
}

Ingo Pohn-Lauggas

\begin{abstract}
In diesem Beitrag werden die alltäglichen Aushandlungsprozesse demokratischer Werte im Lichte der Hegemonietheorie Antonio Gramscis untersucht. Es wird herausgearbeitet, inwiefern sein Begriff des Alltagsverstandes mit der Einsicht in hegemoniale als pädagogische Verhältnisse in Verbindung steht - und umgekehrt: inwiefern sich die Verhandlung von Freiheit, Gleichheit und Solidarität auch als Frage der Hegemonie darstellt.
\end{abstract}

Die im vorliegenden Band aufgeworfene Frage, wie Menschen demokratische Werte in ihrer Alltagspraxis verhandeln, lässt sich unmittelbar in Verbindung bringen mit Antonio Gramscis Analyse, dass jede philosophische Strömung ihre Spuren im Alltagsdenken der Menschen hinterlässt, sich dort buchstäblich ablagert: Dem Begriff des Alltagsverstandes (senso comune) kommt in der Hegemonietheorie bei Gramsci eine wesentliche Rolle zu, da er aus politischer Sicht die Notwendigkeit formuliert, diese eigene Weltauffassung bewusst und kritisch auszuarbeiten. In diesem Beitrag werde ich nachzeichnen, warum nicht zuletzt auch deshalb jedes Verhältnis von Hegemonie, wie es in den Gefängnisheften heißt, »notwendigerweise ein pädagogisches Verhältnis« ist (GH 10.II $\$ 44,1335) .{ }^{1}$ Wenn Gramsci nämlich in Bezug auf den Alltagsverstand von einer »Folklore der Philosophie« spricht (GH $24 \$ 4,2180$ ), nimmt er das Verhältnis von shoher und popularer Kultur

1 Aus Gramscis Cefängnisheften wird unter dem Kürzel CH mit Angabe von Heftnummer, Paragraph und Seite der deutschen Ausgabe zitiert. 
in den Blick, ohne eine abschätzige Wertung vorzunehmen. Vielmehr geht es um eine spezifische Perspektive auf jene Aushandlungsprozesse im Alltag, die auch Gegenstand dieses Buches sind, und die ich in Verbindung mit der Hegemonietheorie als Kohärenzpraxis untersuchen werde.

\section{Politik des Kulturellen}

Die Rezeption von Gramscis Hegemoniebegriff als politikwissenschaftliche Herrschaftstheorie ist ebenso verkürzt wie dessen Reduktion auf eine reine Ideologietheorie, wie sie in Ansätzen bei der Rezeption Gramscis in den britischen Cultural Studies konstatiert werden muss (Pohn-Lauggas 2013, 109 ff.; vgl. Marchart 2008, 76 ff.). Dennoch scheint es in vorliegendem $\mathrm{Zu}$ sammenhang sinnvoll, sich ihm über das Ideologische zu nähern, welches unweigerlich auf den Plan gerufen wird, wenn und zumal hier von »Weltauffassung « die Rede ist. Der Zugang der Cultural Studies erweist sich durchaus als fruchtbar, weil deren Kulturbegriff in einem unmittelbaren Zusammenhang steht mit unserem Interesse am Agieren und an den Aushandlungsprozessen in der Zivilgesellschaft, mithin an Making Democracy.

Bringen wir uns also diese Grundlagen in Erinnerung, mit Hilfe der drei »Hauptprämissen«, die Richard Johnson in den $1980 e r$ Jahren bei seiner Beantwortung der Frage formulierte, was denn »eigentlich Cultural Studies« sind:

»Die erste besagt, dass kulturelle Prozesse eng mit gesellschaftlichen Verhältnissen zusammenhängen. [...] Die zweite besagt, dass Kultur Machtstrukturen einschließt und im Hinblick auf die Fähigkeit von Individuen und gesellschaftlichen Gruppen, ihre Bedürfnisse zu definieren und zu verwirklichen, zur Produktion asymmetrischer Verhältnisse beiträgt. Aus diesen beiden Prämissen ergibt sich die dritte, die besagt, dass Kultur kein autonomes, aber auch kein von außen determiniertes Feld, sondern ein Bereich gesellschaftlicher Kämpfe und Differenzen ist.«(Johnson 1999, 141 f.)

Dies lässt sich zuspitzen auf Raymond Williams' im Rückblick formulierte Einsicht seiner Generation, dass über die Kultur »ein Weg zum Verständnis der Gesellschaft überhaupt« geführt habe (Williams 1977, 75; vgl. PohnLauggas 2017). Für den vorliegenden Zusammenhang ist an dieser Einsicht 
relevant, dass und vor allem wie ein solcher Zugang unmittelbar zur Frage des (im weitesten Sinne) Ideologischen und damit zum Alltagsverstand führt, wobei an dieser Stelle ausdrücklich festgehalten werden muss, dass es sich bei senso comune zwar um einen dem Ideologischen verwandten Begriff handelt, er damit aber keinesfalls zur Deckung kommt (vgl. Rehmann 2008, 82 ff.). Denn aus dem in der oben angedeuteten Weise politisierten Kulturbegriff resultiert der Anspruch, das Ideologische gerade nicht im Sinne eines >falschen Bewusstseins zu definieren, wie dies - mit guten Gründen freilich (ebd., 24) - dem traditionellen Marxismus zugeschrieben wird. ${ }^{2}$ Es geht vielmehr um ein soziales Sinnangebot, welches es dahingehend zu analysieren gilt, ob und wie es aufgegriffen, transformiert, reproduziert und weitergegeben wird (vgl. Pohn-Weidinger/Pohn-Lauggas 2012). In der Frage von Making Democracy also, wie etwa Freiheit, Gleichheit und Solidarität buchstäblich verhandelt werden.

Schon bei Gramsci ergibt sich die Politizität seines Kulturbegriffs aus dessen weiter Fassung. Wenn er sich für »die kulturelle Organisation« interessiert, "welche die ideologische Welt in Bewegung hält« (GH $11 \$ 12$, $1391 \mathrm{f}$., Herv. IPL), dann nimmt er ein Themenspektrum in den Blick, das von Mentalitätsgeschichte über epistemologische, philosophische, ökonomische und wirtschaftsgeschichtliche Fragestellungen bis hin zur Staatstheorie reicht. Diese Überthemen werden auch in jenen Teilen der Gefängnishefte verhandelt, die - anders als die Hefte 16 und 26 - namentlich keinen Kulturthemen gewidmet sind. All diese Notizen, kleinen Anmerkungen und großen Paragraphen werden aber durch ihre hegemonietheoretisch integrierte Perspektive auf die Politik des Kulturellen ${ }^{3}$ zusammengehalten. Für uns von Interesse ist der Umstand, dass und wie Gramsci »Kultur« in die Nähe einer

2 Vgl. etwa die berühmte Formulierung Engels' von der Ideologie als einem »Prozess, der zwar mit Bewusstsein vom sogenannten Denker vollzogen wird, aber mit einem falschen Bewusstsein. Die eigentlichen Triebkräfte, die ihn bewegen, bleiben ihm unbekannt; sonst wäre es eben kein ideologischer Prozess.« (MEW 39, 97) Für eine differenzierte, einführende Kritikvgl. Hall 2004, insbes. $16 \mathrm{ff}$.

3 Es handelt sich hierbei um eine recht gelungene Übersetzung dessen, was Cramsci als politica culturale bezeichnet, womit weder herkömmliche Kulturpolitik« noch nur politisches Handeln innerhalb des traditionell verstandenen Kulturfeldes gemeint ist, sondern womit der essentiell politische Charakter von Kultur selbst auf den Punkt gebracht wird: Cramsci gelangt, vom Standpunkt der Kulturgeschichte, zum Begriffder »kulturellen sSchöpfung «, und die sei »nicht zu verwechseln mit der künstlerischen Schöpfung, dagegen aber in die Nähe der politischen Tätigkeiten zu rücken - und in diesem Sinne kann man tatsächlich 
kohärenten »Auffassung vom Leben und vom Menschen« rückt und in ihr eine »laizistische Religion« erkennt, »eine Philosophie, die eben >Kultur< geworden ist, die also eine Ethik, eine Lebensweise, ein ziviles und individuelles Verhalten hervorgebracht hat« (GH 23\$1, 2105). Hier sind mit Philosophie, Weltauffassung und Kohärenz schon alle Schlüsselbegriffe gefallen, die uns auf das Feld des Alltagsverstandes führen, dessen Theoretisierung durch Gramsci ich an dieser Stelle kurz rekapitulieren möchte, ${ }^{4}$ um gleichzeitig die Verbindungen zu der Frage dieses Bandes herauszuarbeiten, wie Menschen demokratische Werte in ihrer Alltagspraxis verhandeln.

\section{Alltagsverstand und Making Democracy}

Zunächst gilt es das Missverständnis auszuräumen, dass der Alltagsverstand an sich positiv besetzt ist, ihm etwas grundlegend ,Vernünftiges oder gar Fortschrittliches innewohnt, im Gegenteil: Er kann bei Gramsci sowohl Ausgangspunkt kritischer und fortschrittlicher Einsichten in die Gesellschaft sein als auch Basis reaktionärer und konformistischer Haltungen. Denn im Alltagsverstand sieht er »die im Grunde genommen [...] verbreitetste Auffassung vom Leben und vom Menschen « $(\mathrm{GH} 24 \$ 4,2180)$ und er greift zu dessen Beschreibung zur Metapher der Sedimentation, wenn er festhält, dass jede philosophische Strömung »eine Ablagerung von >Alltagsverstand«" (ebd.) hinterlässt:

»es ist dies das Zeugnis ihrer geschichtlichen Wirksamkeit. Der Alltagsverstand ist nichts Erstarrtes und Unbewegliches, sondern verändert sich fortwährend, indem er sich mit in Cewohnheit übergegangenen wissenschaftlichen Begriffen und philosophischen Meinungen anreichert. Der >Alltagsverstand ist die Folklore der Philosophie [...].«(Ebd.)

Demokratische Werte wie Freiheit, Gleichheit und Solidarität, wie sie der vorliegende Band in den Blick nimmt, sind in der Alltagspraxis der Men-

von einer >Politik des Kulturellen« [politica culturale] sprechen« $(\mathrm{CH} 23 \S 7,2112)$. Vgl. einführend Haug 2011, $125 \mathrm{ff}$.

4 Ich greife hierbei auch auf ältere Textteile von mir zurück, insbes. auf Pohn-Lauggas 2007 und 2012. 
schen gewiss solche in Gewohnheit übergegangene Begriffe und Konzepte. Doch können in einer Gesellschaft zu einem gegebenen Zeitpunkt unterschiedliche Ausprägungen von Alltagsverstand parallel zueinander existieren, und mit senso comune ist jene Weltsicht zu benennen, die innerhalb einer gesellschaftlichen Gruppe die größte Verbreitung erfährt. Der Alltagsverstand leitet sich aus philosophischen Strömungen ab, die ihm vorausgegangen sind, und ist laufend Veränderungen unterworfen. Gramsci stellt also der herkömmlichen Philosophie, mithin dem, »was sich 'wissenschaftliche Philosophie nennt«, den Begriff der "popularen Philosophie« gegenüber, "die nur ein zusammenhangsloses Ensemble von Ideen und Meinungen ist" (GH $11 \$ 12$, 1379). Mit der Betonung, dass diese beiden Philosophien nicht vollständig voneinander abgekoppelt werden können, wird in den Blick gerückt, dass jede Philosophie im Alltagsbewusstsein sich verankert und eben Ablagerungen hinterlässt, woraus folgt, dass den konkreten individuellen Artikulationsformen und Deutungsmustern eine »implizite theoretische >Prämisse« (ebd., 1380) innewohnt, die aber - wie schon angesprochen nicht unmittelbar in >Ideologie $<$ zu übersetzen ist. Gramsci geht es vielmehr um die Manifestationen von Weltsicht im Alltag, um Ideologie als gelebte und habituelle gesellschaftliche Praxis. Diese »Philosophie der Nicht-Philosophen " (ebd., 1393) leitet sich, in Abgrenzung zu deterministischen marxistischen Vorstellungen, nicht aus den Interessen herrschender Klassen ab und kann daher auch nicht mit >dominanter Ideologie sleichgesetzt werden. Im Vordergrund steht vielmehr die individuelle Ausgestaltung innerhalb einer gegebenen gesellschaftlichen Deutungsstruktur: die Übersetzung von Philosophie ins Alltagsbewusstsein einzelner Menschen, wo sie dann Teil einer Alltagspraxis wird, in deren Rahmen etwa Werte wie Freiheit, Gleichheit und Solidarität laufend neu ausgehandelt werden.

Doch bleibt das Interesse bei der Feststellung, dass in diesem Sinne »alle Menschen Philosophen sind« (GH $8 \$ 204,1056$ ), nicht stehen. Aus dem Umstand, dass der Alltagsverstand nicht statisch und determiniert zu denken ist, leitet sich nicht nur die Möglichkeit, sondern in Gramscis politischer Perspektive auch die Notwendigkeit ab, ihn zu entwickeln und an sein kritisches Potenzial anzuschließen - den Alltagsverstand durch systematische Kritik selbst kritisch zu machen«. Denn, so fragt Gramsci rhetorisch:

»ist es vorzuziehen, zzu denken<, ohne sich dessen kritisch bewusst zu sein, auf zusammenhangslose und zufällige Weise, das heißt, an einer Weltauf- 
fassung steilzuhaben<, die mechanisch von der äußeren Umgebung sauferlegt ist [...], oder ist es vorzuziehen, die eigene Weltauffassung bewusst und kritisch auszuarbeiten [...]? $(\mathrm{CH} 11 \S 12,1375)$

Ein Terminus, der in diesem Zusammenhang eine zentrale Rolle spielt, ist jener der Kohärenz: die angesprochene Kritik des senso comune soll diesen nicht zuletzt kohärent machen. Dies ebnet den Einzelnen den Weg zu einem Umgang mit der Notwendigkeit, sich in einer sich wandelnden sozialen Welt zurechtzufinden, also damit umzugehen, »dass gerade durch die Tatsache des antagonistischen Werdens von Gesellschaft, des stetigen sich Veränderns der Arbeits- und Lebensweisen alle Individuen permanent zu intellektueller Kohärenzarbeit aufgefordert sind« (Merkens 2007, 171, Herv. i. O.). Die Herstellung von Einheit und Kohärenz zum Ziel der systematischen Kritik und Entwicklung des Alltagsverstandes ist bei Gramsci Voraussetzung kritischer Handlungsfähigkeit. Dieser Kohärenz-Begriff steht nicht nur gegen das Zufällige und Zusammenhanglose, das die in diesem Sinne verstandene Inkohärenz des Alltagsverstandes zunächst charakterisiert, sondern fügt der »Kohärenzpraxis« (Haug 2006, 21 ff.) noch das Element des Kritischen hinzu. Dies steht nicht nur für den Akt, die populare Philosophie selbst, sondern vielmehr »die gesamte bisherige Philosophie zu kritisieren, insofern sie verfestigte Schichtungen« $(G H$ 11 \$12, 1376) ebendort hinterlassen hat. Der Kritikfähigkeit voraus geht hier die Einsicht in den Umstand, »Produkt des bislang abgelaufenen Geschichtsprozesses [zu sein], der in einem selbst eine Unendlichkeit von Spuren hinterlassen hat «, von denen es ein »Inventar [...] zu erstellen« gilt (ebd.). Dieses Bewusstsein ist Voraussetzung von Kritik, die ihrerseits ein »Sich-Zusammen-Nehmen zur Handlungsfähigkeit« (Haug 2006, 26) erst ermöglicht.

\section{Pädagogische Verhältnisse}

Damit steht das Fenster zur Bildungsthematik weit offen. Wir kämen hier auf das Feld des »lesenden Arbeiters «, der in Brechts gleichnamigem Gedicht ${ }^{5}$ beginnt, die entscheidenden Fragen zu stellen. Dadurch wird nicht nur die Geschichtsschreibung aus Sicht der Herrschenden kritisiert, sondern mit dem Lesen auch paradigmatisch zum Thema gemacht, dass die Aneignung

5 Fragen eines lesenden Arbeiters (1935) 
kultureller Kompetenzen unverzichtbarer Teil des politischen Kampfes ist, will man seine Lebensverhältnisse kritisch begreifen und den oft zitierten »Pessimismus des Verstandes« schärfen ( $\mathrm{GH} 9 \$ 60,1117)$. Bildungsprozesse führen zur »Kunst, die richtigen Fragen zu stellen und im Material der traditionellen Geschichtsbetrachtung die Spuren der eigenen Geschichte und Wirksamkeit zu entdecken « (Jehle 2012, 1000). Doch Gramscis Auseinandersetzung mit Bildung im herkömmlichen, engeren Sinne ist nicht unmittelbarer Gegenstand des vorliegenden Beitrags, ${ }^{6}$ sondern eben das Ringen um Hegemonie und dessen pädagogische Implikationen.

Zur Erinnerung: Anliegen von Gramscis Hegemonietheorie ist es, eine Herrschaftsform zu beschreiben, die auch auf die Zustimmung der ihr Unterworfenen Bedacht nimmt und die bemüht ist, Machtverhältnisse langfristig zu festigen und gesellschaftlichen Konsens herzustellen. Die Phase, in der dies gelingt, ist für Gramsci »am eindeutigsten politisch«, da in diesem Moment »die intellektuelle und moralische Einheit bewirkt « wird (GH 13 $\$ 17,1561)$. Das Politische zeigt sich genau in diesem Prozess der Konsensbildung. Hegemonie wird im Wesentlichen in der Zivilgesellschaft verhandelt, und zur Bewahrung besagter "Einheit« schreibt sich eine hegemoniale Kraft in alle Sphären des gesellschaftlichen Lebens, in den Alltag der Menschen ein (vgl. einführend z. B. Barfuss/Jehle 2014; Opratko 2012). Mehr als ein Zustand ist Hegemonie also eine Praxis - und diese ist pädagogisch, wie eingangs schon betont, denn

»das pädagogische Verhältnis kann nicht auf die spezifisch sschulischen< Beziehungen eingegrenzt werden, durch welche die neuen Cenerationen in Kontakt mit den alten treten [...]. Dieses Verhältnis existiert in der ganzen Cesellschaft in ihrer Cesamtheit und für jedes Individuum in bezug auf andere Individuen, zwischen intellektuellen und nicht-intellektuellen Schichten, zwischen Regierenden und Regierten, zwischen Eliten und Anhängern, zwischen Führenden und Geführten [...]. Jedes Verhältnis von `Hegemonie< ist notwendigerweise ein pädagogisches Verhältnis [...].« (CH 10.II § 44, 1335)

Zumal der Alltagsverstand Ausgangspunkt kritischer und fortschrittlicher Einsichten in die Gesellschaft als auch Basis reaktionärer und konformistischer Haltungen sein kann, gilt es, wie erläutert, seine Widersprüche

6 Vgl. hierzu grundlegend Bernhard 2005; Merkens 2004; 2007; Sternfeld 2009, $53 \mathrm{ff}$. 
aufzuzeigen und ihn kritisch zu machen: Die organischen Intellektuellen ${ }^{7}$ sollen das skizzierte Streben nach Kohärenz unterstützen - und hierbei handelt es sich auch um eine Frage der Übersetzung und der Vermittlung, der Bildungsarbeit somit. Die Vorgänge im Bereich Hegemonie sind demnach (auch) Lernprozesse: Sie können Hegemonie festigen, aber auch in Frage stellen, was für den politischen »Stellungskrieg«, wie das bei Gramsci heißt, von unmittelbarem Belang ist. Bildung und Erziehung werden "nicht nur als Teil des Projektes bestehender Hegemonie, sondern als Fermente der Erosion hegemonischer Bemühungen etablierter Herrschaft, und das heißt: als Projekt der Aufkündigung des Konsenses erkennbar« (Bernhard 2005, 134).

Dies ist also die Schnittstelle zu der Einsicht, dass es bei der Verhandlung von Demokratie in der Alltagspraxis auch um Hegemonie geht: Demokratie wird, wie es im Einladungstext zu der diesem Band vorausgegangenen Tagung hieß, »auch in sozialen Beziehungen $>$ gemacht $~^{8}{ }^{-}$- und diese sozialen Beziehungen sind eben eingebettet sowohl in die informellen, privaten Bereiche als auch in die Institutionen der Zivilgesellschaft. Die Politik des Kulturellen steht für das Handeln in dieser Sphäre; Gramsci selbst spricht anders als die weite Verbreitung dieser Wortpaarung vermuten ließe - nur selten von einer skulturellen Hegemonier (was ja genau genommen ein Pleonasmus ist), doch lässt sich mit Stuart Hall das Kulturelle an Hegemonie fassen, wenn wir Kultur in diesem Sinne politisch verstehen:

»Zum einen als die Bedeutungen und Werte, die unterschiedliche gesellschaftliche Gruppen und Klassen auf der Grundlage gegebener historischer Bedingungen und Verhältnisse hervorbringen und mit deren Hilfe sie ihre Existenzbedingungen sbewältigen und auf sie reagieren; zum anderen als Cesamtheit der gelebten Traditionen und Praktiken, mittels derer diese >Übereinkunft‘ ausgedrückt und verkörpert wird.«(Hall 1999, 123)

Einzelne machen sich also einen Reim auf die Gesellschaft, in die sie eingebunden sind, bestimmen dadurch ihre politische Meinung und ihr Handeln,

7 Mit diesem Begriff verbunden ist Gramscis Einsicht, dass jede soziale Gruppe eigene ,Eliten< hervorbringt, die ihr »Homogenität und Bewusstheit der eigenen Funktion nicht nur im ökonomischen, sondern auch im gesellschaftlichen und politischen Bereich«verleihen (CH $12 \S 1,1497)$.

8 Making Democracy. Wie Freiheit, Gleichheit und Solidarität im Alltag verhandelt werden, Volkskundemuseum Wien, 27.-28. Mai 2019. 
und zwar überall, auch im Privaten. Dies verdeutlicht die Nähe dieses Hegemoniebegriffs zu Fragen der Sinnstiftung, also Weltauffassung und im spezifischen Sinn zu Ideologie.

Innerhalb von Macht- und Herrschaftsbeziehungen kommt der pädagogische Aspekt zum Tragen, wo eine Welt- und Lebensdeutung, eine bestimmte Auffassung von Demokratie etwa, sich (gegebenenfalls gegen eine andere) durchsetzt: Die Elemente des unmittelbaren Zwangs werden durch das pädagogische Binnenverhältnis überlagert. Das schafft zum einen politische Agency, heißt zum anderen aber auch, dass es auf Dauer darum gehen muss, Führungsverhältnisse zu überwinden, nicht zu verfestigen, denn »die Transformation der bestehenden (herrschaftlichen) Lehr- und Lernanordnungen [wird] zum eigentlichen Ziel emanzipatorisch-pädagogischer Praxis« (Merkens 2007, 160). 〉Gegenhegemoniale « politische Pädagogik kann und soll demnach nicht einfach die zu vermittelnden Inhalte in ihrem politischen Sinn ändern, vielmehr »ist das politisch-pädagogische Handeln auf das gesellschaftliche Herrschaftsverhältnis auszurichten, das sich über die binäre Anordnung von Führenden und Geführten, Regierenden und Regierten reproduziert « (ebd., 161). Der Kampf auf der Ebene von Ideologie und Alltagsverstand ist für Gramsci mithin ein »notwendiges Moment der Umwälzung der Praxis, die Regierten von den Regierenden intellektuell unabhängig zu machen, eine Hegemonie zu zerstören und eine andere zu schaffen« (GH 10.II \$41.XII, 1325).

Im Übrigen wohnt dem pädagogischen Aspekt des Alltagsverstandes eine Logik inne, aufgrund derer sich Gramsci entgegen eines verbreiteten Missverständnisses nicht als Stichwortgeber eines sogenannten linken Populismus eignet. Seine Forderung nach einem Anknüpfen an lebensweltliches Wissen lässt sich nicht in eine populistische Strategie überführen, die gegen den scheinbar unaufhaltsamen Rechtspopulismus in Stellung $\mathrm{zu}$ bringen ist. Denn es gibt einen entscheidenden Unterschied zum Populismus: Der beschriebene pädagogische Prozess soll zu Handlungsfähigkeit, zum zweifelnden Denken führen; es geht wie gezeigt um eine Ethik, »die einer Auffassung des Wirklichen entspricht, die den Alltagsverstand aufgehoben hat und, sei es auch noch innerhalb enger Grenzen, kritisch geworden ist « (GH 11 $\$ 12,1384)$. Populistischen Strategien geht es jedoch eben nicht um das zweifelnde Denken, sondern um einfache Erklärungen und griffige Lösungsangebote. »Die Philosophie der Praxis« hingegen, heißt es bei Gramsci, »strebt nicht danach, die >Einfachen 
zu belassen, sondern sie stattdessen zu einer höheren Lebensauffassung zu führen« (ebd., 1383). Es geht also nicht darum, Einheit auf der Ebene des Alltagsverstandes, der Folklore der Philosophie herzustellen, sondern auf der Ebene der wissenschaftlichen Philosophie: Es geht um einen »massenhaften intellektuellen Fortschritt und nicht nur einen von spärlichen Intellektuellengruppen« (ebd., 1384).

\section{Kohärenzpraxis und alltägliche Lebensformen}

Wie ich auf diesen Seiten gezeigt habe, fallen bei Gramsci Veränderung und Selbstveränderung zusammen, und Bildung kommt in dieser Verbindung ein politischer Charakter zu, mehr noch: Selbstveränderung und Bildung sind als genuin politische Tätigkeit $\mathrm{zu}$ begreifen. Hier klingt freilich die dritte der Marx'schen Thesen über Feuerbach an, die auf das »Zusammenfallen des Änderns der Umstände und der menschlichen [...] Selbstveränderung" verweist (MEW 3, 6), und in der Tat wurde die zentrale Rolle, welche diese Thesen als Ausgangspunkt von Gramscis Philosophie der Praxis spielen, schon an zahlreichen Stellen diskutiert (vgl. etwa Haug 2006, 37 ff.; in Bezug auf die Bildungsthematik Merkens 2007, 160). Ich meine allerdings, hier kommen gleich drei der Thesen zum Tragen: neben der oft zitierten elften, wonach die philosophische Interpretation der Welt mit ihrer Veränderung einherzugehen hat, die bereits zitierte dritte, die mit der Erinnerung daran, »dass der Erzieher selbst erzogen werden muss« (MEW 3, 5 f.), auf die Reziprozität des pädagogischen Verhältnisses verweist; vor allem aber These sechs, welche »das menschliche Wesen« als »das ensemble der gesellschaftlichen Verhältnisse« ausweist (ebd., 6). Denn damit wirkt das Individuum in seiner persönlichen Entwicklung zwangsläufig nach außen, weswegen für Gramsci »der Mensch wesentlich `politisch〈 ist«, weil »die ethische >Verbesserung « nie »bloß individuell« ist: Sie entwickelt »sich nicht ohne eine Tätigkeit nach außen, die äußeren Verhältnisse verändernd« (GH 10.II $\$ 48$, 1342). In Hinsicht auf Hegemonie als pädagogisches Verhältnis führt dies zur Notwendigkeit, den whole way of life, die alltäglichen Lebensformen der Menschen in den Blick zu nehmen, ihre Erfahrungen, ihr Wissen und vor allem ihre Kohärenzpraxis. Eine dieser Kohärenzpraxen ist die permanente Verhandlung von Freiheit, Gleichheit und Solidarität im Alltag. 


\section{Literatur}

Barfuss, Thomas/Jehle, Peter (2014). Antonio Gramsci: Zur Einführung, Hamburg: Junius.

Bernhard, Armin (2005). Antonio Gramscis Politische Pädagogik: Grundrisse eines praxisphilosophischen Erziehungs- und Bildungsmodells, Hamburg: Argument.

Engels, Friedrich (1968) [1893]. Brief an Franz Mehring vom 14.7.1893, in: Marx-Engels-Werke [MEW], Bd. 39, Berlin: Dietz, 96-100.

Gramsci, Antonio (1994ff.) [1929ff.]. Gefängnishefte: Kritische Gesamtausgabe, Hamburg: Argument [zit. als GH].

Hall, Stuart (1999). Cultural Studies. Zwei Paradigmen, in: Bromley, Roger/ Göttlich, Udo/Winter, Carsten (Hg.): Cultural Studies. Grundlagentexte zur Einführung, Lüneburg: Zu Klampen, 113-139. [Original 1980: Cultural Studies: Two Paradigms, in: Media, Culture and Society, Nr. 2, 57-72].

Hall, Stuart (2004). Ideologie und Ökonomie. Marxismus ohne Gewähr, in: ders.: Ausgewählte Schriften 4: Ideologie - Identität - Repräsentation, Hamburg: Argument, 8-33. [Original 1983: The Problem of Ideology. Marxism Without Guarantees, in: Matthews, Betty (Hg.): Marx - A Hundred Years On, London: Lawrence \& Wishart, 57-85].

Haug, Wolfgang Fritz (2006). Philosophieren mit Brecht und Gramsci, Hamburg: Argument.

Haug, Wolfgang Fritz (2011). Die kulturelle Unterscheidung. Elemente einer Philosophie des Kulturellen, Hamburg: Argument.

Jehle, Peter (2012). Lesende Arbeiter, in: Historisch-kritisches Wörterbuch des Marxismus, Bd. 8/I, Hamburg: Argument, 1000-1019.

Johnson, Richard (1999). Was sind eigentlich Cultural Studies?, in: Bromley, Roger/Göttlich, Udo/Winter, Carsten (Hg.): Cultural Studies. Grundlagentexte zur Einführung, Lüneburg: Zu Klampen, 139-191. [Original 1986/87: What is Cultural Studies Anyway?, in: Social Text, Nr. 16, 38-80]. Marchart, Oliver (2008). Cultural Studies, Konstanz: UVK.

Marx, Karl (1978) [1845]. Thesen über Feuerbach, in: Marx-Engels-Werke [MEW], Bd. 3, Berlin: Dietz, 5-7.

Merkens, Andreas (2004). Erziehung und Bildung im Denken Antonio Gramscis. Eckpunkte einer intellektuellen und politischen Praxis, in: Gramsci, Antonio: Erziehung und Bildung (Gramsci-Reader), Hamburg: Argument, 15-46. 
Merkens, Andreas (2007). Die Regierten von den Regierenden intellektuell unabhängig machen: Gegenhegemonie, politische Bildung und Pädagogik bei Antonio Gramsci, in: Merkens, Andreas/Rego Diaz, Victor (Hg.): Mit Gramsci arbeiten. Texte zur politisch-praktischen Aneignung Antonio Gramscis, Hamburg: Argument Verlag, 157-174.

Opratko, Benjamin (2012). Hegemonie. Münster: Westfälisches Dampfboot. [Pohn-]Lauggas, Ingo (2007). Empfindungsstrukturen und Alltagsverstand. Implikationen der materialistischen Kulturbegriffe von Gramsci und Raymond Williams, in: Merkens, Andreas/Rego Diaz, Victor (Hg.): Mit Gramsci arbeiten. Texte zur politisch-praktischen Aneignung Antonio Gramscis, Hamburg: Argument, 85-97.

[Pohn-]Lauggas, Ingo (2013). Hegemonie, Kunst und Literatur: Ästhetik und Politik bei Gramsci und Williams, Wien: Löcker.

Pohn-Lauggas, Ingo (2017). Die Politik des Kulturellen in Raymond Williams' Soziologie der Kultur, in: Horak, Roman/Pohn-Lauggas, Ingo/Seidl, Monika (Hg.): Über Raymond Williams: Annäherungen, Positionen, Ausblicke, Hamburg: Argument, 86-96.

Pohn-Weidinger, Maria/[Pohn-]Lauggas, Ingo (2012). Kohärente Erinnerung? Thesen zu Diskurs und Alltagsverstand in narrativen Quellen, in: Österreichische Zeitschrift für Geschichtswissenschaft (ÖZG): Kulturgeschichten, Nr. 2, 190-207.

Rehmann, Jan (2008). Einführung in die Ideologietheorie, Hamburg: Argument.

Sternfeld, Nora (2009). Das pädagogische Unverhältnis: Lehren und lernen bei Rancière, Gramsci und Foucault, Wien: Turia + Kant.

Williams, Raymond (1977). Was heißt »gemeinsame Kultur«?, in: ders., Innovationen: Über den Prozesscharakter von Literatur und Kultur, Frankfurt a. M.: Syndikat, 74-82. [Original 1968: Culture and Revolution: a Comment. In: Eagleton, Terry/Wicker, Brian (Hg.): From Culture to Revolution: the Slant Manifesto 1967, London: Sheed and Ward, 24-32 und 296-299]. 


\title{
Wozu lernen? = Wozu kämpfen? Pädagogik im globalen postkolonialen Raum
}

das kollektiv

\begin{abstract}
In der kritischen Bildungsarbeit mit erwachsenen Migrant*innen und Geflüchteten befassen sich Lehrende und Beratende mit brennenden Herausforderungen und Widersprüchen. Wir befragen unsere Arbeit und die Rahmenbedingungen für Erwachsenenbildungsarbeit mit Migrant*innen in der EU anhand der Formel "Wozu lernen? = Wozu kämpfen?«. Der vorliegende Beitrag gestaltet sich im Dialog mit Positionen, die wir gemeinsam mit anderen Organisationen im kürzlich erschienenen Buch Pädagogik im globalen postkolonialen Raum versammelt haben.
\end{abstract}

das kollektiv ist ein Ort der kritischen Bildungsarbeit, des Austausches, des Widerspruchs und der gemeinschaftlichen Gestaltung. Wir arbeiten in der Erwachsenenbildung mit den Schwerpunkten auf Basisbildung und dem Nachholen des Pflichtschulabschlusses mit migrierten und geflüchteten Frauen*, die am wenigsten über Privilegien verfügten und verfügen. Dieser Text spiegelt unsere Suchbewegungen nach einer Praxis wider, die zur Erweiterung der politischen und sprachlichen Handlungsfähigkeit, zur Veränderung der Ungleichheitsverhältnisse und zum Abbau strukturell bedingter Benachteiligung beitragen will. Weil wir die Welt benennen und sie so miterschaffen, können wir sie auch verändern, das lernten wir vor langer Zeit und lernen wir im Jetzt weiterhin.

Die Frauen*, die an Kursen von das kollektiv teilnehmen und Projekte, Diskussionen, Prozesse und Aktionen mitgestalten, haben nicht die gleichen Rechte wie die Mehrheit dieser Gesellschaft. Sie leben nicht unter den proklamierten demokratischen Verhältnissen, die in diesem Land für die Mehrheit gelten (sollten). Wir, das kollektiv, arbeiten im außerdemokratischen Raum, 
der gekennzeichnet ist durch die Erfahrung und wiederholte Feststellung abnehmender Rechtsstaatlichkeit (die Dokumentation rechtswidriger Asylverfahren und Asylbescheide belegt diese Aussage beispielhaft ${ }^{1}$ ), durch abnehmende soziale Sicherheit und Teilhabe und schwindende ökonomische und soziale Gerechtigkeit (denken wir an die aktuellen Bestimmungen zur Sozialhilfe: Benachteiligung von Menschen aufgrund von Sprachkenntnissen und Bildungsstand; ${ }^{2}$ denken wir an die Sicherheitspolitik: Präventivhaft exklusiv für Asylwerber"innen ${ }^{3}$ usw.). Wir arbeiten in einem Raum, in dem unter den Bedingungen des neoliberalen Kapitalismus und seines Migrationsregimes die Verfestigung dessen stattfindet, was bereits seit langer Zeit herrscht: das Außerhalb der Menschenrechte. Lernende unserer Kurse sind diejenigen, die nicht als Menschen mit Rechten gelten.

Der Name unserer Organisation steht in Verbindung mit ihrem Ursprung: maiz, dem autonomen Zentrum von und für Migrantinnen in Linz, das sich immer als Kollektiv verstanden hat. Als wir 2015 gemeinsam beschlossen, den Bildungsbereich von maiz auszugliedern, entschieden wir uns für diesen für uns und unser Handeln sehr bedeutsamen Namen. In das kollektiv arbeiten Menschen aus unterschiedlichen geografischen und sozialen Orten, die Veränderungen ungleicher gesellschaftlicher Verhältnisse anstreben.

Für diesen Text treten wir in Dialog mit Positionen, die wir - das kollektiv und maiz gemeinsam mit den Organisationen COMPA (Bolivien) und Entschieden gegen Rassismus und Diskriminierung (Deutschland) - in einem kürzlich erschienenen Buch versammelt haben (COMPA et al. 2019). Die Idee zur Publikation entstand durch die Annahme, dass angesichts zunehmender Ungleichverteilung in der postkolonialen und postnationalsozialistischen (Welt-)Gesellschaft kritische emanzipatorische Bildungskonzepte und

1 Siehe u.a.: https://www.ots.at/presseaussendung/OTS_20190108_OTS0144/bundesagenturdes-innenministeriums-gefaehrdet-chance-auf-faires-asylverfahren; https://mosaik-blog. at/asylantraege-negativ-falsch-bescheid-fehlerhaft-bfa-bvwg/; https://www.profil.at/oes terreich/roland-fruehwirth-asylanwalt-kanzlei-ende-10894507 (letzter Zugriff: 28.8.2019).

2 Siehe v. a. Abschnitt»Arbeitsqualifizierungsbonus « unter dem Link: https://www.sozialmi nisterium.at/site/Soziales_und_KonsumentInnen/Sozialhilfe_Mindestsicherung/Voraus setzungen/ (letzter Zugriff: 28.8.2019).

3 Siehe u.a.: https://www.derstandard.at/story/2000098828923/praeventive-sicherungshaftdas-grosse-misstrauen-gegen-kickl-co; https://www.addendum.org/news/sicherungshaft/ (letzter Zugriff: 28.8.2019). 
-praxen notwendig sind. Wir stellten uns und den eingeladenen Organisationen, Projekten und einzelnen Autor*innen die Frage, wie progressive Pädagogik reflexiv und intervenierend Impulse für gerechtere Verhältnisse, insbesondere in Bezug auf Bildung, geben kann. Das Buch gliedert sich in drei Bereiche: Praxis, Geschichte und Theorie. Einer der theoretischen Beiträge ist ein Essay von Silvia Rivera Cusicanqui (2019), den wir hier im Zusammenhang mit dem Thema des Ausschlusses aus demokratischen Strukturen einbringen. Aber zuvor möchten wir den Hintergrund der Entscheidung für die Formel im Titel dieses Textes erläutern: Wozu lernen? = Wozu kämpfen?«

Wenn wir im Dialog mit Praxen und Positionen aus dem Globalen Süden etwas lernen wollen, sollte die Beantwortung der Frage »Wozu lernen?« im Vorfeld geklärt werden. Denn wie kann es uns gelingen, dass der durch das Lernen entstandene Profit sich nicht wieder vor allem auf der Seite derjenigen mit mehr Macht ausgestatteten Subjekte im Globalen Norden niederschlägt? Die Formel suggeriert, dass erst durch die Beantwortung der zweiten Frage "Wozu kämpfen?« die erste beantwortet werden kann. Die Verbindung der Kämpfe - so die Annahme - würde das Lernen im Dialog mit widerständigen Akteur*innen in Lateinamerika zu einem herausfordernden Impuls für das weitere Denken und Handeln im Kontext unserer kritischen Bildungsarbeit in Europa werden lassen. Eine Verbindung der Kämpfe würde unter anderem bedeuten: Intensivierung von transnationalen feministischen und antirassistischen Kooperationen und Bündnissen, Austausch, gemeinsame Analysen, koordinierte Aktionen, Unterstützung, Solidarität, eine lokal situierte Praxis in Kohärenz mit dem Ansatz der Verbindung der Kämpfe; und vor allem, wie Cusicanqui in ihrer Kritik am akademischen Feld anmerkt, Ideen nicht - wie im Weltmarkt der Waren - wie Flüsse aus dem Süden in den Norden fließen zu lassen, damit sie sich dort in Seitenarme großer Gedankenströme verwandeln. Denn so kehren die Ideen als »erbrochener Mischmasch in Form eines Endproduktes« zurück in den Süden (Cusicanqui 2019, 274).

Das ist die erste Bedeutungsebene der Formel. Eine zweite Ebene führt uns zu Paulo Freires Konzeption kritischer Erwachsenenbildung, welche diese als Ort der Organisierung einer kollektiven Veränderungspraxis betrachtet. Kritische Bildungsarbeit würde demnach zu organisierten Kämpfen und Strukturveränderungen führen (vgl. Streck et al. 2008, 139 ff.). Ausgehend von dieser Interpretation der Formel versuchen wir uns mit Lernenden und mit Mitarbeiter*innen in das kollektiv über unsere Arbeit auszutauschen. Wobei es uns in diesem Lernprozess auch um die Identifizierung von sowie 
um die Auseinandersetzung mit internen Widersprüchen, Leerstellen, Versäumnissen und um einen bestimmten Umgang mit diesen geht, sodass daraus produktive und widerständige Kräfte - anstatt Lähmungs- und Überforderungserscheinungen - entstehen.

Es werden im Verlauf dieses Beitrags einige Aussagen aus Gesprächen mit Kursteilnehmerinnen* wiedergegeben und kommentiert. Zunächst beschäftigen wir uns ausgehend von der Formel im Titel mit Positionen, die im Buch vertreten werden - vor allem ausgehend von der Lektüre des bereits erwähnten Essays von Cusicanqui.

\section{Die trügerische Inklusion und der Archaismus der Eliten}

Silvia Rivera Cusicanqui wurde 1949 in La Paz (Bolivien) geboren, ist Soziologin und Aktivistin in der kataristischen Bewegung, in der Coca-Bäuer*innenbewegung und in der Befreiungsbewegung. Sie orientiert sich am Anarchismus und an dekolonialen Praktiken und Theorien und gilt als eine der bedeutendsten radikalen Denker*innen der lateinamerikanischen Gegenwart (vgl. Garbe et al., 2018, 7 f.). Gemeinsam mit anderen indigenen und mestizischen Intellektuellen gründete sie 1983 die Werkstatt der oralen Geschichte der Anden (Taller de Historia Oral Andina), eine unabhängige Gruppe, die sich den Themen der Oralität, Identität und sozialen indigenen und populären Bewegungen (vor allem in der Region der Aymara im nördlichen Bolivien und südlichen Peru) widmete. Über drei Jahrzehnte war sie Soziologieprofessorin der Universidad Mayor de San Andrés in La Paz, seit 2014 ist sie emeritiert. Sie arbeitet zudem als Gastprofessorin an verschiedenen Universitäten in den USA, Argentinien, Ecuador und Frankreich (COMPA et al. 2019, 327; Cusicanqui 2010; Garbe et al. 2018).

In ihrem Essay Ch'ixinakax utxiwa. Eine Reflexion über Praktiken und Diskurse der Dekolonisierung beschäftigt sich Cusicanqui mit der Inklusion von Indigenen unter dem Zeichen des Multikulturalismus und übt eine radikale Kritik an Dekolonisierungstheorien und an akademischer Wissensproduktionen im Allgemeinen. Der Essay wurde 2010 auf Spanisch veröffentlicht und ist 2018 ins Deutsche übersetzt worden. ${ }^{4}$ Die Autorin berichtet darin,

4 Der Essay ist in unserem Buch erschienen und in einem weiteren, das mehrere Texte der Autorin beinhaltet (vgl. Garbe/Cárdenas/Sempertegui 2018). 
dass sie anhand ihrer Forschungsarbeiten im Vorfeld des Erscheinens des Buchs Oprimidos pero no vencidos (dt. Unterdrückt, aber nicht besiegt) im Jahr 1986 verschiedene Momente der Herrschaft in Bolivien (die koloniale, die liberale und die populistische Herrschaft) analysierte. Sie stellt im Rahmen dieser Arbeit fest, dass seit dem 19. Jahrhundert liberale und modernisierende Reformen zu einer bedingten Inklusion von Indigenen, zu einer »beschränkten Staatsbürgerschaft zweiter Klasse« in Bolivien führten (Guha 2000 zit. n. Cusicanqui 2019, 266). In diesem Zusammenhang schreibt sie über Rekolonisierung und über die Etablierung einer finanzbasierten Herrschaft, die auf Privilegien der Kolonialmacht gründete. Und sie merkt an, dass in der Gegenwart die Rhetorik der Gleichheit und der Bürgerrechte eine Karikatur sei, die es vermag, politische und kulturelle Privilegien zu verbergen. Auf diese Weise könnten sich koloniale Strukturen der Unterdrückung weiterhin reproduzieren.

Cusicanqui kritisiert Politiken und Maßnahmen, die im Einklang mit Multikulturalismusdiskursen umgesetzt werden. Multikulturalismus beschreibt sie im Kontext von Bolivien als den "tarnenden Mechanismus schlechthin für neue Formen der Kolonisierung " (Cusicanqui 2019, 269). Dem Archaismus der bolivianischen Eliten stellt sie die Modernität der Indigenen gegenüber, welche durch Multikulturalismusdiskurse geleugnet und nicht anerkannt wird. Die Modernität der Indigenen begründet sie anhand historischer und politischer Argumente. Die Indigenen wären modern, weil sie das Problem des Überlebens anhand von drei Eigenschaften beziehungsweise Praxen lösen würden: Sie seien kapitalistisch, selbstverwaltet und könnten in mehreren Welten leben, Codes ändern sowie Grenzen überschreiten. Für Cusicanqui ist es notwendig, essenzialisierende Diskurse zu bekämpfen, die auf Forderungen von Indigenen nach politischer Teilhabe mit einem Rekurs auf die Idee des Ursprungs reagieren. Dadurch würden die indigenen Forderungen strategisch benutzt und ihre dekolonisierende Kraft neutralisiert (vgl. Cusicanqui 2019, 270 f.).

Inwieweit können wir hierzulande ebenso über eine Tradition »trügerischer Inklusion« von Migrierten und Geflüchteten reden? Rekurrierend auf kulturalisierende Zuschreibungen bewirkten staatliche Politiken in Österreich seit den ersten Migrationsbewegungen der 1960er Jahre die Entstehung und Ausweitung gesellschaftlicher Segregation von Migrant"innen. Politische, soziale und ökonomische Benachteiligung der »Anderen« sowie die Verfestigung ihrer Positionierung außerhalb der privilegierten Sphären 
ließen sich demokratiepolitisch kontinuierlich und vermehrt als konsensfähige Ansätze und Maßnahmen umsetzen. Die kulturalisierenden Diskurse der politischen »Mitte« artikulierten sich in enger Verbindung mit neoliberalen Politiken, die wiederum gewaltvolle Verhältnisse im Bereich der Migrations-, Asyl-, Arbeitsmarkt- und Sozialpolitiken legitimierten und verstärkten.

\section{Einblick in Gespräche mit Kursteilnehmerinnen*}

»Allein die Tatsache, dass wir im Kurs sind, ist ein Kampf. ${ }^{5}$

Das Unterrichtszimmer ist einer der Räume, den wir in unserer pädagogischen und politischen Praxis bewohnen. Ein Raum, in dem ein Austausch der Erfahrungen aller Beteiligten aus unterschiedlichen Gewaltkontexten stattfindet. Ein Raum, der im Bewusstsein darüber, dass er nicht außerhalb von Gewaltverhältnissen existiert, im Streben danach gestaltet wird, Neues entstehen zu lassen. Ein Raum, wo die Körper und Stimmen derjenigen, die den Lernprozess unterbrechen mussten, weil sie in einem anderen Raum, in einer Zelle, auf ihre Abschiebung zu warten, in ihrer Abwesenheit präsent sind.

»ch lerne zu protestieren.«

In diesem Raum, in dem wir uns einer kritischen pädagogischen Praxis verschrieben haben, entstehen Positionierungen, Appelle, Forderungen, Infragestellungen, Verzweiflung, Hoffnungsschimmer und Kämpfe, deren Ausgang sich nicht in den Kategorien Gewinn oder Verlust fassen lässt. Von einer Kursteilnehmerin" wurde es wie folgt beschrieben:

„Das zeigt, dass wir starkes Selbstvertrauen haben. Dass wir wissen, dass wir es können und dass wir durchkommen; wir kämpfen einfach. Das ist etwas, was sein muss. Und wenn es sein muss, dann machen wir es.«

Im Juni 2018 verfasste eine Gruppe afghanischer Frauen* in das kollektiv einen Text, mit dem sie die Öffentlichkeit adressierten. Darin ist zu lesen:

5 Dieses und alle folgenden Zitate ohne Quellenangabe stammen aus Gesprächen, die bei das kollektiv im Zeitraum zwischen 8.5. und 17.5.2019 geführt wurden. 
»Wir wollen das Recht auf Leben. Das Recht, unser Leben zu gestalten [...] Ich bin hier, damit meine Kinder eine bessere Zukunft haben, damit meine Kinder eines Tages jemandem etwas vorlesen können, eine Möglichkeit, die ich nie hatte.«(Afghanische Frauen in das kollektiv 2018)

Eine der Frauen*, die den Raum bewohnen und mitgestalten, stellt eine weitere Formel auf: »Bildung = Reflexion«. Sie meint:

»Lernen ist gleich kämpfen, weil wir damit eigentlich dem Analphabetismus den Kampf ansagen. Wenn du lernst, kämpfst du dagegen, damit du nicht mehr Analphabetin bist.«

Sie weist darauf hin, dass Bildungsprozesse ausschlaggebend für die Ermöglichung der Reflexion und der kritischen Hinterfragung eigener beziehungsweise aufoktroyierter Normalitätsvorstellungen seien. Der gemeinsame Kampf gegen den Analphabetismus ist aus ihrer Sicht ein Kampf um eine andere, reflektierte und offene Gesellschaft, wie sie in einem anderen Gespräch erläutert.

Der gedankliche Entwurf dieser Gesellschaft gestaltet sich, angesichts der Kräfteverhältnisse, vorsichtig. Aus dem außerdemokratischen Raum hören wir:

»In einer Demokratie lerne ich, was man nicht darf und dass die Österreicher*innen mehr dürfen.«

Es wird entgegengehalten:

»Ich bin der Meinung, dass wir auch das Recht zu wählen haben sollen.«

Das Recht auf freie Meinungsäußerung wird als ein zu erkämpfendes Recht und nicht als Gegebenheit gesehen:

»Protestieren ist freie Meinungsäußerung in der Demokratie.»

Zum Thema Freiheit betont eine Teilnehmerin*, dass Entscheidungen nicht frei, sondern von unterschiedlichen Ausprägungen der gewaltvollen Verhältnisse bestimmt sind: 
»/ch lerne, was ich nicht kann/soll/darf, auch wenn ich frei bin.«

Im oben erwähnten Text der afghanischen Frauen* lesen wir zum Thema Gerichtsbarkeit:

»Wir warten 2, 3, 4 Jahre auf einen Interviewtermin und hoffen. Beim Interview werden wir oft eingeschüchtert und aggressiv adressiert. Wir haben nicht das Cefühl, dass es jemanden überhaupt interessiert, warum wir geflohen sind. Wir haben das Gefühl, dass die negative Entscheidung schon feststeht, noch bevor wir unseren Fluchtgrund benennen. Unser Leben wurde von Angst bestimmt. Und wir haben das Cefühl, dass uns beim Interview noch mehr Angst gemacht werden soll.

Mit viel Mühe arbeiten wir gegen diese Angst, mit der Zeit schaffen wir es, ein bisschen weniger Angst zu haben. Aber das ist offensichtlich nicht erwünscht.«(Afghanische Frauen in das kollektiv, 2018)

Und um nochmals zur Frage nach Gleichheit zurückzukommen:

»Ich lerne, dass das Wissen der Anderen mein Leben bestimmt.«

Diese Aussage lenkt unseren Blick in Richtung der aktuellen Diskurse, die feministische Positionen, Forderungen und Anliegen vereinnahmen, um eine »Andersheit« herzustellen, die im Gegensatz $\mathrm{zu}$ einer vermeintlichen europäischen »Normalität der Gendergerechtigkeit« als rückständig gekennzeichnet wird. Dies zeigt sich exemplarisch in der Implementierung und Legitimierung von Wertevermittlung an Migrant*innen und Refugees, dem Nachweis der »westlichen Orientierung« im Rahmen der Befragungen im Asylverfahren und der »fehlenden westlichen Orientierung « als Ablehnungsgrund. Eine Modernität, deren Konzeption und Definition den Eliten im Globalen Norden obliegt, wird als Imperativ aufgezwungen, während bis vor kurzem regierende Politiker*innen Nazi-Parolen schreien (vgl. Mauthausen Komitee Österreich 2017). Es soll an dieser Stelle allerdings festgehalten werden, dass die politischen Ansätze, die zu Aberkennung der Würde und Integrität der Menschen in diesem Land und anderswo führen, nicht nur einer politischen Partei zugeschrieben werden können. Sie stellen ein Gebilde dar, über das es einen politischen Konsens zu geben scheint. Oder 
wie es die zapatistischen Frauen in einem offenen Brief »an die Frauen, die auf der ganzen Welt kämpfen«, formulieren:

"Sie möchten, dass wir ihre Hilfsarbeiterinnen werden, ihre Mägde, dass wir unsere Würde um einige Celdstücke im Monat verkaufen. Denn diese Kapitalisten und jene, die in den neuen schlechten Regierungen sitzen und ihnen gehorchen, glauben, dass wir Geld wollen. Sie können nicht verstehen, dass wir die Freiheit wollen, sie verstehen nicht, dass das Wenige, das wir erreicht haben, wir durch unseren Kampf erreicht haben, ohne dass jemand darüber berichtet, ohne Fotos, ohne Interviews, ohne Wahlen, ohne Museen und ohne Lügen. Die verstehen nicht, dass alles, was sie `Fortschritt nennen, eine Lüge ist, und dass sie nicht einmal fähig sind, die Sicherheit der Frauen zu gewährleisten. Diese werden weiterhin geschlagen, vergewaltigt und ermordet, dort in ihren fortschrittlichen oder reaktionären Welten. Wie viele Frauen wurden in diesen progressiven oder reaktionären Welten ermordet, während du diese Worte liest, compañera, Schwester?

Vielleicht weißt du es, aber wir sagen es dir deutlich, hier, im zapatistischen Territorium wurde seit vielen Jahren keine einzige Frau ermordet. Aber natürlich, sie sagen, dass wir die Rückständigen sind, die Dummen, die Unwichtigen.«(Die zapatistischen Frauen 2019)

Der Ausschnitt aus dem Brief soll keinesfalls zu Vergleichen verleiten. Die zapatistische Bewegung ist eingebettet in einer bestimmten politischen Organisierungspraxis, die sich von unserer unterscheidet. Und doch war und ist die zapatistische Bewegung eine Referenz für uns, um die geteilten politischen Positionen, Ansätze und Kämpfe zusammenzudenken, ohne die Unterschiede zu ignorieren. Eine Teilnehmerin* von das kollektiv hält fest:

»Lernen ist gleich kämpfen, weil wenn wir lernen, dann können wir unsere eigenen Probleme lösen und nicht von anderen abhängig sein.«

Die Kämpfe beginnen dort, wo Veränderung möglich erscheint. Bildung als ein Weg zur Begegnung der Herausforderungen, als Kampf um die Zukunft.

»Wenn wir nach Hause gehen, erzählen wir, was wir gelernt und geschafft haben, und diese Idee übernehmen auch unsere Kinder und unsere Kinder 
werden auch Kinder haben und so bildet sich ein Kreis und wir ändern auch was.«

Das Lernen ist also eingeschrieben in das Kämpfen und vice versa. Ausgangspunkt sind unter anderem die individuelle Situation und die wahrgenommenen Handlungsmöglichkeiten. Gerade in den »alltäglichen Handlungszusammenhängen« schöpft nach Kaloianov (2017, 149) migrantische Kritik ihre Stärke: »Nichts fundiert kritisches Denken und kritische Aktivität stärker und verlässlicher als die Normalität des Lebens, wenn einem/einer diese Normalität versagt bleibt.«

Die Kämpfe, die aus Kritik abgeleitet werden, sind unterschiedlich, darauf weisen auch die zapatistischen Frauen in Hinblick auf feministische Kämpfe hin. Doch die utopischen Horizonte berühren sich:

»Was wir aber sehr wohl verstehen ist, dass wir für unsere Freiheit kämpfen und dass wir jetzt kämpfen müssen um sie zu verteidigen, damit unsere Töchter und Enkelinnen niemals die Ceschichte des Schmerzes unserer Großmütter erleiden müssen.

Wir müssen kämpfen, damit sich die Ceschichte nicht wiederholt, als wir nur dafür auf die Welt kamen, um zu kochen und Kinder zu gebären, damit wir dann sehen mussten, wie sie unter Demütigung, Verachtung und Tod heranwachsen mussten.

Wir haben die Waffen nicht ergriffen, damit dieselbe Situation wieder zurückkehrt.«(Die zapatistischen Frauen 2019)

Wir, das kollektiv und maiz, haben nicht, oder nur metaphorisch gesprochen, die Waffen ergriffen. Immer wieder tauschen wir uns zu den Herausforderungen in Hinblick auf die Organisierung einer politischen Praxis aus. Dabei reflektieren wir die Entwürfe dieser Organisierung aus unseren sozialen Positionierungen heraus. Und wir fragen uns, wie diese Räume, in denen Kritik und Protest formuliert werden, in denen Normalitäten infrage gestellt werden, in denen individuellen Erfahrungen auf ihre kollektive Ebene hin überdacht werden, verstärkt zur Organisierung einer politischen Praxis gestaltet werden könnten. 


\section{Aus den Gesprächen zwischen den Lehrenden}

In dem physisch abgegrenzten Raum unserer Organisation schließen sich Lehrende, meist geflüchtete und migrierte Frauen*, mit ihren differenzierten und zu differenzierenden Gewalterfahrungen zusammen, um gemeinsam mit den Lernenden (meist geflüchteten Frauen*) zu versuchen, Umgangsund Widerstandsformen in Hinblick auf die gewaltförmigen Lebensbedingungen zu entwickeln.

Die Formel »Wozu lernen? = Wozu kämpfen? « bietet sich an, um unsere Kämpfe als Organisation zu definieren und zu reflektieren, und führt uns zu weiteren Rahmenbedingungen unserer Arbeit, beispielsweise der technokratischen Logik, die sowohl Diskurse um Migrationsmanagement (Schütze 2016) als auch unsere direkte Arbeit im Bereich der Projektfinanzierung und -abrechnung prägt. Auf Ebene der Organisation sind wir damit konfrontiert, dass die Expert*innen, die die Effizienz und Nachvollziehbarkeit unserer Arbeit bewerten und beurteilen, Wirtschaftsprüfer*innen sind. Das Erlernen eines strategischen Umgangs damit, unter anderem die Aufstellung eines Verwaltungsbereichs, der nicht angreifbar ist, wird in den Gesprächen mit den Kolleg*innen thematisiert:

»Misstrauen wird größer gesprochen als Zutrauen, alles muss bewiesen, belegt werden; wir lernen damit umzugehen.«

Doch wozu dieses Lernen? Ähnlich wie im Gespräch mit den Teilnehmenden betonen Kolleg*innen die Wichtigkeit dieses gemeinsamen Raums:

»Die Organisation zu erhalten, das ist ein Kampf.«

Die Organisation wird auch als ein Raum beschrieben, in dem wir über uns lernen:

„Wir haben hier den Raum für Reflexion, für das Hinterfragen unserer Arbeit. Welche Position habe ich hier, bin ich Rassistin? Das können wir uns fragen, überlegen, in unserem Alltag hinterfragen.« 
»Was ich gelernt habe: kritisches Denken, habe früher nicht viel hinterfragt, nicht viel kritisch beobachtet; durch die Arbeit im Kurs hinterfragt man um so mehr.«

»Wir lernen im Sozialen, im mitmenschlichen Bereich, Großzügigkeit, Achtsamkeit, im wissenschaftlichen Bereich, Theorie, Fortbildungen als Werkszeuge für Unterricht.«

»Wir lernen durch das (hinter-)fragende Lehren.»

Auch der Umgang mit den Paradigmen, die immer stärker den Unterrichtsraum bestimmen, soll erlernt werden, um die Kämpfe weiterzuführen:

»Kämpfen gegen den kapitalistischen Gedanken, Leistungsdruck.»

In diesem Spannungsfeld zwischen sich durchkreuzenden Gewaltverhältnissen verharren und handeln wir, geleitet von der Vorstellung, dass es möglich und notwendig ist, einen strategischen Umgang mit bürokratischen Erfordernissen und neoliberalen Paradigmen zu finden. Wobei es nicht nur um die Existenzsicherung der Organisation, sondern vor allem darum, sich als politisch organisierte Migrant*innen und Geflüchtete immer neu zu erfinden, um den brennenden Herausforderungen zu begegnen und um weiterhin für die Transformation der ungerechten Verhältnisse zu kämpfen.

Im Wissen, dass die Forderungen, die durch kritische Bildungsarbeit formuliert und im Rahmen von Protestaktionen artikuliert werden, meist nur bis in den »Vorraum« der Paläste - wie Cusicanqui politische Institutionen wie das Parlament bezeichnet (Cusicanqui 2010, Übersetzung der Autorinnen*) - dringen, entsteht die Notwendigkeit der politischen Arbeit, unter anderem im Sinne der Transformation bestehender institutionalisierter Räume der Entscheidungsmacht. Im aktuellen bildungspolitischen Kontext herrscht der Imperativ der Kompetenzorientierung, der nicht zur Förderung kollektiver Prozesse der kritischen Wissensproduktion und des kritischen Denkens beiträgt. Stattdessen werden wir erst mit Projekten beauftragt, wenn wir uns bereit erklären, individualisierte Formen des Empowerments oder der Selbstwirksamkeitsüberzeugung zu fördern, wenn wir Skills vermitteln, die zu einer Inklusion in prekäre Systeme und nicht zur Organisierung und Widerstand beitragen (vgl. Gohn 2019, 168). 


\section{Fazit}

Aus dem Dialog mit den Positionen aus den Globalen Süden bestätigt sich für uns die Gewissheit über die Notwendigkeit der Verbindung der Kämpfe und der Fortsetzung und Intensivierung unserer widerständigen und erfinderischen Handlungen auf der Ebene der Mikropolitik. So formuliert Cusicanqui:»Mikropolitik ist eine moralische Pflicht, um zu verhindern, dass die Zukunft der Makropolitik unser Leben zerstört.« (Cusicanqui 2010, Übersetzung der Autorinnen*)

Für den lateinamerikanischen Kontext betont Cusicanqui, dass es eine Dekolonisierung durch konkrete Praktiken bedarf, um einen Bruch mit den innergesellschaftlichen kolonialen Verhältnissen zu vollziehen. Es reiche nicht, diese nur diskursiv oder theoretisch einzufordern. Für Cusicanqui gibt es keine Dekolonisierungstheorie ohne dazugehörige Praktiken (vgl. Cusicanqui 2019, 270).

Praxis wird von maiz und das kollektiv als Aktion und Reflexion verstanden: »Von hier aus und eingebettet in ein Kollektiv entwerfen wir Fragen, die uns zu theoretischen Räumen führen. Hier bewegen wir uns suchend, hinterfragend, lernend. Hier werden Ansätze und Theorien weitergedacht, verarbeitet, verschränkt, entfaltet, in ein Verhältnis zur Erfahrung gebracht. Erkenntnisse ergeben sich. Manchmal. Perspektiven für politische Handlungen und Interventionen werden entworfen. Oder nicht. Andere Fragen entstehen. Immer wieder.«(Salgado 2014, 10)

\section{Literatur}

Afghanische Frauen in das kollektiv (2018). "Das Warten in Angst« - ein offener Brief Afghanischer Frauen zum Weltflüchtingstag, abrufbar unter: https://www.das-kollektiv.at/das-warten-angst-ein-offener-brief-afgha nischer-frauen-zum-weltfluechtingstag (letzter Zugriff: 9.6.2019).

Cusicanqui, Silvia Rivera (2019). Ch'ixinakax utxiwa: Eine Reflexion über dekolonisierende Praxen und Diskurse, in: COMPA/maiz/das kollektiv/ Entschieden gegen Rassimus (Hg.): Pädagogik im globalen postkolonialen Raum. Bildungspotenziale von Dekolonisierung und Emanzipation, Weinheim/Basel: Belz Juventa, 264-279. 
Cusicanqui, Silvia Rivera (2010). Orgullo de ser mestiza, Interview, in: Página 12, abrufbar unter: https:/www.pagina12.com.ar/diario/suplemen tos/las12/13-5889-2010-07-30.html (letzter Zugriff 25.5.2019).

Die zapatistischen Frauen (2019). Brief der Zapatistinnen an die Frauen, die auf der Welt kämpfen, abrufbar unter: http://enlacezapatista.ezln.org. $\mathrm{mx} / 2019 / 02 / 12 /$ brief-der-zapatistinnen-an-die-frauen-die-auf-der-weltkampfen/ (letzter Zugriff: 26.5.2019).

Garbe, Sebastian/Cárdenas, María/Sempertegui, Andrea (Hg.) (2018). Silvia Rivera Cusicanqui: Ch'ixinakax utxiwa. Eine Reflexion über Praktiken und Diskurse der Dekolonisierung. Aus dem Spanischen übersetzt von Silke Steiml und Emin Günaydin, Münster: Unrast.

Gohn, Maria da Glória Marcondes (2019). Retrospektive der brasilianischen Educação Popular und sozialer Bewegungen anhand der Konstruktion kollektiver Subjekte, in: COMPA/maiz/das kollektiv/Entschieden gegen Rassimus (Hg.): Pädagogik im globalen postkolonialen Raum. Bildungspotenziale von Dekolonisierung und Emanzipation, Weinheim/Basel: Belz Juventa, 154-170.

Kaloianov, Radostin (2017). Migrantische Kritik, in: Karasz, Lena (Hg.): Migration und die Macht der Forschung, Wien: ÖGB-Verlag, 137-162.

Lünenborg, Marreth/Fritsche, Katharina/Bach, Annika (2011). Migrantinnen in den Medien. Darstellung in der Presse und ihre Rezeption, Bielefeld: transcript. https://doi.org/10.14361/transcript.9783839417300.

Mauthausen Komitee Österreich (2017). Die FPÖ und der Rechtsextremismus. Lauter Einzelfälle?, abrufbar unter: https:/www.mkoe.at/sites/de fault/files/files/aktuelles/MKOE-Broschuere-Die-FPOE-und-der-Rechts extremismus.pdf (letzter Zugriff: 25.5.2019).

Salgado, Rubia (2014). Vor-Wort. Sprachliche Ermächtigung, in: maiz (Hg.): Deutsch als Zweitsprache. Ergebnisse und Perspektiven eines partizipativen Forschungsprozesses, 8-10, Linz: Eigenverlag, abrufbar unter: https://www.maiz.at/sites/default/files/images/deutsch-als-zweitspra che_www-2.pdf (letzter Zugriff 9.6.2019).

Schütze, Theresa (2016). (K)einen kühlen Kopf bewahren: Technokratie und Migrationsmanagement, abrufbar unter: http://www.migrazine.at/arti kel/keinen-k-hlen-kopf-bewahren-technokratie-und-migrationsmanagement (letzter Zugriff 25.5.2019).

Streck, Danilo R./Redin, Euclides/Zitkoski, Jaime José (2008) (Hg.). Dicionário Paulo Freire, Belo Horizonte: Autêntica Editora. 


\title{
Demokratie ist radikaler Experimentalismus
}

Rahel Süß

\begin{abstract}
Ohne Experimente gibt es keine Demokratie. In dem Beitrag wird ein experimenteller Zugriff auf das Thema Demokratie entwickelt und behauptet, dass liberale Demokratien auf Experimente angewiesen sind, um die Zukunft offenzuhalten. Ausgangspunkt bildet die Annahme, dass wir es gegenwärtig mit einem Zukunftsverlust zu tun haben, weil Demokratien die Möglichkeit einschränken, mit Alternativen zu experimentieren. Mein Lösungsvorschlag zielt auf eine handlungstheoretische Befragung des Ausgangspunktes moderner Demokratien und entwickelt das Experiment als demokratischen Schlüsselbegriff.
\end{abstract}

\section{Einleitung}

Zweifellos spricht einiges dafür, dass es um den Zustand liberaler Demokratien nicht gut bestellt ist (Brown 2015; Urbinati 2014). Die Einschätzungen zu den konkreten Defiziten sind vielfältig - sie reichen vom Anstieg nationalistischer Formen über die Unzufriedenheit mit den regierenden `Eliten und ihrer zeitlich an Wahlen orientierten Politik bis hin zur Kritik an institutionalisierten Formen neoliberaler Demokratie. Was allerdings ins Auge springt, ist ihr gemeinsamer Bezugspunkt, den ich in weiterer Folge als Zukunfts-Problem bezeichnen werde. Vor dem Hintergrund der Annahme, dass wir es heute mit einem Verlust der Zukunft zu tun haben, sind liberale (westliche) Demokratien daraufhin zu befragen, inwiefern sie die Möglichkeit von Veränderungshandeln - Experimente mit Alternativen - offenhalten oder einschränken. Die Behauptung ist, dass es ohne Experimente - soziale Praxen des Veränderungshandelns - keine wirkliche Demokratie gibt, weil erst über das Experiment die Zukunft in pluralen Gesellschaften offengehalten werden kann. Mein Vorgehen basiert auf einer dreifachen Aufgabe: 
Einen Erklärungsversuch der Frage, was das Experiment mit Demokratie zu tun hat, soll erstens eine Beschreibung des Zustands liberaler Demokratien liefern. Für die Behauptung, dass es sich beim Experiment um einen demokratischen Schlüsselbegriff handelt, werden zweitens Überlegungen dazu angeknüpft, was das Experiment eigentlich als demokratisch qualifiziert. Drittens gilt es zu erkunden, wie viel Experiment Demokratie braucht, um die Zukunft radikal offenzuhalten.

\section{Das Experiment als demokratischer Grundbegriff}

Zukunft ist als expliziter Bezugspunkt von gegenwärtigen Aushandlungsprozessen um Demokratie nicht wegzudenken. Allerdings haben wir es im Zusammenhang mit dem Zukunfts-Problem nicht mehr in erster Linie mit einer proklamierten Politik der Alternativlosigkeit zu tun, die über drei Jahrzehnte die theoretische und politische Diskussion prägte (Marchart 2013, 7 f.). Mittlerweile sind Alternativen zu neoliberaler Politik nicht nur vorstellbar geworden, sondern werden vehement von politischen Parteien und Bewegungen entlang des gesamten politischen Spektrums eingefordert. Während jedoch nationalistische und fremdenfeindliche Gruppierungen im Namen der Demokratie den Verlust einer nationalen Zukunft beklagen, um eine Pluralität an Freiheitszugewinnen wieder zurückzufahren, setzen Demokratiebewegungen auf eine Pluralität an Zukünften. Eine solche Diskrepanz zwischen den Verständnissen von Demokratie signalisiert nicht zuletzt den Bedarf, das Verhältnis von Demokratie und Zukunft neu zu denken, will man Demokratie als Bezugspunkt für Emanzipation nicht aufgeben.

\section{Was hat das Experiment mit Demokratie zu tun?}

Auf welcher Grundlage lässt sich nach Experimenten im Kontext von Demokratie fragen? Gewöhnlich ist die Rede vom Experiment an ein naturwissenschaftliches Verständnis von Versuch und Scheitern gebunden. Mal als Hypothese, um eine Idee zu testen. Mal als Modus der Übung, um etwas $\mathrm{zu}$ erproben oder $\mathrm{zu}$ erfinden. $\mathrm{Zu}$ experimentieren heißt danach $\mathrm{zu}$ fragen, wie sich die Gesetzmäßigkeiten eines Gegenstandes angesichts von spezifischen und wechselnden Bedingungen verhalten. Das Experiment, im hier 
vorgeschlagenen Sinne, ist aber auch ein politischer Begriff, weil es nach der Möglichkeit zu handeln fragt. In nichts kommt Experimentalität stärker zum Ausdruck als in der Frage "Kann ich - überhaupt - handeln?« (Menke 2013, 99). Das Experiment, so wie es von mir konzeptualisiert ist, versucht den Fehler zu vermeiden, das Gelingen einer Handlung mit der Möglichkeit zu handeln, sprich mit der Möglichkeit der Erprobung des Gelingens einer Handlung, zu verwechseln. Das Experiment als eine soziale Praxis des Veränderungshandelns zu verstehen heißt dann, angesichts der Ungewissheitserfahrung in demokratischen Gesellschaften von einer "provozierenden", also immer anwesenden Möglichkeit von Veränderungshandeln auszugehen. Gerade weil sich Möglichkeiten von Veränderungshandeln nicht aus gegebenen Umständen direkt ableiten oder im Vorhinein erschließen lassen, ist politisches Handeln auf das Experiment angewiesen.

$\mathrm{Zu}$ experimentieren bedeutet, sich seines Handelns und der Möglichkeit zu handeln zu versichern, ohne die Gewissheit, wie Erfahrungen ausgehen werden. Das Experiment ist auch deshalb eine soziale Praxis des Veränderungshandelns, weil das Experiment der Ort ist, von dem aus es möglich wird, Veränderung zu erproben und an den Erfahrungen zu testen. »Handeln im Modus des Experiments«, um es mit einem Ausdruck von Menke zu sagen, ist »ein Experiment mit der Möglichkeit des Handelns« (ebd.).

Mit einer solchen experimentellen Wendung lässt sich zeigen, dass zahlreiche heutige demokratische Versprechen auf eine offene Zukunft symptomatisch für einen Verlust von Zukunft stehen, weil sie die Möglichkeit von neuen Experimenten versperren, das heißt, die Möglichkeit für Veränderungshandeln untergraben und darüber strukturelle Ungleichheiten im $\mathrm{Zu}$ gang zu Demokratie stabilisieren. Anhand von zwei politisch aktuellen Beispielen möchte ich diese Behauptung untermauern.

Im ersten Fall bezeichnet Zukunft das neoliberale Versprechen auf individuelle Selbstverwirklichung. Charakteristisch für ein solches Versprechen ist die Vorstellung einer lebenslangen Erprobung und Ausarbeitung der eigenen Individualität. Passend zu diesem Verhaltensideal, das seit den 1970er Jahren als Legitimierungsgrundlage weitreichender gesellschaftlicher Umstrukturierungen dient, wurde der Spielraum für flexible Lebensentwürfe ausgeweitet. Was dabei nicht übersehen werden darf, ist, dass ein solches Versprechen auf Selbstverwirklichung ebenfalls die Möglichkeit von Veränderungshandeln untergräbt, indem zum Beispiel ein institutionell abgesicherter Individualismus soziale Gruppen spaltet und strukturelle Ungleich- 
heiten verdeckt. Sofern Subjekte angehalten, ermutigt oder durch repressive Maßnahmen dazu genötigt werden, »sich um ihrer Zukunftschancen willen zum Zentrum ihrer eigenen Lebensplanungen und Lebensführung zu machen« (Honneth 2010, 210), haben wir es mit einer Verdrängung von sozialen Problemen in den privaten Verantwortungsbereich zu tun, die die politische Handlungsmacht einschränkt.

Im zweiten Fall begegnen wir Zukunft als Versprechen auf digitalen Fortschritt und Freiheitszugewinne. Dass es sich auch bei diesem Versprechen eher um einen Verlust von Zukunft handelt, zeigt ein Blick auf zwei damit verbundene Schwierigkeiten, die die Möglichkeit von Veränderungshandeln betreffen: Einerseits werden Daten zum Werkstoff für Unternehmen und alle, die es sich leisten können, lassen ihre Daten schützen oder anonymisieren, um ihre Handlungsspielräume abzusichern. Andererseits wird die Möglichkeit von Veränderungshandeln blockiert, indem zum Beispiel die bei Gerichtsverfahren zum Einsatz kommenden Programme künstlicher Intelligenz strukturelle Diskriminierungen reproduzieren und stabilisieren (Pundy 2019). Gemäß dem Umstand, dass digitale Technologien zukünftiges Verhalten an den »Erfolgen « vergangener In- und Output-Koordinationen ausrichten (Wiener 1988, 33), sind sie nicht in der Lage, die Zukunft radikal offenzuhalten.

\section{Was qualifiziert das Experiment als demokratisch?}

Jedes Handeln ist ein Experiment. Es ist ein Experiment mit der Erfahrung, weil es die Möglichkeit des Gelingens von Handlungen erprobt. Aber folgt daraus unweigerlich, dass jedes demokratische Handeln ein Experiment ist? Führt eine solche Position nicht zu einer beispiellosen Unschärfe des Wortes selbst? Warum sollte man das Experiment überhaupt als demokratischen Grundbegriff behandeln? Für das Argument der Untersuchung - gemäß dem es sich beim Experiment um einen demokratischen Grundbegriff handelt - ist die Frage von Bedeutung, inwiefern die Idee des Experiments unser politisch-soziales Zusammenleben nachhaltig geprägt hat. Damit stoßen wir bereits auf eine erste Schwierigkeit, denn insofern das Experiment, anders als die weitaus geläufigeren Begriffe Freiheit, Gleichheit oder Solidarität, noch nicht Kernbestand unseres demokratischen Selbstverständnisses ist, können wir auch nicht nach einem feststehenden politischen Terminus suchen. Vor diesem Hintergrund erkunde ich, inwiefern Formen des 
Experiments das demokratische Selbstverständnis eines liberalen Kanons Freiheit der Person und Rechtsstaatlichkeit - geprägt haben, aber auch die Grenzen eines solchen engen Demokratieverständnisses zu sprengen beabsichtigen (Holthaus/Noetzel 2012; Ryan 2012).

Es ist an dieser Stelle kein Platz, um alle experimentellen Spielarten zu würdigen. ${ }^{1}$ Fürs Erste muss deshalb eine begriffliche Unterscheidung von drei Modellen des Experiments genügen. So lassen sich im Durchgang der verschiedenen Vorstellungen, von der Antike bis zur Gegenwart, folgende Kernvorstellungen des Experiments voneinander abheben: der hypothetische Gesellschaftsvertrag, die moralische Selbstgesetzgebung und die konfliktuelle Vergemeinschaftungsform. Als Ausgangspunkt kann die Beobachtung gelten, dass das Experiment ideengeschichtlich für das demokratische Selbstverständnis in jener Weise Bedeutung erhält, wie sich politische Ideen Schritt für Schritt von ihren Gewissheitsansprüchen lösen. Wollen wir das Experiment nämlich nicht so verstehen, dass es eine feststehende Wahrheit enthüllt, dann muss ihm die Möglichkeit der Veränderbarkeit als erstes demokratisches Grundprinzip eingeschrieben sein. Was auf dem Spiel steht, wenn dies nicht geschieht, zeigt sich beispielhaft am antiken Politikbegriff, dem mit seiner Anbindung an eine ideale politische Ordnung jegliche Offenheit hinsichtlich des historischen Verlaufs und der politischen Auseinandersetzung um die Gestaltung des sozialen Zusammenlebens abhandenkommt (Weber-Schäfer 2000).

Es sind besonders die Theorien des Gesellschaftsvertrages von Hobbes und Locke bis Rousseau, die im 17. und 18. Jahrhundert eine experimentelle Dimension im Kontext von demokratischen Fragestellungen zur Geltung bringen. Der Ausgangspunkt ihrer jeweiligen Konstruktion bildet der Grundsatz einer hypothetischen Frage: ${ }^{2}$ Wie muss die gesellschaftliche und staatliche Ordnung beschaffen sein, damit ihr freie Individuen zustimmen können? Entsprechend dem Gedankengang gilt eine bestimmte staatliche

1 Eine systematische Rekonstruktion des Experiments ist Teil meiner Dissertation, die den Titel trägt: Provozierte Demokratie - Eine radikale Demokratietheorie des Experiments.

2 John Rawls greift in seiner Theorie der Cerechtigkeit (1971) auf die experimentelle Argumentationsfigur der klassischen Gesellschaftstheorien zurück, um diese für das 20. Jahrhundert zu aktualisieren. Sein Versuch, von Vorstellungen des Guten unabhängige Gerechtigkeitsprinzipien zu identifizieren, basiert auf einem Schleier des Nichtwissens (veil of ignorance), der sich über den Vertragsabschluss legt. Dieser Schleier verhindert, dass die Individuen ihre Position in der Cesellschaft und den Zeitpunkt, zu dem sie leben, kennen. 
Ordnung nur dann als gerechtfertigt, wenn hypothetisch angenommen wird, dass ihr alle Individuen in einem vorgesellschaftlichen Zustand hätten zustimmen können. Aus der experimentellen Abstraktion des hypothetischen Vertragsabschlusses ergibt sich nun folgende Komplikation: Es wird der Blick darauf verstellt, dass die Mitglieder einer politischen Gemeinschaft als Urheber"innen und auch Erneuerer*innen ihrer eigenen Rechtsgrundsätze in Erscheinung treten können. Wenn wir unter Selbstregierung, wie im Fall des Gesellschaftsvertrages, nicht bloß den Umstand verstehen wollen, politische Macht zu begrenzen, um die Freiheit der Einzelnen abzusichern, dann muss das Experiment auch eine positive Form annehmen können, die es erlaubt, die Bedingungen für die Herstellung neuer Handlungszusammenhänge einzufangen. Wie kann das Experiment als demokratischer Grundbegriff die Möglichkeit berücksichtigen, dass Subjekte auf ihren eigenen Willen einwirken und ihre Handlungsziele selbst setzen? Eine Lösung für das Problem verspricht unser zweites Beispiel bereitzuhalten: das Experiment als moralische Selbstgesetzgebung.

Nicht anders als die Konstruktion eines hypothetischen Vertragsabschlusses führt uns auch die reflexive Form des Experiments als moralische Selbstgesetzgebung zu spezifischen Vorstellungen über die Legitimierung einer gesellschaftlichen Ordnung. Was allerdings bei den Vertragstheoretikern als Wille zur vertraglichen Einigung gilt, wird von Immanuel Kant in eine Vorstellung der unbedingten Vernunftnotwendigkeit eines moralischen Sittengesetzes überführt, das von der Prämisse einer reflexiven Selbstbeziehung des Subjekts ausgeht. Individuelle Freiheit erscheint dann als Ergebnis einer rationalen Selbstgesetzgebung, die sich an einem moralischen Prinzip der Verallgemeinerbarkeit orientiert. So müssen die Gründe, an die wir uns vernünftigerweise bei der Selbstbestimmung unseres Handelns halten können, in einem ersten Schritt als zumindest zustimmungsfähig vorgestellt werden können, um sie in einem zweiten Schritt auf ihre Verallgemeinerbarkeit zu prüfen. Individuelle Freiheit geht also mit Selbstbestimmung einher, sofern sie im kantischen Sinne mit einer moralischen Autonomie verknüpft ist. Diesem Verständnis nach sind wir nur in dem Maße frei, wie sich unser Handeln an verallgemeinerbaren Gründen orientiert. ${ }^{3}$

3 Eine Entsprechung findet diese Idee in Kants berühmter Gedankenfigur des kategorischen Imperativs: »Handle nur nach derjenigen Maxime, durch die du zugleich wollen kannst, dass sie ein allgemeines Cesetz werde.«(Kant 1990 ff., 421) 
Bei Jürgen Habermas (1983) findet die Vorstellung einer experimentellen Leistung, die wir bei Kant als transzendentale Präsupposition kennengelernt haben, eine empirische Aktualisierung. Bestand die experimentelle Leistung vormals in einer individuellen, von dem Subjekt auf sich selbst bezogenen Handlung, wird sie nun als Ergebnis eines gemeinsamen Handelns der Mitglieder einer Kommunikationsgemeinschaft aufgefasst. Kants Idee einer moralischen Autonomie - die Fähigkeit, das eigene Handeln mit selbstgesetzten Moralprinzipien zu verknüpfen - wird von Habermas als letzte Stufe eines Lernprozesses aufgefasst. Verstehen wir sein kommunikationstheoretisches Modell in dem Sinne, dass es die Möglichkeit von Veränderungshandeln berücksichtigt, so scheint das Experiment nicht mehr als ein bloßes Additiv von Freiheit, sondern als ihr Medium und ihre Vollzugsbedingung. Allerdings wollen beide, Kant als auch Habermas - und hier entsteht eine Komplikation -, Unparteilichkeit und Verallgemeinerungsfähigkeit als Handlungsrahmen verstanden wissen. Gemäß dieser Vorstellung kommt der besondere Vollzug von Freiheit darin zum Ausdruck, dass sich Individuen im Fall von Handlungskonflikten von allen existierenden sozialen Bindungen und Normen herauslösen und ihre Werte und Überzeugungen uminterpretieren und verflüssigen können (Habermas 1976, 80). Wollen wir aber nicht davon ausgehen, dass sich grundlegende Wertekonflikte in pluralen Gesellschaften miteinander versöhnen lassen, weil Konflikthaftigkeit und Pluralität konstitutiv für Demokratie sind, müssen wir nach einer Form des Experiments Ausschau halten, die eine konfliktuelle Vergemeinschaftung anerkennt.

Demokratien in pluralen Gesellschaften stehen vor der spannungsreichen Aufgabe, einen Umgang mit Wertkonflikten - Ansprüchen wie persönliche Autonomie und Gemeinschaft, Individualität und Solidarität - zu finden und gleichzeitig die Zukunft radikal für immer neue Konflikte offenzuhalten (Mouffe 2013; 2005). Denn weil Demokratie erst über offene Aushandlungsprozesse und soziale Beziehungen hergestellt wird - sei es institutionell oder auf der Ebene von Alltagspraxen im Kontext von Bildungs- oder Gesundheitseinrichtungen und in Bereichen wie Ernährung, Mobilität, Forschung oder Kunst -, ist sie auf das Experiment als konfliktuelle Vergemeinschaftung angewiesen. Eine solche Form des Experiments geht in einer entscheidenden Hinsicht über die Idee des hypothetischen Gesellschaftsvertrages und der moralischen Selbstgesetzgebung hinaus: Für die Behauptung, dass es sich beim Experiment um einen demokratischen Grundbegriff handelt, stellt diese Form des Experiments insofern eine 
Erweiterung des Denkhorizonts dar, als sie den Konflikt als konstitutiv für Demokratie anerkennt. Im Kern meint die Form des Experiments als konfliktuelle Vergemeinschaftung, dass über das Experiment gesellschaftliche Konflikte in pluralen Gesellschaften verhandelt werden. Solche experimentellen Aushandlungsprozesse - sei es im Rahmen von institutionellen Settings, periodischen Wahlen, alltäglichen Aushandlungsformen von Demokratie, selbstorganisierten Zusammenhängen, Formen des Protests und des Widerstandes - sind die Voraussetzung dafür, dass die Möglichkeit für Veränderungshandeln offengehalten wird und sich Gesellschaft immer wieder neu temporär gründen kann. Das heißt, dass bestimmte normative Vorstellungen von Freiheit, Gleichheit und Solidarität in einem politischen Projekt institutionell und im Alltagsverstand zeitweilig fixiert werden.

Meine definitorische Entscheidung besagt, vom Experiment nur dann zu sprechen, wenn die soziale Praxis des Veränderungshandelns an soziale Konflikte angebunden ist. Da politische Praxen also Versuche darstellen, Konflikte zu verhandeln, handelt es sich bei ihnen um Experimente. Verhandlungen von Konflikten sind stets experimentierend, weil Lösungen für Konflikte weder aus gegebenen Umständen direkt ableitbar noch im Vorhinein erschließbar sind. Konfliktverhandlungen sind vielmehr auf ein dynamisches Probehandeln - ein Ausprobieren im Modus von Versuch und Scheitern - angewiesen. Für den Fall, dass die Beziehung von politischen Subjekten zu sich selbst, den anderen und der Umwelt Ausdruck eines praktischen Eingelassenseins in eine niemals vollständig verfügbare Identität, Andersheit oder Welt ist, meint das Experimentelle eine bestimmte Art und Weise, sich in Beziehung zu setzen und sich die sozialen Verhältnisse experimentell aneignen zu können. Denn indem eine solche Form des Selbst- und Weltbezugs nicht bloß der Anwendung universeller Prinzipien folgt, müssen die normativen Bezugspunkte von Handlungen im Rahmen von Konflikten - alltäglichen Aushandlungen von Demokratie - erprobt, geprüft und hergestellt werden. In diesem Licht erscheinen Konflikte dann als Testgelände von normativen Standards, politischen Selbstverständnissen und demokratischen Erfahrungen, um jene temporär über das Experiment zu verhandeln und zu fixieren.

Anders als der naturwissenschaftliche Experimentbegriff, dessen kognitive Ausrichtung auf die Organisation von Erfahrung und die Freilegung von Erkenntnis - Gesetzmäßigkeiten eines Gegenstandes - zielt, befasst sich mein demokratischer Experimentbegriff mit der Möglichkeit des Handelns selbst. Zu experimentieren heißt, dass wir handeln, um eine Erfahrung 
mit der Erfahrung zu machen. Konfliktprovokationen entsprechen in diesem Sinne Handlungsexperimenten, in denen Subjekte die Erfahrung machen können, dass Veränderung von ihnen selbst ausgehen kann, auch wenn es sich dabei zunächst nur um eine Erprobung im Sinne einer Provokation handelt, ohne die Gewissheit auf einen erfolgreichen Ausgang von Experimenten.

Damit in pluralen Demokratien die Möglichkeit von Veränderungshandeln offengehalten werden kann, müssen Konflikte beständig neu von den Teilnehmer*innen einer politischen Gemeinschaft provoziert werden. Es sind die Resultate von Konfliktverhandlungen daraufhin zu befragen, ob sie die Anbindung an und die Konfrontation mit weiteren Konflikten ermöglichen oder behindern. $\mathrm{Zu}$ den Artikulationsformen von Konfliktprovokation zählen politische Wahlen ebenso wie alltägliche Aushandlungen von Demokratie mit ihrer Verteidigung oder Aufgabe persönlicher Freiheiten, dem $\mathrm{Zu}$ lassen von oder Aufbegehren gegen Ungerechtigkeit. Es zählen dazu aber auch Formen des Widerstandes und der demokratischen Selbstorganisation sowie Handlungen der Solidarität und des Sich-Einsetzens für andere.

\section{Wie viel Experiment braucht Demokratie?}

Mit den Ausführungen zum demokratischen Experiment sind wir nun ausreichend gerüstet, um zur Ausgangsthese zurückzukehren. Was würde es bedeuten, die Fähigkeit experimentell zu handeln - das heißt Konflikte zu provozieren - als ein demokratisches Merkmal von pluralen Gesellschaften $\mathrm{zu}$ verstehen? Es sind mindestens zwei Gründe, die dafürsprechen, das Experiment als konstitutiv für Demokratie anzuerkennen. Wir haben gesehen, dass es sich beim Experiment nicht bloß um ein Merkmal von Demokratie handelt, sondern, dass keine demokratischen Praxen, Institutionen und Selbstverständnisse außerhalb einer experimentellen Dimension bestehen können, sofern sie die Möglichkeit von Veränderungshandeln offenhalten wollen. Erst indem Konflikte provoziert werden, die dann als Testgelände dienen, kann Demokratie ihr Versprechen auf eine offene Zukunft und die Möglichkeit von Veränderungshandeln einlösen. Schließlich kann über Experimente ein Mehr an Demokratie, also ein Mehr einer Praxis der Konfliktprovokation, die Möglichkeit von Veränderungshandeln nicht nur offenhalten, sondern gesellschaftliche Transformationen auch anstacheln. Indem nicht nur gesellschaftliche Schließungen infrage gestellt, sondern alternative 
Handlungsmöglichkeiten in einem experimentellen Sinn provoziert und organisiert werden, lassen sich demokratische Forderungen auf immer neue soziale Sphären ausweiten. Demokratie als radikaler Experimentalismus meint dann eine Form der Radikalisierung durch Demokratie, im Sinne einer Pluralisierung von Gleichheitseffekten, die die Möglichkeiten von demokratischem Veränderungshandeln auf soziale Sphären und Beziehungen ausweitet.

\section{Demokratietheorie als Theorie des Experiments}

Vor diesem Hintergrund ist eine Antwort auf die Ausgangsfrage der Untersuchung - was es mit dem Experiment als demokratischem Grundbegriff auf sich hat - gewonnen. Das Ziel der Untersuchung war es, einen experimentellen Zugriff auf das Thema Demokratie zu entwickeln, der es erlaubt, das ausgewiesene Zukunfts-Problem von liberalen Demokratien umfassender in den Blick zu bekommen. Die von mir entwickelte Alternative lief auf folgenden Befund hinaus:

Demokratie ist, will sie die Möglichkeit von Veränderungshandeln offenhalten, auf das Experiment angewiesen. Denn erst über das Experiment können Konflikte in pluralen Gesellschaften verhandelt und demokratische Ordnungen temporär neu gegründet werden. Pointiert formuliert ist das Experiment beides: bedrohend provozierend und demokratisch. Im ersten Fall verweist das Experiment, angesichts der Ungewissheitserfahrung in demokratischen Gesellschaften, auf eine provozierende, das heißt immer anwesende Möglichkeit von Veränderungshandeln. Das Experiment ist aber auch demokratisch, weil es die Möglichkeit von Offenheit und Gleichheit an das Versprechen bindet, dass die Vielen herrschen können, auch wenn sie damit scheitern. Demokratietheorie muss demzufolge, will sie praktisch anschlussfähig sein, als Theorie des Experiments aktualisiert werden, so mein Fazit.

\section{Literatur}

Brown, Wendy (2015). Undoing the demos: Neoliberalism's stealth revolution. Cambridge: MIT Press.

Habermas, Jürgen (1976). Moralentwicklung und Ich-Identität, Frankfurt a. M.: Suhrkamp. 
Habermas, Jürgen (1983). Moralbewusstsein und kommunikatives Handeln, Frankfurt a. M.: Suhrkamp.

Holthaus, Leonie/Noetzel, Thomas (2012). Demokratischer Pluralismus versus despotische Herrschaft, in: Lembcke, Oliver/Ritzi, Claudia/Schaal, Gary (Hg.): Zeitgenössische Demokratietheorie. Band 1: Normative Demokratietheorien, Wiesbaden: Springer VS, 33-62. https://doi.org/10.1007/9783-531-94161-5_2.

Honneth, Axel (2010). Das Ich im Wir. Studien zur Anerkennungstheorie, Frankfurt a. M.: Suhrkamp.

Kant, Immanuel (1990 ff.). Grundlegung zur Metaphysik der Sitten, Bd. IV Immanuel Kants Gesammelte Schriften, Berlin: Georg Reimer.

Marchart, Oliver (2013). Das unmögliche Objekt. Eine postfundamentalistische Theorie der Gesellschaft, Frankfurt a. M.: Suhrkamp.

Menke, Christoph (2013). Die Kraft der Kunst, Frankfurt a. M.: Suhrkamp.

Mouffe, Chantal (2005). The Democratic Paradox, London/New York: Verso.

Mouffe, Chantal (2013). Agonistics. Thinking the world politically, London/ New York: Verso.

Pundy, Doris (2019). Wenn der Richter mit Softwarehilfe urteilt, abrufbar unter: https://p.dw.com/p/3BwGJ (letzter Zugriff 15.7.2019).

Rawls, John (1979) [1971]. Eine Theorie der Gerechtigkeit, Frankfurt a. M.: Suhrkamp.

Ryan, Alan (2012). Liberalism, in: Goodin, Robert/Pettit, Philip/Pogge, Thomas (Hg.): A Companion to Contemporary Political Philosophy, Malden: Wiley-Blackwell, 360-282.

Urbinati, Nadia (2014). Democracy disfigured, Cambridge: Harvard University Press. https://doi.org/10.4159/harvard.9780674726383.

Wiener, Norbert (1988). The Human Use of Human Beings: Cybernetics and Society. Cambridge: Dacapo Press.

Weber-Schäfer, Peter (2000). Der Politikbegriff der Antike, in: Lietzmann, Hans/Nitschke, Peter (Hg.): Klassische Politik. Politikverständnisse von der Antike bis ins 19. Jahrhundert, Wiesbaden: VS Verlag für Sozialwissenschaften, 11-21. https://doi.org/10.1007/978-3-322-93221-1_1. 



\title{
Eine Demokratie lernen, die es noch nicht gibt
}

\author{
Nora Sternfeld
}

\begin{abstract}
Wie können wir eine Demokratie lernen, die es noch nicht gibt? Einerseits klingt es paradox - wird Lernen doch als Herrschaftstechnik zur nachträglichen Praxis, was sie überprüfbar und berechenbar macht. Andererseits ist Lernen doch eigentlich gerade das: Vorweglernen. Wenn Antonio Gramsci davon ausgeht, dass jedes Verhältnis von Hegemonie ein pädagogisches Verhältnis ist und als solches verändert werden kann, dann können wir eben diese Machtverhältnisse verlernen und eine Welt erforschen, die es noch nicht gibt. Anhand einer Auseinandersetzung mit der Idee der Gleichheit bei Rancière sowie einer Reflexion des Projekts Making Democracy gehe ich dieser Frage nach.
\end{abstract}

»Wenn du lernst, kämpfst du dagegen.«Zitat einer Teilnehmerin und Lernenden im Rahmen von maiz ${ }^{1}$

"We were the folks who knew that whether we were in an academic setting or not, we would continue to study, to learn, to educate.«-bell hooks $(2003,49)$

Stell dir vor, wir wären alle gleich und würden nur durch die Verhältnisse ungleich gemacht. Durch Verhältnisse, die alles daransetzen, Unterscheidungen $\mathrm{zu}$ machen. Mit immer mehr Berechnungsmaschinen und Datennetzwerken, sodass es in der Wissensökonomie, $\mathrm{zu}$ deren Industrie das Lernen heute gehört, nicht einmal möglich scheint, dass manche sich in der

1 Siehe den Beitrag von das kollektiv in diesem Band. 
Ungleichheit gleich finden. Lernprozesse (mit ihren Aufnahmeprüfungen, Deutschkursen, Intelligenztests, Noten, Durchschnittsermittlungen, Fragebögen und Selbstevaluierungen) ordnen uns zunehmend gegeneinander an und sind somit ein wesentlicher Mechanismus der Herstellung von Ungleichheit. ${ }^{2}$ Aber, so der Ausgangspunkt des Projekts Making Democracy, sie sind auch Kontexte, in denen trotz alledem ein Insistieren auf Gleichheit, Freiheit und Solidarität stattfinden kann. Wo wir zusammenkommen und miteinander lernen, manifestieren sich zwar Machtverhältnisse, aber es werden darüber hinaus Momente der Ungleichheit angesprochen. So können Bildungsprozesse nicht nur Wege aus der Ungleichheit, Unfreiheit und Vereinzelung sein, weil sie Mittel an die Hand geben, diese infrage zu stellen, sondern weil sie Räume sind, um andere Formen des Zusammenseins und des Handelns zu erproben. In meinem Beitrag möchte ich zunächst herausfinden, was es heute heißt, sich Gleichheit vorzustellen, um Ungleichheit $\mathrm{zu}$ adressieren. Dass dies, so schön es klingt, im Konkreten konfliktuell ist, thematisiere ich anschließend anhand von Aspekten des Projekts Making Democracy.

\section{Stell dir also vor, wir wären alle gleich}

Eine solche Imagination formulierte Jacques Rancière (2007) mit einem Rückgriff auf Jacotot, einen französischen Lehrer und Erziehungstheoretiker des ausgehenden 18. und beginnenden 19. Jahrhunderts, in seinem Buch Der unwissende Lehrmeister. Die Imagination scheint unter den Bedingungen allgegenwärtiger Eignungsprüfungen, Selbsttests und Kompetenzorientierungen geradezu abwegig - und ist vielleicht gerade deshalb ein Wagnis wert. Denn zumeist sind Lernkontexte keineswegs emanzipatorisch. Sie sind Trainingsräume der Anpassung: Anpassung an die Institutionen und die Vergesellschaftungsformen der Gegenwart. Dies geschieht mit Mitteln des Zwangs, des Drucks und des Sogs, der unsere Lust zu lernen zum Einsatz bringt, und es betrifft alle am Bildungsprozess Beteiligten. Dabei werden

2 »Seit Pisa ein Test ist, führen hierzulande die schlechten Ergebnisse im internationalen Vergleich nicht zur Reform der überkommenen Bildungssysteme, sondern sie heizen vor allem die allseitige Hetze gegen jene Schüler an, deren Eltern oder Großeltern aus dem Ausland stammen.«(Rabinovici 2019, 24) 
wirkmächtige Unterscheidungen vorangetrieben, indem mit schulischen Mitteln die Besseren von den Schlechteren, in alltäglichen Auseinandersetzungen die Cooleren von den Uncooleren, die Fähigen von den Unfähigen differenziert werden. Und es werden Mittel der gegenseitigen Berechnung und Überwachung (der Evaluierung, Beschreibung, Benotung und des Feedbacks) eingesetzt. Die Unterscheidungen korrelieren mit gesellschaftlichen Strukturen und Marginalisierungen - es spielen selbstverständlich auch Biografien und psychologische Aspekte mit hinein. Manchmal tritt die Übereinstimmung deutlich zutage, manchmal merkt man sie kaum, oder nur, wenn sie einen selbst betrifft.

In einer Zeit, in der Pädagogik also wesentlich im Hinblick auf zählbare Unterscheidungen ${ }^{3}$ organisiert und strukturiert ist, in der Demokratie zur Kompetenz wird, in der Potenzialentfaltung messbar und kulturelle Bildung zunehmend identitär wird ${ }^{4}$, möchte ich die Idee einer Gleichheit des Lernen-Könnens, die wir immer schon gemeinsam haben, neu verstehen und aktualisieren. ${ }^{5}$ Was wäre, wenn wir davon ausgingen, dass alle Menschen die gleiche Intelligenz besitzen (Rancière) ${ }^{6}$, dass alle Menschen Künstler*innen (Beuys), alle Philosoph*innen (Gramsci 1994, 1387) und alle Forscher*innen (Appadurai 2006, 167) ${ }^{7}$ sind? In gewisser Weise haben wir uns, wenn wir

3 Denken wir an Kompetenzmodelle, Wirkungsindikatoren und Leistungstransparenz, die letztlich alle auf Zählbarkeit abzielen und uns so im Verhältnis zu einander anordenbar machen (vgl. Herzig/Martin 2017).

4 Im Begriff der kulturellen Bildung liegt einerseits ein Versprechen, er hat andererseits eine Abgrenzungsfunktion. Nicht selten wird damit eine Auseinandersetzung mit »anderen Kulturen « bezeichnet (vgl. Mörsch 2016). Heute werden in Förderprogrammen, die die kulturelle Bildung adressieren, nicht selten folkloristische und heimatkundliche Projekte unterstützt. Den »anderen Kulturen«steht dann die »eigene Kultur« gegenüber, als gäbe es diese in Reinform.

5 Danke für das Insistieren auf diesen Gedanken an Hannes Glück (Promotionsprojekt Das geteilte Sinnliche. Politik und Poetik der Emanzipation bei Jacques Rancière).

6 Weiterführend zu diesem Gedanken vgl. Sternfeld 2009, 27-34.

7 »Research is normally seen as a high-end, technical activity, available by training and class background to specialists in education, the sciences and related professional fields. It is rarely seen as a capacity with democratic potential, much less as belonging to the family of rights. In this paper, I will argue that it is worth regarding research as a right, albeit of a special kind. This argument requires us to recognise that research is a specialised name for a generalised capacity, the capacity to make disciplined inquiries into those things we need to know, but do not know yet. All human beings are, in this sense, researchers, since all 
zusammen lernen, darauf geeinigt, dass wir etwas gemeinsam haben, dass es etwas gibt, das wir alle können, ein Vermögen, das uns alle zu denen gemacht hat, die wir sind: nämlich zu lernen und uns dabei zu verändern. ${ }^{8}$ Was könnte es also heißen, davon auszugehen, dass alle etwas verstehen und sich auf etwas verstehen können - dass wir gleich sind, weil wir lernen können -, ohne dabei gleich gemacht zu werden? Denn, wenn wir nicht erst evaluieren und selektieren müssten, wenn wir nicht immer schon in einem mathematischen System gegen einander anordenbar gemacht worden wären, bevor wir voneinander lernen, könnten sich Unterschiede vor einem anderen Hintergrund manifestieren. Wir wären auf einer gemeinsamen Basis unterschiedlich, bei der nicht jedem Lernprozess bereits ein Bewerten und Berechnen, Aussieben und Anordnen vorausgehen würde. Aber wir finden uns eben nicht unterschiedlich, sondern immer wieder bereits unterschieden wieder (vgl. Becker-Schmidt 1998). Wir werden innerhalb der real existierenden Machtverhältnisse ungleich gemacht.

Nehmen wir das Beispiel der Kunsthochschulen und ihrer Produktion sozialer Unterscheidungen. Cathrin Seefranz und Philippe Saner widmeten sich in einer Studie über Schweizer Kunsthochschulen den spezifischen Ein- und Ausschlüssen, die durch ein wie sie schreiben "preserve of the priviledged « produziert und perpetuiert, also hergestellt und erhalten werden (vgl. Seefranz/Saner 2013). Gerade gegen jene dort analysierten rassistischen und klassistischen Strukturen der Kunsthochschule stellt die *foundationClass der Kunsthochschule Weißensee in Berlin eine Intervention in gesellschaftliche und institutionelle Ausschlüsse dar:

»Die *foundationClass unterstützt nach Deutschland migrierte und/oder geflohene Menschen dabei, gleichberechtigten Zugang zu Kunsthochschulen zu erhalten. Sie schafft einen sozialen Raum, in dem die Ressourcen jeder einzelnen Person anerkannt und gewertschätzt und neue Formate des Kompetenzaustauschs und der Wissensvermittlung entwickelt werden. Erklärtes Ziel der *foundationClass ist es darüber hinaus, die Normen, Konventionen

human beings make decisions that require them to make systematic forays beyond their current knowledge horizons. (Appadurai 2006, 167)

8 »Die Cleichheit der Intelligenzen ist das einigende Band des Menschengeschlechts, die notwendige und zureichende Bedingung dafür, dass eine Gesellschaft von Menschen existiert.«(Rancière 2007, 90) 
und den Habitus von Kunstinstitutionen sowie die Kunstindustrie als Ganzes in Frage zu stellen. ${ }^{9}$

Ähnlich operierte der Lehrgang Mezzanin von maiz von März 2013 bis August 2014. Unter dem Titel »sich einmischen. different possibilities of self-representation « entstanden Animations-, Video- und Filmarbeiten von jugendlichen Migrant*innen in laborähnlichen Workshops, die die Basis für die Aufnahmeprüfung an der Akademie der bildenden Künste Wien werden sollten:

»Cemeinsam mit der künstlerischen Begleiterin Marissa Lôbo, tauschten sich die Jugendlichen über ihre Realität aus und wagten es, Entwürfe über ihre Bildungs- und Berufszukunft zu entwickeln. Themen wie Anwesendsein und Nicht-Hier-Sein-Dürfen, Fressen und Gefressen-Werden, Notorisch-Cefragt- und Interviewt-Werden und Keine-Antworten-Bekommen, sexuelle Identität und Homophobie, verborgene eigene Geschichten, Schwarzsein und ästhetischer Widerstand, Überschreitung der Crenzen und Faktum der Grenzen waren ihre Ausgangspunkte. $\ll^{10}$

Die Aufnahmeprüfung durch Vorbereitung ganz legal zu unterwandern ist ein Eingriff in die Behauptung der Ungleichheit der Begabung: Erstens intervenieren die Projekte in die rassistischen und klassenreproduzierenden Strukturen der Kunst, in der jene, die entscheiden, wer hinein soll und wer nicht, der Gefahr nicht entkommen, sich selbst zu klonen. Zweitens intervenieren sie in den Glauben an die Selektion und Einteilbarkeit der Bewerber*innen - die zumeist nichts anderes misst als das Maß an Vertrautheit mit den selbstreferentiellen und hochdistinktiven Diskursen und Formensprachen der Gegenwartskunst. Dies scheint der eigentliche Grund zu sein, warum Projekte wie jene von maiz und der *foundationClass überhaupt wirksam werden können. Denn weil es keine »objektiven« Kriterien für Begabung gibt und letztlich nur Vertrautheit mit Techniken und Diskursen abgefragt werden kann, unterwandern und entlarven diese Projekte die ungleichmachende Praxis der Aufnahmeprüfung. Gegen die Behauptung der Ungleichheit wird hier gemeinsam an der Ermöglichung der Aufnahme in die Kunsthochschule gearbeitet - als potenzielles Fenster in eine andere $\mathrm{Zu}$ -

9 http://www.foundationclass.org/about/ (letzter Zugriff: 22.6.2019). 10 http://www.herein.at/home.php?il=15 (letzter Zugriff: 22.6.2019). 
kunft. Ganz in diesem Sinne heißt es im Programm der "foundationClass der Kunsthochschule Weißensee: »Together we want to find answers to the question of how the art schools of the future would look if they really recognised migration as an essential societal factor. $\aleph^{11}$ Die Projekte sind also zugleich Lernort und Praxis der Imagination einer Kunsthochschule, die einer Migrationsgesellschaft adäquat wäre - der Migrationsgesellschaft, in der wir doch längst sind.

Gleichheit als Heterogenität ist somit Ausgangspunkt und zugleich Forderung der Interventionen - sie ist ebenso deskriptiv wie präskriptiv. Dass wir in aller Unterschiedlichkeit zusammen lernen und zusammen arbeiten, ist selbstverständliche Realität und zugleich scheinbar unerreichbare Utopie in einer Gesellschaft, die teilt und trennt, die offensichtlich ihrer eigenen Heterogenität nicht gerecht wird. Wir haben es mit Institutionen zu tun, die Unterschiedlichkeit messen und mathematisieren, statt sie auszuhalten und damit gesellschaftliche Ungleichheiten verstärken oder erst schaffen. Eine Gleichheit des Lernens zu leben und vorstellbar zu machen bedeutet, mit einer Intervention in die reale Ungleichheit zu beginnen. So schlage ich vor, mit Jacques Rancière davon auszugehen, dass wir eben gleicher sind, als wir gemacht werden, und mit Antonio Gramsci wiederum davon, dass jedes Verhältnis von Hegemonie ein pädagogisches Verhältnis ist. ${ }^{12}$ Damit meint Gramsci, dass Macht immer Einverständnis braucht, sogar auf Einverständnis basiert, also gelernt wird - und gerade deshalb verändert werden kann. Wenn wir dies annehmen, folgt daraus, dass wir diese Machtverhältnisse, die uns ungleich machen, eben verlernen und eine Welt erforschen können, die es noch nicht gibt. Eine radikaldemokratische Demokratisierung der Demokratie ${ }^{13}$ heißt, auf der Bildungsebene zu klären, dass es in ungleichen Verhältnissen eine fast unvorstellbare und doch notwendige Aufgabe ist, Gleichheit zu denken, zu fordern, sich vorzustellen und kontrafaktisch zu erproben.

11 Auszug aus dem Programm der "foundationClass, Kunsthochschule Weissensee, abrufbar unter: http://www.foundationclass.org/programme/ (letzter Zugriff: 22.6.2019).

12 »]edes Verhältnis von Hegemonie ist notwendigerweise ein pädagogisches Verhältnis.« (Gramsci 1994, 1335)

13 Ich beziehe mich auf das Denken des politischen Theoretikers Oliver Marchart, dessen Buch Der demokratische Horizont ausgehend von Laclau und Mouffe nach einer Demokratisierung der Demokratie fragt, also nach einer Demokratie, die freier, gleicher und solidarischer ist als die, in der wir leben (Marchart 2019, im Erscheinen). 


\section{Wie würden wir dann Demokratie machen?}

Making Democracy ging von einer Reflexion der oben angesprochenen Unterscheidungen und zugleich von der nichtsdestotrotz alltäglich gelebten Reziprozität - also von der Tatsache, dass wir dennoch in geteilten Räumen, wie etwa der Schule, aufeinandertreffen und miteinander handeln und aufeinander bezogen sind - als Basis einer Demokratisierung aus. Davon, dass wir ständig lernen, uns ständig etwas beibringen ${ }^{14}$ und auch davon, dass es im Alltag ständig Rebellion gibt. Es ging darum, eine Praxis des Forschens und Lernens zu entwickeln, die das Potenzial einer Demokratisierung der Demokratie im Alltag verortet. Dabei sollte mitten im Herrschaftsraum Schule ein Experimentierfeld für die Erfahrung, Erprobung und Reflexion eigener Aushandlungsprozesse von Freiheit, Gleichheit und Solidarität hergestellt werden. ${ }^{15}$ Die zentrale Frage, was wir für eine demokratischere Demokratie aus Alltagspraxen in der real existierenden Demokratie voneinander lernen können, verlangte allen Beteiligten komplexe Denkprozesse ab: Zuerst einmal eine Auseinandersetzung damit, dass wir nicht in der demokratischsten aller Demokratien leben. Außerdem die Erfahrung, dass wir das eigentlich wissen, manchmal resignierend damit leben, manchmal damit hadern und uns nicht selten auf unterschiedliche Weisen dagegen wehren.

Der Widerstand, der, wie Foucault $(2004,282)$ schreibt, überall da ist, wo Macht ist, ist in der Schule oft passiv, bleibt vage und implizit. Im Zuge des Projektprozesses wurde er - vor allem im zweiten Jahr ${ }^{16}$ - explizit. Er richtete sich zunächst gegen die Widersprüche im Projekt selbst: gegen die oft leeren Versprechungen einer Emanzipation in der Projektlogik, gegen die scheinbare Freiheit, Gleichheit und Solidarität, die natürlich in der real existierenden Schule nicht verwirklicht werden können und schon gar nicht im Rahmen eines Projekts, das sichtbare und zum Teil wiederum quantifizierbare Ergebnisse zeitigen soll. So traten also - ganz im Sinne eines gemein-

14 bell hooks schreibt: »Embracing the concept of a democratic education we see teaching and learning as taking place constantly.« (hooks 2003, 41)

15 Rahel Süß spricht von Experimenten als wesentlichen Bestandteil von Demokratie: Sie versteht »das Experiment als konfliktuelle Vergemeinschaftung « und sagt, dass es ohne Experimente keine Demokratie geben kann (siehe den Beitrag von Rahel Süß in diesem Band).

16 Siehe die Beiträge von Elke Rajal und Nora Landkammer in diesem Band. 
samen Aushandelns von Demokratie - Widersprüche zutage. Doch was ist, wenn Lernende mit einem Projekt, das davon handelt, nicht einverstanden zu sein, nicht einverstanden sind? Ein Lernprozess für alle Beteiligten wird in Gang gesetzt: Das Projekt führte zu Protestformen der Schüler*innen die sich gegen schulische Abläufe und Zwänge sowie manchmal gegen das Projekt richteten - und machte Frustrationen und Widersprüche explizit, die sonst unausgesprochen bleiben. Besonders interessant war, so das Projektteam ${ }^{17}$, die Manifestierung des frustrierenden Widerspruchs zwischen Versprechen und Realität, der neoliberale Institutionen kennzeichnet. ${ }^{18} \mathrm{Ju}$ gendliche wehren sich, denn sie sind ständig - in der Werbung, in den Institutionen und auch zu Hause - mit Erwachsenen konfrontiert, die ihnen eine Welt vorspielen, die es nicht gibt. ${ }^{19}$ Kritik kann heißen, dies zu entlarven, es nicht zu akzeptieren.

Vielleicht liegt in der Weigerung, sich auf die leeren Versprechen einzulassen, ein wesentlicher Moment der Politisierung. Der deskriptiv-präskriptive Ausgangspunkt des Projekts musste also ausgehandelt werden. Und worin liegt der Unterschied zwischen der Zukunft als leerem Versprechen in der Postdemokratie und einem real-utopischen radikaldemokratischen Experiment? Die Demokratie wird nicht demokratischer, indem sie bloß behauptet wird. Wollen wir die Demokratie demokratisieren, müssen wir uns eingestehen, dass diese längst nicht so demokratisch ist, wie sie sich darstellt. ${ }^{20}$ Wie ich zu zeigen versuchte, liegt die Behauptung der Gleichheit eben nicht in ihrer bloßen Behauptung, sondern vielmehr in der Konfrontation mit der realen Ungleichheit und die Radikalisierung der Demokratie in der Auseinandersetzung mit ihrer Entleerung. Sehr anschaulich beschreibt Nora Landkammer in ihrem Beitrag in diesem Band die Entrüstung der Schüler*innen im Moment der Begegnung mit postdemokratischen Leer-

17 Siehe den Beitrag von Nora Landkammer in diesem Band.

18 Post-Politik und Real-Utopie haben tatsächlich eine gewisse spekulative Dimension gemeinsam - beide behaupten im Hinblick auf die Zukunft etwas, das es nicht gibt, aber geben könnte.

19 Dank an die im Projekt Making Democracy maßgeblich beteiligte Lehrende Beate Wallner für diese Überlegung.

20 Gergana Mineva und Rubia Salgado von das kollektiv schreiben in ihrem Beitrag in diesem Band im Zusammenhang mit der »unvollständigen Staatsbürgerschaft zweiter Klasse« von einer »trügerischen Inklusion«, von »trügerischen Bürgerrechten«. 
formeln auf der Website DemokratieWEBstatt ${ }^{21}$, einem Angebot des österreichischen Parlaments zur politischen Bildung. Diese Entrüstung richtete sich auch gegen die Widersprüche des Projekts - dessen real-utopische Dimension nun auf dem Prüfstand war. Und so kam es zu einem konfliktuellen Aushandlungsprozess, bei dem experimentell gemeinsam und voneinander gelernt wurde, was keineswegs leicht war. Denn eine »Sprengung in nächster Nähe«, wie die Lehrerin Beate Wallner es auf der Tagung des Projekts ausdrückte, »ist höchst unangenehm. « Doch gerade diese Erfahrung fordert die Entdemokratisierung, die Ungleichheit heraus. Sie realisiert Gleichheit. Dass dies nicht nur schön ist, ist eine Erfahrung mitten in der Demokratie.

\section{Literatur}

Appadurai, Arjun (2006). The right to research, in: Globalisation, Societies and Education, Vol. 4(2), https://doi.org/10.1080/14767720600750696.

Becker-Schmidt, Regina (1998). Trennung. Verknüpfung. Vermittlung. Zum feministischen Umgang mit Dichotomien, in: Knapp-Axeli, Gudrun (Hg.): Kurskorrekturen. Feminismus zwischen kritischer Theorie und Postmoderne, Frankfurt a. M./New York: Campus.

Foucault, Michel (2004). Geschichte der Gouvernementalität I. Sicherheit, Territorium, Bevölkerung, Frankfurt a. M.: Suhrkamp.

Gramsci, Antonio (1994). Gefängnishefte. Kritische Gesamtausgabe, Band 6, Hamburg: Argument.

Herzig, Bardo/Martin, Alexander (2017). Erfassung und Messbarkeit von Medienkompetenz als wichtige Voraussetzung für politische Bildung, abrufbar unter: https://www.bpb.de/lernen/digitale-bildung/medienpae dagogik/medienkompetenz-schriftenreihe/257604/erfassung-und-mess barkeit-von-medienkompetenz (letzter Zugriff: 27.6.2019)

hooks, bell (2003). Teaching Community, New York: Routledge.

Marchart, Oliver (2019). Der demokratische Horizont. Politik und Ethik radikaler Demokratie, Berlin: Suhrkamp (im Erscheinen).

21 https://www.demokratiewebstatt.at/thema/schule-und-demokratie/ (letzter Zugriff: 8.7.2019). 
Mörsch, Carmen (2016). Urteilen Sie selbst: Vom Öffnen und Schließen von Welten, abrufbar unter: https://www.kultur-oeffnet-welten.de/positionen/ position_2944.html (letzter Zugriff: 27.6.2019).

Rabinovici, Doron (2019). I wie Rabinovici. Zu Sprachen finden, Wien: Sonderzahl.

Rancière, Jacques (2007). Der unwissende Lehrmeister. Fünf Lektionen über die intellektuelle Emanzipation, Wien: Passagen.

Seefranz, Catrin/Saner, Philippe (2012). Making Differences: Schweizer Kunsthochschulen. Explorative Vorstudie, abrufbar unter: https://blog.zhdk.ch/ artschooldifferences/files/2013/11/Making_Differences_Vorstudie_End version.pdf (letzter Zugriff: 16.6.2019).

Sternfeld, Nora (2009). Das pädagogische Unverhältnis, Wien: turia + kant.

trafo.K (2014). Handbuch. Arbeiten im Kulturbereich, abrufbar unter: http:// www.herein.at/ (letzter Zugriff: 22.6.2019). 


\section{Autor*innen}

Büro trafo.K ist ein Wiener Büro für Kunstvermittlung und arbeitet seit 1999 an Forschungs- und Vermittlungsprojekten an der Schnittstelle von Bildung und kritischer Wissensproduktion. Dazu gehören Medien- und Jugendprojekte, künstlerische Interventionen, wissenschaftliche Studien, Schulungen, Workshops und Consulting für Museen und Ausstellungen sowie Projekte im öffentlichen Raum. Büro trafo.K sind Renate Höllwart, Elke Smodics und Nora Sternfeld (www.trafo-k.at).

das kollektiv ist ein Ort für kritische Bildungs-, Beratungs- und Kulturarbeit von und für Migrant"innen (www.das-kollektiv.at). Die beiden Verfasserinnen* des Textes Rubia Salgado und Gergana Mineva sind Co-Autorinnen* des Bandes Pädagogik im globalen postkolonialen Raum. Bildungspotenziale von Dekolonisierung und Emanzipation (2019). Beide arbeiten als Erwachsenenbildnerinnen* im Verein das kollektiv und koordinieren Bildungs-, Forschungsund Entwicklungsprojekte. Gergana Mineva ist zudem als Beraterin* im Verein tätig. Rubia Salgado ist Autorin* und Mitbegründerin* von maiz, einem unabhängigen Verein von und für Migrantinnen*.

Renate Höllwart ist Gründungsmitglied von Büro trafo.K und Vermittlerin mit den Schwerpunkten Zeitgeschichte, zeitgenössische Kunst und öffentlicher Raum. Sie lehrt am Institut für das künstlerische Lehramt an der Akademie der bildenden Künste Wien, ist Co-Leiterin des ecm-Masterlehrgangs für Ausstellungstheorie und -praxis an der Universität für angewandte Kunst Wien und im Kernteam von schnittpunkt. ausstellungstheorie \& praxis.

Claudia Hummel ist Kunstvermittlerin und wissenschaftliche Lehrkraft am Institut für Kunst im Kontext an der Universität der Künste Berlin. Schwerpunkte ihrer Arbeit sind Recherchen zum Kontext Schule, zum Handlungs- 
raum Kunstvermittlung und zur Geschichte der künstlerisch-edukativen Praxis in Westdeutschland seit den 1970er Jahren.

Aslı Kışlal ist Regisseurin, Dramaturgin und Drehbuchautorin. Seit 1989 lebt und arbeitet sie als Schauspielerin und Regisseurin in Österreich und Deutschland. 2003 gründete sie das Theaterkollektiv daskunst. 2009 bis 2010 war sie Künstlerische Leiterin des Theaters des Augenblicks, 2011 bis 2012 Initiatorin und Kuratorin der Projektreihe Postmigrantische Positionen PIMP MY INTEGRATION. 2013 gründete sie das Performance- und Theaterlabor diverCITYLAB.

Nora Landkammer ist Kunstvermittlerin und arbeitet in Forschungsprojekten zu Vermittlung mit Schwerpunkt auf Aktions- und partizipative Forschung und auf Rassismus- und Kolonialismuskritik in der Bildungsarbeit und in Kulturinstitutionen. 2008 bis 2018 war sie wissenschaftliche Mitarbeiterin und stellvertretende Leiterin des Institute for Art Education an der Zürcher Hochschule der Künste. Sie lehrt im Bereich Kunstvermittlung in Wien und Zürich. Sie ist aktiv im Kollektiv EAR und im Forschungsnetzwerk Another Roadmap for Arts Education.

Carina Maier studierte Politikwissenschaft und Sozioökonomie in Wien und Bordeaux, war Mitarbeiterin im Projekt Making Democracy und arbeitet als bildungspolitische Trainerin. Ihre Arbeitsschwerpunkte sind feministische Theorien, Antifeminismus, Kritische Gesellschaftstheorie, Feministische Politische Ökonomie, Bildungspolitik und Body Politics.

Oliver Marchart ist Philosoph und seit 2016 Professor für Politische Theorie und Ideengeschichte am Institut für Politikwissenschaft der Universität Wien. Er leitete das Projekt Making Democracy. Seine Arbeitsschwerpunkte sind Demokratietheorie, politische Ideengeschichte, Soziale Bewegungsforschung, politische Diskursanalyse und Populismus.

Ingo Pohn-Lauggas ist Literatur- und Kulturwissenschaftler und leitet die Koordinationsstelle Kulturwissenschaften/Cultural Studies an der Universität Wien. Er ist Redaktionsmitglied von Das Argument - Zeitschrift für Philosophie und Sozialwissenschaften und der Zeitschrift für Kulturwissenschaften sowie Herausgeber der Schriften Antonio Gramscis zur Literatur und Kultur. 
Elke Rajal ist Politikwissenschaftlerin. Sie forscht und lehrt an verschiedenen Hochschulen und Instituten zu den Schnittstellen von Politik, Zeitgeschichte und Bildung. Ihre Arbeitsschwerpunkte sind Nationalsozialismus, Vergangenheitspolitik, Antisemitismus, Geschlecht und Bildung (Politische Bildung, Holocaust Education, Rechtsextremismusprävention, Migrationspädagogik). Sie hat bereits mehrere Forschungs- und Vermittlungsprojekte mit Schüler*innen durchgeführt und engagiert sich in der Forschungsgruppe Ideologien und Politiken der Ungleichheit (FIPU).

Stefanie Schermann studierte Germanistik und Geschichte auf Lehramt sowie Deutsch als Zweit- und Fremdsprache an der Universität Wien. Seit 2015 unterrichtet sie an der WMS/BRG/ORG antonkriegergasse im 23. Wiener Gemeindebezirk.

Karin Schneider ist Kunstvermittlerin und Forschende im Bereich Praxisund Aktionsforschung. Zurzeit hat sie die Leitung der Kunstvermittlung im Lentos Kunstmuseum und im Nordico Stadtmuseum (beide in Linz) inne. 2016 bis 2019 war sie im Rahmen des H2020 Forschungsprojekt TRACES am Institute for Art Education an der Zürcher Hochschule der Künste beschäftigt. Sie ist Co-Autorin des Bandes Partizipative Aktionsforschung mit Kindern und Jugendlichen (2017) und vom Praxishandbuch Sozialwissenschaftliches Forschen mit Kindern und Jugendlichen (2018).

Elke Smodics ist Kommunikationskuratorin mit den Schwerpunkten zeitgenössische Kunst, Feminismus und Partizipation. Ihr Fokus ist die Entwicklung von Vermittlungstools und Arbeitsmaterialien für antidiskriminierende, emanzipatorische Bildungsprozesse. Sie kuratiert partizipative Ausstellungen sowie Projekte im öffentlichen Raum und ist Lehrbeauftragte zu queer-feministischen, transdisziplinären Vermittlungsstrategien. Sie ist Gründungsmitglied von Büro trafo.K.

Nora Sternfeld ist Mitbegründerin und Teilhaberin von Büro trafo.K. Seit Januar 2018 ist sie documenta-Professorin an der Kunsthochschule Kassel. Von 2012 bis 2018 war sie Professorin für Curating and Mediating Art an der Aalto University in Helsinki. Sie ist Co-Leiterin des ecm-Masterlehrgangs für Ausstellungstheorie und -praxis an der Universität für angewandte Kunst Wien, im Kernteam von schnittpunkt. ausstellungstheorie \& praxis und seit 
2011 Teil von freethought, Plattform für Forschung, Bildung und Produktion (London). Sie publiziert zu zeitgenössischer Kunst, Ausstellungen, Geschichtspolitik und Bildungstheorie.

Rahel Süß ist politische Theoretikerin, Autorin von Kollektive Handlungsfähigkeit. Gramsci-Holzkamp - Laclau/Mouffe (2016) und Mitherausgeberin der Zeitschrift engagée - politisch-philosophische Einmischungen. Sie lebt in London, wo sie zu einer radikalen Demokratietheorie des Experiments forscht. Rahel Süß ist Lehrbeauftragte der Universität Wien und derzeit Visiting Scholar am Literature Program und Social Movement Lab der Duke University.

Beate Wallner studierte Germanistik und Geografie und Wirtschaftskunde an der Universität Wien, unterrichtet seit vielen Jahren an der WMS/BRG/ORG antonkriegergasse im 23. Wiener Gemeindebezirk, wo sie auch für Neue Lernkultur zuständig ist.

Elke Zobl leitet den Programmbereich Zeitgenössische Kunst und Kulturproduktion, Schwerpunkt Wissenschaft \& Kunst der Universität Salzburg und Universität Mozarteum (www.w-k.sbg.ac.at) und aktuell das Projekt Kulturelle Teilhabe in Salzburg (www.p-art-icipate.net). 


\section{Politikwissenschaft}

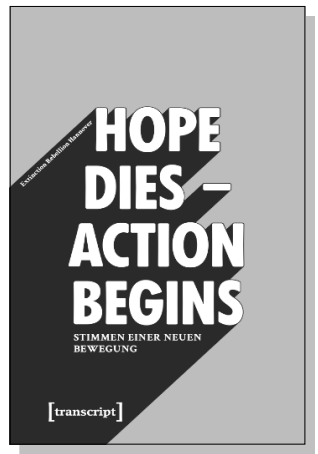

Extinction Rebellion Hannover

"Hope dies - Action begins":

Stimmen einer neuen Bewegung

2019,96 S., kart.

7,99€ (DE), 978-3-8376-5070-9

E-Book: kostenlos erhältlich als Open-Access-Publikation

E-Book: ISBN 978-3-8394-5070-3

EPUB: ISBN 978-3-7328-5070-9

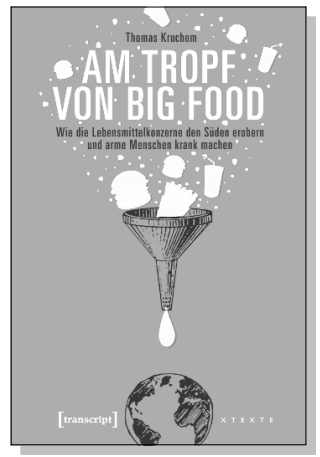

Thomas Kruchem

Am Tropf von Big Food

Wie die Lebensmittelkonzerne den Süden erobern

und arme Menschen krank machen

2017, 214 S., kart., 10 SW-Abbildungen

19,99€ (DE), 978-3-8376-3965-0

E-Book: 16,99 € (DE), ISBN 978-3-8394-3965-4

EPUB: $16,99 €$ (DE), ISBN 978-3-7328-3965-0

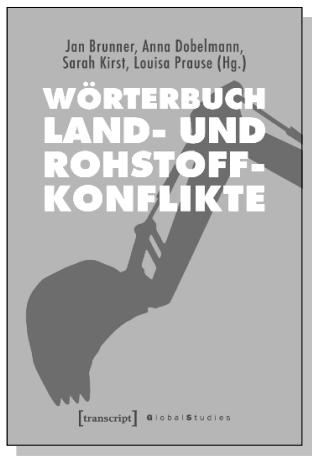

Jan Brunner, Anna Dobelmann, Sarah Kirst, Louisa Prause (Hg.) Wörterbuch Land- und Rohstoffkonflikte

2019, 326 S., kart., Dispersionsbindung, 1 SW-Abbildung 24,99€ (DE), 978-3-8376-4433-3

E-Book: 21,99 € (DE), ISBN 978-3-8394-4433-7 


\section{Politikwissenschaft}

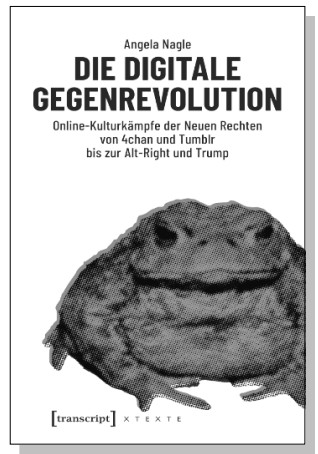

Ines-Jacqueline Werkner Gerechter Frieden Das fortwährende Dilemma militärischer Gewalt

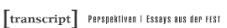

Judith Vey, Johanna Leinius, Ingmar Hagemann $(\mathrm{Hg}$.

\section{Handbuch}

Poststrukturalistische

Perspektiven auf

soziale Bewegungen

Ansätze, Methoden

und Forschungspraxis

\section{Angela Nagle}

Die digitale Gegenrevolution

Online-Kulturkämpfe der Neuen Rechten

von 4chan und Tumblr bis zur Alt-Right und Trump

2018, 148 S., kart.

$19,99 €(D E), 978-3-8376-4397-8$

E-Book: 17,99 € (DE), ISBN 978-3-8394-4397-2

EPUB: $17,99 €$ (DE), ISBN 978-3-7328-4397-8

Ines-Jacqueline Werkner

\section{Gerechter Frieden}

Das fortwährende Dilemma militärischer Gewalt

2018, 106 S., kart.

$14,99 €(D E), 978-3-8376-4074-8$

E-Book: 12,99 € (DE), ISBN 978-3-8394-4074-2

Judith Vey, Johanna Leinius, Ingmar Hagemann (Hg.)

\section{Handbuch Poststrukturalistische Perspektiven} auf soziale Bewegungen

Ansätze, Methoden und Forschungspraxis

2019, 306 S., kart., Dispersionsbindung,

2 SW-Abbildungen, 2 Farbabbildungen

24,99€ (DE), 978-3-8376-4879-9

E-Book: kostenlos erhältlich als Open-Access-Publikation

E-Book: ISBN 978-3-8394-4879-3 